\title{
Alkylation of Nitropyridines via Vicarious Nucleophilic Substitution
}

Damian Antoniak ${ }^{1}$ and Michał Barbasiewicz ${ }^{1, *}$

${ }^{1}$ Faculty of Chemistry, University of Warsaw

Pasteura 1, 02-093 Warsaw, Poland

*barbasiewicz@chem.uw.edu.pl

www.aromaticity.pl 


\section{Table of content}

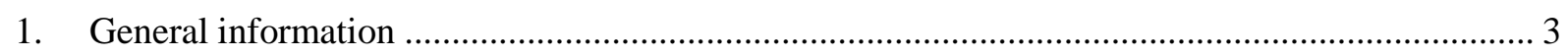

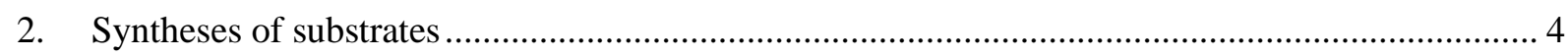

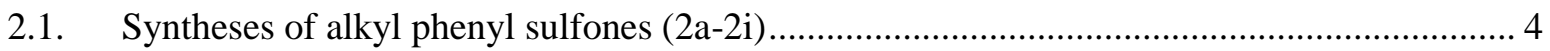

2.2. Syntheses of neopentyl alkanesulfonates (3a-3c, 3e) ..................................................... 15

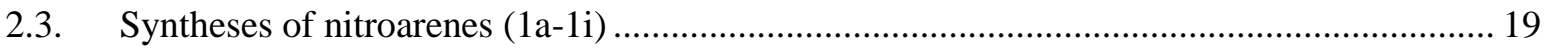

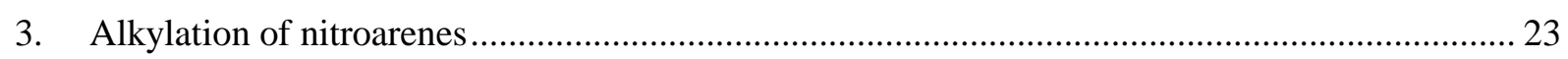

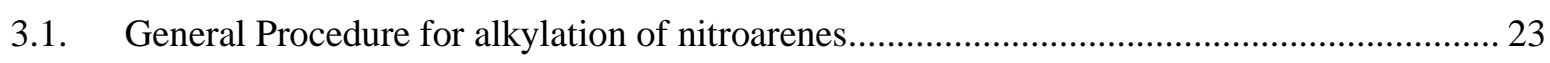

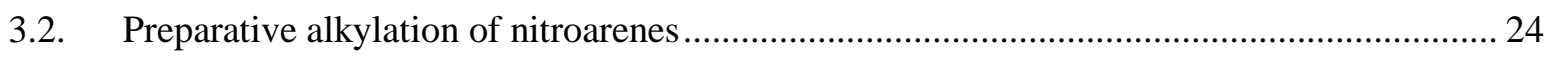

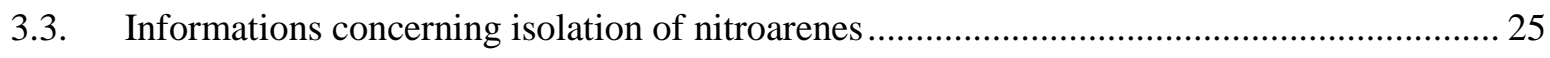

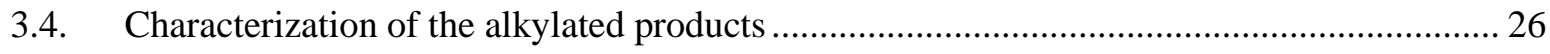

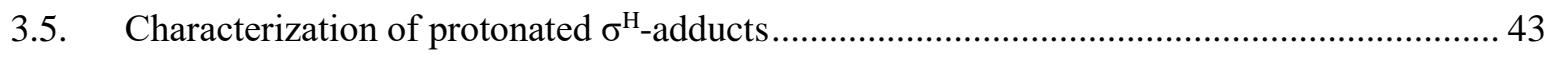

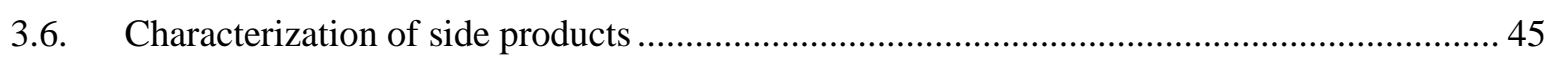

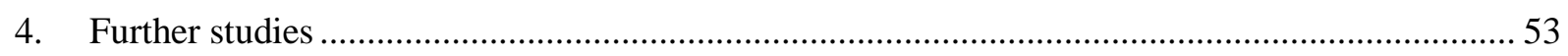

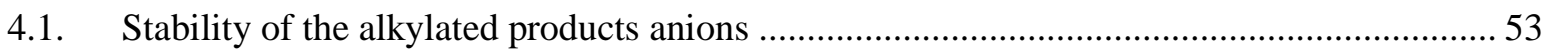

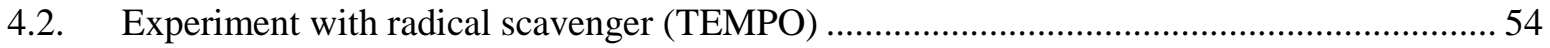

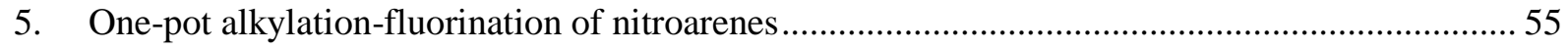

6. Postsynthetic transformations of the alkylated pyridines ............................................................. 58

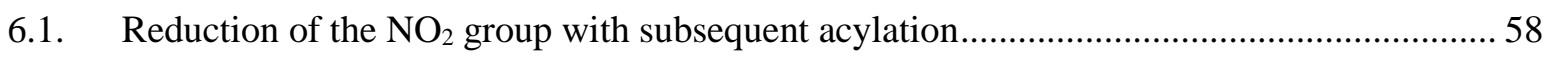

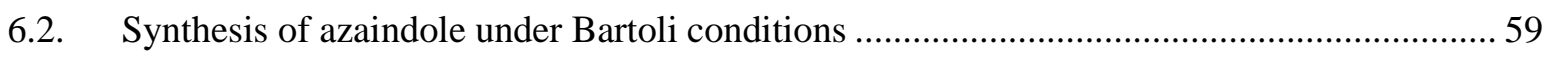

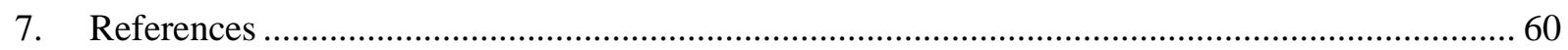

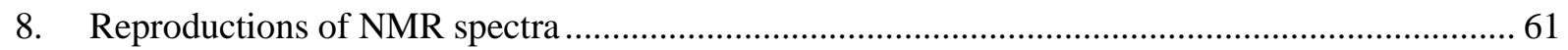




\section{General information}

Methyl iodide, isopropyl bromide, and 4-chloronitrobenzene were purchased from Acros Organics.

Diethyl azodicarboxylate and tert-butyl hydroperoxide $\left(70 \%\right.$ solution in $\left.\mathrm{H}_{2} \mathrm{O}\right)$ were purchased from Alfa Aesar.

1-Methyl-4-piperidinemethanol, 2-chloro-5-nitropyridine, 4-methoxy-3-nitropyridine, and TEMPO were purchased from AmBeed.

$\mathrm{K}_{2} \mathrm{CO}_{3}, \mathrm{KOH}, \mathrm{Na}_{2} \mathrm{CO}_{3}, \mathrm{Na}_{2} \mathrm{SO}_{3}, \mathrm{MgSO}_{4}, \mathrm{NH}_{4} \mathrm{Cl}$, acetic acid, and acetic anhydride were purchased from Chempur, Poland.

Methanesulfonyl chloride and $\mathrm{Pd} / \mathrm{C}$ were purchased from Fluka.

LiCl, cyclopropylmethyl bromide, octanesulfonyl chloride, and 2-nitrothiophene were purchased from Fluorochem.

Triethylamine, isobutyl iodide, $\mathrm{NaCl}$, and $\mathrm{NaOH}$ were purchased from $\underline{\mathrm{POCh}}$, Poland.

Thiophenol, neopentyl alcohol, ethyl bromide, ethanesulfonyl chloride, isobutanesulfonyl chloride, 3 -aminoquinoline, tetrabutylammonium chloride, benzyltriethylammonium chloride, $\mathrm{NaH}$ (60\% in oil), sodium benzenesulfinate, 4-bromobut-1-ene, octyl bromide, triphenylphosphine, 3-nitropyridine, 2,4,6-trichlorophenol, 2-bromo-5-nitropyridine, vinylmagnesium bromide (1.0 $\mathrm{M}$ in $\mathrm{THF}$ ), $\mathrm{CHCl}_{3}$, $\mathrm{MeOH}$, dry DMF, dry THF and dry DMSO were purchased from Sigma-Aldrich.

KHMDS (solution in THF) was purchased from Sigma-Aldrich and Fluorochem (1 M from SigmaAldrich and 20\% w/w from Fluorochem).

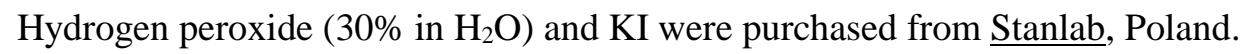

Selectfluor was purchased from Apollo.

Commercially available solvents and materials were used without further purification.

Column chromatography was performed on silica gel (high-purity grade, pore size $60 \AA, 230-400$ mesh particle size, $40-63 \mu \mathrm{m})$.

Thin layer chromatography (TLC) was performed on Supelco Silica gel $60 \mathrm{~F}_{254}$ aluminium sheets and was visualized under UV lamp. In some cases, solution of $\mathrm{KMnO}_{4}$ was used for visualization (prepared form $0.6 \mathrm{~g} \mathrm{KMnO}_{4}, 4 \mathrm{~g} \mathrm{~K}_{2} \mathrm{CO}_{3}, 5 \mathrm{mg} \mathrm{NaOH}$ and $80 \mathrm{~mL} \mathrm{H}_{2} \mathrm{O}$ ).

Analytical GLC was performed on a PerkinElmer Clarus 580 chromatograph equipped with a flame ionization detector, and a GL Sciences InertCap 5MS/Sil column with $\mathrm{He}$ as a carrier gas (column $0.25 \mathrm{~mm} \times 30 \mathrm{~m}$, carrier flow $1.5 \mathrm{~mL} / \mathrm{min}$, method parameters $50{ }^{\circ} \mathrm{C},+10^{\circ} \mathrm{C} / \mathrm{min}$ to $300{ }^{\circ} \mathrm{C}$, then $15 \mathrm{~min}$ at $\left.300^{\circ} \mathrm{C}\right)$.

${ }^{1} \mathrm{H},{ }^{19} \mathrm{~F}$, and ${ }^{13} \mathrm{C}$ NMR spectra were recorded on Agilent $400 \mathrm{MHz}$ NMR spectrometer. Chemical shifts $(\delta)$ are given in parts per million (ppm) with solvent resonance as internal standard (for $\mathrm{CDCl}_{3}: 7.24$ and $77.0 \mathrm{ppm}$, for ${ }^{1} \mathrm{H}$ and ${ }^{13} \mathrm{C}$ NMR, respectively; for DMSO- $d_{6}: 2.50$ and $40.0 \mathrm{ppm}$, for ${ }^{1} \mathrm{H}$ and ${ }^{13} \mathrm{C}$ NMR, respectively) or with $\mathrm{CFCl}_{3}\left(0.0 \mathrm{ppm}\right.$ for $\left.{ }^{19} \mathrm{~F} \mathrm{NMR}\right)$. Spin multiplicity was abbreviated as follows: $\mathrm{s}$, singlet; d, doublet; t, triplet; q, quartet; hept, heptet; m, multiplet; br s, broad singlet.

Melting points were uncorrected. 


\section{Syntheses of substrates}

\subsection{Syntheses of alkyl phenyl sulfones (2a-2i)}

Syntheses of alkyl phenyl sulfones (2a-2i) were described below:<smiles>CS(=O)(=O)c1ccccc1</smiles>

$2 \mathrm{a}$<smiles>O=S(=O)(CC1CC1)c1ccccc1</smiles>

$2 f$<smiles>CCS(=O)(=O)c1ccccc1</smiles>

$2 b$<smiles>CC(C)CS(=O)(=O)c1ccccc1</smiles>

2c<smiles>CC(C)(C)CS(=O)(=O)c1ccccc1</smiles>

$2 d$<smiles>CCCCCCCCS(=O)(=O)c1ccccc1</smiles>

$2 e$<smiles>O=S(=O)(CCC1CC1(Cl)Cl)c1ccccc1</smiles>

$2 \mathrm{~g}$<smiles>CN1CCC(CS(=O)(=O)c2ccccc2)CC1</smiles>

$2 \mathrm{~h}$<smiles>CC(C)S(=O)(=O)c1ccccc1</smiles>

$\mathbf{2 i}$ 


\section{Synthesis of methyl phenyl sulfone (2a)}

Methyl phenyl sulfone was prepared according to the reported procedure. ${ }^{[1]}$

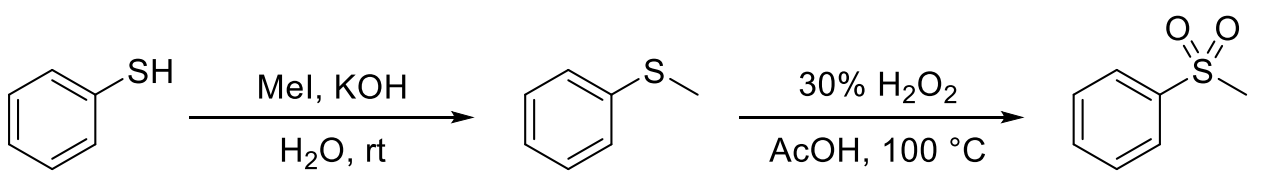

2a, $82 \%$

over 2 steps

A $100 \mathrm{~mL}$ round-bottom flask was charged with thiophenol $(5.50 \mathrm{~g}, 49.9 \mathrm{mmol}, 1.00$ equiv.) and immersed in water bath (rt). To the stirred reaction mixture solution of $\mathrm{KOH}(4.22 \mathrm{~g}, 75.2 \mathrm{mmol}, 1.51$ equiv.) in $\mathrm{H}_{2} \mathrm{O}(35 \mathrm{~mL}$ ) was added portionwise, then methyl iodide ( $7.75 \mathrm{~g}, 54.6 \mathrm{mmol}, 1.09$ equiv.) was added dropwise. After $2 \mathrm{~h}$ reaction mixture was extracted with ethyl acetate $(3 \times 50 \mathrm{~mL})$, combined organic phases were washed with $\mathrm{H}_{2} \mathrm{O}(50 \mathrm{~mL})$, brine $(50 \mathrm{~mL})$, and dried over anhydrous $\mathrm{MgSO}_{4}$. The mixture was filtered and evaporated. Crude methyl phenyl sulfide was placed in $100 \mathrm{~mL}$ round-bottom flask, acetic acid $(20 \mathrm{~mL})$ was added, to the stirred reaction mixture $\mathrm{H}_{2} \mathrm{O}_{2}$ aq $(22 \mathrm{~mL}, 216 \mathrm{mmol}, 30 \%$ $\mathrm{v} / \mathrm{v}, 4.33$ equiv.) was added dropwise, and reaction was heated to ca. $100^{\circ} \mathrm{C}$ (oil bath). After $3 \mathrm{~h}$ reaction mixture was poured onto water/ice $(300 \mathrm{~mL})$, solid $\mathrm{Na}_{2} \mathrm{CO}_{3}$ was added to $\mathrm{pH}=7$, mixture was extracted with ethyl acetate $(3 \times 50 \mathrm{~mL})$, combined organic phases were washed with $\mathrm{Na}_{2} \mathrm{SO}_{3}$ aq $(50 \mathrm{~mL}, 10 \%$ $\mathrm{w} / \mathrm{v})$, brine $(50 \mathrm{~mL})$, and dried over anhydrous $\mathrm{MgSO}_{4}$. Mixture was filtered, evaporated, and crude product was recrystallized form ethanol yielding $\mathbf{2 a}(6.63 \mathrm{~g}, 41.1 \mathrm{mmol}, \mathbf{8 2} \%$ over 2 steps) as white crystals.<smiles>CS(=O)(=O)c1ccccc1</smiles>

methyl phenyl sulfone, $\mathbf{2 a}$
white crystals, $\mathbf{m p} 86.5-88.0{ }^{\circ} \mathrm{C}$

${ }^{1} \mathbf{H}$ NMR (400 MHz, $\left.\mathbf{C D C l}_{3}\right): \delta=7.95-7.91(\mathrm{~m}, 2 \mathrm{H}), 7.67-7.62(\mathrm{~m}$, $1 \mathrm{H}), 7.58-7.53(\mathrm{~m}, 2 \mathrm{H}), 3.04(\mathrm{~s}, 3 \mathrm{H})$.

${ }^{13}$ C NMR (100 MHz, CDCl $)$ ): $\delta=140.2,133.4,129.1,126.9,44.1$.

${ }^{1} \mathrm{H}$ NMR, ${ }^{13} \mathrm{C}$ NMR spectra and mp were consistent with those reported in the literature. ${ }^{[2]}$ 


\section{Synthesis of ethyl phenyl sulfone (2b)}

Ethyl phenyl sulfone was prepared according to the reported procedure. ${ }^{[1]}$

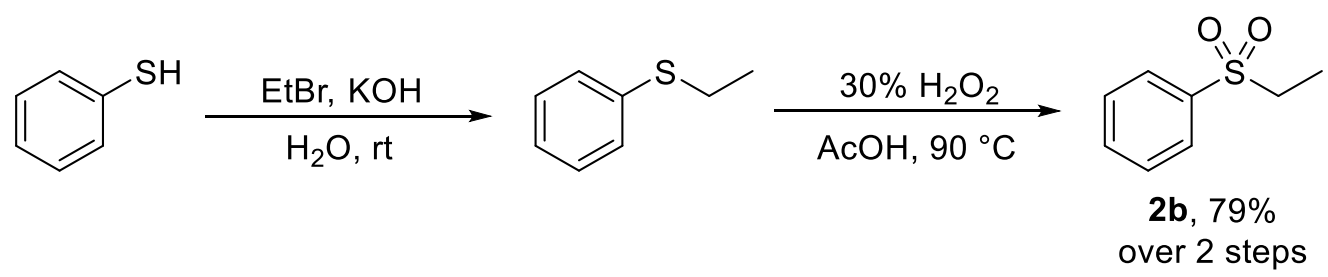

A $100 \mathrm{~mL}$ round-bottom flask was charged with thiophenol $(5.50 \mathrm{~g}, 49.9 \mathrm{mmol}, 1.00$ equiv.) and immersed in water bath (rt). To the stirred reaction mixture solution of $\mathrm{KOH}(4.21 \mathrm{~g}, 75.1 \mathrm{mmol}, 1.51$ equiv.) in $\mathrm{H}_{2} \mathrm{O}(35 \mathrm{~mL}$ ) was added portionwise, and then ethyl bromide ( $6.00 \mathrm{~g}, 54.9 \mathrm{mmol}, 1.10$ equiv.) was added dropwise. After $2 \mathrm{~h}$ mixture was extracted with ethyl acetate $(3 \times 50 \mathrm{~mL})$, combined organic phases were washed with $\mathrm{H}_{2} \mathrm{O}(50 \mathrm{~mL})$, brine $(50 \mathrm{~mL})$, and dried over anhydrous $\mathrm{MgSO}_{4}$. The mixture was filtered and evaporated. Crude ethyl phenyl sulfide was placed in $100 \mathrm{~mL}$ round-bottom flask, acetic acid $(20 \mathrm{~mL})$ was added, to the stirred reaction mixture $\mathrm{H}_{2} \mathrm{O}_{2}$ aq $(16 \mathrm{~mL}, 157 \mathrm{mmol}, 30 \% \mathrm{v} / \mathrm{v}, 3.15$ equiv.) was added dropwise, and reaction mixture was heated to ca. $90{ }^{\circ} \mathrm{C}$ (oil bath). After $3 \mathrm{~h}$ reaction mixture was poured onto water/ice $(300 \mathrm{~mL})$, solid $\mathrm{Na}_{2} \mathrm{CO}_{3}$ was added to $\mathrm{pH}=7$, mixture was extracted with ethyl acetate $(3 \times 50 \mathrm{~mL})$, combined organic phases were washed with $\mathrm{Na}_{2} \mathrm{SO}_{3}$ aq $(50 \mathrm{~mL}, 10 \% \mathrm{w} / \mathrm{v})$, brine $(50 \mathrm{~mL})$, and dried over anhydrous $\mathrm{MgSO}_{4}$. Mixture was filtered, evaporated, and crude product was recrystallized from ethanol yielding $\mathbf{2 b}(6.71 \mathrm{~g}, 39.4 \mathrm{mmol}, \mathbf{7 9 \%}$ over 2 steps $)$ as white crystals.<smiles>CCS(=O)(=O)c1ccccc1</smiles>

ethyl phenyl sulfone, $\mathbf{2 b}$

white crystals, $\mathbf{m p} 41.0-42.5^{\circ} \mathrm{C}$

${ }^{1} \mathbf{H}$ NMR $\left(400 \mathrm{MHz}, \mathbf{C D C l}_{3}\right): \delta=7.92-7.86(\mathrm{~m}, 2 \mathrm{H}), 7.67-7.60(\mathrm{~m}$, $1 \mathrm{H}), 7.58-7.46(\mathrm{~m}, 2 \mathrm{H}), 3.09(\mathrm{q}, J=7.4 \mathrm{~Hz}, 2 \mathrm{H}), 1.25(\mathrm{t}, J=7.4 \mathrm{~Hz}, 3 \mathrm{H})$.

${ }^{13}$ C NMR (100 MHz, CDCl $)$ : $\delta=138.3,133.5,129.1,128.0,50.4,7.3$.

${ }^{1} \mathrm{H}$ NMR, ${ }^{13} \mathrm{C}$ NMR spectra were consistent with those reported in the literature. ${ }^{[1]}$ 


\section{Synthesis of isobutyl phenyl sulfone (2c)}

Isobutyl phenyl sulfone was prepared according to the reported procedure ${ }^{[1]}$ modified by addition of phase transfer catalyst at the alkylation step.

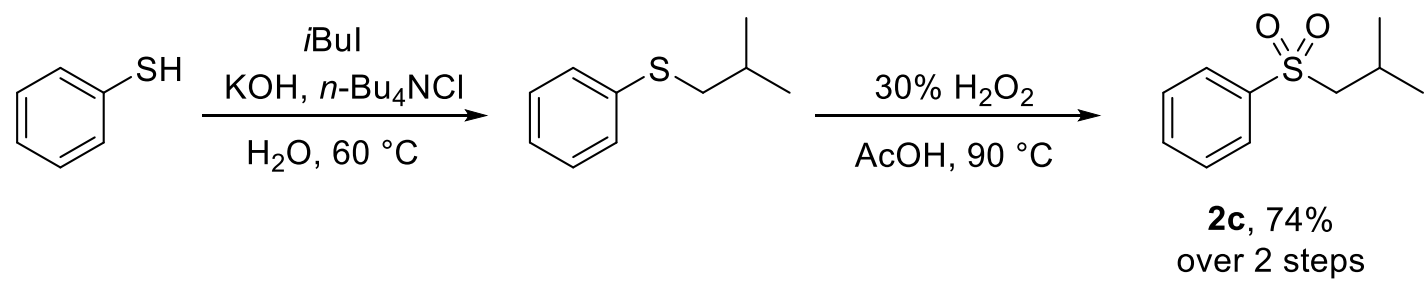

A $250 \mathrm{~mL}$ round-bottom flask was charged with thiophenol (11.3 g, $103 \mathrm{mmol}, 1.00$ equiv.) and immersed in water bath (rt). To vigorously stirred reaction mixture solution of $\mathrm{KOH}(8.63 \mathrm{~g}, 154 \mathrm{mmol}$, 1.50 equiv.) in $\mathrm{H}_{2} \mathrm{O}(70 \mathrm{~mL}$ ) was added portionwise, then isobutyl iodide (27.2 g, $148 \mathrm{mmol}, 1.44$ equiv.) was added dropwise. Tetrabutylammonium chloride $(0.28 \mathrm{~g}, 1.01 \mathrm{mmol}, 1.0 \mathrm{~mol} \%)$ was added, and reaction mixture was heated to $60^{\circ} \mathrm{C}$ (oil bath). After $16 \mathrm{~h}$ reaction mixture was extracted with ethyl acetate $(3 \times 50 \mathrm{~mL})$, combined organic phases were washed with $\mathrm{H}_{2} \mathrm{O}(50 \mathrm{~mL})$, brine $(50 \mathrm{~mL})$, and dried over anhydrous $\mathrm{MgSO}_{4}$. The mixture was filtered and evaporated. Crude isobutyl phenyl sulfide was placed in $250 \mathrm{~mL}$ round-bottom flask, acetic acid $(40 \mathrm{~mL})$ was added, to the stirred reaction mixture $\mathrm{H}_{2} \mathrm{O}_{2}$ aq $(32 \mathrm{~mL}, 314 \mathrm{mmol}, 30 \% \mathrm{v} / \mathrm{v}, 3.05$ equiv.) was added dropwise, and reaction mixture was heated to ca. $90{ }^{\circ} \mathrm{C}$ (oil bath). After $2.5 \mathrm{~h}$ reaction mixture was poured onto water/ice $(400 \mathrm{~mL})$, solid $\mathrm{Na}_{2} \mathrm{CO}_{3}$ was added to $\mathrm{pH}=7$, mixture was extracted with ethyl acetate $(3 \times 100 \mathrm{~mL})$, combined organic phases were washed with $\mathrm{Na}_{2} \mathrm{SO}_{3}$ aq $\left(100 \mathrm{~mL}, 10 \%\right.$ w/v), brine $(100 \mathrm{~mL})$, and dried over anhydrous $\mathrm{MgSO}_{4}$. The mixture was filtered, evaporated, and product was separated by distillation $\left(114-118{ }^{\circ} \mathrm{C}, 4 \times 10^{-2}\right.$ mbar) yielding $2 \mathrm{c}$ (15.1 g, $76.0 \mathrm{mmol}, \mathbf{7 4 \%}$ over 2 steps) as a light orange oil.<smiles>CC(C)CS(=O)(=O)c1ccccc1</smiles>

isobutyl phenyl sulfone, $2 \mathrm{c}$

light orange oil

${ }^{1} \mathbf{H}$ NMR (400 MHz, $\left.\mathbf{C D C l}_{3}\right): \delta=7.91-7.81(\mathrm{~m}, 2 \mathrm{H}), 7.64-7.58(\mathrm{~m}$, $1 \mathrm{H}), 7.56-7.48(\mathrm{~m}, 2 \mathrm{H}), 2.95(\mathrm{~d}, J=6.4 \mathrm{~Hz}, 2 \mathrm{H}), 2.26-2.11(\mathrm{~m}, 1 \mathrm{H})$, $1.01(\mathrm{~d}, J=6.8 \mathrm{~Hz}, 6 \mathrm{H})$.

${ }^{13} \mathbf{C}$ NMR (100 MHz, $\left.\mathbf{C D C l}_{3}\right): \delta=140.0,133.5,129.2,127.7,63.8,24.0$, 22.6.

${ }^{1} \mathrm{H}$ NMR, ${ }^{13} \mathrm{C}$ NMR spectra were consistent with those reported in the literature. ${ }^{[3]}$ 


\section{Synthesis of neopentyl phenyl sulfone (2d)}

Neopentyl phenyl sulfone was prepared according to modified procedure reported in the literature. ${ }^{[4]}$<smiles>Sc1ccccc1</smiles>

1. $\mathrm{NaH}(60 \%$ in oil) DMF, $2-3{ }^{\circ} \mathrm{C}$ to $\mathrm{rt}$

2. $\mathrm{MeSO}_{2} \mathrm{OCH}_{2} \mathrm{tBu}$ (3a)

DMF, $80{ }^{\circ} \mathrm{C}$<smiles>CC(C)(C)CSc1ccccc1</smiles><smiles>CC(C)(C)CS(=O)(=O)c1ccccc1</smiles>

2d, $90 \%$

over 2 steps

A $100 \mathrm{~mL}$ round-bottom flask was charged with thiophenol ( $3.71 \mathrm{~g}, 33.6 \mathrm{mmol}, 1.12$ equiv.) and flushed with argon. Thiophenol was dissolved in dry DMF $(20 \mathrm{~mL})$, and flask was immersed in ice/water bath $\left(2-3{ }^{\circ} \mathrm{C}\right)$. To the stirred reaction mixture $\mathrm{NaH}(1.37 \mathrm{~g}, 34.3 \mathrm{mmol}, 60 \%$ in mineral oil, 1.14 equiv.) was added portionwise. After 15 min cooling bath was removed and mixture was stirred for $45 \mathrm{~min}$ at $\mathrm{rt}$. Then solution of neopentyl methanesulfonate (3a) $(4.99 \mathrm{~g}, 30.0 \mathrm{mmol}, 1.00$ equiv.) in dry DMF $(10 \mathrm{~mL})$ was added, and reaction mixture was stirred at $80{ }^{\circ} \mathrm{C}$ (oil bath). After $20 \mathrm{~h}$ mixture was transferred to separatory funnel, $\mathrm{NH}_{4} \mathrm{Cl}_{\text {aq }}(50 \mathrm{~mL}, 10 \% \mathrm{w} / \mathrm{v})$ was added, mixture was extracted with ethyl acetate (3 $\times 50 \mathrm{~mL})$, combined organic phases were washed with $\mathrm{LiCl}_{\text {aq }}(50 \mathrm{~mL}, 1.0 \mathrm{M}), \mathrm{Na}_{2} \mathrm{CO}_{3}$ aq $(50 \mathrm{~mL}, 10 \%$ $\mathrm{w} / \mathrm{v})$, brine $(50 \mathrm{~mL})$, and dried over anhydrous $\mathrm{MgSO}_{4}$. The mixture was filtered and evaporated. Crude neopentyl phenyl sulfide was placed in $100 \mathrm{~mL}$ round-bottom flask, acetic acid (12 mL) was added, to the stirred reaction mixture $\mathrm{H}_{2} \mathrm{O}_{2}$ aq $(7.0 \mathrm{~mL}, 68.6 \mathrm{mmol}, 30 \% \mathrm{v} / \mathrm{v}, 2.29$ equiv.) was added, and reaction mixture was heated to $90^{\circ} \mathrm{C}$ (oil bath). After $2 \mathrm{~h}$ reaction mixture was poured onto water/ice $(200 \mathrm{~mL})$, solid $\mathrm{Na}_{2} \mathrm{CO}_{3}(11.3 \mathrm{~g})$ was added, mixture was extracted with ethyl acetate $(3 \times 100 \mathrm{~mL})$, combined organic phases were washed with $\mathrm{Na}_{2} \mathrm{SO}_{3}$ aq $(100 \mathrm{~mL}, 10 \% \mathrm{w} / \mathrm{v})$, brine $(100 \mathrm{~mL})$, and dried over anhydrous $\mathrm{MgSO}_{4}$. The mixture was filtered, evaporated, and separated with column chromatography (eluent: cyclohexane/ethyl acetate 20:1 to 5:1) yielding $\mathbf{2 d}(5.73 \mathrm{~g}, 27.0 \mathrm{mmol}, \mathbf{9 0 \%}$ over 2 steps) as a white solid.<smiles>CC(C)(C)CS(=O)(=O)c1ccccc1</smiles>

neopentyl phenyl sulfone, $\mathbf{2 d}$

white solid, mp $39.0-40.0^{\circ} \mathrm{C}$

eluent: cyclohexane/ethyl acetate 20:1 to 5:1

${ }^{1}$ H NMR (400 MHz, $\left.\mathbf{C D C l}_{3}\right): \delta=7.89-7.83(\mathrm{~m}, 2 \mathrm{H}), 7.60-7.54(\mathrm{~m}$, $1 \mathrm{H}), 7.53-7.46(\mathrm{~m}, 2 \mathrm{H}), 3.00(\mathrm{~s}, 2 \mathrm{H}), 1.14(\mathrm{~s}, 9 \mathrm{H})$.

${ }^{13} \mathbf{C}$ NMR (100 MHz, $\left.\mathbf{C D C l}_{3}\right): \delta=141.7,133.2,129.1,127.4,67.5,32.4$, 29.7.

${ }^{1} \mathrm{H}$ NMR spectrum ${ }^{[5]}$ and $\mathrm{mp}^{[6]}$ were consistent with those reported in the literature. 


\section{Synthesis of octyl phenyl sulfone (2e)}

Octyl phenyl sulfone was prepared according to the reported procedure, ${ }^{[1]}$ modified by addition of phase transfer catalyst at the alkylation step.

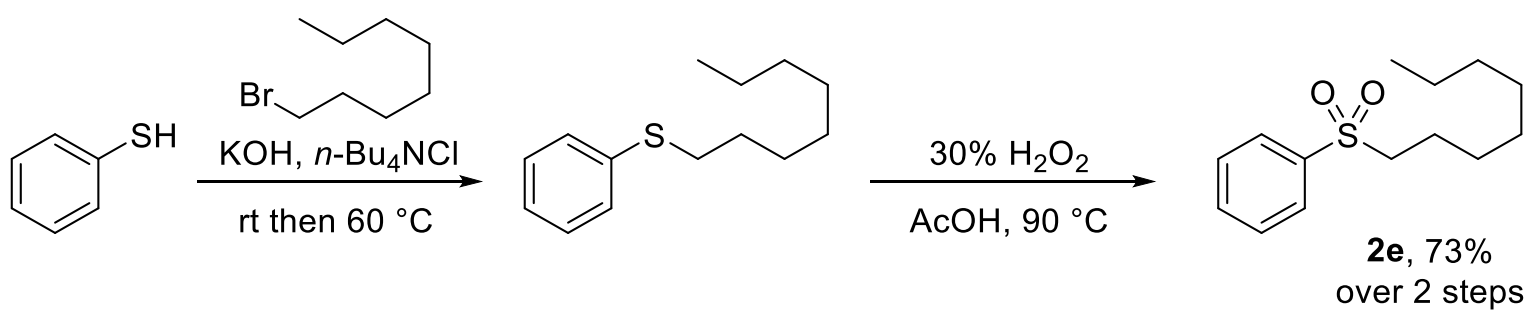

A $100 \mathrm{~mL}$ round-bottom flask was charged with thiophenol $(5.75 \mathrm{~g}, 52.2 \mathrm{mmol}, 1.00$ equiv.) and immersed in water bath (rt). To the stirred reaction mixture solution of $\mathrm{KOH}(4.35 \mathrm{~g}, 78.3 \mathrm{mmol}, 1.50$ equiv.) in $\mathrm{H}_{2} \mathrm{O}(36 \mathrm{~mL})$ was added portionwise, then octyl bromide (11.2 g, $57.9 \mathrm{mmol}, 1.11$ equiv.) was added dropwise. Tetrabutylammonium chloride $(83.0 \mathrm{mg}, 1.01 \mathrm{mmol}, 1.0 \mathrm{~mol} \%)$ was added, and reaction mixture was vigorously stirred for $19 \mathrm{~h}$ at $\mathrm{rt}$, and for $4 \mathrm{~h}$ at $60{ }^{\circ} \mathrm{C}$ (oil bath). Then reaction mixture was extracted with ethyl acetate $(3 \times 50 \mathrm{~mL})$, combined organic phases were washed with $\mathrm{H}_{2} \mathrm{O}$ $(50 \mathrm{~mL})$, brine $(50 \mathrm{~mL})$, and dried over anhydrous $\mathrm{MgSO}_{4}$. The mixture was filtered and evaporated. Crude octyl phenyl sulfide was placed in $100 \mathrm{~mL}$ round-bottom flask, acetic acid $(20 \mathrm{~mL})$ was added, to the stirred reaction mixture $\mathrm{H}_{2} \mathrm{O}_{2}$ aq $(15 \mathrm{~mL}, 147 \mathrm{mmol}, 30 \% \mathrm{v} / \mathrm{v}, 2.82$ equiv.) was added dropwise, and reaction mixture was heated to ca. $90{ }^{\circ} \mathrm{C}$ (oil bath). After $2 \mathrm{~h}$ reaction mixture was poured onto water/ice $(200 \mathrm{~mL})$, solid $\mathrm{Na}_{2} \mathrm{CO}_{3}$ was added to $\mathrm{pH}=7$, mixture was extracted with ethyl acetate $(3 \times$ $50 \mathrm{~mL})$, combined organic phases were washed with $\mathrm{Na}_{2} \mathrm{SO}_{3}$ aq $(50 \mathrm{~mL}, 10 \% \mathrm{w} / \mathrm{v})$, brine $(50 \mathrm{~mL})$, and dried over anhydrous $\mathrm{MgSO}_{4}$. The mixture was filtered, evaporated, and product was dried under high vacuum yielding $2 \mathbf{e}$ ( $9.69 \mathrm{~g}, 38.1 \mathrm{mmol}$, 73\% over 2 steps) as a colorless oil.

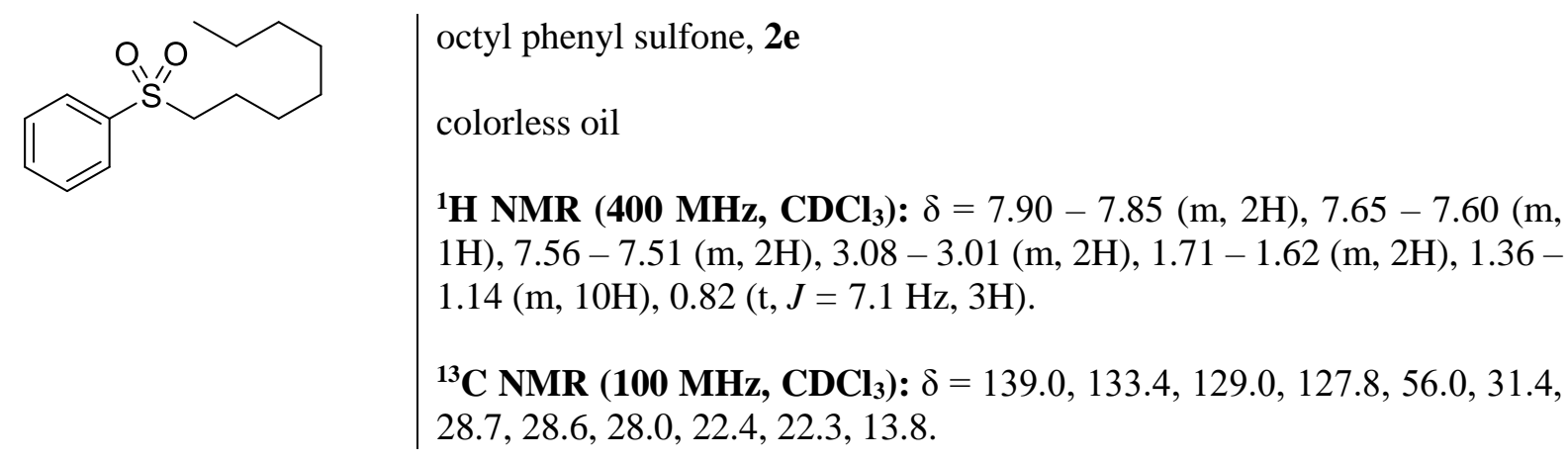

${ }^{1} \mathrm{H}$ NMR, ${ }^{13} \mathrm{C}$ NMR spectra were consistent with those reported in the literature. ${ }^{[7]}$ 


\section{Synthesis of cyclopropylmethyl phenyl sulfone (2f)}

Cyclopropylmethyl phenyl sulfone was prepared from sodium benzenesulfinate and cyclopropylmethyl bromide in DMF at $\mathrm{rt}$.

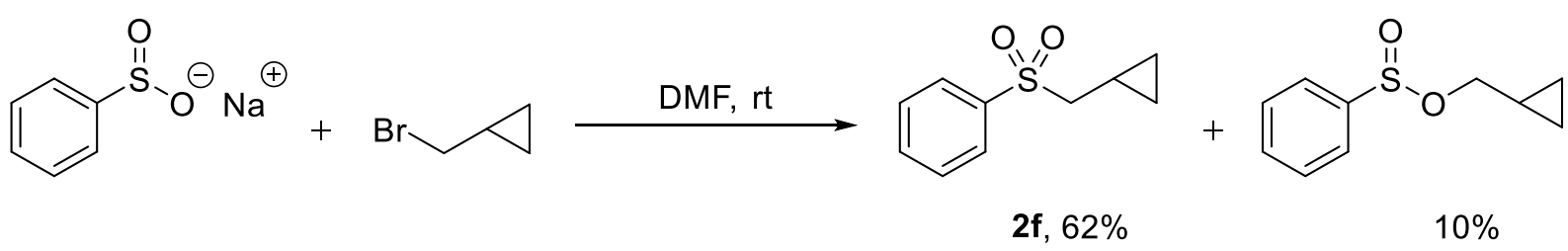

A $50 \mathrm{~mL}$ round-bottom flask was charged with sodium benzenesulfinate $(4.11 \mathrm{~g}, 25.0 \mathrm{mmol}, 1.00$ equiv.), DMF (15 mL), and immersed in water bath (rt). To the stirred reaction mixture cyclopropylmethyl bromide ( $4.04 \mathrm{~g}, 29.9$ mmol, 1.20 equiv.) was added dropwise. After $22 \mathrm{~h} \mathrm{H}_{2} \mathrm{O}$ (50 $\mathrm{mL}$ ) was added, mixture was extracted with ethyl acetate $(3 \times 50 \mathrm{~mL})$, combined organic phases were washed with $\mathrm{LiCl}_{\mathrm{aq}}(50 \mathrm{~mL}, 1.0 \mathrm{M}), \mathrm{H}_{2} \mathrm{O}(50 \mathrm{~mL})$, brine $(50 \mathrm{~mL})$, and dried over anhydrous $\mathrm{MgSO}_{4}$. The mixture was filtered, evaporated and separated with column chromatography (eluent: cyclohexane/ethyl acetate 10:1 to 3:1) yielding $2 \mathbf{f}$ (3.06 $\mathrm{g}, 15.6 \mathrm{mmol}, \mathbf{6 2 \%} \%$, more polar fraction) as a colorless oil, and cyclopropylmethyl benzenesulfinate $(0.483 \mathrm{~g}, 2.46 \mathrm{mmol}, \mathbf{1 0} \%$, less polar fraction) as a pale yellow oil.

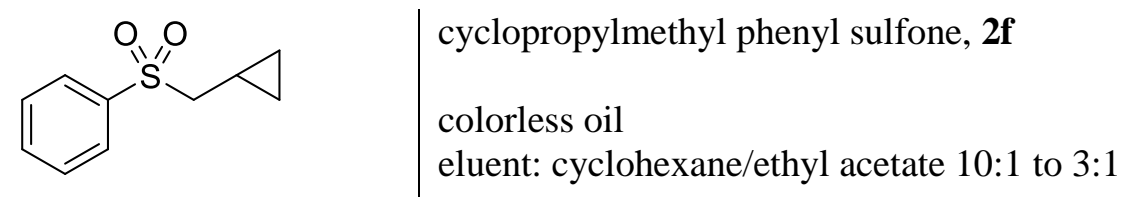

${ }^{1}$ H NMR (400 MHz, CDCl $): \delta=7.83-7.78(\mathrm{~m}, 2 \mathrm{H}), 7.56-7.51(\mathrm{~m}$, $1 \mathrm{H}), 7.47-7.41(\mathrm{~m}, 2 \mathrm{H}), 2.91(\mathrm{~d}, J=7.2 \mathrm{~Hz}, 2 \mathrm{H}), 0.92-0.79(\mathrm{~m}, 1 \mathrm{H})$, $0.45-0.37$ (m, 2H), $0.04--0.04(\mathrm{~m}, 2 \mathrm{H})$.

${ }^{13}$ C NMR (100 MHz, $\left.\mathbf{C D C l}_{3}\right): \delta=138.9,133.4,128.9,128.1,60.9,4.5$, 4.1.

${ }^{1} \mathrm{H}$ NMR, ${ }^{13} \mathrm{C}$ NMR spectra were consistent with those reported in the literature. ${ }^{[8]}$<smiles>O=S(OCC1CC1)c1ccccc1</smiles>

cyclopropylmethyl benzenesulfinate

pale yellow oil

eluent: cyclohexane/ethyl acetate 10:1 to $3: 1$

${ }^{1} \mathbf{H}$ NMR (400 MHz, $\left.\mathbf{C D C l}_{3}\right): \delta=7.66-7.60(\mathrm{~m}, 2 \mathrm{H}), 7.48-7.41(\mathrm{~m}$, $3 \mathrm{H}), 3.76(\mathrm{dd}, J=10.7,7.4 \mathrm{~Hz}, 1 \mathrm{H}), 3.41(\mathrm{dd}, J=10.7,7.1 \mathrm{~Hz}, 1 \mathrm{H}), 1.06$ $-0.95(\mathrm{~m}, 1 \mathrm{H}), 0.54-0.43(\mathrm{~m}, 2 \mathrm{H}), 0.22-0.06(\mathrm{~m}, 2 \mathrm{H})$. 


\section{Synthesis of 2-(2,2-dichlorocyclopropyl)ethyl phenyl sulfone (2g)}

2-(2,2-Dichlorocyclopropyl)ethyl phenyl sulfone was prepared by dichlorocarbene addition to but-3enyl phenyl sulfone (2g'). ${ }^{[9]}$ But-3-enyl phenyl sulfone was prepared from sodium benzenesulfinate and 4-bromobut-1-ene in DMF at rt.

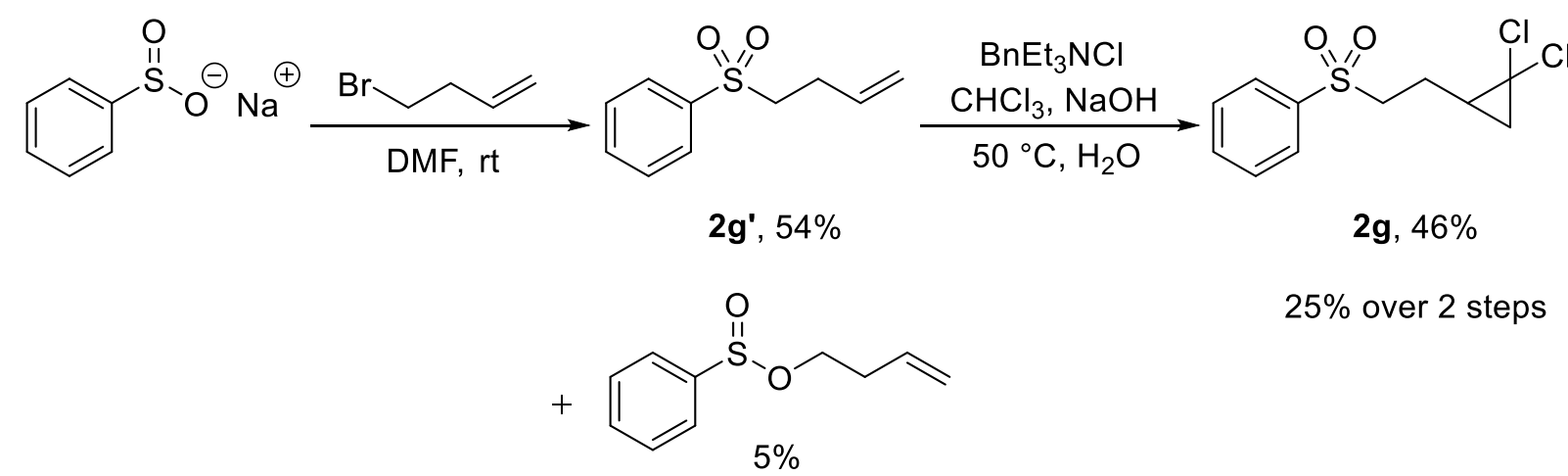

A $50 \mathrm{~mL}$ round-bottom flask was charged with sodium benzenesulfinate $(8.21 \mathrm{~g}, 50.0 \mathrm{mmol}, 1.00$ equiv.), DMF (20 mL), and immersed in water bath (rt). To the stirred reaction mixture 4-bromobut-1ene (8.11 g, $60.1 \mathrm{mmol}, 1.20$ equiv.) was added dropwise. After $23 \mathrm{~h} \mathrm{H}_{2} \mathrm{O}(100 \mathrm{~mL})$ was added, mixture was extracted with ethyl acetate $(3 \times 100 \mathrm{~mL})$, combined organic phases were washed with $\mathrm{LiCl}_{\mathrm{aq}}$ $(100 \mathrm{~mL}, 1.0 \mathrm{M}), \mathrm{Na}_{2} \mathrm{SO}_{3}$ aq $(100 \mathrm{~mL}, 10 \% \mathrm{w} / \mathrm{v})$, brine $(100 \mathrm{~mL})$, and dried over anhydrous $\mathrm{MgSO}_{4}$. The mixture was filtered, evaporated and separated with column chromatography (eluent: cyclohexane/ethyl acetate 6:1 to $2: 1)$ yielding $\mathbf{2 g}$ ' $(5.31 \mathrm{~g}, 27.1 \mathrm{mmol}, \mathbf{5 4 \%}$, more polar fraction) as a colorless oil, and but-1-en-4-yl benzenesulfinate $(511 \mathrm{mg}, 2.60 \mathrm{mmol}, \mathbf{5 \%}$, less polar fraction) as a pale yellow oil.

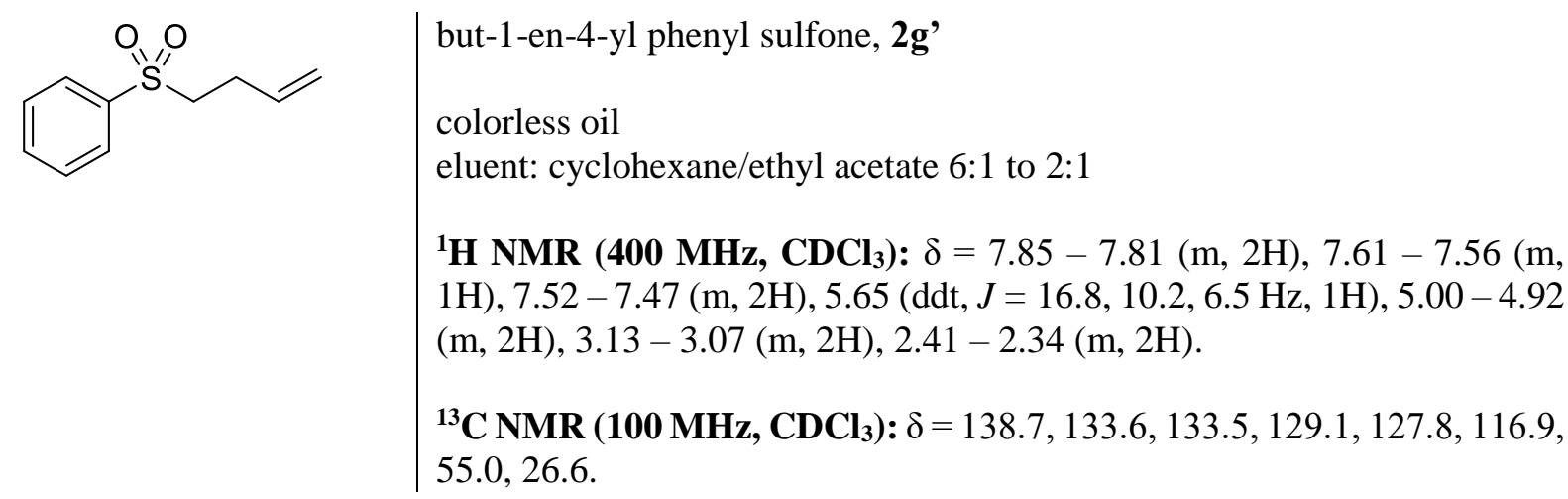

${ }^{1} \mathrm{H}$ NMR, ${ }^{13} \mathrm{C}$ NMR spectra were consistent with those reported in the literature. ${ }^{[10]}$ 
<smiles>C=CCCOS(=O)c1ccccc1</smiles>

but-1-en-4-yl benzenesulfinate

pale yellow oil

eluent: cyclohexane/ethyl acetate 6:1 to $2: 1$

${ }^{1} \mathbf{H}$ NMR (400 MHz, $\left.\mathbf{C D C l}_{3}\right): \delta=7.72-7.67(\mathrm{~m}, 2 \mathrm{H}), 7.55-7.48(\mathrm{~m}$, $3 \mathrm{H}), 5.71$ (ddt, $J=17.0,10.3,6.7 \mathrm{~Hz}, 1 \mathrm{H}), 5.10-5.02(\mathrm{~m}, 2 \mathrm{H}), 4.07(\mathrm{dt}, J$ $=9.9,6.8 \mathrm{~Hz}, 1 \mathrm{H}), 3.63(\mathrm{dt}, J=9.9,6.7 \mathrm{~Hz}, 1 \mathrm{H}), 2.37(\mathrm{qt}, J=6.7,1.4 \mathrm{~Hz}$, $2 \mathrm{H})$.

${ }^{13}$ C NMR (100 MHz, $\left.\mathbf{C D C l}_{3}\right): \delta=144.2,133.1,131.7,128.6,124.8,117.1$, 63.0, 33.6.

A $50 \mathrm{~mL}$ round-bottom flask was charged with $\mathbf{2 g}$ ' $(2.60 \mathrm{~g}, 13.3 \mathrm{mmol}, 1.00$ equiv.), benzyltriethylammonium chloride $(65.0 \mathrm{mg}, 0.285 \mathrm{mmol}, 2 \mathrm{~mol} \%)$, and $\mathrm{CHCl}_{3}(32 \mathrm{~mL})$. To vigorously stirred reaction mixture solution of $\mathrm{NaOH}\left(15.9 \mathrm{~g}, 397 \mathrm{mmol}, 29.8\right.$ equiv.) in $\mathrm{H}_{2} \mathrm{O}(16 \mathrm{~mL})$ was added, and mixture was heated to $50{ }^{\circ} \mathrm{C}$ (oil bath). After $60 \mathrm{~h}$, mixture was extracted with dichloromethane $(3 \times 50 \mathrm{~mL})$, combined organic phases were washed with $\mathrm{H}_{2} \mathrm{O}(2 \times 50 \mathrm{~mL})$, brine $(50 \mathrm{~mL})$, and dried over anhydrous $\mathrm{MgSO}_{4}$. The mixture was filtered, evaporated, and separated with column chromatography (eluent: cyclohexane/ethyl acetate $10: 1$ to $1: 3)$ yielding $\mathbf{2 g}(1.71 \mathrm{~g}, 6.13 \mathrm{mmol}, \mathbf{4 6 \%}$, $\mathbf{2 5 \%}$ over 2 steps) as a pale yellow solid.<smiles>O=S(=O)(CCC1CC1(Cl)Cl)c1ccccc1</smiles>$$
1
$$

NMR (400 MHz, $\left.\mathbf{C D C l}_{3}\right): \delta=7.93-7.88(\mathrm{~m}, 2 \mathrm{H}), 7.68-7.62(\mathrm{~m}$ $1 \mathrm{H}), 7.61-7.52(\mathrm{~m}, 2 \mathrm{H}), 3.35-3.17(\mathrm{~m}, 2 \mathrm{H}), 2.11-2.00(\mathrm{~m}, 1 \mathrm{H}), 1.90-$ $1.79(\mathrm{~m}, 1 \mathrm{H}), 1.69-1.55(\mathrm{~m}, 2 \mathrm{H}), 1.12-1.06(\mathrm{~m}, 1 \mathrm{H})$.

${ }^{13} \mathbf{C}$ NMR (100 MHz, $\left.\mathbf{C D C l}_{3}\right): \delta=138.8,133.9,129.4,128.0,60.2,54.7$, 28.6, 26.7, 24.0.

MS (EI): $\mathrm{m} / \mathrm{z}(\%)=283,281,279\left(1,1,2,[\mathrm{M}+\mathrm{H}]^{+}\right), 282,280,278(1,5$, 7, $\left.[\mathrm{M}]^{+}\right), 195\left(3,\left[\mathrm{M}-\mathrm{CHCl}_{2}\right]^{+}\right), 142,138,136\left(5,18,100,\left[\mathrm{C}_{5} \mathrm{H}_{6} \mathrm{Cl}_{2}\right]^{+}\right), 103$, $101\left(33,67,\left[\mathrm{C}_{5} \mathrm{H}_{6} \mathrm{Cl}\right]^{+}\right), 77\left(68,\left[\mathrm{C}_{6} \mathrm{H}_{5}\right]^{+}\right)$.

HRMS (EI): $\mathrm{m} / \mathrm{z}[\mathrm{M}]^{+}$calcd for $\mathrm{C}_{11} \mathrm{H}_{12} \mathrm{O}_{2} \mathrm{~S}^{35} \mathrm{Cl}_{2}$ : 277.9935; found: 277.9943. 


\section{Synthesis of (1-methyl-4-piperidinyl)methyl phenyl sulfone (2h)}

Preparation of (1-methyl-4-piperidinyl)methyl phenyl sulfide was performed according to Mitsunobu reaction protocol. ${ }^{[11]}$ Oxidation step was performed according to the reported procedure. ${ }^{[1]}$

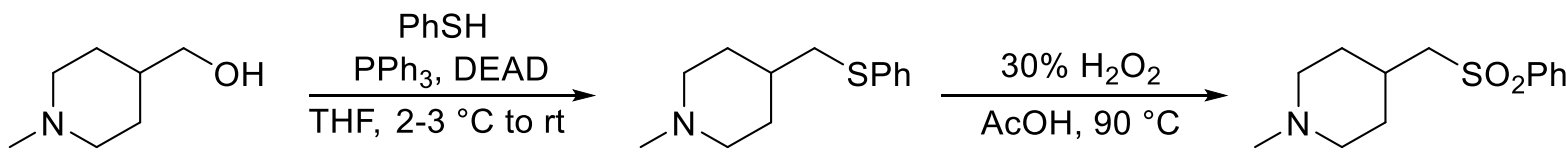

$2 \mathrm{~h}, 29 \%$

over 2 steps

A $100 \mathrm{~mL}$ Schlenk flask was charged with $\mathrm{PPh}_{3}(4.14 \mathrm{~g}, 15.8 \mathrm{mmol}, 1.05$ equiv.) and flushed with argon. Dry THF $(56 \mathrm{~mL})$ was added and mixture was immersed in ice/water bath $\left(2-3{ }^{\circ} \mathrm{C}\right)$. To the stirred reaction mixture DEAD (2.78 g, $15.9 \mathrm{mmol}, 1.06$ equiv.) was added dropwise and then mixture of 1-methyl-4-piperidinemethanol (1.94 g, $15.0 \mathrm{mmol}, 1.00$ equiv.) in dry THF (10 mL) was added. After $30 \mathrm{~min} \mathrm{PhSH}$ (1.98 g, $18.0 \mathrm{mmol}, 1.20$ equiv.) was added, and reaction mixture was stirred for $72 \mathrm{~h}$. Then mixture was evaporated and separated with column chromatography (eluent: ethyl acetate, then dichloromethane, then dichloromethane/MeOH 50:1 to 10:1) yielding crude (1-methyl-4piperidinyl)methyl phenyl sulfide. A $50 \mathrm{~mL}$ round-bottom flask was charged with crude (1-methyl-4piperidinyl)methyl phenyl sulfide, $\mathrm{AcOH}(10 \mathrm{~mL})$ was added, to the stirred reaction mixture $\mathrm{H}_{2} \mathrm{O}_{2}$ aq ( $3.07 \mathrm{~mL}, 30.1 \mathrm{mmol}, 30 \% \mathrm{v} / \mathrm{v}, 2.01$ equiv.) was added, and mixture was heated to $90{ }^{\circ} \mathrm{C}$ (oil bath). After $8 \mathrm{~h}$ mixture was evaporated with toluene $(3 \times 25 \mathrm{~mL}), \mathrm{K}_{2} \mathrm{CO}_{3}(2.07 \mathrm{~g}, 15.0 \mathrm{mmol}, 1.00$ equiv. $)$ and dichloromethane $(10 \mathrm{~mL})$ were added, and mixture was stirred overnight. Then, the mixture was passed through a pad of celite, evaporated, and separated with column chromatography (eluent: dichloromethane/MeOH 10:1 with 1\% v/v NEt 3 ) yielding $\mathbf{2 h}(1.11 \mathrm{~g}, 4.37 \mathrm{mmol}, \mathbf{2 9 \%}$ over 2 steps) as a yellow oil.

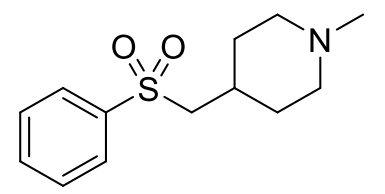

| (1-methyl-4-piperidinyl)methyl phenyl sulfone, $\mathbf{2 h}$

yellow oil

eluent: dichloromethane/MeOH 10:1 with $1 \% \mathrm{v} / \mathrm{v} \mathrm{NEt}_{3}$

${ }^{1} \mathbf{H}$ NMR (400 MHz, $\left.\mathbf{C D C l}_{3}\right): \delta=7.82-7.77(\mathrm{~m}, 2 \mathrm{H}), 7.57-7.52(\mathrm{~m}$, $1 \mathrm{H}), 7.50-7.41(\mathrm{~m}, 2 \mathrm{H}), 2.92(\mathrm{~d}, J=6.3 \mathrm{~Hz}, 2 \mathrm{H}), 2.75(\mathrm{~d}, J=12.1 \mathrm{~Hz}$, $2 \mathrm{H}), 2.18(\mathrm{~s}, 3 \mathrm{H}), 1.99-1.86(\mathrm{~m}, 3 \mathrm{H}), 1.82-1.74(\mathrm{~m}, 2 \mathrm{H}), 1.44-1.29(\mathrm{~m}$, $2 \mathrm{H})$.

${ }^{13} \mathrm{C}$ NMR (100 MHz, $\left.\mathbf{C D C l}_{3}\right): \delta=139.8,133.5,129.1,127.4,61.6,54.7$, $45.7,31.5,29.9$.

MS (EI): $\mathrm{m} / \mathrm{z}(\%)=254\left(28,[\mathrm{M}+\mathrm{H}]^{+}\right), 253\left(65,[\mathrm{M}]^{+}\right), 252\left(75,[\mathrm{M}-\mathrm{H}]^{+}\right)$, $238\left(18,\left[\mathrm{M}-\mathrm{CH}_{3}\right]^{+}\right), 112\left(100,\left[\mathrm{M}-\mathrm{PhSO}_{2}\right]^{+}\right)$.

HRMS (EI): $\mathrm{m} / \mathrm{z}$ [M] ${ }^{+}$calcd for $\mathrm{C}_{13} \mathrm{H}_{19} \mathrm{NO}_{2} \mathrm{~S}$ : 253.1137; found: 253.1127 . 


\section{Synthesis of isopropyl phenyl sulfone (2i)}

Isopropyl phenyl sulfone was prepared according to the reported procedure. ${ }^{[1]}$ Alkylation step was performed using $\mathrm{K}_{2} \mathrm{CO}_{3}$ in $\mathrm{MeCN}$.

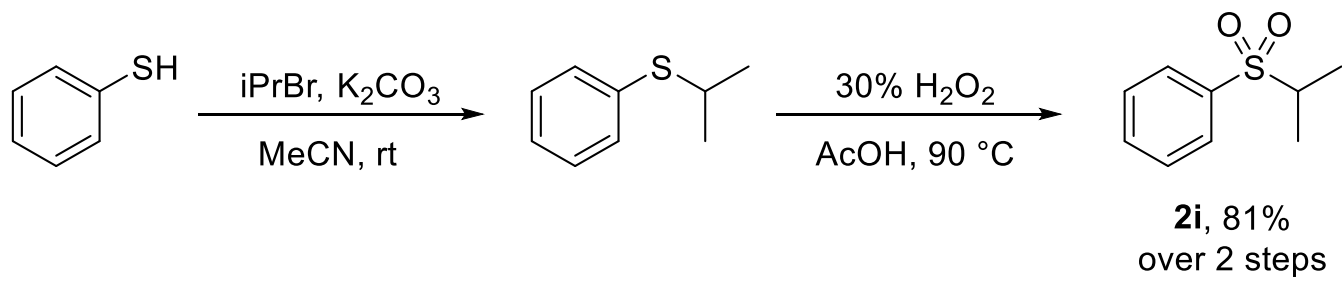

A $250 \mathrm{~mL}$ round-bottom flask was charged with $\mathrm{K}_{2} \mathrm{CO}_{3}$ (13.8 g, $100 \mathrm{mmol}, 1.96$ equiv.), MeCN (50 $\mathrm{mL}$ ) and immersed in water bath (rt). Thiophenol (5.63 g, $51.1 \mathrm{mmol}, 1.00$ equiv.) and isopropyl bromide ( $9.17 \mathrm{~g}, 75.0 \mathrm{mmol}, 1.47$ equiv.) were added, and mixture was vigorously stirred. After $26 \mathrm{~h}$ $\mathrm{H}_{2} \mathrm{O}(50 \mathrm{~mL})$ was added, $\mathrm{MeCN}$ was evaporated, and afterwards mixture was extracted with ethyl acetate $(3 \times 50 \mathrm{~mL})$. Combined organic phases were washed with $\mathrm{H}_{2} \mathrm{O}(50 \mathrm{~mL})$, brine $(50 \mathrm{~mL})$ and dried over anhydrous $\mathrm{MgSO}_{4}$. Mixture was filtered, evaporated, and placed in $100 \mathrm{~mL}$ round-bottom flask. Acetic acid $(20 \mathrm{~mL})$ was added, flask was heated to $50{ }^{\circ} \mathrm{C}$ (oil bath), to the stirred reaction mixture $\mathrm{H}_{2} \mathrm{O}_{2}$ aq $\left(15 \mathrm{~mL}, 147 \mathrm{mmol}, 30 \% \mathrm{v} / \mathrm{v}, 2.88\right.$ equiv.) was added, and the mixture was heated to $90{ }^{\circ} \mathrm{C}$ (oil bath). After $2 \mathrm{~h}$ mixture was poured onto water/ice $(200 \mathrm{~mL})$, solid $\mathrm{Na}_{2} \mathrm{CO}_{3}$ was added to $\mathrm{pH}=7$. Mixture was extracted with ethyl acetate $(3 \times 50 \mathrm{~mL})$, combined organic phases were washed with $\mathrm{Na}_{2} \mathrm{SO}_{3}$ aq $(50 \mathrm{~mL}, 10 \% \mathrm{w} / \mathrm{v})$, brine $(50 \mathrm{~mL})$ and dried over anhydrous $\mathrm{MgSO}_{4}$. Mixture was filtered, evaporated, and dried under vacuum yielding $2 \mathbf{i}(7.60 \mathrm{~g}, 41.2 \mathrm{mmol}, \mathbf{8 1 \%}$ over 2 steps) as a colorless oil.<smiles>CC(C)S(=O)(=O)c1ccccc1</smiles>
isopropyl phenyl sulfone, $\mathbf{2 i}$
colorless oil

${ }^{1}$ H NMR (400 MHz, $\left.\mathbf{C D C l}_{3}\right): \delta=7.84-7.78(\mathrm{~m}, 2 \mathrm{H}), 7.62-7.55(\mathrm{~m}$, $1 \mathrm{H}), 7.53-7.46(\mathrm{~m}, 2 \mathrm{H}), 3.13(\mathrm{hept}, J=6.9 \mathrm{~Hz}, 1 \mathrm{H}), 1.21(\mathrm{~d}, J=6.9 \mathrm{~Hz}$, $6 \mathrm{H})$.

${ }^{13} \mathrm{C}$ NMR (100 MHz, $\left.\mathbf{C D C l}_{3}\right): \delta=136.7,133.5,128.9,128.8,55.3,15.5$.

${ }^{1} \mathrm{H}$ NMR, ${ }^{13} \mathrm{C}$ NMR spectra were consistent with those reported in the literature. ${ }^{[12]}$ 


\subsection{Syntheses of neopentyl alkanesulfonates $(3 a-3 c, 3 e)$}

Neopentyl alkanesulfonates were prepared from corresponding sulfonyl chlorides and neopentanol, according to the reported procedure. ${ }^{[13]}$ Letter indexes of the compounds (3) refer to the same alkyl groups, as for alkyl phenyl sulfones (2). i.e.: $\mathbf{2} \mathbf{a}$ and $\mathbf{3 a}$ are methylating agents, $\mathbf{2} \mathbf{b}$ and $\mathbf{3 b}$ are ethylating agents, etc.

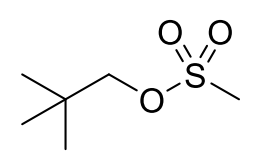

$3 a$

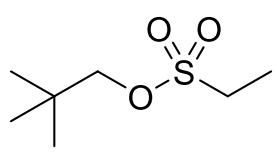

3b

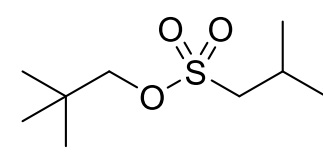

$3 c$<smiles>CCCCCCCS(=O)(=O)OCC(C)(C)C</smiles>

$3 e$

Synthesis of neopentyl methanesulfonate (3a)

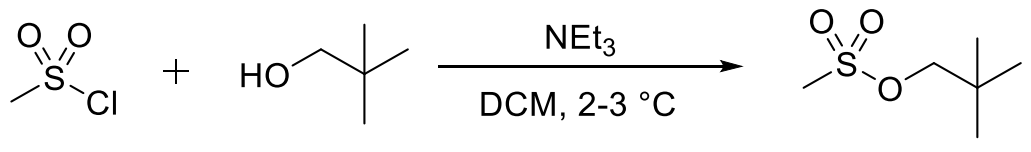

3a, $74 \%$

A $250 \mathrm{~mL}$ round-bottom flask was charged with neopentyl alcohol (4.43 g, $50.3 \mathrm{mmol}, 1.00$ equiv.), dichloromethane $(60 \mathrm{~mL})$, triethylamine $(5.80 \mathrm{~g}, 57.0 \mathrm{mmol}, 1.13$ equiv.), flushed with argon, and cooled in ice/water bath (ca. $\left.2-3{ }^{\circ} \mathrm{C}\right)$. To the stirred reaction mixture methanesulfonyl chloride $(6.49 \mathrm{~g}$, $56.7 \mathrm{mmol}, 1.13$ equiv.) was added dropwise. After $2.5 \mathrm{~h} \mathrm{H}_{2} \mathrm{O}(50 \mathrm{~mL})$ was added, phases were separated, and aqueous phase was extracted with ethyl acetate $(3 \times 50 \mathrm{~mL})$. Combined organic phases were washed with $\mathrm{H}_{2} \mathrm{O}(50 \mathrm{~mL})$, brine $(50 \mathrm{~mL})$, and dried over anhydrous $\mathrm{MgSO}_{4}$. Mixture was filtered, evaporated, and crude product was separated by distillation $\left(78^{\circ} \mathrm{C}, 4 \mathrm{mbar}\right)$ yielding $\mathbf{3 a}(6.17 \mathrm{~g}, 37.1$ mmol, $\mathbf{7 4 \%}$ ) as a colorless oil.<smiles>CC(C)(C)COS(C)(=O)=O</smiles>

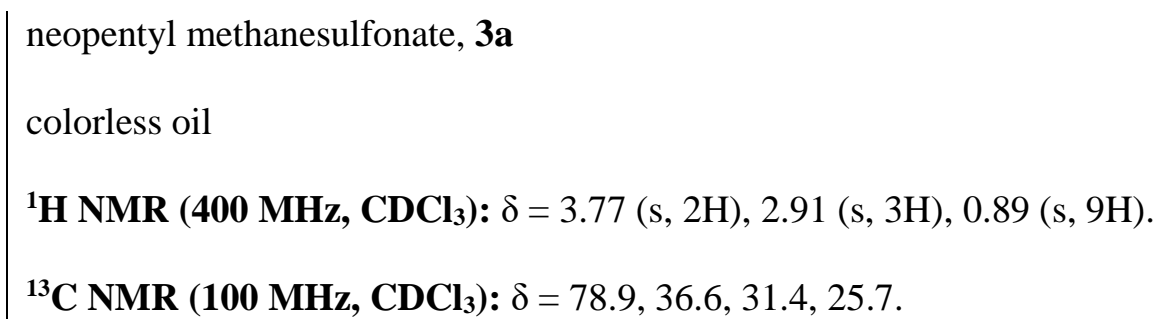

${ }^{1}$ H NMR (400 MHz, CDCl $): \delta=3.77$ (s, 2H), 2.91 (s, 3H), 0.89 (s, 9H).

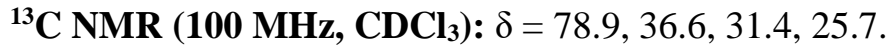

${ }^{1} \mathrm{H}$ NMR, ${ }^{13} \mathrm{C}$ NMR spectra were consistent with those reported in the literature. ${ }^{[1]}$ 


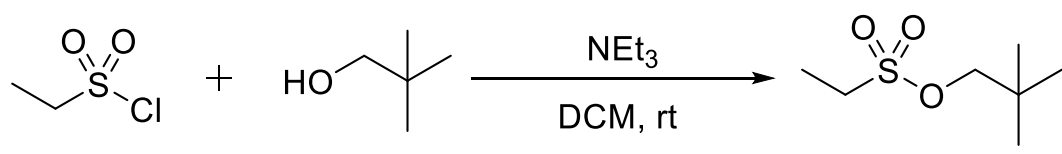

$3 b, 83 \%$

A $250 \mathrm{~mL}$ round-bottom flask was charged with neopentyl alcohol (3.56 g, $40.4 \mathrm{mmol}, 1.00$ equiv.), dichloromethane $(60 \mathrm{~mL})$, triethylamine $(5.81 \mathrm{~g}, 57.4 \mathrm{mmol}, 1.43$ equiv.), and immersed in water bath (rt). To the stirred reaction mixture ethanesulfonyl chloride $(5.23 \mathrm{~g}, 40.6 \mathrm{mmol}, 1.00$ equiv.) was added dropwise. After $2 \mathrm{~h} \mathrm{NH}_{4} \mathrm{Cl}_{\text {aq }}(50 \mathrm{~mL}, 10 \% \mathrm{w} / \mathrm{v})$ was added, phases were separated, and aqueous phase was extracted with ethyl acetate $(3 \times 50 \mathrm{~mL})$. Combined organic phases were washed with $\mathrm{H}_{2} \mathrm{O}(50$ $\mathrm{mL})$, brine $(50 \mathrm{~mL})$, and dried over anhydrous $\mathrm{MgSO}_{4}$. Mixture was filtered, evaporated, and product was distilled $\left(79^{\circ} \mathrm{C}, 3.3 \mathrm{mbar}\right)$ yielding $\mathbf{3 b}(6.03 \mathrm{~g}, 33.4 \mathrm{mmol}, \mathbf{8 3 \%})$ as a colorless oil.<smiles>CCS(=O)(=O)OCC(C)(C)C</smiles>

neopentyl ethanesulfonate, $\mathbf{3 b}$

colorless oil

${ }^{1} \mathbf{H}$ NMR (400 MHz, $\left.\mathbf{C D C l}_{3}\right): \delta=3.78(\mathrm{~s}, 2 \mathrm{H}), 3.06(\mathrm{q}, J=7.4 \mathrm{~Hz}, 2 \mathrm{H})$, $1.35(\mathrm{t}, J=7.4 \mathrm{~Hz}, 3 \mathrm{H}), 0.92(\mathrm{~s}, 9 \mathrm{H})$.

${ }^{13}$ C NMR (100 MHz, $\left.\mathbf{C D C l}_{3}\right): \delta=78.5,44.4,31.6,25.9,8.0$.

${ }^{1} \mathrm{H}$ NMR spectrum was consistent with that reported in the literature. ${ }^{[14]}$ 


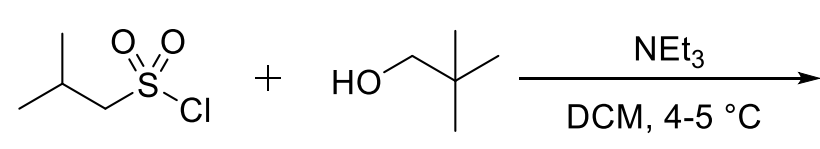<smiles>CC(C)CS(=O)(=O)OCC(C)(C)C</smiles>

3c, $77 \%$

A $250 \mathrm{~mL}$ round-bottom flask was charged with neopentyl alcohol (2.82 g, $32.0 \mathrm{mmol}, 1.02$ equiv.), dichloromethane $(48 \mathrm{~mL})$, isobutanesulfonyl chloride $(5.01 \mathrm{~g}, 31.3 \mathrm{mmol}, 1.00$ equiv.), and immersed in water/ice bath $\left(4-5^{\circ} \mathrm{C}\right)$. To the stirred reaction mixture triethylamine ( $4.50 \mathrm{~g}, 44.5 \mathrm{mmol}, 1.42$ equiv.) was added dropwise. After $2 \mathrm{~h} \mathrm{H}_{2} \mathrm{O}(50 \mathrm{~mL})$ was added, phases were separated, and aqueous phase was extracted with ethyl acetate $(3 \times 50 \mathrm{~mL})$. Combined organic phases were washed with $\mathrm{H}_{2} \mathrm{O}(50 \mathrm{~mL})$, brine $(50 \mathrm{~mL})$, and dried over anhydrous $\mathrm{MgSO}_{4}$. Mixture was filtered, evaporated, and product was distilled $\left(93-95{ }^{\circ} \mathrm{C}, 4.0 \mathrm{mbar}\right)$ yielding $\mathbf{3 c}(5.05 \mathrm{~g}, 24.2 \mathrm{mmol}, \mathbf{7 7 \%})$ as a colorless oil.<smiles>CC(C)CS(=O)(=O)OCC(C)(C)C</smiles>

neopentyl isobutanesulfonate, $\mathbf{3 c}$

colorless oil

${ }^{1} \mathbf{H}$ NMR (400 MHz, CDCl $): \delta=3.81(\mathrm{~s}, 2 \mathrm{H}), 2.95(\mathrm{~d}, J=6.6 \mathrm{~Hz}, 2 \mathrm{H})$, $2.34-2.19(\mathrm{~m}, 1 \mathrm{H}), 1.08(\mathrm{~d}, J=6.8 \mathrm{~Hz}, 6 \mathrm{H}), 0.94(\mathrm{~s}, 9 \mathrm{H})$.

${ }^{13}$ C NMR (100 MHz, $\left.\mathbf{C D C l}_{3}\right): \delta=78.2,57.6,31.6,26.0,24.8,22.3$.

HRMS (ESI): $\mathrm{m} / \mathrm{z}$ [M+Na] ${ }^{+}$calcd for $\mathrm{C}_{9} \mathrm{H}_{20} \mathrm{O}_{3} \mathrm{SNa}$ : 231.1031; found: 231.1032 . 


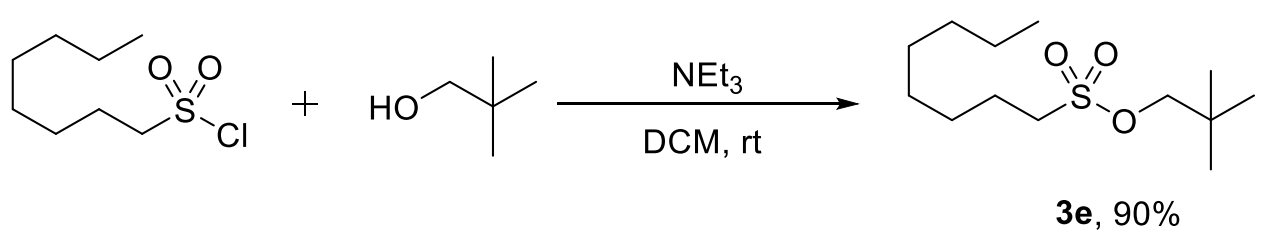

A $250 \mathrm{~mL}$ round-bottom flask was charged with neopentyl alcohol (4.41 g, $50.0 \mathrm{mmol}, 1.00$ equiv.), dichloromethane $(60 \mathrm{~mL})$, triethylamine $(7.11 \mathrm{~g}, 70.3 \mathrm{mmol}, 1.41$ equiv.), flushed with argon, and immersed in water bath (rt). To the stirred reaction mixture octanesulfonyl chloride (10.6 g, $50.0 \mathrm{mmol}$, 1.00 equiv.) was added dropwise. After $2 \mathrm{~h} \mathrm{H}_{2} \mathrm{O}(50 \mathrm{~mL})$ was added, phases were separated, and aqueous phase was extracted with ethyl acetate $(3 \times 50 \mathrm{~mL})$. Combined organic phases were washed with $\mathrm{H}_{2} \mathrm{O}$ $(50 \mathrm{~mL})$, brine $(50 \mathrm{~mL})$, and dried over anhydrous $\mathrm{MgSO}_{4}$. Mixture was filtered, evaporated, and product was separated with column chromatography (eluent: cyclohexane, then cyclohexane/ethyl acetate 20:1 to $10: 1)$ yielding $3 \mathbf{e}(11.9 \mathrm{~g}, 45.2 \mathrm{mmol}, \mathbf{9 0 \%})$ as a pale yellow oil.<smiles>CCCCCCCCS(=O)(=O)OCC(C)(C)C</smiles>

llow oi eluent: cyclohexane, then cyclohexane/ethyl acetate $20: 1$ to $10: 1$

${ }^{1}$ H NMR (400 MHz, CDCl $\left.\mathbf{~}_{3}\right): \delta=3.79$ (s, 2H), $3.05-3.00$ (m, 2H), 1.84 $-1.72(\mathrm{~m}, 2 \mathrm{H}), 1.42-1.31(\mathrm{~m}, 2 \mathrm{H}), 1.31-1.16(\mathrm{~m}, 8 \mathrm{H}), 0.92(\mathrm{~s}, 9 \mathrm{H}), 0.81$ $(\mathrm{t}, J=6.9 \mathrm{~Hz}, 3 \mathrm{H})$.

${ }^{13} \mathbf{C}$ NMR (100 MHz, $\left.\mathbf{C D C l}_{3}\right): \delta=78.3,49.9,31.6,31.5,28.8,28.7,28.0$, 25.9, 23.3, 22.4, 13.9.

${ }^{1} \mathrm{H}$ NMR, ${ }^{13} \mathrm{C}$ NMR spectra were consistent with those reported in the literature. ${ }^{[13]}$ 


\subsection{Syntheses of nitroarenes (1a-1i)}

Syntheses of nitroarenes $\mathbf{1 d}, \mathbf{1 f}$, and $\mathbf{1 h}$ were described below. Other nitroarenes were purchased from commercial suppliers (see the General Information).<smiles>O=[N+]([O-])c1cccnc1</smiles>

$1 \mathrm{a}$<smiles>COc1ccc([N+](=O)[O-])cn1</smiles><smiles>O=[N+]([O-])c1cccs1</smiles>

$1 \mathrm{~g}$<smiles>COc1ccncc1[N+](=O)[O-]</smiles><smiles>O=[N+]([O-])c1ccc(Sc2ccccc2)nc1</smiles><smiles>O=[N+]([O-])c1ccc(Cl)nc1</smiles>

$1 f$<smiles>O=[N+]([O-])c1ccc(Oc2c(Cl)cc(Cl)cc2Cl)nc1</smiles><smiles>O=[N+]([O-])c1cnc2ccccc2c1</smiles>

$1 \mathrm{~h}$<smiles>O=[N+]([O-])c1ccc(Cl)cc1</smiles>

$1 i$ 


\section{Synthesis of 5-nitro-2-(phenylthio)pyridine (1d)}

5-Nitro-2-(phenylthio)pyridine (1d) was prepared from 2-bromo-5-nitropyridine and thiophenol using $\mathrm{NEt}_{3}$ in DMF at rt.

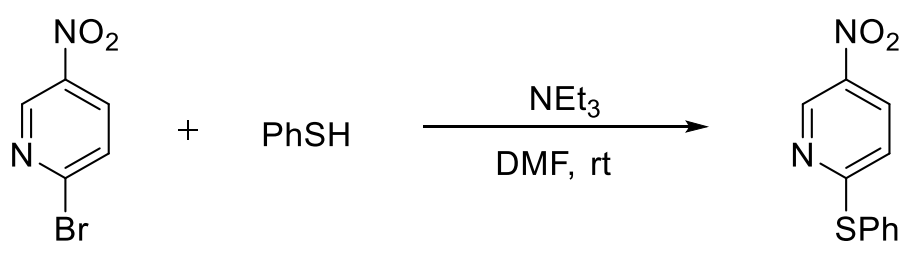

$1 d, 86 \%$

A $30 \mathrm{~mL}$ Schlenk flask was charged with 2-bromo-5-nitropyridine (2.03 g, $10.0 \mathrm{mmol}, 1.00$ equiv.), thiophenol (1.16 g, $10.5 \mathrm{mmol}, 1.05$ equiv.), and flushed with argon. Dry DMF (12 mL) was added, and Schlenk flask was immersed in water bath (rt). To the stirred reaction mixture $\mathrm{NEt}_{3}(1.52 \mathrm{~g}, 15.1 \mathrm{mmol}$, 1.51 equiv.) was added. After $30 \mathrm{~min}$ the mixture was transferred to separatory funnel, $\mathrm{NH}_{4} \mathrm{Cl}_{\mathrm{aq}}(50 \mathrm{~mL}$, $10 \% \mathrm{w} / \mathrm{v})$ was added, mixture was extracted with ethyl acetate $(3 \times 50 \mathrm{~mL})$, combined organic phases were washed with $\mathrm{LiCl}_{\mathrm{aq}}(50 \mathrm{~mL}, 1.0 \mathrm{M}), \mathrm{H}_{2} \mathrm{O}(50 \mathrm{~mL})$, brine $(50 \mathrm{~mL})$, and dried over anhydrous $\mathrm{MgSO}_{4}$. Mixture was filtered, evaporated, and recrystallized from dichloromethane/heptane mixture yielding $1 \mathrm{~d}(2.00 \mathrm{~g}, 8.63 \mathrm{mmol}, \mathbf{8 6 \%})$ as off-white crystals.<smiles>O=[N+]([O-])c1ccc(Sc2ccccc2)nc1</smiles>

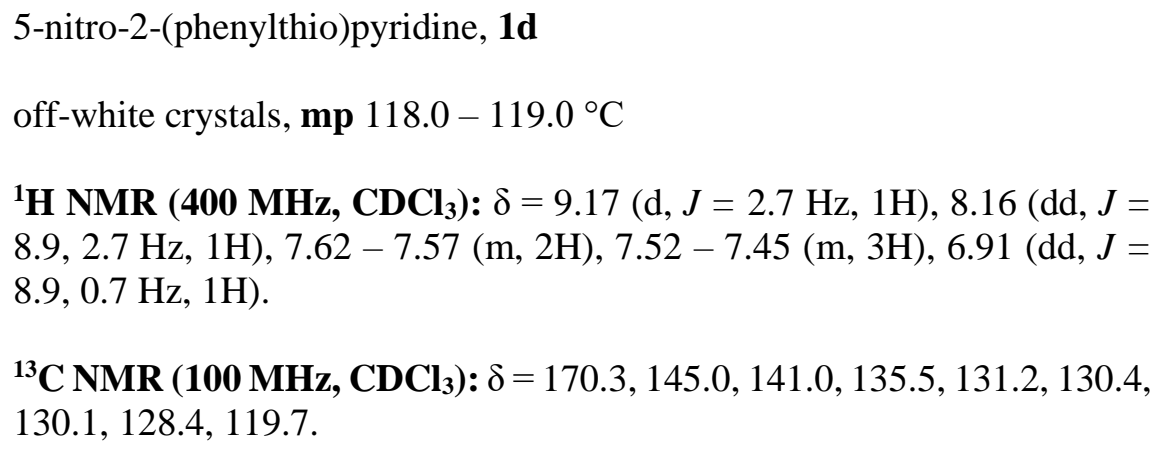

${ }^{1} \mathbf{H}$ NMR (400 MHz, $\left.\mathbf{C D C l}_{3}\right): \delta=9.17(\mathrm{~d}, J=2.7 \mathrm{~Hz}, 1 \mathrm{H}), 8.16(\mathrm{dd}, J=$ $8.9,2.7 \mathrm{~Hz}, 1 \mathrm{H}), 7.62-7.57(\mathrm{~m}, 2 \mathrm{H}), 7.52-7.45(\mathrm{~m}, 3 \mathrm{H}), 6.91$ (dd, $J=$ $8.9,0.7 \mathrm{~Hz}, 1 \mathrm{H})$

${ }^{13}$ C NMR (100 MHz, CDCl $): ~ \delta=170.3,145.0,141.0,135.5,131.2,130.4$, $130.1,128.4,119.7$.

${ }^{1} \mathrm{H}$ NMR, ${ }^{13} \mathrm{C}$ NMR spectra ${ }^{[15]}$ and $\mathrm{mp}^{[16]}$ were consistent with those reported in the literature. 


\section{Synthesis of 2-(2,4,6-trichlorophenoxy)-5-nitropyridine (1f)}

2-(2,4,6-Trichlorophenoxy)-5-nitropyridine (1f) was prepared according to modified procedure reported in the literature. ${ }^{[17]}$

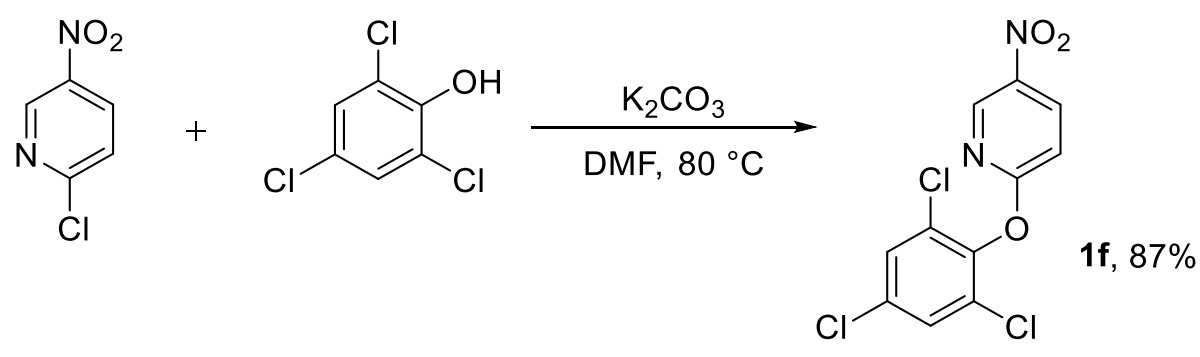

A $100 \mathrm{~mL}$ round-bottom flask was charged with $\mathrm{K}_{2} \mathrm{CO}_{3}(8.31 \mathrm{~g}, 60.1 \mathrm{mmol}, 3.00$ equiv.), dried under vacuum, and flushed with argon. Solution of 2-chloro-5-nitropyridine ( $3.17 \mathrm{~g}, 20.0 \mathrm{mmol}, 1.00$ equiv.) and 2,4,6-trichlorophenol ( $4.15 \mathrm{~g}, 21.0 \mathrm{mmol}, 1.05$ equiv.) in dry DMF $(20 \mathrm{~mL})$ was added, and mixture was stirred at $80{ }^{\circ} \mathrm{C}$ (oil bath). After $20 \mathrm{~h}$ mixture was transferred to separatory funnel, $\mathrm{H}_{2} \mathrm{O}(50 \mathrm{~mL})$ was added, mixture was extracted with ethyl acetate $(3 \times 50 \mathrm{~mL})$, combined organic phases were washed with $\mathrm{LiCl}_{\mathrm{aq}}(50 \mathrm{~mL}, 1.0 \mathrm{M}), \mathrm{H}_{2} \mathrm{O}(50 \mathrm{~mL})$, brine $(50 \mathrm{~mL})$, and dried over anhydrous $\mathrm{MgSO}_{4}$. Mixture was filtered, evaporated, and recrystallized from dichloromethane/heptane mixture yielding in three crops $\mathbf{1 f}(5.59 \mathrm{~g}, 17.5 \mathrm{mmol}, \mathbf{8 7 \%})$ as light brown crystals.<smiles>O=[N+]([O-])c1ccc(Oc2c(Cl)cc(Cl)cc2Cl)nc1</smiles>

2-(2,4,6-trichlorophenoxy)-5-nitropyridine, $\mathbf{1 f}$

light brown crystals, $\mathbf{m p} 104.0-106.0^{\circ} \mathrm{C}$

${ }^{1}$ H NMR (400 MHz, $\left.\mathbf{C D C l}_{3}\right): \delta=8.94(\mathrm{dd}, J=2.8,0.6 \mathrm{~Hz}, 1 \mathrm{H}), 8.54(\mathrm{dd}$, $J=9.0,2.8 \mathrm{~Hz}, 1 \mathrm{H}), 7.41(\mathrm{~s}, 2 \mathrm{H}), 7.22(\mathrm{dd}, J=9.0,0.6 \mathrm{~Hz}, 1 \mathrm{H})$.

${ }^{13}$ C NMR (100 MHz, $\left.\mathbf{C D C l}_{3}\right): \delta=164.4,144.6,144.5,141.1,135.4,132.0$, $129.9,128.8,111.0$.

MS (EI): $\mathrm{m} / \mathrm{z}(\%)=287,285,283\left(14,77,100,[\mathrm{M}-\mathrm{Cl}]^{+}\right), 241,239,237$ $\left(6,39,59,\left[\mathrm{M}-\mathrm{Cl}-\mathrm{NO}_{2}\right]^{+}\right)$.

HRMS (EI): $\mathrm{m} / \mathrm{z}[\mathrm{M}]^{+}$calcd for $\mathrm{C}_{11} \mathrm{H}_{5} \mathrm{~N}_{2} \mathrm{O}_{3}{ }^{35} \mathrm{Cl}_{3}$ : 317.9366; found: 319.9360 . 


\title{
Synthesis of 3-nitroquinoline (1h)
}

3-Nitroquinoline (1h) was prepared according to procedure reported in the literature. ${ }^{[18]}$

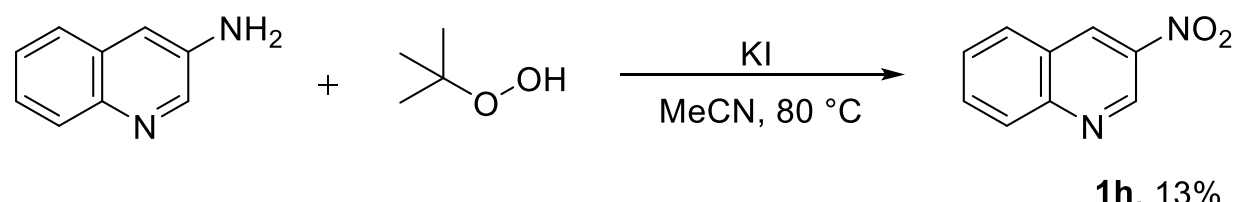

A $100 \mathrm{~mL}$ round-bottom flask was charged with 3 -aminoquinoline $(2.31 \mathrm{~g}, 16.0 \mathrm{mmol}, 1.00$ equiv.), KI (133 $\mathrm{mg}, 0.80 \mathrm{mmol}, 5 \mathrm{~mol} \%)$ and $\mathrm{MeCN}(40 \mathrm{~mL})$. To the stirred reaction mixture solution of tert-butyl hydroperoxide (7.85 g, $61.0 \mathrm{mmol}, 3.80$ equiv., $70 \%$ w/w in $\left.\mathrm{H}_{2} \mathrm{O}\right)$ in $\mathrm{MeCN}(8 \mathrm{~mL})$ was added dropwise over $25 \mathrm{~min}$, and then mixture was heated to $80{ }^{\circ} \mathrm{C}$ (oil bath). After $24 \mathrm{~h} \mathrm{Na}_{2} \mathrm{~S}_{2} \mathrm{O}_{3}$ aq $(100 \mathrm{~mL}$, sat.) was added, mixture was extracted with ethyl acetate $(3 \times 100 \mathrm{~mL})$, combined organic phases were washed with brine $(50 \mathrm{~mL})$, and dried over anhydrous $\mathrm{MgSO}_{4}$. Mixture was filtered, evaporated, and product was separated with column chromatography (eluent: cyclohexane/ethyl acetate 20:1 to 1:1) yielding $\mathbf{1 h}$ (357 $\mathrm{mg}, 2.05 \mathrm{mmol}, \mathbf{1 3 \%}$ ) as brown crystals.<smiles>O=[N+]([O-])c1cnc2ccccc2c1</smiles>

\author{
3-nitroquinoline, $\mathbf{1 h}$ \\ brown crystals, $\mathbf{m p} 126.5-127.5^{\circ} \mathrm{C}$ \\ eluent: cyclohexane/ethyl acetate $20: 1$ to $1: 1$
}

${ }^{1} \mathbf{H}$ NMR $\left(400 \mathrm{MHz}, \mathbf{C D C l}_{3}\right): \delta=9.60(\mathrm{~d}, J=2.5 \mathrm{~Hz}, 1 \mathrm{H}), 9.00(\mathrm{~d}, J=$ $2.5 \mathrm{~Hz}, 1 \mathrm{H}), 8.20(\mathrm{~d}, J=8.5 \mathrm{~Hz}, 1 \mathrm{H}), 8.01(\mathrm{~d}, J=8.4 \mathrm{~Hz}, 1 \mathrm{H}), 7.96-7.87$ $(\mathrm{m}, 1 \mathrm{H}), 7.75-7.66(\mathrm{~m}, 1 \mathrm{H})$.

${ }^{13} \mathbf{C}$ NMR (100 MHz, $\left.\mathbf{C D C l}_{3}\right): \delta=150.0,144.0,140.9,133.4,132.2,129.8$, $129.7,128.8,125.9$.

${ }^{1} \mathrm{H}$ NMR, ${ }^{13} \mathrm{C}$ NMR spectra ${ }^{[19]}$ and $\mathrm{mp}^{[20]}$ were consistent with those reported in the literature. 


\section{Alkylation of nitroarenes}

\subsection{General Procedure for alkylation of nitroarenes}<smiles>O=[N+]([O-])C1=C[C+]=CN=C1</smiles><smiles></smiles>

alkyl sulfone or

nitroarene alkanesulfonate

1

2 or 3<smiles>[R]Cc1ccncc1[N+](=O)[O-]</smiles>

alkylated

nitroarenes

4

A $30 \mathrm{~mL}$ Schlenk flask was charged with nitroarene (1) (2.00 mmol, 1.00 equiv.), carbanion precursor (2 or 3) (2.40 mmol, 1.20 equiv.) and flushed with argon. Dry DMF (6 mL) was added, and after dissolution of substrates mixture was cooled to $-40^{\circ} \mathrm{C}$.* To the stirred reaction mixture KHMDS (5.00 mmol, 2.50 equiv., solution in $\mathrm{THF}^{* *}$ ) was added rapidly in one portion. After $30 \mathrm{~min}$ reaction mixture was quenched with $\mathrm{NH}_{4} \mathrm{Cl}_{\mathrm{aq}}(10 \mathrm{~mL}, 10 \% \mathrm{w} / \mathrm{v})$. Mixture was transferred to separatory funnel, $\mathrm{NH}_{4} \mathrm{Cl}_{\mathrm{aq}}$ $(50 \mathrm{~mL}, 10 \% \mathrm{w} / \mathrm{v})$ was added, mixture was extracted with ethyl acetate $(3 \times 50 \mathrm{~mL})$, combined organic phases were washed with $\mathrm{LiCl}_{\mathrm{aq}}(50 \mathrm{~mL}, 1.0 \mathrm{M}), \mathrm{H}_{2} \mathrm{O}(50 \mathrm{~mL})$, brine $(50 \mathrm{~mL})$, and dried over anhydrous $\mathrm{MgSO}_{4}$. Mixture was filtered, evaporated and separated with column chromatography (length $=\mathrm{ca}$. 40 $50 \mathrm{~cm}, \varnothing=3 \mathrm{~cm}, 250-350 \mathrm{~mL}$ of silica gel), and eluted with eluents indicated hereinafter.

* - methylation and ethylation of 3-nitropyridine (1a) were performed under slightly different conditions (see Table on next page)

** - for concentrations of commercially-available solutions of KHMDS in THF used in this study see the General Information 


\subsection{Preparative alkylation of nitroarenes}

\begin{tabular}{|c|c|c|c|c|c|c|c|}
\hline \multirow[b]{2}{*}{ entry } & \multicolumn{3}{|c|}{ conditions } & \multicolumn{4}{|c|}{ yields [\%] } \\
\hline & nitroarene & $\begin{array}{l}\text { carbanion } \\
\text { precursor }\end{array}$ & $\begin{array}{c}\text { variation } \\
\text { from General } \\
\text { Procedure }\end{array}$ & alkylated products & $\sigma^{\mathrm{H}}$-adduct & $\begin{array}{l}\text { nitroarene } \\
\text { recovered }\end{array}$ & side products \\
\hline 1 & 1a & $2 a$ & - & $\mathbf{4 a}, 12 \%$ & - & $17 \%$ & $13 \%{ }^{\mathrm{a}} ; \mathbf{6 c}^{\mathrm{b}}$ \\
\hline 2 & $1 \mathrm{a}$ & $3 a$ & - & $4 \mathbf{a}, 20 \%$ & - & $10 \%$ & $5 \%$; $6 \mathbf{6 c}, 24 \%$ \\
\hline 3 & $1 \mathrm{a}$ & 3a & $-60{ }^{\circ} \mathrm{C}, 3 \mathrm{~min}$ & $\mathbf{4 a}, 38 \%$ & - & $5 \%$ & $7 \% \%^{\mathrm{a}} ; \mathbf{6}^{\mathrm{b}}$ \\
\hline 4 & $1 \mathbf{a}$ & $2 \mathbf{b}$ & - & $4 b, 28 \%$ & - & $4 \%$ & - \\
\hline 5 & $1 \mathrm{a}$ & $2 \mathbf{b}$ & $3 \mathrm{~min}$ & $4 b, 35 \%$ & $15 \%^{\mathrm{c}}$ & $5 \%$ & $-d$ \\
\hline 6 & 1a & $2 c$ & - & $4 \mathbf{c}, 71 \% ; 4 \mathbf{c}, 10 \%$ & $2 \%^{c}$ & $1 \%$ & $-d$ \\
\hline 7 & $1 \mathbf{a}$ & $3 c$ & - & $\mathbf{4 c}, 73 \% ; \mathbf{4 c}, 9 \%$ & - & $1 \%$ & $-\mathrm{d}$ \\
\hline 8 & 1a & $2 d$ & - & $\mathbf{4 d}, 70 \%$ & $6 \% c$ & - & $-d$ \\
\hline 9 & 1a & $3 e$ & - & $4 e, 71 \% ; 4 e^{\prime}, 9 \%$ & - & - & 6d, $4 \% ;-{ }^{d}$ \\
\hline 10 & $1 \mathbf{a}$ & $2 \mathrm{e}$ & - & $4 \mathbf{e}, 53 \% ; 4 e^{\prime}, 10 \%$ & - & $-\mathrm{b}$ & 6d, $2 \% ;-^{d}$ \\
\hline 11 & $1 \mathbf{a}$ & $2 f$ & - & $\mathbf{4 f}, 72 \% ; \mathbf{4 f}, 9 \%$ & - & - & $-\mathrm{d}$ \\
\hline 12 & $1 \mathrm{a}$ & $2 \mathrm{~g}$ & - & $4 \mathrm{~g}, 64 \%$ & - & - & $-d$ \\
\hline 13 & $1 \mathrm{a}$ & $2 \mathrm{~h}$ & - & 4h, $61 \%$ & - & $4 \%$ & $-\mathrm{e}$ \\
\hline 14 & $1 \mathrm{~b}$ & $3 a$ & - & $4 \mathbf{i}, 54 \%$ & - & - & - \\
\hline 15 & $1 b$ & $3 e$ & - & $4 \mathbf{j}, 55 \%$ & 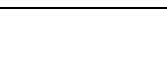 & 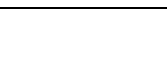 & $-d$ \\
\hline 16 & $1 \mathrm{c}$ & $3 e$ & - & $\mathbf{4 k}, 40 \% ; \mathbf{4 k}, 14 \%$ & - & - & $-{ }^{f}$ \\
\hline 17 & 1d & $2 c$ & - & $41,69 \%$ & $4 \%^{c}$ & $4 \%$ & $-\mathrm{d}$ \\
\hline 18 & 1d & $2 d$ & - & $4 \mathbf{m}, 86 \% ; 4 \mathbf{m}, 5 \%$ & - & $3 \%$ & - \\
\hline 19 & 1d & $3 e$ & - & $4 n, 64 \% ; 4 n ', 6 \%$ & - & - & - \\
\hline 20 & 1d & $2 f$ & - & 4o, 73\%; 4o', 8\% & $3 \%^{\mathrm{c}}$ & - & - \\
\hline 21 & 1d & $2 \mathrm{~g}$ & - & $\mathbf{4 p}, 53 \% ; \mathbf{4 p}, 9 \%$ & - & $3 \%$ & - \\
\hline 22 & $1 \mathrm{e}$ & $3 e$ & - & $\mathbf{4 q}, 36 \%$ & - & $3 \%$ & $3 \%^{\mathrm{d}}$ \\
\hline 23 & 1f & $3 e$ & - & $4 r, 50 \% ; 4 r^{\prime}, 26 \%$ & - & - & - \\
\hline 24 & $1 \mathrm{~g}$ & $2 c$ & - & $4 \mathrm{~s}, 55 \%$ & - & - & - \\
\hline 25 & $1 \mathrm{~g}$ & $3 e$ & - & $4 \mathbf{t}, 58 \%$ & - & - & - \\
\hline 26 & $1 \mathrm{a}$ & $2 \mathrm{i}$ & - & - & 5a, $43 \%^{\mathrm{g}}$ & - & - \\
\hline 27 & 1h & $2 \mathbf{b}$ & - & $5 \%^{\mathrm{h}}$ & $\mathbf{5 b}, 68 \%$ & $18 \%$ & - \\
\hline 28 & $1 \mathrm{~h}$ & $2 a$ & - & $\mathbf{4 u}, 79 \%$ & - & $4 \%$ & - \\
\hline 29 & 1h & $2 \mathbf{i}$ & - & - & $5 \mathbf{c}, 11 \%$ & $85 \%$ & - \\
\hline 30 & $\mathbf{1 i}$ & $3 e$ & - & $4 \mathbf{v}, 15 \%$ & - & $8 \%$ & $\mathbf{6 a}, 39 \% ; \mathbf{1 0}, 10 \%$ \\
\hline 31 & $\mathbf{1 i}$ & $\mathbf{3 b}$ & - & - & - & $28 \%$ & $\mathbf{6 b}, 23 \%^{\mathrm{i}}$ \\
\hline
\end{tabular}

a - ONSH (Oxidative Nucleophilic Substitution of Hydrogen) type product was formed, pure analytical sample was not available

$\mathrm{b}$ - due to sample contamination yield was not established

$\mathrm{c}-$ pure analytical sample was not available

$\mathrm{d}-\leq 5 \%$ of isomeric products, pure analytical samples were not available

e $-9 \%$ of 2-((1-methyl-4-piperidinyl)methyl)-5-nitropyridine was formed, pure analytical sample was not available

$\mathrm{f}-\mathrm{ca} .17 \%$ of $(E)$-oct-1-enylated products were formed, pure analytical samples were not available

$\mathrm{g}-\leq 5 \%$ of isomeric $\sigma$-adducts were formed, pure analytical samples were not available

$\mathrm{h}$ - 4-ethyl-3-nitroquinoline was formed, pure analytical sample was not available

$\mathrm{i}$ - ca. $18 \%$ of 2-vinyl-4-chloronitrobenzene was formed, pure analytical sample was not available 


\subsection{Informations concerning isolation of nitroarenes}

- Reactions were carried out in a Schlenk flask $(\varnothing 30 \times 92 \mathrm{~mm})$ and stirred with magnetic bar (fish, $\varnothing 10 \times 20 \mathrm{~mm}$ ) at $300 \mathrm{rpm}$,

- Solution of KHMDS in THF was added rapidly (during ca. 3-5 s) into vortex of a stirred reaction mixture. For concentrations of commercially-available solutions of KHMDS in THF used in this study see the General Information,

- Nitroarenes $\mathbf{1 b}, \mathbf{1 d}$, and $\mathbf{1 h}$ precipitate from DMF while cooling to $-40{ }^{\circ} \mathrm{C}$, however addition of KHMDS in THF makes the reaction mixture homogenous,

- In most cases it is crucial to use cyclohexane/toluene (for isolation of less polar products) or toluene/ethyl acetate (for isolation of more polar products) mixtures as eluent in column chromatography, while other popular eluents like cyclohexane, cyclohexane/ethyl acetate, cyclohexane/diethyl ether are less efficient (even if they might provide better separation at TLC),

- In general, products alkylated para to the $\mathrm{NO}_{2}$ group are eluted faster (are less polar) than orto isomers (eluted in cyclohexane/toluene and toluene/ethyl acetate mixtures). 


\subsection{Characterization of the alkylated products}<smiles>Cc1ccncc1[N+](=O)[O-]</smiles>

4-methyl-3-nitropyridine, $4 \mathbf{a}$

Yield:

$56 \mathrm{mg}(0.41 \mathrm{mmol}, \mathbf{2 0 \%})$ from $\mathbf{1 a}(251 \mathrm{mg}, 2.02 \mathrm{mmol})$ and 3a $35 \mathrm{mg}(0.25 \mathrm{mmol}, \mathbf{1 2 \%})$ from $\mathbf{1 a}(252 \mathrm{mg}, 2.03 \mathrm{mmol})$ and $\mathbf{2 a}$ $104 \mathrm{mg}(0.76 \mathrm{mmol}, \mathbf{3 8 \%})$ from $\mathbf{1 a}(248 \mathrm{mg}, 2.00 \mathrm{mmol})$ and $\mathbf{3 a}$ at $\mathbf{- 6 0}{ }^{\circ} \mathbf{C}$, 3 min

yellow oil

eluent: toluene/ethyl acetate 50:1 to 2:1

${ }^{1}$ H NMR (400 MHz, CDCl $): \delta=9.13(\mathrm{~s}, 1 \mathrm{H}), 8.62(\mathrm{~d}, J=5.0 \mathrm{~Hz}, 1 \mathrm{H})$, $7.29(\mathrm{~d}, J=5.0 \mathrm{~Hz}, 1 \mathrm{H}), 2.62(\mathrm{~s}, 3 \mathrm{H})$.

${ }^{13}$ C NMR (100 MHz, $\mathbf{C D C l}_{3}$ ): $\delta=152.9,145.8$ (ovl), 142.9, 127.1, 19.9.

${ }^{1} \mathrm{H}$ NMR spectrum was consistent with that reported in the literature, while ${ }^{13} \mathrm{C}$ NMR spectrum was moderately consistent. ${ }^{[21]}$<smiles>CCc1ccncc1[N+](=O)[O-]</smiles>

4-ethyl-3-nitropyridine, $\mathbf{4 b}$

Yield:

$83 \mathrm{mg}(0.55 \mathrm{mmol}, \mathbf{2 8 \%})$ from $\mathbf{1 a}(248 \mathrm{mg}, 2.00 \mathrm{mmol})$ and $\mathbf{2 b}$ $107 \mathrm{mg}(0.70 \mathrm{mmol}, \mathbf{3 5 \%})$ from $\mathbf{1 a}(251 \mathrm{mg}, 2.02 \mathrm{mmol})$ and $\mathbf{2 b}$ at $\mathbf{- 4 0}{ }^{\circ} \mathbf{C}$, 3 min

pale yellow oil

eluent: toluene/ethyl acetate 50:1 to 2:1

${ }^{1}$ H NMR (400 MHz, CDCl $): \delta=9.05(\mathrm{~s}, 1 \mathrm{H}), 8.65(\mathrm{~d}, J=5.1 \mathrm{~Hz}, 1 \mathrm{H})$, $7.31(\mathrm{~d}, J=5.1 \mathrm{~Hz}, 1 \mathrm{H}), 2.94(\mathrm{q}, J=7.5 \mathrm{~Hz}, 2 \mathrm{H}), 1.28(\mathrm{t}, J=7.5 \mathrm{~Hz}, 3 \mathrm{H})$.

${ }^{13}$ C NMR (100 MHz, $\left.\mathbf{C D C l}_{3}\right): \delta=152.9,148.0,145.8,145.6,125.2,25.5$, 13.7 .

${ }^{1} \mathrm{H}$ NMR, ${ }^{13} \mathrm{C}$ NMR spectra were consistent with those reported in the literature. ${ }^{[21]}$ 
<smiles>CC(C)Cc1ccncc1[N+](=O)[O-]</smiles>

4-isobutyl-3-nitropyridine, $\mathbf{4 c}$

Yield:

$257 \mathrm{mg}(1.43 \mathrm{mmol}, \mathbf{7 1 \%})$ from $1 \mathbf{a}(250 \mathrm{mg}, 2.01 \mathrm{mmol})$ and $\mathbf{2 c}$

$678 \mathrm{mg}(3.76 \mathrm{mmol}, \mathbf{7 3 \%})$ from $\mathbf{1 a}(636 \mathrm{mg}, 5.13 \mathrm{mmol})$ and $\mathbf{3 c}$

orange oil

eluent: toluene/ethyl acetate 50:1 to 4:1

${ }^{1} \mathrm{H}$ NMR (400 MHz, CDCl $): \delta=9.00(\mathrm{~s}, 1 \mathrm{H}), 8.59(\mathrm{~d}, J=5.1 \mathrm{~Hz}, 1 \mathrm{H})$, $7.20(\mathrm{~d}, J=5.1 \mathrm{~Hz}, 1 \mathrm{H}), 2.76(\mathrm{~d}, J=7.2 \mathrm{~Hz}, 2 \mathrm{H}), 1.95-1.80(\mathrm{~m}, 1 \mathrm{H})$, $0.86(\mathrm{~d}, J=6.7 \mathrm{~Hz}, 6 \mathrm{H})$.

MS (EI): m/z (\%): $181\left(4,[\mathrm{M}+\mathrm{H}]^{+}\right), 163\left(15,[\mathrm{M}-\mathrm{OH}]^{+}\right), 138(37$, $\left.\left[\mathrm{M}-\mathrm{C}_{3} \mathrm{H}_{6}\right]^{+}\right), 121\left(100,\left[\mathrm{M}-\mathrm{OC}_{3} \mathrm{H}_{7}\right]^{+}\right), 44\left(86,\left[\mathrm{C}_{3} \mathrm{H}_{8}\right]^{+}\right)$.

HRMS (EI): m/z [M] ${ }^{+}$calcd for $\mathrm{C}_{9} \mathrm{H}_{12} \mathrm{~N}_{2} \mathrm{O}_{2}: 180.0899$; found: 180.0892 .<smiles>CC(C)Cc1ccc([N+](=O)[O-])cn1</smiles>

2-isobutyl-5-nitropyridine, $\mathbf{4} \mathbf{c}^{\prime}$

Yield:

$38 \mathrm{mg}(0.21 \mathrm{mmol}, \mathbf{1 0 \%})$ from $1 \mathrm{a}(250 \mathrm{mg}, 2.01 \mathrm{mmol})$ and $\mathbf{2 c}$

$79 \mathrm{mg}(0.44 \mathrm{mmol}, \mathbf{9 \%})$ from $1 \mathbf{a}(636 \mathrm{mg}, 5.13 \mathrm{mmol})$ and $\mathbf{3 c}$

pale yellow oil

eluent: toluene/ethyl acetate $50: 1$ to $4: 1$

${ }^{1} \mathbf{H}$ NMR $\left(400 \mathrm{MHz}, \mathbf{C D C l}_{3}\right): \delta=9.33(\mathrm{~d}, J=2.5 \mathrm{~Hz}, 1 \mathrm{H}), 8.35(\mathrm{dd}, J=$ $8.6,2.6 \mathrm{~Hz}, 1 \mathrm{H}), 7.28(\mathrm{~d}, J=8.6 \mathrm{~Hz}, 1 \mathrm{H}), 2.76(\mathrm{~d}, J=7.2 \mathrm{~Hz}, 2 \mathrm{H}), 2.20-$ $2.06(\mathrm{~m}, 1 \mathrm{H}), 0.92(\mathrm{~d}, J=6.7 \mathrm{~Hz}, 6 \mathrm{H})$.

${ }^{13}$ C NMR (100 MHz, $\left.\mathbf{C D C l}_{3}\right): \delta=168.5,144.7,142.5,131.0,123.5,47.5$, 29.2, 22.3.

MS (EI): m/z (\%): $181\left(11,[\mathrm{M}+\mathrm{H}]^{+}\right), 180\left(4,[\mathrm{M}]^{+}\right), 165\left(39,\left[\mathrm{M}-\mathrm{CH}_{3}\right]^{+}\right)$, $138\left(100,\left[\mathrm{M}-\mathrm{C}_{3} \mathrm{H}_{6}\right]^{+}\right)$.

HRMS (EI): $\mathrm{m} / \mathrm{z}[\mathrm{M}+\mathrm{H}]^{+}$calcd for $\mathrm{C}_{9} \mathrm{H}_{13} \mathrm{~N}_{2} \mathrm{O}_{2}:$ 181.0977; found: 181.0972 . 
<smiles>CC(C)(C)Cc1ccncc1[N+](=O)[O-]</smiles>
4-(tert-butylmethyl)-3-nitropyridine, $\mathbf{4 d}$

Yield:

$276 \mathrm{mg}(1.42 \mathrm{mmol}, \mathbf{7 0} \%)$ from $\mathbf{1 a}(253 \mathrm{mg}, 2.04 \mathrm{mmol})$

yellow oil

eluent: toluene/ethyl acetate 50:1 to 4:1

${ }^{1} \mathbf{H}$ NMR (400 MHz, CDCl $): \delta=8.91(\mathrm{~s}, 1 \mathrm{H}), 8.56(\mathrm{~d}, J=5.1 \mathrm{~Hz}, 1 \mathrm{H})$, $7.14(\mathrm{~d}, J=5.1 \mathrm{~Hz}, 1 \mathrm{H}), 2.90(\mathrm{~s}, 2 \mathrm{H}), 0.79(\mathrm{~s}, 9 \mathrm{H})$.

${ }^{13} \mathbf{C}$ NMR (100 MHz, $\left.\mathbf{C D C l}_{3}\right): \delta=151.6,147.3,145.3,142.8,127.7,43.7$, 33.1, 28.9.

MS (EI): m/z (\%): $195\left(2,[\mathrm{M}+\mathrm{H}]^{+}\right), 179\left(9,\left[\mathrm{M}-\mathrm{CH}_{3}\right]^{+}\right), 138$ (37, $\left.\left[\mathrm{M}-\mathrm{C}_{4} \mathrm{H}_{8}\right]^{+}\right), 121\left(22,\left[\mathrm{M}-\mathrm{OC}_{4} \mathrm{H}_{9}\right]^{+}\right), 57\left(100,\left[\mathrm{C}_{4} \mathrm{H}_{9}\right]^{+}\right)$.

HRMS (EI): $\mathrm{m} / \mathrm{z}[\mathrm{M}+\mathrm{H}]^{+}$calcd for $\mathrm{C}_{10} \mathrm{H}_{15} \mathrm{~N}_{2} \mathrm{O}_{2}$ : 195.1134; found: 195.1138 . 
<smiles>CCCCCCCc1ccncc1[N+](=O)[O-]</smiles>

4-octyl-3-nitropyridine, $\mathbf{4 e}$

Yield:

$335 \mathrm{mg}(1.42 \mathrm{mmol}, \mathbf{7 1 \%})$ from $\mathbf{1 a}(248 \mathrm{mg}, 2.00 \mathrm{mmol})$ and $\mathbf{3 e}$

$256 \mathrm{mg}(1.08 \mathrm{mmol}, \mathbf{5 3 \%})$ from $\mathbf{1 a}(251 \mathrm{mg}, 2.02 \mathrm{mmol})$ and $\mathbf{2 e}$

red liquid

eluent: toluene, then toluene/ethyl acetate $100: 1$ to $3: 1$

${ }^{1} \mathrm{H}$ NMR (400 MHz, $\left.\mathbf{C D C l}_{3}\right): \delta=9.02(\mathrm{~s}, 1 \mathrm{H}), 8.60(\mathrm{~d}, J=5.1 \mathrm{~Hz}, 1 \mathrm{H})$, $7.26(\mathrm{~d}, J=5.1,1 \mathrm{H}), 2.89-2.83(\mathrm{~m}, 2 \mathrm{H}), 1.64-1.54(\mathrm{~m}, 2 \mathrm{H}), 1.38-1.13$ $(\mathrm{m}, 10 \mathrm{H}), 0.81(\mathrm{t}, J=6.9 \mathrm{~Hz}, 3 \mathrm{H})$.

${ }^{13}$ C NMR (100 MHz, $\left.\mathbf{C D C l}_{3}\right): \delta=152.7,146.7,145.8,145.7,125.8,32.2$, 31.7, 29.7, 29.4, 29.1, 29.0, 22.5, 14.0.

MS (EI): m/z (\%): $237\left(2,[\mathrm{M}+\mathrm{H}]^{+}\right), 236\left(3,[\mathrm{M}]^{+}\right), 219\left(89,[\mathrm{M}-\mathrm{OH}]^{+}\right), 44$ $\left(100,\left[\mathrm{C}_{3} \mathrm{H}_{8}\right]^{+}\right)$.

HRMS (EI): $\mathrm{m} / \mathrm{z}$ [M] ${ }^{+}$calcd for $\mathrm{C}_{13} \mathrm{H}_{20} \mathrm{~N}_{2} \mathrm{O}_{2}$ : 236.1525; found: 236.1530 .<smiles>CCCCCCCc1ccc([N+](=O)[O-])cc1</smiles>

2-octyl-5-nitropyridine, $4 \mathrm{e}^{\prime}$

\section{Yield:}

$43 \mathrm{mg}(0.18 \mathrm{mmol}, \mathbf{9 \%})$ from $\mathbf{1 a}(248 \mathrm{mg}, 2.00 \mathrm{mmol})$ and $\mathbf{3 e}$

$51 \mathrm{mg}(0.21 \mathrm{mmol}, \mathbf{1 0 \%})$ from $1 \mathbf{a}(251 \mathrm{mg}, 2.02 \mathrm{mmol})$ and $\mathbf{2 e}$

yellowish oil

eluent: toluene, then toluene/ethyl acetate $100: 1$ to $3: 1$

${ }^{1} \mathbf{H}$ NMR $\left(400 \mathrm{MHz}, \mathbf{C D C l}_{3}\right): \delta=9.32(\mathrm{~d}, J=2.5 \mathrm{~Hz}, 1 \mathrm{H}), 8.35(\mathrm{dd}, J=$ 8.6, 2.7 Hz, 1H), $7.31(\mathrm{~d}, J=8.5 \mathrm{~Hz}, 1 \mathrm{H}), 2.92-2.86(\mathrm{~m}, 2 \mathrm{H}), 1.78-1.69$ $(\mathrm{m}, 2 \mathrm{H}), 1.38-1.16(\mathrm{~m}, 10 \mathrm{H}), 0.85(\mathrm{t}, J=6.9 \mathrm{~Hz}, 3 \mathrm{H})$.

${ }^{13}$ C NMR (100 MHz, $\left.\mathbf{C D C l}_{3}\right): \delta=169.4,144.6,142.5,131.3,122.8,38.5$, $31.8,29.5,29.32,29.25,29.1,22.6,14.1$.

MS (EI): m/z (\%): $237\left(1,[\mathrm{M}+\mathrm{H}]^{+}\right), 236\left(4,[\mathrm{M}]^{+}\right), 165\left(33,\left[\mathrm{M}-\mathrm{C}_{5} \mathrm{H}_{11}\right]^{+}\right)$, $151\left(49,\left[\mathrm{M}-\mathrm{C}_{6} \mathrm{H}_{13}\right]^{+}\right), 138\left(100,\left[\mathrm{M}-\mathrm{C}_{7} \mathrm{H}_{14}\right]^{+}\right)$,

HRMS (EI): $\mathrm{m} / \mathrm{z}$ [M] ${ }^{+}$calcd for $\mathrm{C}_{13} \mathrm{H}_{20} \mathrm{~N}_{2} \mathrm{O}_{2}$ : 236.1525; found: 236.1527 . 
<smiles>O=[N+]([O-])c1cnccc1CC1CC1</smiles>

4-(cyclopropylmethyl)-3-nitropyridine, $\mathbf{4 f}$

Yield:

$258 \mathrm{mg}(1.45 \mathrm{mmol}, \mathbf{7 2 \%})$ from $\mathbf{1 a}(248 \mathrm{mg}, 2.00 \mathrm{mmol})$

light orange oil

eluent: toluene/ethyl acetate 50:1 to 1:1

${ }^{1}$ H NMR (400 MHz, CDCl $): \delta=9.00(\mathrm{~s}, 1 \mathrm{H}), 8.63(\mathrm{~d}, J=5.1 \mathrm{~Hz}, 1 \mathrm{H})$, $7.50(\mathrm{~d}, J=5.1,1 \mathrm{H}), 2.81(\mathrm{~d}, J=7.0 \mathrm{~Hz}, 2 \mathrm{H}), 1.03-0.92(\mathrm{~m}, 1 \mathrm{H}), 0.58-$ $0.52(\mathrm{~m}, 2 \mathrm{H}), 0.21-0.15(\mathrm{~m}, 2 \mathrm{H})$.

${ }^{13} \mathbf{C}$ NMR (100 MHz, $\left.\mathbf{C D C l}_{3}\right): \delta=152.9,146.1,145.6,145.4,125.1,36.1$, 9.5, 4.8.

MS (EI): m/z (\%): $179\left(65,[\mathrm{M}+\mathrm{H}]^{+}\right), 161\left(19,[\mathrm{M}-\mathrm{OH}]^{+}\right), 135(100$, $\left.\left[\mathrm{M}-\mathrm{C}_{3} \mathrm{H}_{7}\right]^{+}\right)$.

HRMS (EI): $\mathrm{m} / \mathrm{z}[\mathrm{M}+\mathrm{H}]^{+}$calcd for $\mathrm{C}_{9} \mathrm{H}_{11} \mathrm{~N}_{2} \mathrm{O}_{2}:$ 179.0821; found: 179.0820 .<smiles>O=[N+]([O-])c1ccc(CC2CC2)nc1</smiles>

2-(cyclopropylmethyl)-5-nitropyridine, 4f'

Yield:

$32 \mathrm{mg}(0.18 \mathrm{mmol}, \mathbf{9 \%})$ form $\mathbf{1 a}(248 \mathrm{mg}, 2.00 \mathrm{mmol})$

yellowish oil

eluent: toluene/ethyl acetate 50:1 to 1:1

${ }^{1}$ H NMR (400 MHz, $\left.\mathbf{C D C l}_{3}\right): \delta=9.33(\mathrm{~d}, J=2.6 \mathrm{~Hz}, 1 \mathrm{H}), 8.38(\mathrm{dd}, J=$ 8.6, $2.7 \mathrm{~Hz}, 1 \mathrm{H}), 7.47(\mathrm{~d}, J=8.5 \mathrm{~Hz}, 1 \mathrm{H}), 2.82(\mathrm{~d}, J=7.1 \mathrm{~Hz}, 2 \mathrm{H}), 1.15-$ $1.04(\mathrm{~m}, 1 \mathrm{H}), 0.62-0.56(\mathrm{~m}, 2 \mathrm{H}), 0.28-0.23(\mathrm{~m}, 2 \mathrm{H})$.

${ }^{13}$ C NMR (100 MHz, $\left.\mathbf{C D C l}_{3}\right): \delta=168.8,144.6,142.6,131.3,122.4,42.9$, 10.2, 4.8.

MS (EI): m/z (\%): $179\left(9,[\mathrm{M}+\mathrm{H}]^{+}\right), 178\left(48,[\mathrm{M}]^{+}\right), 177\left(100,[\mathrm{M}-\mathrm{H}]^{+}\right)$, $163\left(68,\left[\mathrm{M}-\mathrm{CH}_{3}\right]^{+}\right), 131\left(57,\left[\mathrm{M}-\mathrm{HNO}_{2}\right]^{+}\right)$.

HRMS (EI): $\mathrm{m} / \mathrm{z}[\mathrm{M}+\mathrm{H}]^{+}$calcd for $\mathrm{C}_{9} \mathrm{H}_{11} \mathrm{~N}_{2} \mathrm{O}_{2}$ : 179.0821; found: 179.0814 . 
<smiles>O=[N+]([O-])c1cnccc1CCC1CC1(Cl)Cl</smiles><smiles>CN1CCC(Cc2ccncc2[N+](=O)[O-])CC1</smiles>

4-(2-(2,2-dichlorocyclopropyl)ethyl)-3-nitropyridine, $\mathbf{4 g}$

\section{Yield:}

$336 \mathrm{mg}(1.29 \mathrm{mmol}, \mathbf{6 4 \%})$ from $1 \mathrm{a}(250 \mathrm{mg}, 2.01 \mathrm{mmol})$

brown oil

eluent: toluene/ethyl acetate 20:1 to 5:1

${ }^{1}$ H NMR (400 MHz, CDCl $): \delta=9.09(\mathrm{~s}, 1 \mathrm{H}), 8.66(\mathrm{~d}, J=5.1 \mathrm{~Hz}, 1 \mathrm{H})$, $7.32(\mathrm{~d}, J=5.1 \mathrm{~Hz}, 1 \mathrm{H}), 3.19-3.01(\mathrm{~m}, 2 \mathrm{H}), 2.00-1.79(\mathrm{~m}, 3 \mathrm{H}), 1.63-$ $1.50(\mathrm{~m}, 2 \mathrm{H}), 1.12-1.03(\mathrm{~m}, 1 \mathrm{H})$.

${ }^{13}$ C NMR (100 MHz, $\left.\mathbf{C D C l}_{3}\right): \delta=153.1,146.0,145.6,145.1,126.2,60.7$, $31.4,30.5,29.9,26.5$.

MS (EI): $\mathrm{m} / \mathrm{z}(\%)=265,263,261\left(1,5,7,[\mathrm{M}]^{+}\right), 121,119(31,100$, $\left.\left[\mathrm{OC}_{5} \mathrm{H}_{8} \mathrm{Cl}\right]^{+}\right)$.

HRMS (EI): $\mathrm{m} / \mathrm{z}[\mathrm{M}]^{+}$calcd for $\mathrm{C}_{10} \mathrm{H}_{11} \mathrm{O}_{2} \mathrm{~N}_{2}{ }^{35} \mathrm{Cl}_{2}$ : 261.0198; found: 261.0192 .

4-((1-methyl-4-piperidinyl)methyl)-3-nitropyridine, $\mathbf{4 h}$

Yield:

$290 \mathrm{mg}$ (1.23 mmol, 61\%) from 1a (252 mg, $2.03 \mathrm{mmol})$

orange oil

eluent: ethyl acetate then ethyl acetate/MeOH 50:1 to 10:1 (all with 2\% v/v $\mathrm{NEt}_{3}$ )

${ }^{1} \mathrm{H}$ NMR (400 MHz, CDCl $): \delta=9.11(\mathrm{~s}, 1 \mathrm{H}), 8.65(\mathrm{~d}, J=5.0 \mathrm{~Hz}, 1 \mathrm{H})$, $7.22(\mathrm{~d}, J=5.0 \mathrm{~Hz}, 1 \mathrm{H}), 3.07(\mathrm{~d}, J=12.3 \mathrm{~Hz}, 2 \mathrm{H}), 2.89(\mathrm{~d}, J=5.6 \mathrm{~Hz}$, $2 \mathrm{H}), 2.42(\mathrm{~s}, 3 \mathrm{H}), 2.23-2.13(\mathrm{~m}, 2 \mathrm{H}), 1.71-1.63(\mathrm{~m}, 5 \mathrm{H})$.

${ }^{13}$ C NMR (100 MHz, $\left.\mathbf{C D C l}_{3}\right): \delta=152.9,146.3,145.7,144.0,127.1,54.9$, 45.1, 39.0, 34.9, 30.7 .

MS (EI): m/z (\%): $235\left(27,[\mathrm{M}]^{+}\right), 234\left(55,[\mathrm{M}-\mathrm{H}]^{+}\right), 218\left(48,[\mathrm{M}-\mathrm{OH}]^{+}\right)$, $98\left(87,\left[\mathrm{C}_{6} \mathrm{H}_{12} \mathrm{~N}\right]^{+}\right), 96\left(100,\left[\mathrm{C}_{6} \mathrm{H}_{10} \mathrm{~N}\right]^{+}\right)$.

HRMS (EI): $\mathrm{m} / \mathrm{z}$ [M] ${ }^{+}$calcd for $\mathrm{C}_{12} \mathrm{H}_{17} \mathrm{~N}_{3} \mathrm{O}_{2}$ : 235.1321; found: 235.1313 . 
<smiles>COc1ccc([N+](=O)[O-])c(C)n1</smiles><smiles>CCCCCCCCc1nc(OC)ccc1[N+](=O)[O-]</smiles>

6-methoxy-2-methyl-3-nitropyridine, $\mathbf{4 i}$

Yield:

$183 \mathrm{mg}$ (1.09 mmol, 54\%) from $\mathbf{1 b}(309 \mathrm{mg}, 2.00 \mathrm{mmol})$

white crystals, mp $67.5-68.5^{\circ} \mathrm{C}$

eluent: cyclohexane/toluene $3: 1$ to $1: 1$, then toluene, then toluene/ethyl acetate $20: 1$ to $5: 1$

${ }^{1}$ H NMR (400 MHz, CDCl $): \delta=8.19(\mathrm{~d}, J=9.0 \mathrm{~Hz}, 1 \mathrm{H}), 6.60(\mathrm{~d}, J=$ $9.0 \mathrm{~Hz}, 1 \mathrm{H}), 3.95$ (s, 3H), 2.74 (s, 3H).

${ }^{13} \mathbf{C}$ NMR (100 MHz, $\left.\mathbf{C D C l}_{3}\right): \delta=164.7,154.5,139.6,135.8,108.9,54.3$, 24.5.

MS (EI): m/z (\%): $169\left(22,[\mathrm{M}+\mathrm{H}]^{+}\right), 168\left(100,[\mathrm{M}]^{+}\right), 167\left(90,[\mathrm{M}-\mathrm{H}]^{+}\right)$, $151\left(73,[\mathrm{M}-\mathrm{OH}]^{+}\right), 138\left(50,\left[\mathrm{M}-\mathrm{OCH}_{2}\right]^{+}\right), 107\left(87,\left[\mathrm{M}-\mathrm{CH}_{3} \mathrm{NO}_{2}\right]^{+}\right)$.

HRMS (EI): $\mathrm{m} / \mathrm{z}[\mathrm{M}]^{+}$calcd for $\mathrm{C}_{7} \mathrm{H}_{8} \mathrm{~N}_{2} \mathrm{O}_{3}: 168.0535$; found: 168.0533 .

6-methoxy-2-octyl-3-nitropyridine, $\mathbf{4 j}$

\section{Yield:}

$295 \mathrm{mg}$ (1.11 mmol, 55\%) from $\mathbf{1 b}(309 \mathrm{mg}, 2.01 \mathrm{mmol})$

white crystals, $\mathbf{m p} 56.5-57.5^{\circ} \mathrm{C}$

eluent: cyclohexane/toluene $5: 1$ to $1: 1$, then toluene, then toluene/ethyl acetate $20: 1$ to $5: 1$

${ }^{1}$ H NMR (400 MHz, CDCl $\left.\mathbf{~}_{3}\right): \delta=8.15(\mathrm{~d}, J=8.9 \mathrm{~Hz}, 1 \mathrm{H}), 6.59(\mathrm{~d}, J=$ $8.9 \mathrm{~Hz}, 1 \mathrm{H}), 3.97(\mathrm{~s}, 3 \mathrm{H}), 3.07-3.01(\mathrm{~m}, 2 \mathrm{H}), 1.78-1.68(\mathrm{~m}, 2 \mathrm{H}), 1.41-$ $1.17(\mathrm{~m}, 10 \mathrm{H}), 0.83(\mathrm{t}, J=6.9 \mathrm{~Hz}, 3 \mathrm{H})$.

${ }^{13}$ C NMR (100 MHz, $\left.\mathbf{C D C l}_{3}\right): \delta=164.7,157.9,139.8,135.9,108.6,54.2$, $35.9,31.8,29.5,29.3,29.2,28.2,22.6,14.0$.

MS (EI): m/z (\%): $266\left(1,[\mathrm{M}]^{+}\right), 265\left(2,[\mathrm{M}-\mathrm{H}]^{+}\right), 249\left(15,[\mathrm{M}-\mathrm{OH}]^{+}\right), 195$ $\left(69,\left[\mathrm{M}-\mathrm{C}_{5} \mathrm{H}_{11}\right]^{+}\right), 168\left(100,\left[\mathrm{M}-\mathrm{C}_{7} \mathrm{H}_{14}\right]^{+}\right)$,

HRMS (EI): $\mathrm{m} / \mathrm{z}$ [M] ${ }^{+}$calcd for $\mathrm{C}_{14} \mathrm{H}_{22} \mathrm{~N}_{2} \mathrm{O}_{3}$ : 266.1630; found: 266.1619 . 
<smiles>CCCCCCCc1cc(OC)c([N+](=O)[O-])cn1</smiles>

4-methoxy-2-octyl-5-nitropyridine, 4k

Yield:

$211 \mathrm{mg}(0.79 \mathrm{mmol}, \mathbf{4 0 \%})$ from $1 \mathrm{c}(309 \mathrm{mg}, 2.00 \mathrm{mmol})$

light brown oil

eluent: cyclohexane/toluene $5: 1$ to $1: 1$, then toluene, then toluene/ethyl acetate $5: 1$

${ }^{1} \mathrm{H}$ NMR (400 MHz, $\left.\mathbf{C D C l}_{3}\right): \delta=8.88(\mathrm{~s}, 1 \mathrm{H}), 6.80(\mathrm{~s}, 1 \mathrm{H}), 3.96(\mathrm{~s}, 3 \mathrm{H})$, $2.78-2.72(\mathrm{~m}, 2 \mathrm{H}), 1.72-1.62(\mathrm{~m}, 2 \mathrm{H}), 1.35-1.13(\mathrm{~m}, 10 \mathrm{H}), 0.80(\mathrm{t}$, $J=6.9 \mathrm{~Hz}, 3 \mathrm{H})$.

${ }^{13}$ C NMR (100 MHz, $\left.\mathbf{C D C l}_{3}\right): \delta=169.6,159.1,146.7,134.5,106.8,56.4$, $38.8,31.7,29.4,29.23,29.19,29.0,22.5,14.0$.

MS (EI): m/z (\%): $267\left(3,[\mathrm{M}+\mathrm{H}]^{+}\right), 266\left(5,[\mathrm{M}]^{+}\right), 265\left(3,[\mathrm{M}-\mathrm{H}]^{+}\right), 181$ $\left(39,\left[\mathrm{M}-\mathrm{C}_{6} \mathrm{H}_{13}\right]^{+}\right), 168\left(100,\left[\mathrm{M}-\mathrm{C}_{7} \mathrm{H}_{14}\right]^{+}\right)$.

HRMS (EI): $\mathrm{m} / \mathrm{z}$ [M] $]^{+}$calcd for $\mathrm{C}_{14} \mathrm{H}_{22} \mathrm{~N}_{2} \mathrm{O}_{3}: 266.1630$; found: 266.1631 .<smiles>CCCCCCCc1nccc(OC)c1[N+](=O)[O-]</smiles>

4-methoxy-2-octyl-3-nitropyridine, 4k'

\section{Yield:}

$73 \mathrm{mg}(0.28 \mathrm{mmol}, \mathbf{1 4 \%})$ from $1 \mathrm{c}(309 \mathrm{mg}, 2.00 \mathrm{mmol})$

dark orange oil

eluent: cyclohexane/toluene $5: 1$ to $1: 1$, then toluene, then toluene/ethyl acetate $5: 1$

${ }^{1}$ H NMR (400 MHz, $\left.\mathbf{C D C l}_{3}\right): \delta=8.45(\mathrm{~d}, J=5.8 \mathrm{~Hz}, 1 \mathrm{H}), 6.80(\mathrm{~d}, J=$ $5.8 \mathrm{~Hz}, 1 \mathrm{H}), 3.91(\mathrm{~s}, J=2.2 \mathrm{~Hz}, 3 \mathrm{H}), 2.70-2.64(\mathrm{~m}, 2 \mathrm{H}), 1.74-1.63(\mathrm{~m}$, $2 \mathrm{H}), 1.34-1.12(\mathrm{~m}, 10 \mathrm{H}), 0.83(\mathrm{t}, J=6.9 \mathrm{~Hz}, 3 \mathrm{H})$.

${ }^{13} \mathbf{C}$ NMR (100 MHz, $\left.\mathbf{C D C l}_{3}\right): \delta=157.0,154.8,151.5,138.5,105.5,56.4$, $33.5,31.7,29.3,29.2,29.1,28.9,22.6,14.0$.

HRMS (ESI): $\mathrm{m} / \mathrm{z}[\mathrm{M}+\mathrm{H}]^{+}$calcd for $\mathrm{C}_{14} \mathrm{H}_{23} \mathrm{~N}_{2} \mathrm{O}_{3}:$ : 267.1709; found: 267.1708. 
<smiles>CC(C)Cc1cc(Sc2ccccc2)ncc1[N+](=O)[O-]</smiles>

2-thiophenoxy-4-isobutyl-5-nitropyridine, 41

Yield:

$405 \mathrm{mg}$ (1.40 mmol, 69\%) from 1d (467 mg, $2.01 \mathrm{mmol})$

orange solid, mp $47.0-50.0{ }^{\circ} \mathrm{C}$

eluent: cyclohexane/toluene $5: 1$ to $1: 1$, then toluene, then toluene/ethyl acetate $20: 1$ to $5: 1$

${ }^{1} \mathbf{H}$ NMR (400 MHz, $\left.\mathbf{C D C l}_{3}\right): \delta=8.91(\mathrm{~s}, 1 \mathrm{H}), 7.59-7.53(\mathrm{~m}, 2 \mathrm{H}), 7.48$ $-7.41(\mathrm{~m}, 3 \mathrm{H}), 6.65(\mathrm{~s}, 1 \mathrm{H}), 2.65(\mathrm{~d}, J=7.1 \mathrm{~Hz}, 2 \mathrm{H}), 1.84-1.70(\mathrm{~m}, 1 \mathrm{H})$, $0.81(\mathrm{~d}, J=6.7 \mathrm{~Hz}, 6 \mathrm{H})$.

${ }^{13}$ C NMR (100 MHz, $\left.\mathbf{C D C l}_{3}\right): \delta=167.2,146.2,146.0,142.6,135.2,130.0$, $129.8,128.8,122.4,41.3,28.7,22.1$.

MS (EI): m/z (\%): $289\left(22,[\mathrm{M}+\mathrm{H}]^{+}\right), 288\left(56,[\mathrm{M}]^{+}\right), 287\left(100,[\mathrm{M}-\mathrm{H}]^{+}\right)$, $199\left(40,\left[\mathrm{M}-\mathrm{C}_{3} \mathrm{H}_{7} \mathrm{NO}_{2}\right]^{+}\right)$.

HRMS (EI): $\mathrm{m} / \mathrm{z}$ [M] ${ }^{+}$calcd for $\mathrm{C}_{15} \mathrm{H}_{16} \mathrm{~N}_{2} \mathrm{O}_{2} \mathrm{~S}: 288.0932$; found: 288.0922 . 
<smiles>CC(C)(C)Cc1cc(Sc2ccccc2)ncc1[N+](=O)[O-]</smiles><smiles>CC(C)(C)Cc1nc(S)ccc1[N+](=O)[O-]</smiles>

2-thiophenoxy-4-(tert-butylmethyl)-5-nitropyridine, $\mathbf{4 m}$

Yield:

$526 \mathrm{mg}$ (1.73 mmol, 86\%) from $\mathbf{1 d}(469 \mathrm{mg}, 2.02 \mathrm{mmol})$

pale yellow solid, mp $60.0-62.5^{\circ} \mathrm{C}$

eluent: cyclohexane/toluene $3: 1$ to $1: 1$, then toluene, then toluene/ethyl acetate $5: 1$

${ }^{1} \mathbf{H}$ NMR (400 MHz, $\left.\mathbf{C D C l}_{3}\right): \delta=8.81(\mathrm{~s}, 1 \mathrm{H}), 7.59-7.52(\mathrm{~m}, 2 \mathrm{H}), 7.47$ $-7.39(\mathrm{~m}, 3 \mathrm{H}), 6.55(\mathrm{~s}, 1 \mathrm{H}), 2.79(\mathrm{~s}, 2 \mathrm{H}), 0.73(\mathrm{~s}, 9 \mathrm{H})$.

${ }^{13}$ C NMR (100 MHz, $\left.\mathbf{C D C l}_{3}\right): \delta=166.4,145.6,143.9,143.5,135.3,130.0$, $129.8,128.8,123.4,43.8,33.2,28.9$.

MS (EI): m/z (\%): $303\left(33,[\mathrm{M}+\mathrm{H}]^{+}\right), 302\left(76,[\mathrm{M}]^{+}\right), 301\left(100,[\mathrm{M}-\mathrm{H}]^{+}\right)$, $287\left(26,\left[\mathrm{M}-\mathrm{CH}_{3}\right]^{+}\right), 245\left(28,\left[\mathrm{M}-\mathrm{C}_{4} \mathrm{H}_{9}\right]^{+}\right), 229\left(37,\left[\mathrm{M}-\mathrm{OC}_{4} \mathrm{H}_{9}\right]^{+}\right), 199(25$, $\left.\left[\mathrm{M}-\mathrm{C}_{4} \mathrm{H}_{9} \mathrm{NO}_{2}\right]^{+}\right), 58\left(70,\left[\mathrm{C}_{4} \mathrm{H}_{10}\right]^{+}\right)$.

HRMS (EI): $\mathrm{m} / \mathrm{z}$ [M] ${ }^{+}$calcd for $\mathrm{C}_{16} \mathrm{H}_{18} \mathrm{~N}_{2} \mathrm{O}_{2} \mathrm{~S}: 302.1089$; found: 302.1089 .

6-thiophenoxy-2-(tert-butylmethyl)-3-nitropyridine, $4 \mathbf{m}$ '

Yield:

$28 \mathrm{mg}(0.09 \mathrm{mmol}, \mathbf{5 \%})$ from $1 \mathrm{~d}(469 \mathrm{mg}, 2.02 \mathrm{mmol})$

yellow oil

eluent: cyclohexane/toluene $3: 1$ to $1: 1$, then toluene, then toluene/ethyl acetate $5: 1$

${ }^{1} \mathbf{H}$ NMR $\left(400 \mathrm{MHz}, \mathbf{C D C l}_{3}\right): \delta=7.90(\mathrm{~d}, J=8.7 \mathrm{~Hz}, 1 \mathrm{H}), 7.62-7.56(\mathrm{~m}$, 2H), $7.49-7.42(\mathrm{~m}, 3 \mathrm{H}), 6.76(\mathrm{~d}, J=8.7 \mathrm{~Hz}, 1 \mathrm{H}), 3.09$ (s, 2H), 0.85 (s, $9 \mathrm{H})$.

${ }^{13}$ C NMR (100 MHz, CDCl $\left.\mathbf{3}\right): \delta=165.9,155.1,143.9,135.8,132.8,130.0$, $129.9,128.9,117.8,46.3,33.6,29.5$.

MS (EI): m/z (\%): $303\left(14,[\mathrm{M}+\mathrm{H}]^{+}\right), 302\left(26,[\mathrm{M}]^{+}\right), 287\left(39,\left[\mathrm{M}-\mathrm{CH}_{3}\right]^{+}\right)$, $272\left(84,\left[\mathrm{M}-\mathrm{CH}_{3}-\mathrm{CH}_{3}\right]^{+}\right), 246\left(94,\left[\mathrm{M}-\mathrm{C}_{4} \mathrm{H}_{8}\right]^{+}\right), 229\left(53,\left[\mathrm{M}-\mathrm{OC}_{4} \mathrm{H}_{9}\right]^{+}\right), 57$ $\left(100,\left[\mathrm{C}_{4} \mathrm{H}_{9}\right]^{+}\right)$.

HRMS (EI): $\mathrm{m} / \mathrm{z}$ [M] ${ }^{+}$calcd for $\mathrm{C}_{16} \mathrm{H}_{18} \mathrm{~N}_{2} \mathrm{O}_{2} \mathrm{~S}: 302.1089$; found: 302.1100 . 
<smiles>CCCCCCCCc1cc(Sc2ccccc2)ncc1[N+](=O)[O-]</smiles>

2-thiophenoxy-4-octyl-5-nitropyridine, 4 n

Yield:

$442 \mathrm{mg}$ (1.28 mmol, 64\%) from $1 \mathrm{~d}(467 \mathrm{mg}, 2.01 \mathrm{mmol})$

orange oil

eluent: cyclohexane/toluene $5: 1$ to $1: 1$, then toluene, then toluene/ethyl acetate $20: 1$ to $5: 1$

${ }^{1} \mathbf{H}$ NMR (400 MHz, $\left.\mathbf{C D C l}_{3}\right): \delta=8.93(\mathrm{~s}, 1 \mathrm{H}), 7.61-7.56(\mathrm{~m}, 2 \mathrm{H}), 7.51$ $-7.43(\mathrm{~m}, 3 \mathrm{H}), 6.73(\mathrm{~s}, 1 \mathrm{H}), 2.81-2.74(\mathrm{~m}, 2 \mathrm{H}), 1.52-1.42(\mathrm{~m}, 2 \mathrm{H}), 1.32$ $-1.17(\mathrm{~m}, 10 \mathrm{H}), 0.86(\mathrm{t}, J=7.0 \mathrm{~Hz}, 3 \mathrm{H})$.

${ }^{13}$ C NMR (100 MHz, $\left.\mathbf{C D C l}_{3}\right): \delta=167.5,147.5,146.3,142.5,135.4,130.1$, 129.9, 128.9, 121.7, 32.7, 31.7, 29.4, 29.3, 29.1, 29.0, 22.6, 14.1.

MS (EI): m/z (\%): $345\left(25,[\mathrm{M}+\mathrm{H}]^{+}\right), 344\left(73,[\mathrm{M}]^{+}\right), 343\left(100,[\mathrm{M}-\mathrm{H}]^{+}\right)$, $327\left(52,[\mathrm{M}-\mathrm{OH}]^{+}\right), 245\left(100,\left[\mathrm{M}-\mathrm{C}_{7} \mathrm{H}_{15}\right]^{+}\right), 199\left(69,\left[\mathrm{M}-\mathrm{C}_{7} \mathrm{H}_{15} \mathrm{NO}_{2}\right]^{+}\right)$.

HRMS (EI): $\mathrm{m} / \mathrm{z}[\mathrm{M}+\mathrm{H}]^{+}$calcd for $\mathrm{C}_{19} \mathrm{H}_{25} \mathrm{~N}_{2} \mathrm{O}_{2} \mathrm{~S}$ : 345.1637; found: 345.1638 .<smiles>CCCCCCCc1nc(Sc2ccccc2)ccc1[N+](=O)[O-]</smiles>

6-thiophenoxy-2-octyl-3-nitropyridine, 4 n'

Yield:

$41 \mathrm{mg}(0.12 \mathrm{mmol}, \mathbf{6 \%})$ from $1 \mathrm{~d}(467 \mathrm{mg}, 2.01 \mathrm{mmol})$

yellowish oil

eluent: cyclohexane/toluene $5: 1$ to $1: 1$, then toluene, then toluene/ethyl acetate $20: 1$ to $5: 1$

${ }^{1} \mathbf{H}$ NMR (400 MHz, $\left.\mathbf{C D C l}_{3}\right): \delta=7.98(\mathrm{~d}, J=8.7 \mathrm{~Hz}, 1 \mathrm{H}), 7.62-7.57(\mathrm{~m}$, $2 \mathrm{H}), 7.51-7.43(\mathrm{~m}, 3 \mathrm{H}), 6.70(\mathrm{~d}, J=8.7 \mathrm{~Hz}, 1 \mathrm{H}), 3.07-3.01(\mathrm{~m}, 2 \mathrm{H})$, $1.72-1.61(\mathrm{~m}, 2 \mathrm{H}), 1.40-1.19(\mathrm{~m}, 10 \mathrm{H}), 0.87(\mathrm{t}, J=6.9 \mathrm{~Hz}, 3 \mathrm{H})$.

${ }^{13}$ C NMR (100 MHz, CDCl $\left.\mathbf{3}\right): \delta=167.3,157.8,142.1,135.7,133.1,130.1$, 130.0, 128.9, 117.6, 36.0, 31.9, 29.5, 29.3, 29.2, 28.5, 22.7, 14.1.

MS (EI): m/z (\%): $345\left(10,[\mathrm{M}+\mathrm{H}]^{+}\right), 344\left(26,[\mathrm{M}]^{+}\right), 327\left(18,[\mathrm{M}-\mathrm{OH}]^{+}\right)$, $273\left(100,\left[\mathrm{M}-\mathrm{C}_{5} \mathrm{H}_{11}\right]^{+}\right), 229\left(72,\left[\mathrm{M}-\mathrm{OC}_{7} \mathrm{H}_{15}\right]^{+}\right)$.

HRMS (EI): $\mathrm{m} / \mathrm{z}$ [M] ${ }^{+}$calcd for $\mathrm{C}_{19} \mathrm{H}_{24} \mathrm{~N}_{2} \mathrm{O}_{2} \mathrm{~S}$ : 344.1559; found: 344.1563 . 
<smiles>O=[N+]([O-])c1cnc(Sc2ccccc2)cc1CC1CC1</smiles><smiles>O=[N+]([O-])c1ccc(Sc2ccccc2)nc1CC1CC1</smiles>

2-thiophenoxy-4-cyclopropylmethyl-5-nitropyridine, 40

Yield:

$417 \mathrm{mg}$ (1.46 mmol, 73\%) form $\mathbf{1 d}(466 \mathrm{mg}, 2.01 \mathrm{mmol})$

light red solid, $\mathbf{m p} 48.0-50.5^{\circ} \mathrm{C}$

eluent: cyclohexane/toluene $3: 1$ to $1: 1$, then toluene, then toluene/ethyl acetate $20: 1$ to $5: 1$

${ }^{1} \mathbf{H}$ NMR (400 MHz, $\left.\mathbf{C D C l}_{3}\right): \delta=8.90(\mathrm{~s}, 1 \mathrm{H}), 7.61-7.54(\mathrm{~m}, 2 \mathrm{H}), 7.48$ $-7.41(\mathrm{~m}, 3 \mathrm{H}), 7.00(\mathrm{~s}, 1 \mathrm{H}), 2.73(\mathrm{~d}, J=7.0 \mathrm{~Hz}, 2 \mathrm{H}), 0.80-0.69(\mathrm{~m}, 1 \mathrm{H})$, $0.41-0.35(\mathrm{~m}, 2 \mathrm{H}), 0.07-0.00(\mathrm{~m}, 2 \mathrm{H})$.

${ }^{13}$ C NMR (100 MHz, CDCl 3 ): $\delta=168.1,146.8,145.8,142.0,135.3,130.0$, $129.8,128.8,120.8,36.5,9.1,4.6$.

MS (EI): m/z (\%): $287\left(25,[\mathrm{M}+\mathrm{H}]^{+}\right), 286\left(73,[\mathrm{M}]^{+}\right), 285\left(100,[\mathrm{M}-\mathrm{H}]^{+}\right)$, $231\left(32,\left[\mathrm{M}-\mathrm{C}_{4} \mathrm{H}_{7}\right]^{+}\right)$.

HRMS (EI): $\mathrm{m} / \mathrm{z}$ [M] ${ }^{+}$calcd for $\mathrm{C}_{15} \mathrm{H}_{14} \mathrm{~N}_{2} \mathrm{O}_{2} \mathrm{~S}$ : 286.0776; found: 286.0772 .

6-thiophenoxy-2-cyclopropylmethyl-3-nitropyridine, $\mathbf{4 0}^{\prime}$

\section{Yield:}

$43 \mathrm{mg}(0.15 \mathrm{mmol}, \mathbf{8 \%})$ form $\mathbf{1 d}(466 \mathrm{mg}, 2.01 \mathrm{mmol})$

yellow oil

eluent: cyclohexane/toluene $3: 1$ to $1: 1$, then toluene, then toluene/ethyl acetate $20: 1$ to $5: 1$

${ }^{1} \mathrm{H}$ NMR $\left(400 \mathrm{MHz}, \mathbf{C D C l}_{3}\right): \delta=8.00(\mathrm{~d}, J=8.7 \mathrm{~Hz}, 1 \mathrm{H}), 7.63-7.56(\mathrm{~m}$, 2H), $7.50-7.42(\mathrm{~m}, 3 \mathrm{H}), 6.77(\mathrm{~d}, J=8.7 \mathrm{~Hz}, 1 \mathrm{H}), 2.96(\mathrm{~d}, J=7.0 \mathrm{~Hz}$, $2 \mathrm{H}), 1.20-1.08(\mathrm{~m}, 1 \mathrm{H}), 0.45-0.38(\mathrm{~m}, 2 \mathrm{H}), 0.24-0.18(\mathrm{~m}, 2 \mathrm{H})$.

${ }^{13}$ C NMR (100 MHz, CDCl $\left.\mathbf{3}\right): \delta=167.1,157.1,142.0,135.6,133.0,130.0$, $129.9,128.9,117.9,40.4,10.0,4.5$.

MS (EI): m/z (\%): $287\left(31,[\mathrm{M}+\mathrm{H}]^{+}\right), 286\left(100,[\mathrm{M}]^{+}\right), 271\left(69,\left[\mathrm{M}-\mathrm{CH}_{3}\right]^{+}\right)$, $209\left(83,\left[\mathrm{M}-\mathrm{C}_{6} \mathrm{H}_{5}\right]^{+}\right)$.

HRMS (EI): $\mathrm{m} / \mathrm{z}$ [M] ${ }^{+}$calcd for $\mathrm{C}_{15} \mathrm{H}_{14} \mathrm{~N}_{2} \mathrm{O}_{2} \mathrm{~S}: 286.0776$; found: 286.0777 . 
<smiles>O=[N+]([O-])c1cnc(S)cc1CCC1CC1(Cl)Cl</smiles><smiles>O=[N+]([O-])c1ccc(Sc2ccccc2)nc1CCC1CC1(Cl)Cl</smiles>

4-(2-(2,2-dichlorocyclopropyl)ethyl)-2-thiophenoxy-5-nitropyridine, 4p

\section{Yield:}

$394 \mathrm{mg}$ (1.07 mmol, 53\%) from $\mathbf{1 d}(467 \mathrm{mg}, 2.01 \mathrm{mmol})$

yellow oil

eluent: cyclohexane/toluene $3: 1$ to $1: 1$, then toluene, then toluene/ethyl acetate $20: 1$ to $5: 1$

${ }^{1} \mathbf{H}$ NMR (400 MHz, $\left.\mathbf{C D C l}_{3}\right): \delta=8.99(\mathrm{~s}, 1 \mathrm{H}), 7.61-7.56(\mathrm{~m}, 2 \mathrm{H}), 7.51$ $-7.43(\mathrm{~m}, 3 \mathrm{H}), 6.76(\mathrm{~s}, 1 \mathrm{H}), 3.09(\mathrm{ddd}, J=13.2,8.8,6.1 \mathrm{~Hz}, 1 \mathrm{H}), 2.89$ (ddd, $J=13.2,9.1,6.1 \mathrm{~Hz}, 1 \mathrm{H}), 1.94-1.81(\mathrm{~m}, 1 \mathrm{H}), 1.76-1.64(\mathrm{~m}, 1 \mathrm{H})$, $1.57-1.43(\mathrm{~m}, 2 \mathrm{H}), 1.06-1.01(\mathrm{~m}, 1 \mathrm{H})$.

${ }^{13}$ C NMR (100 MHz, CDCl $\left.\mathbf{3}\right): \delta=168.3,146.6,145.9,142.1,135.5,130.2$, 130.0, 128.6, 122.0, 60.8, 32.0, 30.4, 29.9, 26.6.

MS (EI): $\mathrm{m} / \mathrm{z}(\%)=372,370,368\left(14,65,87,[\mathrm{M}]^{+}\right), 371,369,367(28$, $\left.89,100,[\mathrm{M}-\mathrm{H}]^{+}\right), 335,333\left(41,91,[\mathrm{M}-\mathrm{Cl}]^{+}\right), 227\left(95,\left[\mathrm{M}-\mathrm{NO}_{2}-\mathrm{C}_{2} \mathrm{HCl}_{2}\right]^{+}\right)$.

HRMS (EI): $\mathrm{m} / \mathrm{z}$ [M] $]^{+}$calcd for $\mathrm{C}_{16} \mathrm{H}_{14} \mathrm{O}_{2} \mathrm{~N}_{2} \mathrm{~S}^{35} \mathrm{Cl}_{2}:$ 368.0153; found: 368.0150 .

2-(2-(2,2-dichlorocyclopropyl)ethyl)-6-thiophenoxy-3-nitropyridine, 4p'

\section{Yield:}

$67 \mathrm{mg}(0.18 \mathrm{mmol}, \mathbf{9 \%})$ from $\mathbf{1 d}(467 \mathrm{mg}, 2.01 \mathrm{mmol})$

yellow oil

eluent: cyclohexane/toluene $3: 1$ to $1: 1$, then toluene, then toluene/ethyl acetate $20: 1$ to $5: 1$

${ }^{1} \mathrm{H}$ NMR $\left(400 \mathrm{MHz}, \mathbf{C D C l}_{3}\right): \delta=8.05(\mathrm{~d}, J=8.8 \mathrm{~Hz}, 1 \mathrm{H}), 7.61-7.55(\mathrm{~m}$, $2 \mathrm{H}), 7.51-7.43(\mathrm{~m}, 3 \mathrm{H}), 6.81(\mathrm{~d}, J=8.8 \mathrm{~Hz}, 1 \mathrm{H}), 3.32-3.18(\mathrm{~m}, 2 \mathrm{H})$, $2.07-1.96(\mathrm{~m}, 1 \mathrm{H}), 1.91-1.81(\mathrm{~m}, 1 \mathrm{H}), 1.67-1.49(\mathrm{~m}, 2 \mathrm{H}), 1.07-1.00$ $(\mathrm{m}, 1 \mathrm{H})$.

${ }^{13}$ C NMR (100 MHz, $\left.\mathbf{C D C l}_{\mathbf{3}}\right): \delta=167.4,156.0,142.1,135.7,133.2,130.2$, $129.9,128.6,118.2,61.4,34.6,30.2,28.6,26.7$.

MS (EI): $\mathrm{m} / \mathrm{z}(\%)=372,370,368\left(0,3,5,[\mathrm{M}]^{+}\right), 335,333(12,29,[\mathrm{M}-$ $\left.\mathrm{Cl}]^{+}\right), 272\left(100,\left[\mathrm{M}-\mathrm{C}_{2} \mathrm{H}_{2} \mathrm{Cl}_{2}\right]^{+}\right)$.

HRMS (EI): $\mathrm{m} / \mathrm{z}$ [M] ${ }^{+}$calcd for $\mathrm{C}_{16} \mathrm{H}_{14} \mathrm{O}_{2} \mathrm{~N}_{2} \mathrm{~S}^{35} \mathrm{Cl}_{2}: 368.0153$; found: 368.0151 . 
<smiles>CCCCCCCCc1cc(Cl)ncc1[N+](=O)[O-]</smiles>
2-chloro-4-octyl-5-nitropyridine, $\mathbf{4 q}$

Yield:

$197 \mathrm{mg}(0.73 \mathrm{mmol}, \mathbf{3 6} \%)$ from $\mathbf{1 e}(320 \mathrm{mg}, 2.02 \mathrm{mmol})$

yellowish oil

eluent: cyclohexane/toluene $3: 1$ to $1: 1$, then toluene

${ }^{1} \mathbf{H}$ NMR (400 MHz, $\left.\mathbf{C D C l}_{3}\right): \delta=8.87(\mathrm{~s}, 1 \mathrm{H}), 7.30(\mathrm{~s}, 1 \mathrm{H}), 2.92-2.85$ $(\mathrm{m}, 2 \mathrm{H}), 1.66-1.55(\mathrm{~m}, 2 \mathrm{H}), 1.41-1.17(\mathrm{~m}, 10 \mathrm{H}), 0.84(\mathrm{t}, J=7.0 \mathrm{~Hz}$, $3 \mathrm{H})$.

${ }^{13} \mathrm{C}$ NMR (100 MHz, $\left.\mathbf{C D C l}_{3}\right): \delta=155.1,150.0,146.2,144.8,126.1,32.4$, $31.7,29.6,29.4,29.1,29.0,22.6,14.0$.

MS (EI): m/z (\%): 272, $270\left(2,4,[\mathrm{M}]^{+}\right), 255,253\left(15,45,[\mathrm{M}-\mathrm{OH}]^{+}\right), 235$ $\left(47,[\mathrm{M}-\mathrm{Cl}]^{+}\right), 171,169\left(17,49,\left[\mathrm{M}-\mathrm{OC}_{6} \mathrm{H}_{13}\right]^{+}\right), 44\left(100,\left[\mathrm{C}_{3} \mathrm{H}_{8}\right]^{+}\right)$.

HRMS (EI): $\mathrm{m} / \mathrm{z}[\mathrm{M}]^{+}$calcd for $\mathrm{C}_{13} \mathrm{H}_{19} \mathrm{~N}_{2} \mathrm{O}_{2}{ }^{35} \mathrm{Cl}$ : 270.1135; found: 270.1134 . 
<smiles>CCCCCCCc1nc(Oc2c(Cl)cc(Cl)cc2Cl)ccc1[N+](=O)[O-]</smiles>

6-(2,4,6-trichlorophenoxy)-2-octyl-3-nitropyridine, $\mathbf{4 r}$

Yield:

$435 \mathrm{mg}$ (1.01 mmol, 50\%) from $\mathbf{1 f}(642 \mathrm{mg}, 2.01 \mathrm{mmol})$

pale yellowish oil

eluent: cyclohexane/toluene 3:1 to $1: 2$, then toluene

${ }^{1} \mathbf{H}$ NMR $\left(400 \mathrm{MHz}, \mathbf{C D C l}_{3}\right): \delta=8.35(\mathrm{~d}, J=8.9 \mathrm{~Hz}, 1 \mathrm{H}), 7.38(\mathrm{~s}, 2 \mathrm{H})$, $6.99(\mathrm{~d}, J=8.9 \mathrm{~Hz}, 1 \mathrm{H}), 2.92(\mathrm{t}, J=7.3 \mathrm{~Hz}, 2 \mathrm{H}), 1.55-1.46(\mathrm{~m}, 2 \mathrm{H}), 1.30$ $-1.09(\mathrm{~m}, 10 \mathrm{H}), 0.86(\mathrm{t}, J=7.1 \mathrm{~Hz}, 3 \mathrm{H})$.

${ }^{13}$ C NMR (100 MHz, CDCl 3$): \delta=161.7,157.8,144.8,141.8,137.3,131.5$, 130.1, 128.5, 108.2, 35.0, 31.8, 29.4, 29.1, 29.0, 27.4, 22.7, 14.1.

HRMS (ESI): $\mathrm{m} / \mathrm{z}[\mathrm{M}+\mathrm{H}]^{+}$calcd for $\mathrm{C}_{19} \mathrm{H}_{22} \mathrm{~N}_{2} \mathrm{O}_{3}{ }^{35} \mathrm{Cl}_{3}$ : 431.0696; found: 431.0692 .<smiles>CCCCCCCc1cc(Oc2c(Cl)cc(Cl)cc2Cl)ncc1[N+](=O)[O-]</smiles>

2-(2,4,6-trichlorophenoxy)-4-octyl-5-nitropyridine, $\mathbf{4 r}$ '

Yield:

$225 \mathrm{mg}$ (0.52 mmol, 26\%) from $\mathbf{1 f}(642 \mathrm{mg}, 2.01 \mathrm{mmol})$

off-white solid, $\mathbf{m p} 51.5-53.0^{\circ} \mathrm{C}$

eluent: cyclohexane/toluene $3: 1$ to $1: 2$, then toluene

${ }^{1} \mathbf{H}$ NMR (400 MHz, $\left.\mathbf{C D C l}_{3}\right): \delta=8.70(\mathrm{~s}, 1 \mathrm{H}), 7.40(\mathrm{~s}, 2 \mathrm{H}), 7.02(\mathrm{~s}, 1 \mathrm{H})$, $3.01-2.96(\mathrm{~m}, 2 \mathrm{H}), 1.73-1.63(\mathrm{~m}, 2 \mathrm{H}), 1.46-1.22(\mathrm{~m}, 10 \mathrm{H}), 0.87(\mathrm{t}, J$ $=7.0 \mathrm{~Hz}, 3 \mathrm{H})$.

${ }^{13}$ C NMR (100 MHz, $\left.\mathbf{C D C l}_{3}\right): \delta=163.4,152.0,145.7,144.5,142.5,131.9$, 130.0, 128.8, 111.8, 33.1, 31.8, 29.51, 29.48, 29.2, 29.1, 22.6, 14.1.

HRMS (ESI): $\mathrm{m} / \mathrm{z}[\mathrm{M}+\mathrm{H}]^{+}$calcd for $\mathrm{C}_{19} \mathrm{H}_{22} \mathrm{~N}_{2} \mathrm{O}_{3}{ }^{35} \mathrm{Cl}_{3}: 431.0696$; found: 431.0681 . 

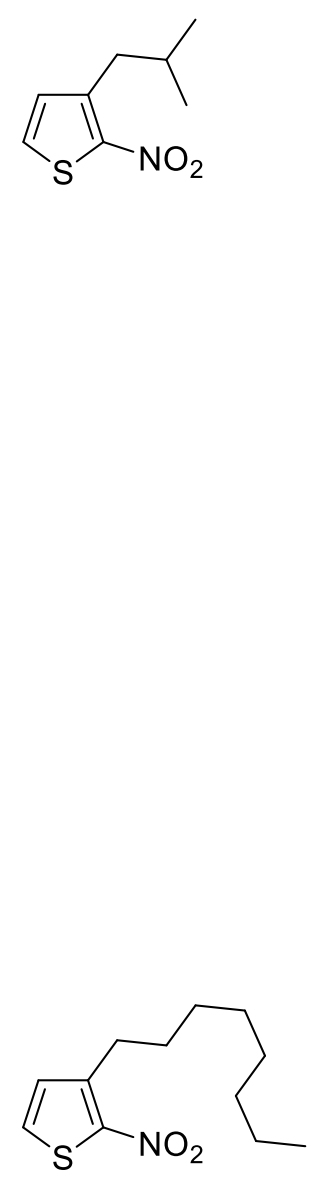

3-isobutyl-2-nitrothiophene, $4 \mathrm{~s}$

Yield:

$204 \mathrm{mg}$ (1.10 mmol, 55\%) from $\mathbf{1 g}(259 \mathrm{mg}, 2.01 \mathrm{mmol})$

orange oil

eluent: cyclohexane/toluene 5:1 to $1: 2$

${ }^{1} \mathbf{H}_{\text {NMR }}\left(\mathbf{4 0 0} \mathbf{M H z}, \mathbf{C D C l}_{3}\right): \delta=7.38(\mathrm{~d}, J=5.4 \mathrm{~Hz}, 1 \mathrm{H}), 6.87(\mathrm{~d}, J=$ $5.4 \mathrm{~Hz}, 1 \mathrm{H}), 2.90(\mathrm{~d}, J=7.2 \mathrm{~Hz}, 2 \mathrm{H}), 2.06-1.88(\mathrm{~m}, 1 \mathrm{H}), 0.91(\mathrm{~d}, J=6.7$ $\mathrm{Hz}, 6 \mathrm{H})$.

${ }^{13} \mathbf{C}$ NMR (100 MHz, $\left.\mathbf{C D C l}_{3}\right): \delta=147.0,145.4,131.0,129.9,38.2,29.0$, 22.4 .

MS (EI): m/z (\%): $186\left(1,[\mathrm{M}+\mathrm{H}]^{+}\right), 185\left(5,[\mathrm{M}]^{+}\right), 168\left(38,[\mathrm{M}-\mathrm{OH}]^{+}\right), 126$ $\left(100,\left[\mathrm{M}-\mathrm{OC}_{3} \mathrm{H}_{7}\right]^{+}\right), 44\left(67,\left[\mathrm{C}_{3} \mathrm{H}_{8}\right]^{+}\right)$.

HRMS (EI): m/z [M] $]^{+}$calcd for $\mathrm{C}_{8} \mathrm{H}_{11} \mathrm{NO}_{2} \mathrm{~S}: 185.0511$; found: 185.0507 .

3-octyl-2-nitrothiophene, $\mathbf{4 t}$

Yield:

$279 \mathrm{mg}$ (1.16 mmol, 58\%) from $\mathbf{1 g}(259 \mathrm{mg}, 2.01 \mathrm{mmol})$

yellow oil

eluent: cyclohexane/toluene 20:1 to 4:1

${ }^{1}$ H NMR (400 MHz, CDCl $): \delta=7.37(\mathrm{~d}, J=5.5 \mathrm{~Hz}, 1 \mathrm{H}), 6.90(\mathrm{~d}, J=$ $5.5 \mathrm{~Hz}, 1 \mathrm{H}), 3.02-2.97(\mathrm{~m}, 2 \mathrm{H}), 1.66-1.57(\mathrm{~m}, 2 \mathrm{H}), 1.39-1.17(\mathrm{~m}$, $10 \mathrm{H}), 0.84(\mathrm{t}, J=6.9 \mathrm{~Hz}, 3 \mathrm{H})$.

${ }^{13} \mathbf{C}$ NMR (100 MHz, $\left.\mathbf{C D C l}_{3}\right): \delta=146.6,146.5,130.2,130.1,31.7,29.6$, $29.4,29.21,29.19,29.1,22.5,14.0$.

MS (EI): m/z (\%): $241\left(1,[\mathrm{M}]^{+}\right), 224\left(45,[\mathrm{M}-\mathrm{OH}]^{+}\right), 97\left(100,\left[\mathrm{C}_{7} \mathrm{H}_{13}\right]^{+}\right)$, $44\left(55,\left[\mathrm{C}_{3} \mathrm{H}_{8}\right]^{+}\right)$.

HRMS (EI): $\mathrm{m} / \mathrm{z}$ [M] $]^{+}$calcd for $\mathrm{C}_{12} \mathrm{H}_{19} \mathrm{NO}_{2} \mathrm{~S}$ : 241.1137; found: 241.1134 . 
<smiles>Cc1c([N+](=O)[O-])cnc2ccccc12</smiles><smiles>CCCCCCCCc1cc(Cl)ccc1[N+](=O)[O-]</smiles>

4-methyl-3-nitroquinoline, $\mathbf{4 u}$

Yield:

$147 \mathrm{mg}(0.78 \mathrm{mmol}, \mathbf{7 9 \%})$ from $\mathbf{1 h}(172 \mathrm{mg}, 0.99 \mathrm{mmol})$

white solid, mp $115.5-116.5^{\circ} \mathrm{C}$

eluent: toluene/ethyl acetate 50:1 to 5:1

${ }^{1}$ H NMR (400 MHz, CDCl $): \delta=9.16(\mathrm{~s}, 1 \mathrm{H}), 8.15-8.10(\mathrm{~m}, 2 \mathrm{H}), 7.82$ $(\mathrm{ddd}, J=8.4,6.9,1.4 \mathrm{~Hz}, 1 \mathrm{H}), 7.67$ (ddd, $J=8.4,6.9,1.3 \mathrm{~Hz}, 1 \mathrm{H}), 2.86$ $(\mathrm{s}, 3 \mathrm{H})$.

${ }^{13}$ C NMR (100 MHz, CDCl $): ~ \delta=148.3,144.4,143.3,140.1,131.9,130.4$, $128.4,126.8,125.4,14.2$.

MS (EI): m/z (\%): $189\left(18,[\mathrm{M}+\mathrm{H}]^{+}\right), 188\left(78,[\mathrm{M}]^{+}\right), 171\left(70,[\mathrm{M}-\mathrm{OH}]^{+}\right)$, $142\left(41,\left[\mathrm{M}-\mathrm{NO}_{2}\right]^{+}\right), 115\left(100,\left[\mathrm{M}-\mathrm{HCN}-\mathrm{NO}_{2}\right]^{+}\right)$.

HRMS (EI): $\mathrm{m} / \mathrm{z}[\mathrm{M}]^{+}$calcd for $\mathrm{C}_{10} \mathrm{H}_{8} \mathrm{~N}_{2} \mathrm{O}_{2}$ : 188.0586; found: 188.0591 .

4-chloro-2-octylnitrobenzene, $\mathbf{4 v}$

Yield:

$81 \mathrm{mg}(0.30 \mathrm{mmol}, \mathbf{1 5 \%})$ from $1 \mathbf{i}(317 \mathrm{mg}, 2.01 \mathrm{mmol})$

orange oil

eluent: cyclohexane/toluene 20:1 to 5:1

${ }^{1} \mathbf{H}$ NMR $\left(400 \mathrm{MHz}, \mathbf{C D C l}_{3}\right): \delta=7.83(\mathrm{~d}, J=8.6 \mathrm{~Hz}, 1 \mathrm{H}), 7.32-7.25(\mathrm{~m}$, $2 \mathrm{H}), 2.86-2.81(\mathrm{~m}, 2 \mathrm{H}), 1.65-1.55(\mathrm{~m}, 2 \mathrm{H}), 1.40-1.19(\mathrm{~m}, 10 \mathrm{H}), 0.88$ $-0.83(\mathrm{t}, J=6.9 \mathrm{~Hz}, 3 \mathrm{H})$.

${ }^{13}$ C NMR (100 MHz, CDCl $\left._{3}\right): \delta=147.5,139.9,139.0,131.6,126.9,126.2$, $33.0,31.8,30.5,29.5,29.2,29.1,22.6,14.1$.

MS (EI): m/z (\%): 271, 269 (3, 9, [M] $\left.]^{+}\right), 254,252\left(48,100,[\mathrm{M}-\mathrm{OH}]^{+}\right), 234$ $\left(20,[\mathrm{M}-\mathrm{Cl}]^{+}\right), 156,154\left(35,74,\left[\mathrm{M}-\mathrm{OC}_{7} \mathrm{H}_{15}\right]^{+}\right)$.

HRMS (EI): $\mathrm{m} / \mathrm{z}[\mathrm{M}]^{+}$calcd for $\mathrm{C}_{14} \mathrm{H}_{20} \mathrm{NO}_{2}{ }^{35} \mathrm{Cl}$ : 269.1183; found: 269.1195 . 


\subsection{Characterization of protonated $\sigma^{\mathrm{H}}$-adducts}<smiles>CC(C)([C@H]1C=CNC=C1[N+](=O)[O-])[Sb](=O)(Oc1ccccc1)Oc1ccccc1</smiles><smiles>C[C@H](Oc1ccccc1)C1C([N+](=O)[O-])=CNc2ccccc21</smiles>

$5 \mathbf{a}$

Yield:

$266 \mathrm{mg}(0.86 \mathrm{mmol}, \mathbf{4 3 \%})$ from $\mathbf{1 a}(251 \mathrm{mg}, 2.02 \mathrm{mmol})$

orange solid, $\mathbf{m p} 157.0^{\circ} \mathrm{C}$ (dec.)

eluent: cyclohexane/ethyl acetate 5:1 to 1:2, then ethyl acetate

${ }^{1}$ H NMR (400 MHz, DMSO-d $\mathbf{6}_{\mathbf{6}}$ ): $\delta=9.68$ (br s, $1 \mathrm{H}$ ), 8.24 (ddd, $J=6.7$, $1.6,0.7 \mathrm{~Hz}, 1 \mathrm{H}), 7.89-7.84(\mathrm{~m}, 2 \mathrm{H}), 7.82-7.76(\mathrm{~m}, 1 \mathrm{H}), 7.71-7.65(\mathrm{~m}$, 2H), 6.56 (ddd, $J=7.6,4.3,0.7 \mathrm{~Hz}, 1 \mathrm{H}), 5.34(\mathrm{ddd}, J=7.6,6.3,1.7 \mathrm{~Hz}$, $1 \mathrm{H}), 4.64(\mathrm{dd}, J=6.3,1.6 \mathrm{~Hz}, 1 \mathrm{H}), 1.12$ (s, 3H), 0.90 (s, 3H).

${ }^{13}$ C NMR (100 MHz, DMSO- $\left.d_{6}\right): \delta=140.4,136.1,134.6,130.5,129.7$, $126.8,117.6,106.6,69.3,36.6,20.0,17.4$.

HRMS (ESI): $\mathrm{m} / \mathrm{z}$ [M+Na] ${ }^{+}$calcd for $\mathrm{C}_{14} \mathrm{H}_{16} \mathrm{~N}_{2} \mathrm{O}_{4} \mathrm{SNa}$ : 331.0728; found: 331.0725 .

$\mathbf{5 b}$

Yield:

$117 \mathrm{mg}(0.34 \mathrm{mmol}, \mathbf{6 8 \%})$ from $\mathbf{1 h}(87 \mathrm{mg}, 0.50 \mathrm{mmol})$

yellow solid, $\mathbf{m p} 153.0^{\circ} \mathrm{C}$ (dec.)

eluent: toluene/ethyl acetate $10: 1$ to $1: 2$, then ethyl acetate

${ }^{1}$ H NMR (400 MHz, DMSO- $\left.\boldsymbol{d}_{6}\right): \delta=10.65(\mathrm{~d}, J=6.8 \mathrm{~Hz}, 1 \mathrm{H}), 8.37(\mathrm{dd}$, $J=6.8,0.6 \mathrm{~Hz}, 1 \mathrm{H}), 7.88-7.83(\mathrm{~m}, 2 \mathrm{H}), 7.78-7.72(\mathrm{~m}, 1 \mathrm{H}), 7.70-7.64$ $(\mathrm{m}, 2 \mathrm{H}), 7.55(\mathrm{~d}, J=7.0 \mathrm{~Hz}, 1 \mathrm{H}), 7.34-7.28(\mathrm{~m}, 1 \mathrm{H}), 7.21-7.09(\mathrm{~m}, 2 \mathrm{H})$, $5.34(\mathrm{~s}, 1 \mathrm{H}), 3.40(\mathrm{qd}, J=7.1,1.5 \mathrm{~Hz}, 1 \mathrm{H}), 0.83(\mathrm{~d}, J=7.1 \mathrm{~Hz}, 3 \mathrm{H})$.

${ }^{13}$ C NMR (100 MHz, DMSO- $\left.\boldsymbol{d}_{\boldsymbol{6}}\right): \delta=140.2,139.2,136.8,134.4,131.8$, $130.0,129.1,128.5,125.2,119.9,119.3,117.1,61.7,36.1,9.2$.

MS (EI): m/z (\%): $344\left(5,[\mathrm{M}]^{+}\right), 327\left(2,[\mathrm{M}-\mathrm{OH}]^{+}\right), 202(14,[\mathrm{M}-$ $\left.\left.\mathrm{PhSO}_{2} \mathrm{H}\right]^{+}\right), 175\left(100,\left[\mathrm{M}-\mathrm{PhSO}_{2} \mathrm{C}_{2} \mathrm{H}_{4}\right]^{+}\right), 129\left(56,\left[\mathrm{M}-\mathrm{PhSO}_{2} \mathrm{C}_{2} \mathrm{H}_{4}-\mathrm{NO}_{2}\right]^{+}\right)$.

HRMS (EI): $\mathrm{m} / \mathrm{z}$ [M] ${ }^{+}$calcd for $\mathrm{C}_{17} \mathrm{H}_{16} \mathrm{~N}_{2} \mathrm{O}_{4} \mathrm{~S}: 344.0831$; found: 344.0840 . 
<smiles>CC(C)(Oc1ccccc1)C1C([N+](=O)[O-])=CNc2ccccc21</smiles>
$5 c$

Yield:

$19 \mathrm{mg}(0.05 \mathrm{mmol}, \mathbf{1 1 \%})$ from $\mathbf{1 h}(87 \mathrm{mg}, 0.50 \mathrm{mmol})$

yellow oil

eluent: toluene/ethyl acetate $20: 1$ to $1: 1$, then ethyl acetate

${ }^{1} \mathbf{H}_{\text {NMR }}\left(\mathbf{4 0 0} \mathbf{~ M H z}, \mathbf{C D C l}_{3}\right): \delta=8.46(\mathrm{~s}, 2 \mathrm{H}), 7.85-7.79(\mathrm{~m}, 2 \mathrm{H}), 7.64$ $-7.57(\mathrm{~m}, 2 \mathrm{H}), 7.54-7.46(\mathrm{~m}, 2 \mathrm{H}), 7.25-7.20(\mathrm{~m}, 1 \mathrm{H}), 7.15(\mathrm{td}, \mathrm{J}=7.5$, $1.2 \mathrm{~Hz}, 1 \mathrm{H}), 7.06(\mathrm{~d}, \mathrm{~J}=7.9 \mathrm{~Hz}, 1 \mathrm{H}), 5.55(\mathrm{~s}, 1 \mathrm{H}), 1.06(\mathrm{~s}, 3 \mathrm{H}), 0.96(\mathrm{~s}$, $3 \mathrm{H})$.

${ }^{13}$ C NMR (100 MHz, $\left.\mathbf{C D C l}_{3}\right): \delta=139.3,136.9,136.4,133.8,132.2,130.4$, $128.8,128.3,124.8,120.2,119.2,116.3,68.6,40.0,20.4,20.1$.

HRMS (ESI): $\mathrm{m} / \mathrm{z}$ [M+Na] ${ }^{+}$calcd for $\mathrm{C}_{18} \mathrm{H}_{18} \mathrm{~N}_{2} \mathrm{O}_{4} \mathrm{SNa}$ : 381.0885; found: 381.0874 .

For other components of reaction mixtures, from which adducts 5a-c were isolated, see table at S-24 (entries 26, 27 and 29). 


\subsection{Characterization of side products}

Alkylation of less electrophilic nitroarene $1 \mathbf{i}$ resulted in the formation of the alkylated product $(\mathbf{4 v}, 15 \%)$ along with two side products - dimer $(\mathbf{6 a}, 39 \%)$ and ONSH product $(\mathbf{1 0}, 10 \%)$. Moreover substrate 1i was recovered in $28 \%$.<smiles>CCCCCCCCS(=O)(=O)OCC(C)(C)C</smiles><smiles>CCCCCCC[C@H](c1cc(Cl)ccc1[N+](=O)[O-])[C@H](CCCCCC)c1cc(Cl)ccc1[N+](=O)[O-]</smiles>

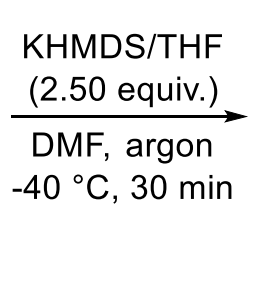<smiles>CCCCCCCc1cc(Cl)ccc1[N+](=O)[O-]</smiles>

$4 \mathbf{v}, 15 \%$<smiles>CCCCCCCCCCCCCCC[C@H](c1cc(Cl)ccc1[N+](=O)[O-])[C@H](CCCCCCC)c1cc(Cl)ccc1[N+](=O)[O-]</smiles><smiles>CCCCCCCC(CC(C)(C)C)c1cc(Cl)ccc1[N+](=O)[O-]</smiles>

$10,10 \%$

$+28 \%$ of $1 i$ recovered

6a

\section{Yield:}

$208 \mathrm{mg}$ (0.39 mmol, 39\%) from $1 \mathbf{i}(317 \mathrm{mg}, 2.01 \mathrm{mmol})$

orange oil

eluent: cyclohexane/toluene $20: 1$ to $1: 1$, then toluene, then toluene/ethyl acetate $50: 1$ to $20: 1$

${ }^{1} \mathbf{H}$ NMR (400 MHz, $\left.\mathbf{C D C l}_{3}\right): \delta=7.39(\mathrm{~d}, J=8.7 \mathrm{~Hz}, 2 \mathrm{H}), 7.27(\mathrm{~d}, J=$ $2.2 \mathrm{~Hz}, 2 \mathrm{H}), 7.10(\mathrm{dd}, J=8.7,2.2 \mathrm{~Hz}, 2 \mathrm{H}), 3.57(\mathrm{~s}, 2 \mathrm{H}), 2.12-2.00(\mathrm{~m}$, $2 \mathrm{H}), 1.64-1.49(\mathrm{~m}, 2 \mathrm{H}), 1.30-1.12(\mathrm{~m}, 18 \mathrm{H}), 1.02-0.90(\mathrm{~m}, 2 \mathrm{H}), 0.83$ (t, $J=7.0 \mathrm{~Hz}, 6 \mathrm{H})$.

${ }^{13} \mathbf{C}$ NMR (100 MHz, $\left.\mathbf{C D C l}_{3}\right): \delta=149.0,140.0,139.1,128.6,127.2,125.1$, $45.1,34.7,31.7,29.6,29.0,27.1,22.5,14.0$.

HRMS (ESI): $\mathrm{m} / \mathrm{z}[\mathrm{M}+\mathrm{Na}]^{+}$calcd for $\mathrm{C}_{28} \mathrm{H}_{38} \mathrm{~N}_{2} \mathrm{O}_{4}{ }^{35} \mathrm{Cl}_{2} \mathrm{Na}$ : 559.2106; found: 559.2111 . 
<smiles>CCCCCCCC(OS(=O)(=O)CC(C)(C)C)c1cc(Cl)ccc1[N+](=O)[O-]</smiles>

Yield:

$82 \mathrm{mg}(0.20 \mathrm{mmol}, \mathbf{1 0 \%})$ from $1 \mathbf{i}(317 \mathrm{mg}, 2.01 \mathrm{mmol})$

orange oil

eluent: cyclohexane/toluene $20: 1$ to $1: 1$, then toluene, then toluene/ethyl acetate 50:1 to $20: 1$

${ }^{1} \mathrm{H}$ NMR (400 MHz, $\left.\mathbf{C D C l}_{3}\right): \delta=7.89(\mathrm{~d}, J=8.8 \mathrm{~Hz}, 1 \mathrm{H}), 7.77(\mathrm{~d}, J=$ $2.2 \mathrm{~Hz}, 1 \mathrm{H}), 7.48(\mathrm{dd}, J=8.8,2.2 \mathrm{~Hz}, 1 \mathrm{H}), 5.35(\mathrm{dd}, J=10.6,4.4 \mathrm{~Hz}, 1 \mathrm{H})$, $3.80(\mathrm{~d}, J=9.1 \mathrm{~Hz}, 1 \mathrm{H}), 3.73(\mathrm{~d}, J=9.1 \mathrm{~Hz}, 1 \mathrm{H}), 2.45-2.34(\mathrm{~m}, 1 \mathrm{H})$, $2.19-2.06(\mathrm{~m}, 1 \mathrm{H}), 1.40-1.13(\mathrm{~m}, 10 \mathrm{H}), 0.86-0.81(\mathrm{~m}, 12 \mathrm{H})$.

${ }^{13}$ C NMR (100 MHz, $\left.\mathbf{C D C l}_{3}\right): \delta=148.8,139.8,130.0,129.79,129.75$, $126.5,79.4,58.7,31.7,31.6,30.3,28.9,28.8,26.3,25.9,22.5,14.0$.

HRMS (ESI): $\mathrm{m} / \mathrm{z}[\mathrm{M}+\mathrm{Na}]^{+}$calcd for $\mathrm{C}_{19} \mathrm{H}_{30} \mathrm{NO}_{5} \mathrm{~S}^{35} \mathrm{ClNa}$ : 442.1431; found: 442.1429 .

From reaction of $\mathbf{1 i}$ with $\mathbf{3 b}$ under standard conditions we isolated dimer $\mathbf{6 b}$ :<smiles>CC(c1cc(Cl)ccc1[N+](=O)[O-])C(C)c1cc(Cl)ccc1[N+](=O)[O-]</smiles>

6b

Yield:

$85 \mathrm{mg}$ (0.23 mmol, 23\%) from 1i (317 mg, $2.01 \mathrm{mmol})$

white crystals, $\mathbf{m p} 153.0^{\circ} \mathrm{C}$ (dec.)

eluent: cyclohexane/toluene 20:1 to $1: 1$, then toluene, then toluene/ethyl acetate $20: 1$ to $1: 1$

${ }^{1}$ H NMR (400 MHz, $\left.\mathbf{C D C l}_{3}\right): \delta=7.43(\mathrm{~d}, J=8.7 \mathrm{~Hz}, 2 \mathrm{H}), 7.25(\mathrm{~d}, J=$ $2.2 \mathrm{~Hz}, 2 \mathrm{H}), 7.12(\mathrm{dd}, J=8.7,2.2 \mathrm{~Hz}, 2 \mathrm{H}), 3.63-3.53(\mathrm{~m}, 2 \mathrm{H}), 1.45-$ $1.42(\mathrm{~m}, 6 \mathrm{H})$.

${ }^{13} \mathbf{C}$ NMR (100 MHz, $\left.\mathbf{C D C l}_{3}\right): \delta=147.9,141.3,139.2,128.2,127.3,125.2$, 40.0, 20.4.

HRMS (ESI): $\mathrm{m} / \mathrm{z}[\mathrm{M}+\mathrm{Na}]^{+}$calcd for $\mathrm{C}_{16} \mathrm{H}_{14} \mathrm{~N}_{2} \mathrm{O}_{4}{ }^{35} \mathrm{Cl}_{2} \mathrm{Na}$ : 391.0228; found: 391.0229 . 
Formation of products $6 \mathbf{a}$ and $\mathbf{1 0}$ requires formal oxidation step, therefore reaction of $1 \mathbf{i}$ with $\mathbf{3 e}$ was repeated with excess of $\mathbf{1 i}$, which can serve as an oxidant. Surprisingly, in this experiment yields of alkylated nitroarene $(\mathbf{4 v}, 15 \%)$ and its dimer $(\mathbf{6 a}, 39 \%)$ remained unchanged, while yield of ONSH product (10) significantly increased (44\% versus $10 \%)$.<smiles>CCCCCCCCCCCC[C@@H](c1cc(Cl)ccc1C(CCCCCCC)c1cc(Cl)ccc1N(C(=O)OCC(C)(C)C)C(C)(C)C)[C@@H](CCCCCCC)c1cc(Cl)ccc1[N+](=O)[O-]</smiles>

a - yield calculated on 1.00 equiv. of $1 \mathbf{i}$

To reveal mechanism of the dimerization process $(4 \mathbf{v} \rightarrow \mathbf{6 a})$ reaction of $1 \mathbf{i}$ with $3 \mathbf{e}$ was repeated with 1.50 equiv. of TEMPO, as a radical scavenger. Again, surprisingly yield of dimer $(\mathbf{6 a}, 39 \%)$ remained unchanged, while yield of the alkylated product (4v) slightly decreased (10\% versus $15 \%)$, and yield of ONSH product (10) slightly increased (16\% versus $10 \%)$.

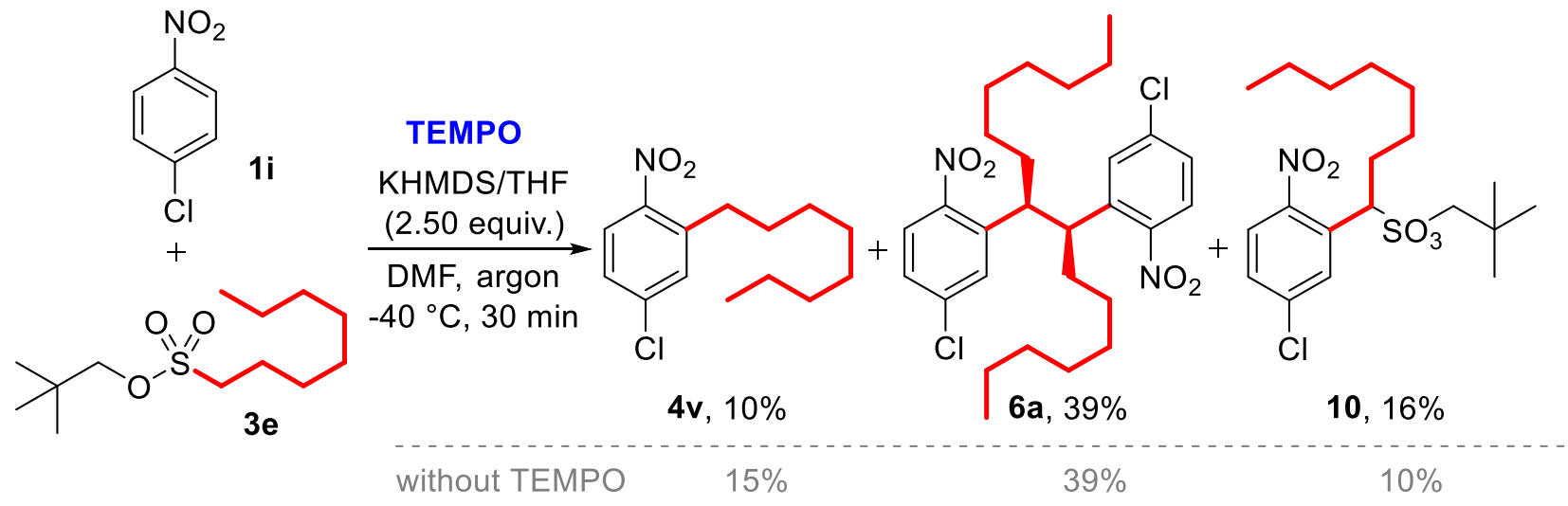




\section{Configuration of dimer 6a}

${ }^{1} \mathrm{H}$ NMR spectra measured for $\mathbf{6 a}$ and $\mathbf{6 b}$ (structure of $\mathbf{6 b}$ confirmed by X-ray) are very similar, confirming that the same diastereoisomer was obtained, as a main product:
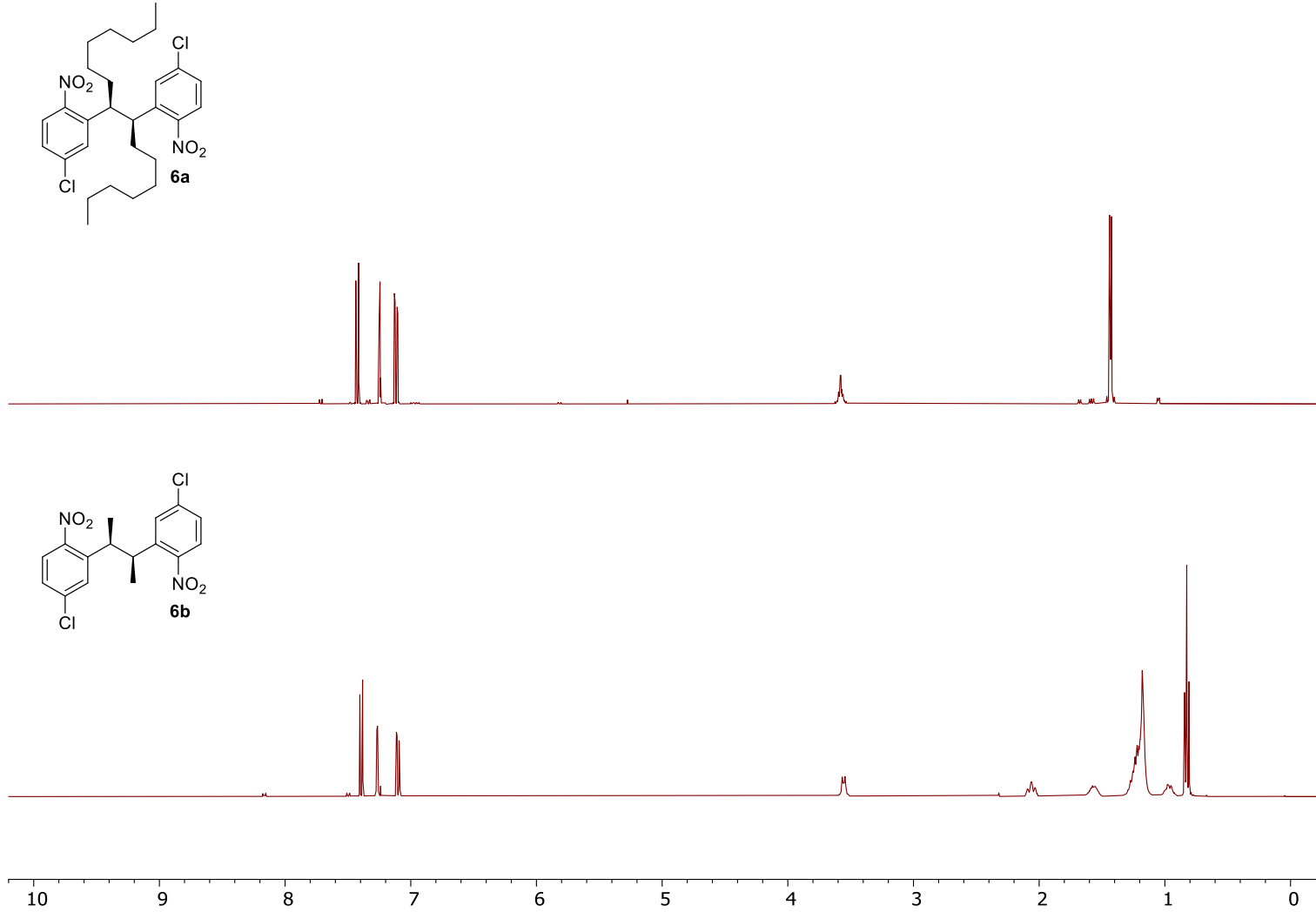

Especially, aromatic signals in $7.50-7.00 \mathrm{ppm}$ range vary less than $0.03 \mathrm{ppm}$ :
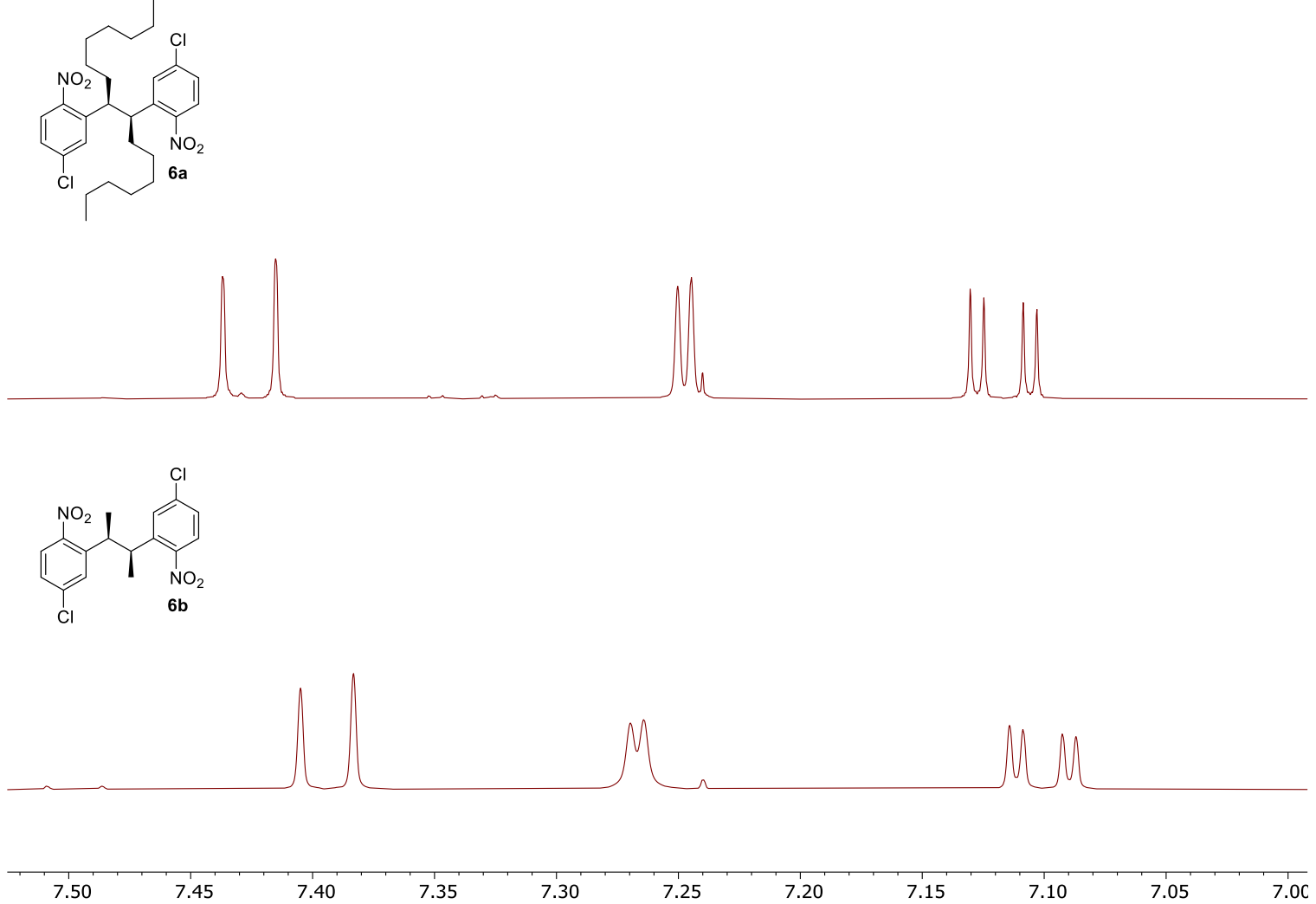


\section{Second diastereoizomer of dimer}

After column chromatography we observed small amount ( $<5 \%$ yield) of minor dimeric product (isomer) inseparable from $\mathbf{6 b}$. Stacked ${ }^{1} \mathrm{H}$ NMR spectra for pure $(\mathbf{6 b})$ and its mixture with the minor dimeric product are shown below (the mixture represents one fraction after chromatography, in which the second isomer was observed):
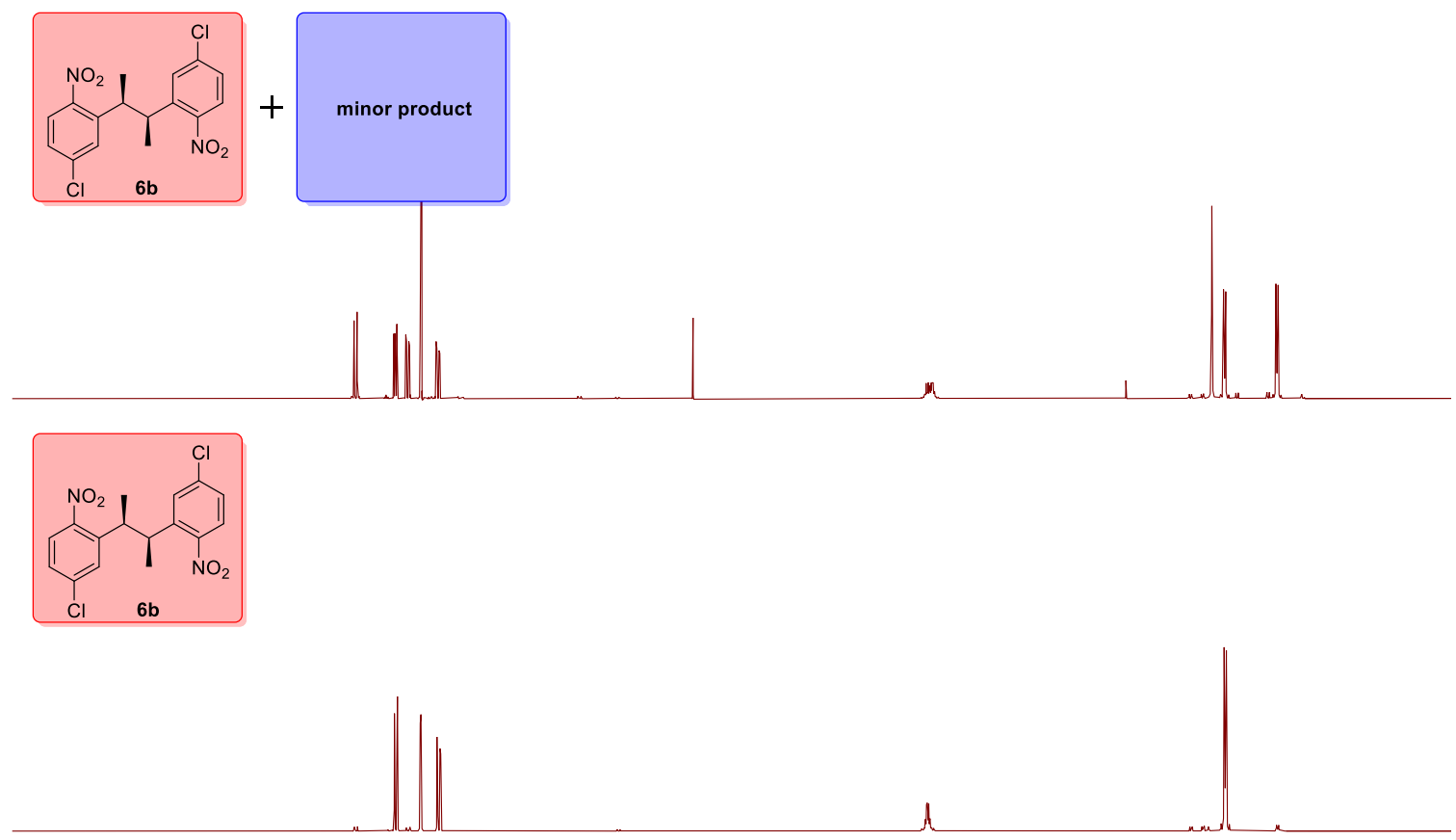

10


In $7.80-7.00$ ppm range set of peaks (7.43, d; 7.25, d; 7.12, dd) arising from (6b) and set of peaks of minor dimeric product shifted ca. 0.25 ppm downfield (7.71, d; 7.44, d; 7.34; dd) were observed:
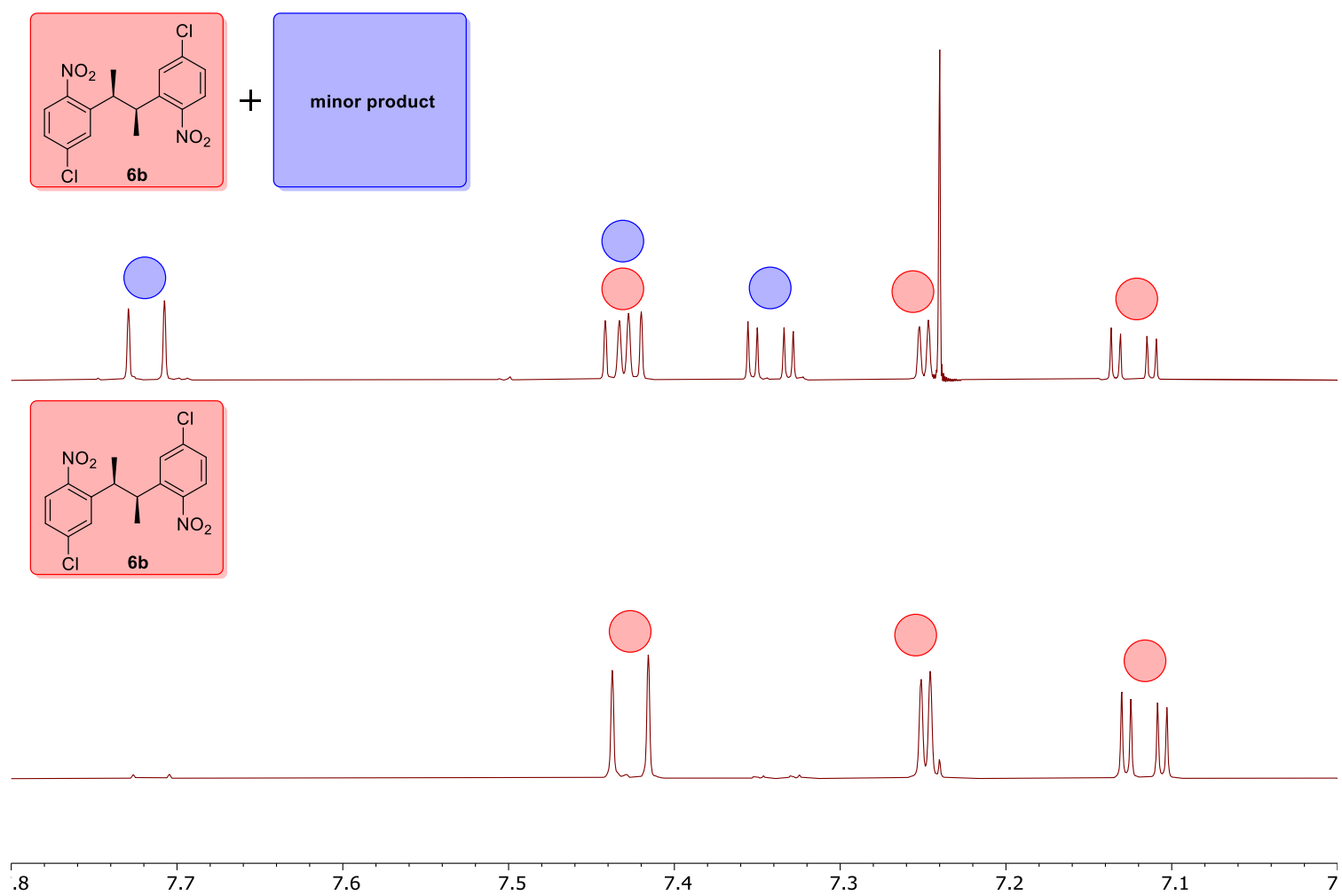

Multiplet around $3.55 \mathrm{ppm}$ arising from minor dimeric product is similar to peak from benzyl protons of $6 \mathbf{b}$ :
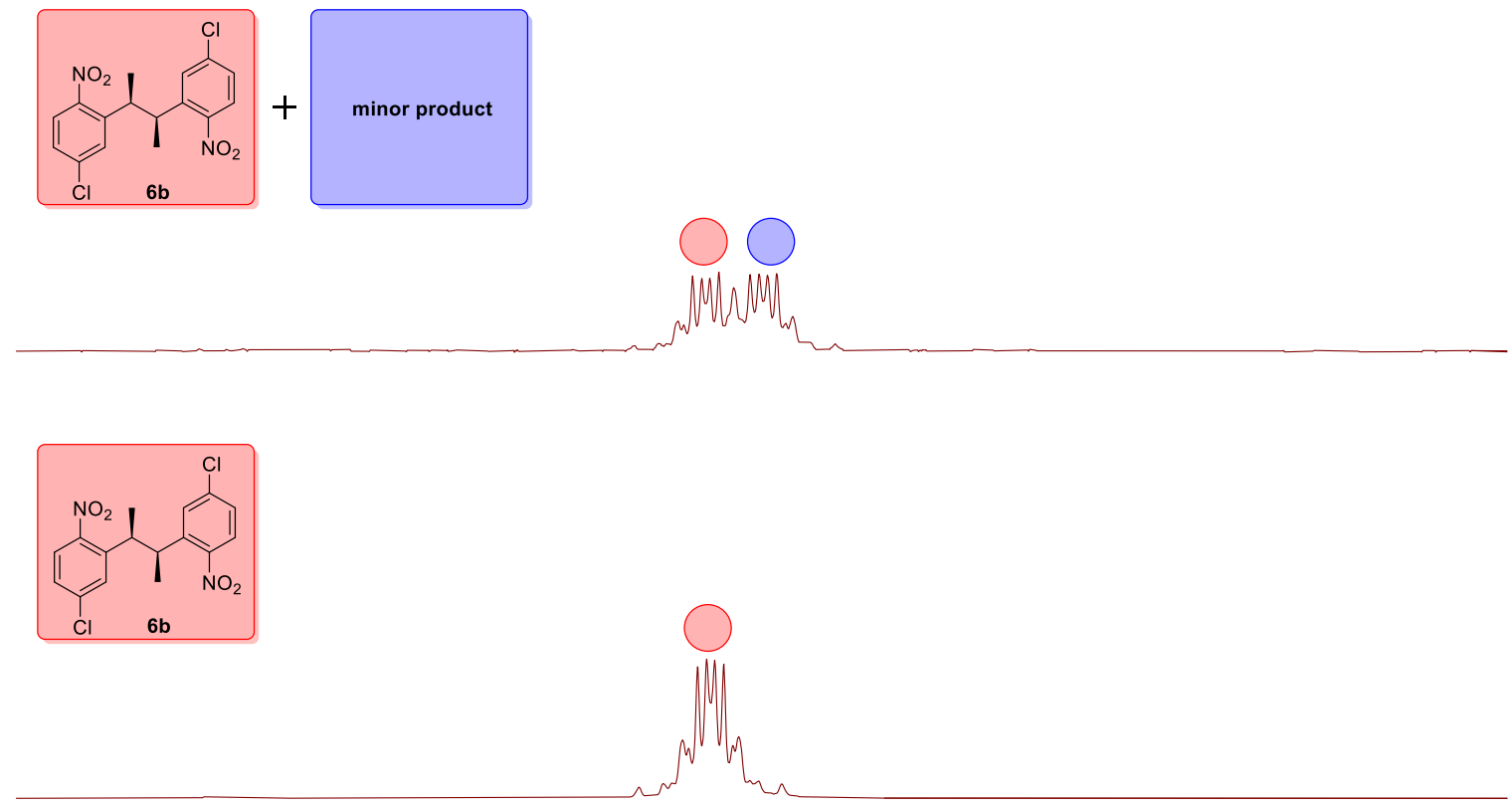

\begin{tabular}{lllllllll}
\hline & 3.0 & 3.9 & 3.7 & 3.6 & 3.5 & 3.4 & 3.3 & 3.2
\end{tabular}


Signal from methyl groups with $\delta=1.43 \mathrm{ppm}$ from $(\mathbf{6 b})$ and similar peak with $\delta=1.06 \mathrm{ppm}$ from minor product were also observed:
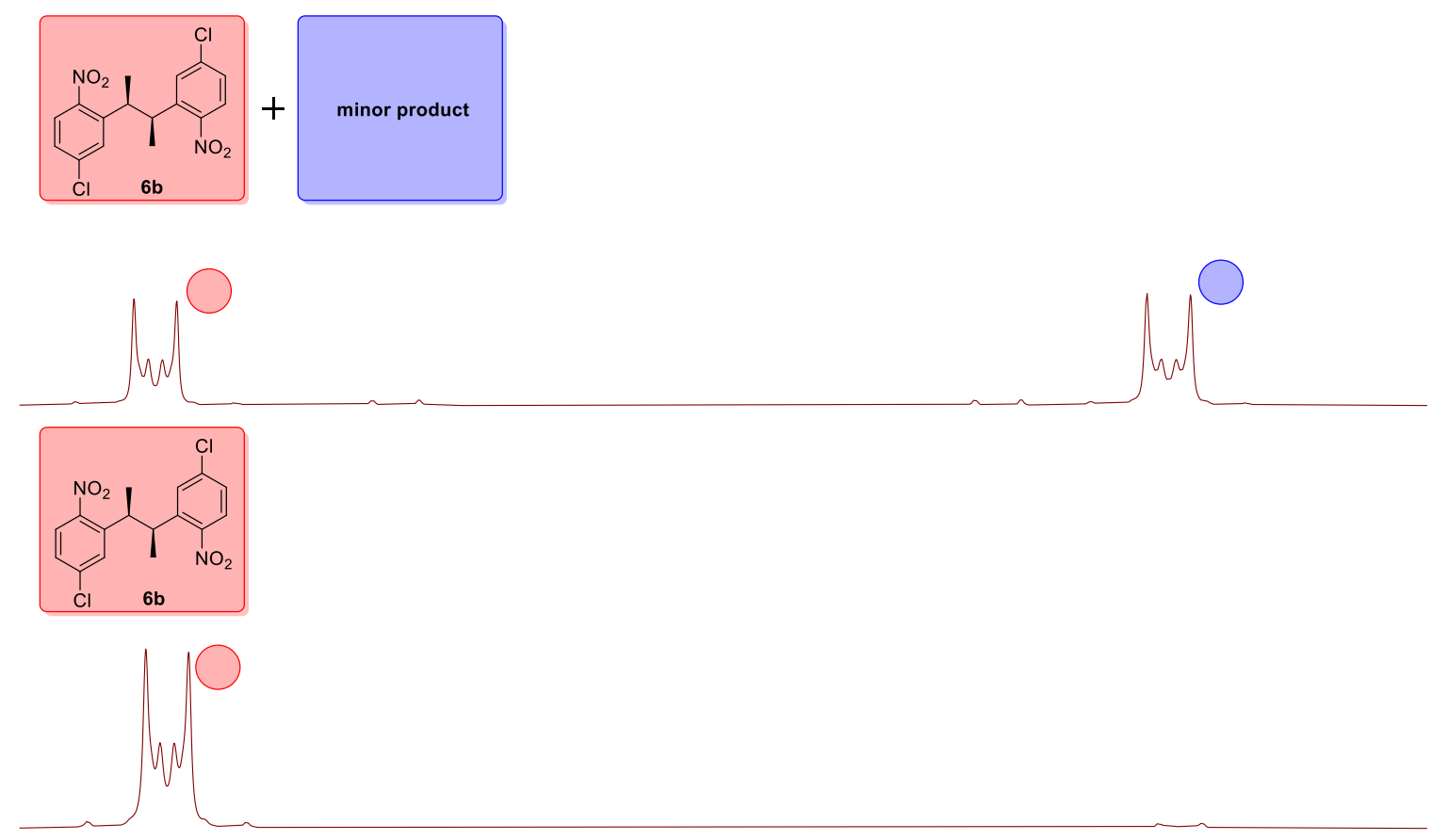

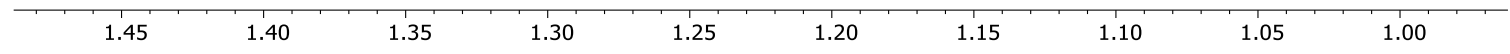

Based on the spectroscopic data we assume that the dimerization process gives two diastereoisomers of product, and one of them (characterized with X-ray) predominates in the mixture. The same conclusion applies to dimerization of octylated chloronitrobenzene $\mathbf{4 v}$. 
In reaction of 1a with 3a under standard reaction conditions dimer $\mathbf{6 c}$ was isolated, as a byproduct. For studies of dimerization of isolated sample of $\mathbf{4 a}$, see next chapter.<smiles>O=[N+]([O-])c1cnccc1CCc1ccncc1[N+](=O)[O-]</smiles>

6c

Yield:

$67 \mathrm{mg}(0.24 \mathrm{mmol}, \mathbf{2 4 \%})$ from $\mathbf{1 a}(251 \mathrm{mg}, 2.02 \mathrm{mmol})$ and $\mathbf{3 a}$ dark yellow solid, $\mathbf{m p} 164.0^{\circ} \mathrm{C}$ (dec.)

eluent: cyclohexane/ethyl acetate $5: 1$ to $1: 2$, then ethyl acetate, then dichloromethane/methanol 50:1 to 10:1

${ }^{1} \mathbf{H}$ NMR (400 MHz, $\left.\mathbf{C D C l}_{3}\right): \delta=9.19(\mathrm{~s}, 2 \mathrm{H}), 8.73(\mathrm{~d}, J=5.0 \mathrm{~Hz}, 2 \mathrm{H})$, $7.37(\mathrm{~d}, J=5.0 \mathrm{~Hz}, 2 \mathrm{H}), 3.29(\mathrm{~s}, 4 \mathrm{H})$.

${ }^{13}$ C NMR (100 MHz, $\left.\mathbf{C D C l}_{3}\right): \delta=153.7,146.3,145.3,144.1,126.2,32.9$.

HRMS (ESI): $\mathrm{m} / \mathrm{z}[\mathrm{M}+\mathrm{H}]^{+}$calcd for $\mathrm{C}_{12} \mathrm{H}_{11} \mathrm{~N}_{4} \mathrm{O}_{4}$ : 275.0780; found: 275.0766 .

In reactions of $\mathbf{1 a}$ with $\mathbf{2 e}$ and with $\mathbf{3 e}$ under standard reaction conditions dimer $\mathbf{6 d}$ was isolated, as a byproduct. For studies of dimerization of isolated sample of $\mathbf{4 e}$, see next chapter.<smiles>CCCCCCCC(c1ccncc1[N+](=O)[O-])C(CCCCCC)c1ccncc1[N+](=O)[O-]</smiles>

6d

Yield:

$21 \mathrm{mg}(0.04 \mathrm{mmol}, \mathbf{4 \%})$ from $\mathbf{1 a}(248 \mathrm{mg}, 2.00 \mathrm{mmol})$ and $\mathbf{3 e}$ $7 \mathrm{mg}(0.02 \mathrm{mmol}, \mathbf{2 \%})$ from $\mathbf{1 a}(251 \mathrm{mg}, 2.02 \mathrm{mmol})$ and $\mathbf{2 e}$

orange oil

eluent: toluene/ethyl acetate $100: 1$ to $3: 1$, then ethyl acetate

${ }^{1} \mathrm{H}$ NMR (400 MHz, $\left.\mathbf{C D C l}_{3}\right): \delta=8.70(\mathrm{~s}, 2 \mathrm{H}), 8.55(\mathrm{~d}, J=5.3 \mathrm{~Hz}, 2 \mathrm{H})$, $7.29(\mathrm{~d}, J=5.3 \mathrm{~Hz}, 2 \mathrm{H}), 3.75-3.64(\mathrm{~m}, 2 \mathrm{H}), 2.15-2.04(\mathrm{~m}, 2 \mathrm{H}), 1.66-$ $1.53(\mathrm{~m}, 2 \mathrm{H}), 1.32-1.10(\mathrm{~m}, 18 \mathrm{H}), 0.96-0.86(\mathrm{~m}, 2 \mathrm{H}), 0.82(\mathrm{t}, J=7.0$ $\mathrm{Hz}, 6 \mathrm{H})$.

${ }^{13}$ C NMR (100 MHz, $\left.\mathbf{C D C l}_{3}\right): \delta=153.0,147.2,146.8,145.0,122.5,44.1$, $34.7,31.7,29.6,28.9,26.9,22.5,14.0$.

HRMS (ESI): $\mathrm{m} / \mathrm{z}$ [M+Na] $]^{+}$calcd for $\mathrm{C}_{26} \mathrm{H}_{38} \mathrm{~N}_{4} \mathrm{O}_{4} \mathrm{Na}$ : 493.2791; found: 493.2793. 


\section{Further studies}

\subsection{Stability of the alkylated products anions}

In order to determine stability of the alkylated nitroarenes anions we tested 4-chloro-2-octylnitrobenzene (4v), 3-nitro-4-octylpyridine (4e), 4-methyl-3-nitropyridine (4a), and 4-isobutyl-3-nitropyridine (4c) under standard reaction conditions (General Procedure; DMF, $-40{ }^{\circ} \mathrm{C}, 30 \mathrm{~min}$ ). Yields of dimers and recovered alkylated nitroarenes are given below.<smiles>[R]Cc1ccncc1[N+](=O)[O-]</smiles>

alkylated nitroarenes

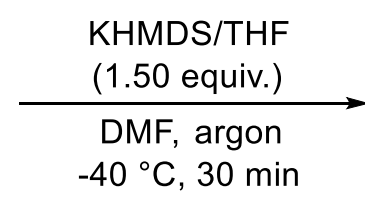

$-40^{\circ} \mathrm{C}, 30 \mathrm{~min}$

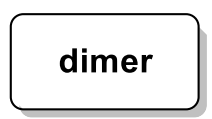

6

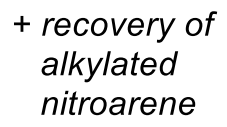

4

A $30 \mathrm{~mL}$ Schlenk flask was charged with alkylated alkylated nitroarene (4) (1.00 mmol) and flushed with argon. Dry DMF ( $3 \mathrm{~mL}$ ) was added, and after dissolution of 4 mixture was cooled to $-40{ }^{\circ} \mathrm{C}$. To the stirred reaction mixture KHMDS $(1.50 \mathrm{mmol}$, solution in THF*) was added rapidly in one portion. After 30 min reaction mixture was quenched with $\mathrm{NH}_{4} \mathrm{Cl}_{\mathrm{aq}}(5 \mathrm{~mL}, 10 \% \mathrm{w} / \mathrm{v})$. Mixture was transferred to separatory funnel, $\mathrm{NH}_{4} \mathrm{Cl}_{\mathrm{aq}}(50 \mathrm{~mL}, 10 \% \mathrm{w} / \mathrm{v})$ was added, mixture was extracted with ethyl acetate (3 $\times 50 \mathrm{~mL})$, combined organic phases were washed with $\mathrm{LiCl}_{\mathrm{aq}}(50 \mathrm{~mL}, 1.0 \mathrm{M}), \mathrm{H}_{2} \mathrm{O}(50 \mathrm{~mL})$, brine $(50$ $\mathrm{mL}$ ), and dried over anhydrous $\mathrm{MgSO}_{4}$. Mixture was filtered, evaporated and separated with column chromatography.

* - for concentrations of commercially-available solutions of KHMDS in THF used in this study see the General Information

For entries 1 and 2 crude reaction mixtures after quench were analyzed by ${ }^{1} \mathrm{H}$ NMR.

\begin{tabular}{|c|c|c|c|}
\hline entry & alkylated nitroarene & $\begin{array}{c}\text { alkylated nitroarene } \\
\text { recovery }\end{array}$ & dimer yield \\
\hline 1 & $\mathbf{4 v}$ & $34 \%^{\mathrm{a}}$ & $\mathbf{6 a}, 66 \%^{\mathrm{a}}$ \\
\hline 2 & $\mathbf{4 e}$ & $95 \%^{\mathrm{a}}$ & $\mathbf{6 d}, 5 \%^{\mathrm{a}}$ \\
\hline $3^{\mathrm{b}}$ & $\mathbf{4 a}$ & $36 \%$ & $\mathbf{6 c}, 39 \%$ \\
\hline $4^{\mathrm{b}}$ & $\mathbf{4 c}$ & $97 \%$ & - \\
\hline
\end{tabular}

$\mathrm{a}-{ }^{1} \mathrm{H}$ NMR conversions

$\mathrm{b}-1.00$ equiv. of KHMDS/THF was used 


\subsection{Experiment with radical scavenger (TEMPO)}

In order to exclude radical pathway of the alkylation reaction, experiment with 1.5 equiv. of TEMPO (2,2,6,6-Tetramethylpiperidine 1-oxyl) was carried out according to the General Procedure.

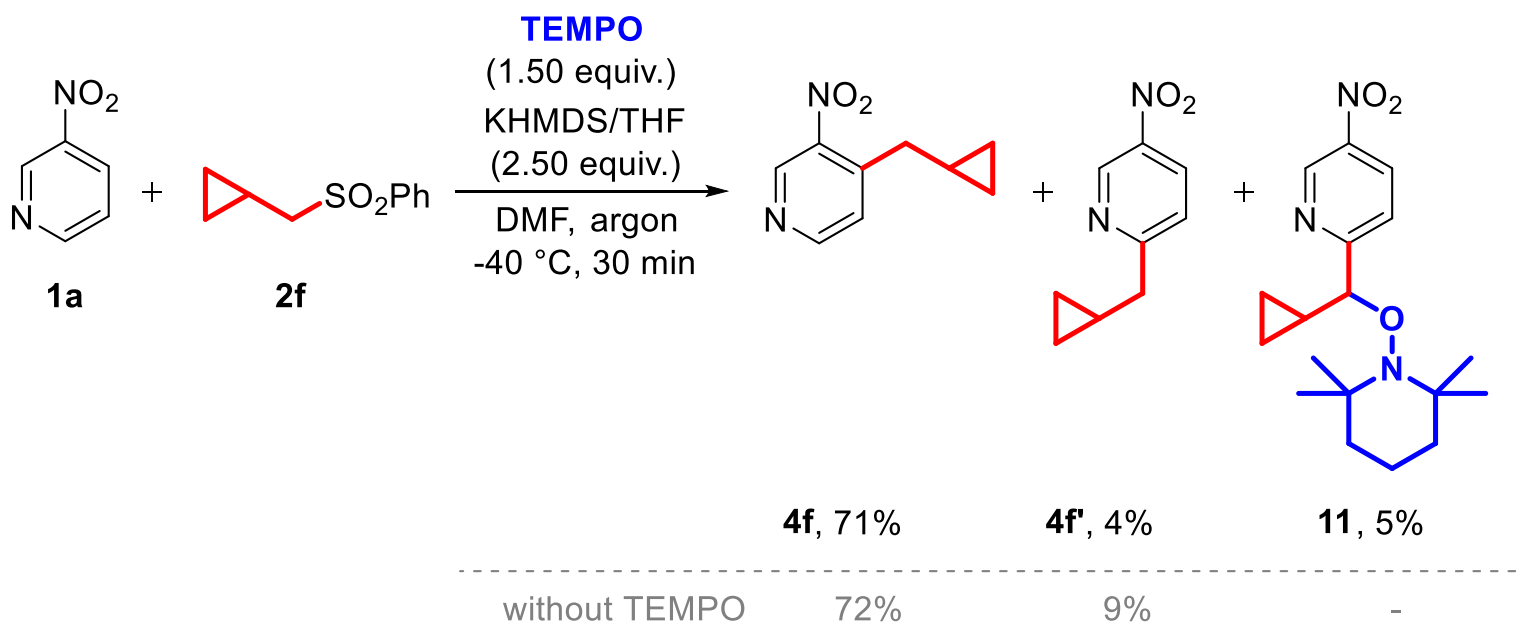

Yield of main product, 4-isobutyl-3-nitropyridine (4f) remained unchanged, compared to the reaction without TEMPO. Yield of 2-isobutyl-5-nitropyridine (4f') decreased by 5\% (from 9\% to 4\%), and 5\% of the corresponding adduct of minor isomer with TEMPO (11) was formed.<smiles>CC1(C)CCCC(C)(C)N1OC(c1ccc([N+](=O)[O-])cn1)C1CC1</smiles>

11

Yield:

$39 \mathrm{mg}(0.12 \mathrm{mmol}, \mathbf{5 \%})$ from $\mathbf{1 a}(248 \mathrm{mg}, 2.00 \mathrm{mmol})$

light yellow solid, mp $54.5-56.5^{\circ} \mathrm{C}$

eluent: toluene/ethyl acetate $50: 1$ to $1: 1$, then ethyl acetate

${ }^{1}$ H NMR (400 MHz, $\left.\mathbf{C D C l}_{3}\right): \delta=9.32(\mathrm{~d}, J=2.6 \mathrm{~Hz}, 1 \mathrm{H}), 8.44(\mathrm{dd}, J=$ $8.6,2.6 \mathrm{~Hz}, 1 \mathrm{H}), 7.62(\mathrm{~d}, J=8.6 \mathrm{~Hz}, 1 \mathrm{H}), 4.41(\mathrm{~d}, J=8.3 \mathrm{~Hz}, 1 \mathrm{H}), 1.62$ $0.90(\mathrm{~m}, 18 \mathrm{H}), 0.78-0.67(\mathrm{~m}, 1 \mathrm{H}), 0.67-0.54(\mathrm{~m}, 3 \mathrm{H}), 0.40-0.31(\mathrm{~m}$, $1 \mathrm{H}), 0.30-0.19(\mathrm{~m}, 1 \mathrm{H})$

${ }^{13} \mathbf{C}$ NMR (100 MHz, $\left.\mathbf{C D C l}_{3}\right): \delta=169.3,143.8,142.9,131.0,121.8,90.8$, 60.0, 40.2, 34.4, 33.8, 20.4, 20.1, 17.0, 16.9, 7.5, 1.8.

HRMS (ESI): $\mathrm{m} / \mathrm{z}[\mathrm{M}+\mathrm{H}]^{+}$calcd for $\mathrm{C}_{18} \mathrm{H}_{28} \mathrm{~N}_{3} \mathrm{O}_{3}:$ : 334.2125; found: 334.2121 . 


\section{One-pot alkylation-fluorination of nitroarenes}

Reaction was performed according to the General Procedure. After 30 min solution of Selectfluor in DMF was added and stirred for additional $60 \mathrm{~min}$, before quenching with $\mathrm{NH}_{4} \mathrm{Cl}_{\mathrm{aq}}{ }^{[22]}$

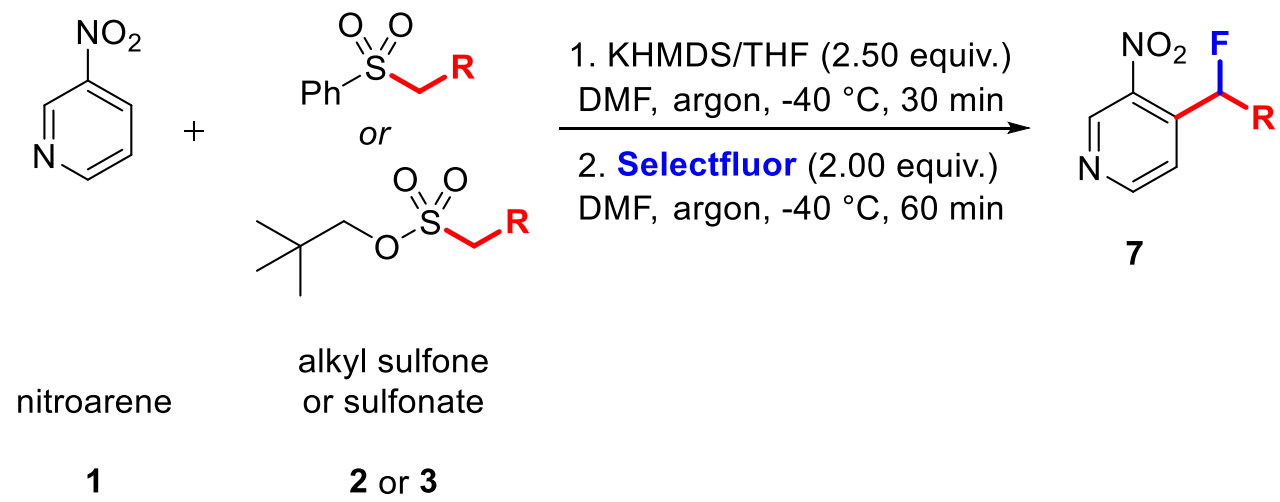

A $30 \mathrm{~mL}$ Schlenk flask was charged with nitroarene $(\mathbf{1}, 1.00 \mathrm{mmol})$, carbanion precursor $(\mathbf{2}$ or $\mathbf{3}$, $1.20 \mathrm{mmol})$, and flushed with argon. Dry DMF (3 mL) was added and after dissolution of substrates mixture was cooled to $-40{ }^{\circ} \mathrm{C}$. To the stirred reaction mixture KHMDS $(2.50 \mathrm{mmol}$, solution in THF*) was added rapidly in one portion. After $30 \mathrm{~min}$ solution of Selectfluor $(2.00 \mathrm{mmol})$ in dry DMF $(3 \mathrm{~mL})$ was slowly added, and after additional $1 \mathrm{~h}$ reaction mixture was quenched with $\mathrm{NH}_{4} \mathrm{Cl}_{\mathrm{aq}}(10 \mathrm{~mL}$, $10 \% \mathrm{w} / \mathrm{v})$. Mixture was transferred to separatory funnel, $\mathrm{NH}_{4} \mathrm{Cl}_{\mathrm{aq}}(50 \mathrm{~mL}, 10 \% \mathrm{w} / \mathrm{v})$ was added, mixture was extracted with ethyl acetate $(3 \times 50 \mathrm{~mL})$, combined organic phases were washed with $\mathrm{LiCl}_{\mathrm{aq}}(50 \mathrm{~mL}$, $1.0 \mathrm{M}), \mathrm{H}_{2} \mathrm{O}(50 \mathrm{~mL})$, brine $(50 \mathrm{~mL})$, and dried over anhydrous $\mathrm{MgSO}_{4}$. Mixture was filtered, evaporated and separated with column chromatography.

* - for concentrations of commercially-available solutions of KHMDS in THF used in this study see the General Information 
<smiles>CC(C)C(F)c1ccncc1[N+](=O)[O-]</smiles>

Yield:

$102 \mathrm{mg}(0.52 \mathrm{mmol}, \mathbf{5 0 \%})$ from $\mathbf{1 a}(129 \mathrm{mg}, 1.04 \mathrm{mmol})$ and $\mathbf{3 c}$

orange oil

eluent: toluene/ethyl acetate $100: 1$ to $1: 1$, then ethyl acetate

${ }^{1} \mathbf{H}$ NMR $\left(400 \mathrm{MHz}, \mathbf{C D C l}_{3}\right): \delta=9.23(\mathrm{~s}, 1 \mathrm{H}), 8.81(\mathrm{~d}, J=5.2 \mathrm{~Hz}, 1 \mathrm{H})$, $7.60(\mathrm{~d}, J=5.2 \mathrm{~Hz}, 1 \mathrm{H}), 6.09(\mathrm{dd}, J=49.0,3.2 \mathrm{~Hz}, 1 \mathrm{H}), 2.15-1.96(\mathrm{~m}$, $1 \mathrm{H}), 1.11(\mathrm{~d}, J=7.0 \mathrm{~Hz}, 3 \mathrm{H}), 0.81(\mathrm{dd}, J=7.0,1.0 \mathrm{~Hz}, 3 \mathrm{H})$.

${ }^{13}$ C NMR (100 MHz, $\left.\mathbf{C D C l}_{3}\right): \delta=153.7(\mathrm{~d}, J=2.1 \mathrm{~Hz}), 146.1(\mathrm{~d}, J=1.5$ $\mathrm{Hz}), 144.8(\mathrm{~d}, J=23.2 \mathrm{~Hz}), 128.7(\mathrm{~d}, J=70.6 \mathrm{~Hz}), 121.7(\mathrm{~d}, J=14.3 \mathrm{~Hz})$, $92.6(\mathrm{~d}, J=179.1 \mathrm{~Hz}), 33.1(\mathrm{~d}, J=21.8 \mathrm{~Hz}), 19.4(\mathrm{~d}, J=3.3 \mathrm{~Hz}), 14.9(\mathrm{~d}$, $J=6.1 \mathrm{~Hz})$.

${ }^{19}$ F NMR (376 MHz, $\left.\mathbf{C D C l}_{3}\right): \delta=-198.2(\mathrm{dd}, J=49.0,28.4 \mathrm{~Hz})$.

HRMS (ESI): $\mathrm{m} / \mathrm{z}[\mathrm{M}+\mathrm{H}]^{+}$calcd for $\mathrm{C}_{9} \mathrm{H}_{12} \mathrm{~N}_{2} \mathrm{O}_{2} \mathrm{~F}$ : 199.0883; found: 199.0889 .<smiles>CC(C)C(F)c1cc(Sc2ccccc2)ncc1[N+](=O)[O-]</smiles>

7b

Yield:

$343 \mathrm{mg}(1.12 \mathrm{mmol}, \mathbf{5 6 \%})$ from $\mathbf{1 d}(468 \mathrm{mg}, 2.02 \mathrm{mmol})$ and $\mathbf{3 c}$ yellow oil eluent: cyclohexane/toluene $5: 1$ to $1: 2$, then toluene, then toluene/ethyl acetate $50: 1$ to $5: 1$

${ }^{1}$ H NMR (400 MHz, CDCl $): \delta=9.24(\mathrm{~s}, 1 \mathrm{H}), 7.78-7.71(\mathrm{~m}, 2 \mathrm{H}), 7.68$ $-7.57(\mathrm{~m}, 3 \mathrm{H}), 7.24(\mathrm{~s}, 1 \mathrm{H}), 6.21(\mathrm{dd}, J=49.1,2.7 \mathrm{~Hz}, 1 \mathrm{H}), 2.26-2.06$ $(\mathrm{m}, 1 \mathrm{H}), 1.26(\mathrm{~d}, J=6.9 \mathrm{~Hz}, 3 \mathrm{H}), 0.88(\mathrm{~d}, J=6.9 \mathrm{~Hz}, 3 \mathrm{H})$.

${ }^{13}$ C NMR (100 MHz, $\left.\mathbf{C D C l}_{3}\right): \delta=169.3(\mathrm{~d}, J=2.2 \mathrm{~Hz}), 146.6(\mathrm{~d}, J=1.7$ $\mathrm{Hz}), 145.0(\mathrm{~d}, J=22.4 \mathrm{~Hz}), 138.9(\mathrm{~d}, J=4.7 \mathrm{~Hz}), 135.3,130.3,129.9$, $128.4,117.5(\mathrm{~d}, J=16.5 \mathrm{~Hz}), 92.6(\mathrm{~d}, J=180.0 \mathrm{~Hz}), 32.8(\mathrm{~d}, J=22.0 \mathrm{~Hz})$, $19.5(\mathrm{~d}, J=2.7 \mathrm{~Hz}), 14.5(\mathrm{~d}, J=6.3 \mathrm{~Hz})$.

${ }^{19}$ F NMR (376 MHz, $\left.\mathbf{C D C l}_{3}\right): \delta=-199.3(\mathrm{dd}, J=49.1,29.7 \mathrm{~Hz})$.

MS (EI): m/z (\%): $306\left(65,[\mathrm{M}]^{+}\right), 305\left(100,[\mathrm{M}-\mathrm{H}]^{+}\right), 217(29$, $\left.\left[\mathrm{M}-\mathrm{C}_{3} \mathrm{H}_{7} \mathrm{NO}_{2}\right]^{+}\right)$.

HRMS (EI): $\mathrm{m} / \mathrm{z}[\mathrm{M}]^{+}$calcd for $\mathrm{C}_{15} \mathrm{H}_{15} \mathrm{~N}_{2} \mathrm{O}_{2} \mathrm{FS}$ : 306.0838; found: 306.0824 . 
<smiles>CC(C)(C)C(F)c1ccncc1[N+](=O)[O-]</smiles>

Yield:

$218 \mathrm{mg}(1.03 \mathrm{mmol}, \mathbf{5 1 \%})$ from $\mathbf{1 a}(249 \mathrm{mg}, 2.01 \mathrm{mmol})$ and $\mathbf{2 d}$

yellowish oil

eluent: toluene, then toluene/ethyl acetate 50:1 to 20:1, then ethyl acetate

${ }^{1} \mathbf{H}$ NMR $\left(400 \mathrm{MHz}, \mathbf{C D C l}_{3}\right): \delta=9.13(\mathrm{~s}, 1 \mathrm{H}), 8.82(\mathrm{~d}, J=5.2 \mathrm{~Hz}, 1 \mathrm{H})$, $7.59(\mathrm{~d}, J=5.2 \mathrm{~Hz}, 1 \mathrm{H}), 6.23(\mathrm{~d}, J=46.7 \mathrm{~Hz}, 1 \mathrm{H}), 0.91(\mathrm{~d}, J=1.5 \mathrm{~Hz}$, 9H).

${ }^{13}$ C NMR (100 MHz, $\left.\mathbf{C D C l}_{3}\right): \delta=153.0(\mathrm{~d}, J=1.5 \mathrm{~Hz}), 145.5(\mathrm{~d}, J=1.3$ $\mathrm{Hz}), 144.10,141.7(\mathrm{~d}, J=24.4 \mathrm{~Hz}), 122.8(\mathrm{~d}, J=12.5 \mathrm{~Hz}), 93.3(\mathrm{~d}, J=$ $179.6 \mathrm{~Hz}), 36.8(\mathrm{~d}, J=21.1 \mathrm{~Hz}), 24.8(\mathrm{~d}, J=4.6 \mathrm{~Hz})$.

${ }^{19}$ F NMR (376 MHz, $\left.\mathbf{C D C l}_{3}\right): \delta=-189.5(\mathrm{~d}, J=46.7 \mathrm{~Hz})$.

HRMS (ESI): $\mathrm{m} / \mathrm{z}[\mathrm{M}+\mathrm{H}]^{+}$calcd for $\mathrm{C}_{10} \mathrm{H}_{14} \mathrm{~N}_{2} \mathrm{O}_{2} \mathrm{~F}$ : 213.1039; found: 213.1037.<smiles>CC(C)(C)C(F)c1cc(Sc2ccccc2)ncc1[N+](=O)[O-]</smiles>

7d

Yield:

$390 \mathrm{mg}$ (1.22 mmol, 61\%) form $\mathbf{1 d}(466 \mathrm{mg}, 2.01 \mathrm{mmol})$ and $\mathbf{2 d}$

light yellow solid, mp $46.0-48.0{ }^{\circ} \mathrm{C}$

eluent: cyclohexane/toluene $2: 1$ to $1: 2$, then toluene, then toluene/ethyl acetate $50: 1$ to $20: 1$

${ }^{1}$ H NMR (400 MHz, CDCl $): \delta=8.96(\mathrm{~s}, 1 \mathrm{H}), 7.62-7.55(\mathrm{~m}, 2 \mathrm{H}), 7.52$ $-7.43(\mathrm{~m}, 3 \mathrm{H}), 6.98(\mathrm{~s}, 1 \mathrm{H}), 6.20(\mathrm{~d}, J=46.9 \mathrm{~Hz}, 1 \mathrm{H}), 0.80(\mathrm{~d}, J=1.7 \mathrm{~Hz}$, $9 \mathrm{H})$.

${ }^{13}$ C NMR (100 MHz, $\left.\mathbf{C D C l}_{3}\right): \delta=168.5(\mathrm{~d}, J=1.4 \mathrm{~Hz}), 145.9(\mathrm{~d}, J=1.4$ $\mathrm{Hz}), 142.1(\mathrm{~d}, J=23.5 \mathrm{~Hz}), 140.6(\mathrm{~d}, J=4.9 \mathrm{~Hz}), 135.4,130.3,130.0$, $128.5,118.7(\mathrm{~d}, J=13.7 \mathrm{~Hz}), 93.1(\mathrm{~d}, J=179.9 \mathrm{~Hz}), 36.8(\mathrm{~d}, J=21.2 \mathrm{~Hz})$, $24.7(\mathrm{~d}, J=4.6 \mathrm{~Hz})$.

${ }^{19}$ F NMR (376 MHz, CDCl 3$): ~ \delta=-189.0(\mathrm{~d}, J=47.2 \mathrm{~Hz})$.

MS (EI): $\mathrm{m} / \mathrm{z}(\%)=321\left(29,[\mathrm{M}+\mathrm{H}]^{+}\right), 320\left(82,[\mathrm{M}]^{+}\right), 319\left(100,[\mathrm{M}-\mathrm{H}]^{+}\right)$, $247\left(32,\left[\mathrm{M}-\mathrm{OC}_{4} \mathrm{H}_{9}\right]^{+}\right), 186\left(47,\left[\mathrm{M}-\mathrm{C}_{5} \mathrm{H}_{9} \mathrm{FNO}_{2}\right]^{+}\right), 58\left(90,\left[\mathrm{C}_{4} \mathrm{H}_{10}\right]^{+}\right)$.

HRMS (EI): $\mathrm{m} / \mathrm{z}$ [M] ${ }^{+}$calcd for $\mathrm{C}_{16} \mathrm{H}_{17} \mathrm{~N}_{2} \mathrm{O}_{2} \mathrm{FS}$ : 320.0995; found: 320.0981 . 


\section{Postsynthetic transformations of the alkylated pyridines}

\subsection{Reduction of the $\mathrm{NO}_{2}$ group with subsequent acylation}

Reduction of the $\mathrm{NO}_{2}$ group was performed using $\mathrm{H}_{2}$ with $\mathrm{Pd} / \mathrm{C}$ in $\mathrm{MeOH}$. Crude product was acylated with $\mathrm{Ac}_{2} \mathrm{O}$.

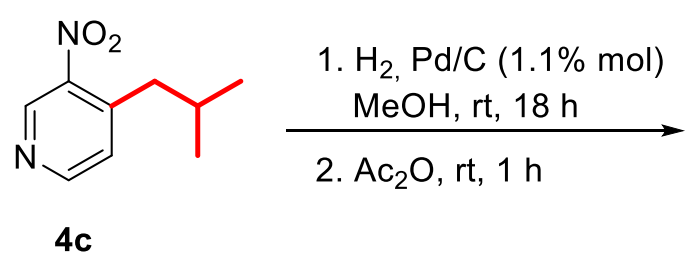<smiles>CC(=O)Nc1cnccc1CC(C)C</smiles>

A $25 \mathrm{~mL}$ round-bottom flask was charged with 4-isobutyl-3-nitropyridine $(\mathbf{4 c}, 181 \mathrm{mg}, 1.00 \mathrm{mmol}$, 1.00 equiv.), $\mathrm{Pd} / \mathrm{C}$ (12 mg, $10 \% \mathrm{w} / \mathrm{w}, 0.01 \mathrm{mmol}, 1.1 \mathrm{~mol} \%$ ), evacuated, and supplied with $\mathrm{H}_{2}$ balloon. $\mathrm{MeOH}(4 \mathrm{~mL})$ was added, and reaction was stirred at $1000 \mathrm{rpm}$. After $18 \mathrm{~h}$ reaction mixture was filtered through a pad of Celite and concentrated. Crude product was placed in $25 \mathrm{~mL}$ round-bottom flask, acetic anhydride $(4 \mathrm{~mL})$ was added, and reaction mixture was stirred for $1 \mathrm{~h}$. Then, reaction mixture was poured on $\mathrm{H}_{2} \mathrm{O}(50 \mathrm{~mL})$ cooled in ice/water bath $\left(2-3{ }^{\circ} \mathrm{C}\right)$, and $\mathrm{K}_{2} \mathrm{CO}_{3}(12.5 \mathrm{~g})$ was added portionwise to $\mathrm{pH}$ ca. 10. Mixture was transferred to separatory funnel, extracted with dichloromethane $(3 \times 50 \mathrm{~mL})$, combined organic phases were washed with brine $(50 \mathrm{~mL})$, and dried over anhydrous $\mathrm{MgSO}_{4}$. Mixture was filtered, evaporated and dried under vacuum yielding $\mathbf{8}(190 \mathrm{mg}, 0.99 \mathrm{mmol}, \mathbf{9 8 \%})$ as an off-white solid.<smiles>CC(=O)Nc1cnccc1CC(C)C</smiles>

\section{8}

Yield:

$189.7 \mathrm{mg}(0.987 \mathrm{mmol}, \mathbf{9 8 \%})$ from $\mathbf{4 c}(180.8 \mathrm{mg}, 1.003 \mathrm{mmol})$

off-white solid, $\mathbf{m p} 97.0-99.0^{\circ} \mathrm{C}$

${ }^{1}$ H NMR (400 MHz, CDCl 3 ): $\delta=8.64(\mathrm{~s}, 1 \mathrm{H}), 8.54(\mathrm{~s}, 1 \mathrm{H}), 8.18(\mathrm{~d}, J=$ $5.0 \mathrm{~Hz}, 1 \mathrm{H}), 7.00(\mathrm{~d}, J=5.0 \mathrm{~Hz}, 1 \mathrm{H}), 2.35(\mathrm{~d}, J=7.2 \mathrm{~Hz}, 2 \mathrm{H}), 2.05(\mathrm{~s}$, $3 \mathrm{H}), 1.85-1.72(\mathrm{~m}, 1 \mathrm{H}), 0.79(\mathrm{~d}, J=6.7 \mathrm{~Hz}, 6 \mathrm{H})$.

${ }^{13}$ C NMR (100 MHz, CDCl $): ~ \delta=169.5,147.0,146.0,144.7,132.8,124.9$, $39.7,28.3,23.3,22.2$.

MS (EI): m/z (\%): $192\left(75,[\mathrm{M}]^{+}\right), 177\left(29,\left[\mathrm{M}-\mathrm{CH}_{3}\right]^{+}\right), 150(100$, $\left.\left[\mathrm{M}-\mathrm{C}_{3} \mathrm{H}_{6}\right]^{+}\right), 135\left(56,\left[\mathrm{M}-\mathrm{C}_{4} \mathrm{H}_{9}\right]^{+}\right), 107\left(94,\left[\mathrm{M}-\mathrm{C}_{3} \mathrm{H}_{6}-\mathrm{CH}_{3} \mathrm{CO}\right]^{+}\right)$.

HRMS (EI): $\mathrm{m} / \mathrm{z}$ [M] $]^{+}$calcd for $\mathrm{C}_{11} \mathrm{H}_{16} \mathrm{~N}_{2} \mathrm{O}: 192.1263$; found: 192.1262 . 


\subsection{Synthesis of azaindole under Bartoli conditions}

Transformation of $\mathbf{( 4 q )}$ into 4-azaindole (9) was performed according to the literature procedure. ${ }^{[23]}$

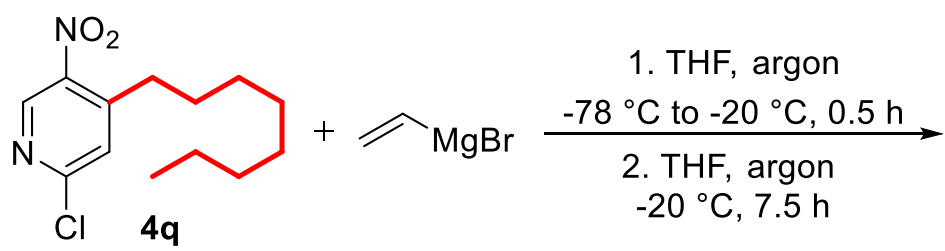<smiles>CCCCCCc1cc(Cl)nc2cc[nH]c12</smiles>

A $30 \mathrm{~mL}$ Schlenk flask was charged with 2-chloro-4-octyl-5-nitropyridine (4q) $(240 \mathrm{mg}, 0.89 \mathrm{mmol}$, 1.00 equiv.), and flushed with argon. Dry THF $(5.5 \mathrm{~mL})$ was added and after dissolution of substrate mixture was cooled to $-78{ }^{\circ} \mathrm{C}$. To the stirred reaction mixture vinylmagnesium bromide $(2.82 \mathrm{~mL}, 2.82$ mmol, $1.0 \mathrm{M}$ in THF, 3.18 equiv.) was added slowly (ca. $15 \mathrm{~s}$ ), and then mixture was slowly warmed to $-20{ }^{\circ} \mathrm{C}$ over $30 \mathrm{~min}$. Mixture was stirred for $7.5 \mathrm{~h}$ at $-20{ }^{\circ} \mathrm{C}$, and then quenched with $\mathrm{NH}_{4} \mathrm{Cl}_{\mathrm{aq}}(5 \mathrm{~mL}$, $10 \% \mathrm{w} / \mathrm{v})$. Mixture was transferred to separatory funnel, $\mathrm{NH}_{4} \mathrm{Cl}_{\mathrm{aq}}(50 \mathrm{~mL}, 10 \% \mathrm{w} / \mathrm{v})$ was added, mixture was extracted with ethyl acetate $(3 \times 50 \mathrm{~mL})$, combined organic phases were washed with $\mathrm{H}_{2} \mathrm{O}(50 \mathrm{~mL})$, brine $(50 \mathrm{~mL})$, and dried over anhydrous $\mathrm{MgSO}_{4}$. Mixture was filtered, evaporated and separated with column chromatography yielding $\mathbf{9}(87 \mathrm{mg}, 0.33 \mathrm{mmol}, \mathbf{3 7 \%})$ as an off-white solid.<smiles>CCCCCCCc1cc(Cl)nc2cc[nH]c12</smiles>

9

Yield: $87 \mathrm{mg}(0.33 \mathrm{mmol}, \mathbf{3 7 \%})$ from $\mathbf{4 q}(240 \mathrm{mg}, 0.89 \mathrm{mmol})$

off-white solid, mp $98.5-101.5^{\circ} \mathrm{C}$

eluent: cyclohexane/ethyl acetate 20:1 to $1: 2$

${ }^{1}$ H NMR (400 MHz, CDCl 3 ): $\delta=9.58($ br s, $1 \mathrm{H}), 7.44(\mathrm{t}, J=3.2 \mathrm{~Hz}, 1 \mathrm{H})$, $6.95(\mathrm{~s}, 1 \mathrm{H}), 6.59(\mathrm{dd}, J=3.2,2.0 \mathrm{~Hz}, 1 \mathrm{H}), 2.85-2.78(\mathrm{~m}, 2 \mathrm{H}), 1.75-$ $1.62(\mathrm{~m}, 2 \mathrm{H}), 1.32-1.13(\mathrm{~m}, 10 \mathrm{H}), 0.84(\mathrm{t}, J=7.0 \mathrm{~Hz}, 3 \mathrm{H})$.

${ }^{13}$ C NMR (100 MHz, CDCl $): ~ \delta=145.2,144.2,137.6,128.6,127.3,116.3$, $102.8,31.8,30.9,29.4,29.3,29.1,29.0,22.6,14.1$.

MS (EI): m/z (\%): $264\left(34,[\mathrm{M}]^{+}\right), 166\left(100,\left[\mathrm{M}-\mathrm{C}_{7} \mathrm{H}_{14}\right]^{+}\right)$,

HRMS (EI): $\mathrm{m} / \mathrm{z}$ [M] ${ }^{+}$calcd for $\mathrm{C}_{15} \mathrm{H}_{21} \mathrm{~N}_{2}{ }^{35} \mathrm{Cl}$ : 264.1393; found: 264.1399 . 


\section{References}

[1] D. Antoniak, M. Barbasiewicz, Org. Lett. 2019, 21, 9320-9325.

[2] P. K. Shyam, H. Y. Jang, J. Org. Chem. 2017, 82, 1761-1767.

[3] I. T. Phan, G. J. Gilbert, G. W. O’Neil, Synlett 2015, 26, 1867-1871.

[4] D. Nawrot, M. Kolenič, J. Kuneš, F. Kostelansky, M. Miletin, V. Novakova, P. Zimcik, Tetrahedron 2018, 74, 594-599.

[5] H. G. Viehe, S. Y. Delavarenne, Chem. Ber. 1970, 103, 1216-1224.

[6] W. E. Parham, L. D. Edwards, J. Org. Chem. 1968, 33, 4150-4154.

[7] B. Górski, D. Basiak, Ł. Grzesiński, M. Barbasiewicz, Org. Biomol. Chem. 2019, 17, 76607663.

[8] X. Marset, G. Guillena, D. J. Ramón, Chem. Eur. J. 2017, 23, 10522-10526.

[9] Y. G. Borisova, G. Z. Raskildina, S. S. Zlotskii, Russ. J. Gen. Chem. 2016, 86, 1954-1956.

[10] S. Mannathan, C. H. Cheng, Chem. Eur. J. 2012, 18, 11771-11777.

[11] B. Y. Hao, J. Q. Liu, W. H. Zhang, X. Z. Chen, Synthesis 2011, 1208-1212.

[12] N. Margraf, G. Manolikakes, J. Org. Chem. 2015, 80, 2582-2600.

[13] B. Górski, A. Talko, T. Basak, M. Barbasiewicz, Org. Lett. 2017, 19, 1756-1759.

[14] W. E. Truce, D. J. Vrencur, J. Org. Chem. 1970, 35, 1226-1227.

[15] B. Sreedhar, P. Surendra Reddy, M. Amarnath Reddy, Synthesis 2009, 1732-1738.

[16] H. C. Winter, F. E. Reinhart, J. Am. Chem. Soc. 1940, 62, 3508-3511.

[17] T. Kuramochi, A. Kakefuda, H. Yamada, I. Sato, T. Taguchi, S. Sakamoto, Bioorg. Med. Chem. 2004, 12, 5039-5056.

[18] K. R. Reddy, C. U. Maheswari, M. Venkateshwar, M. L. Kantam, Adv. Synth. Catal. 2009, 351, 93-96.

[19] P. J. Amal Joseph, S. Priyadarshini, M. Lakshmi Kantam, H. Maheswaran, Tetrahedron Lett. 2012, 53, 1511-1513.

[20] D. H. G. Crout, J. R. Penton, K. Schofield, J. Chem. Soc. B 1971, 1254-1256.

[21] A. R. Katritzky, E. F. V. Scriven, S. Majumder, R. G. Akhmedova, A. V. Vakulenko, N. G. Akhmedov, R. Murugan, K. A. Abboud, Org. Biomol. Chem. 2005, 3, 538-541.

[22] F. Y. Al-Mkhaizim, M. F. Greaney, Synlett 2020, 1094-1096.

[23] Z. Zhang, Z.Yang, N. A. Meanwell, J. F. Kadow, T. Wang, J. Org. Chem. 2002, 67, 23452347. 
8. Reproductions of NMR spectra
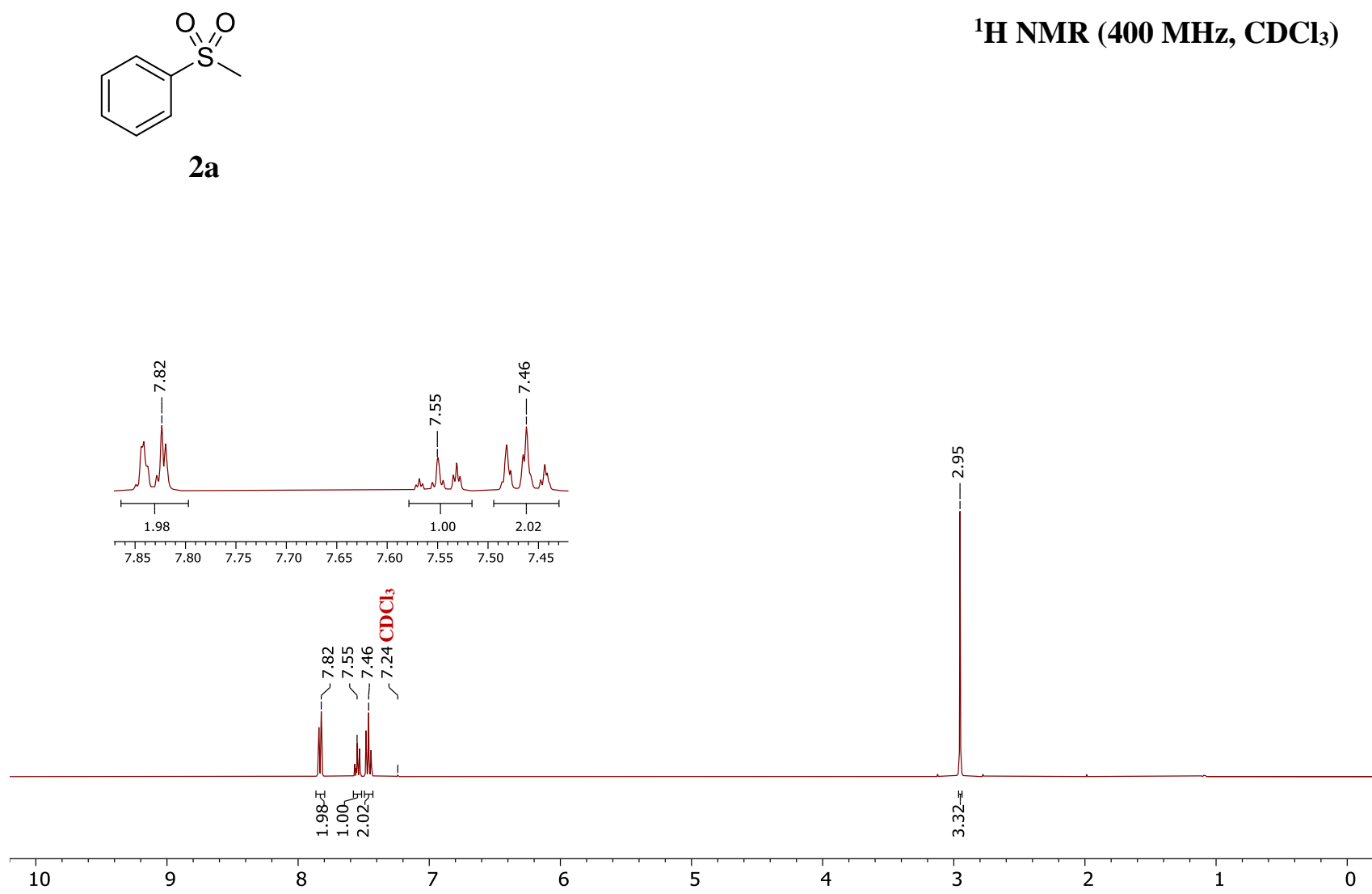

${ }^{13} \mathrm{C}$ NMR (100 MHz, $\left.\mathrm{CDCl}_{3}\right)$

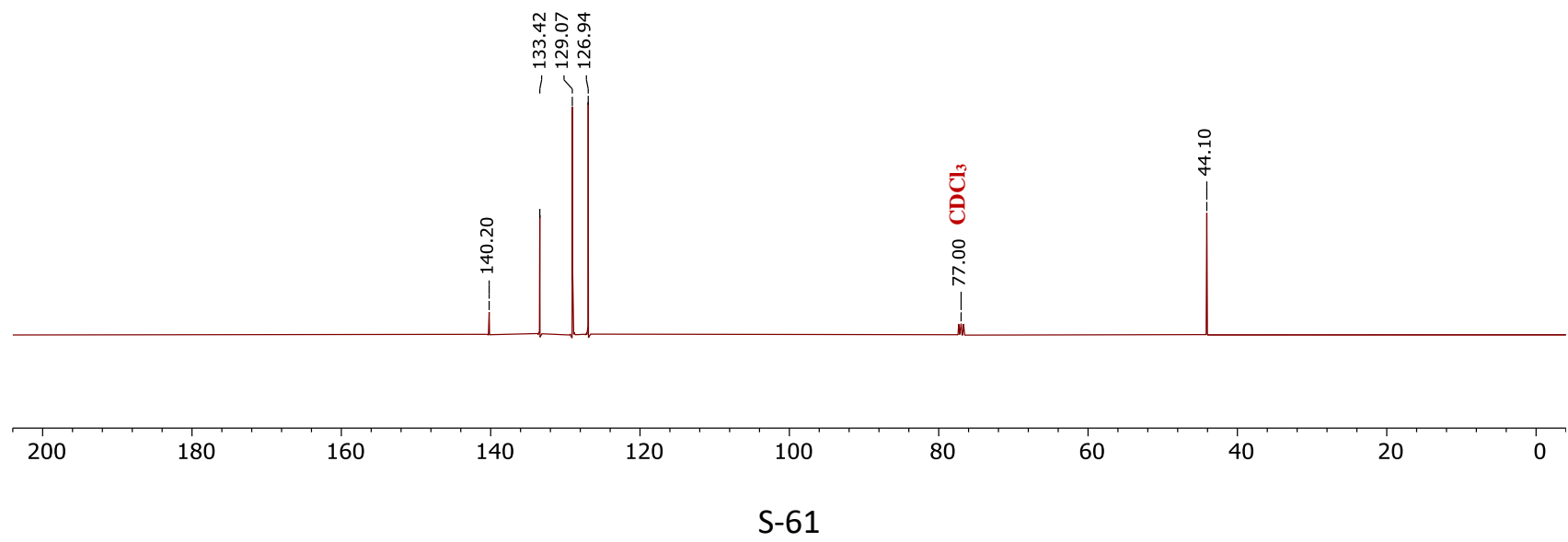


<smiles>CCS(=O)(=O)c1ccccc1</smiles>

$2 b$

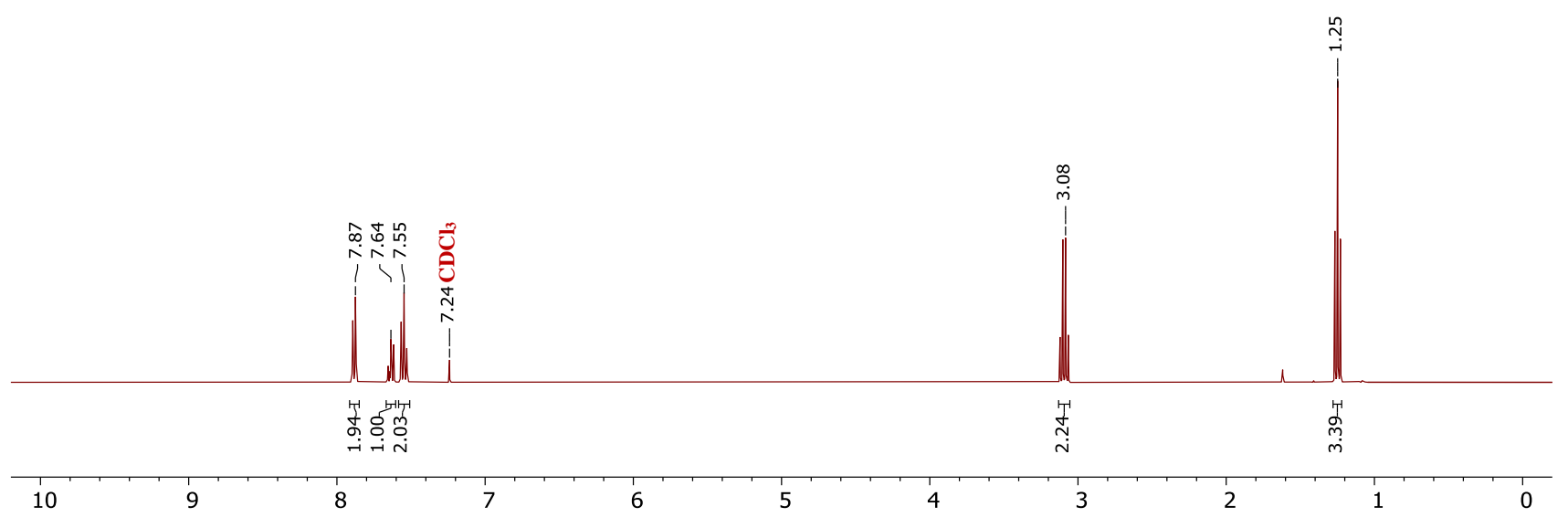

${ }^{13} \mathrm{C}$ NMR (100 MHz, $\left.\mathrm{CDCl}_{3}\right)$

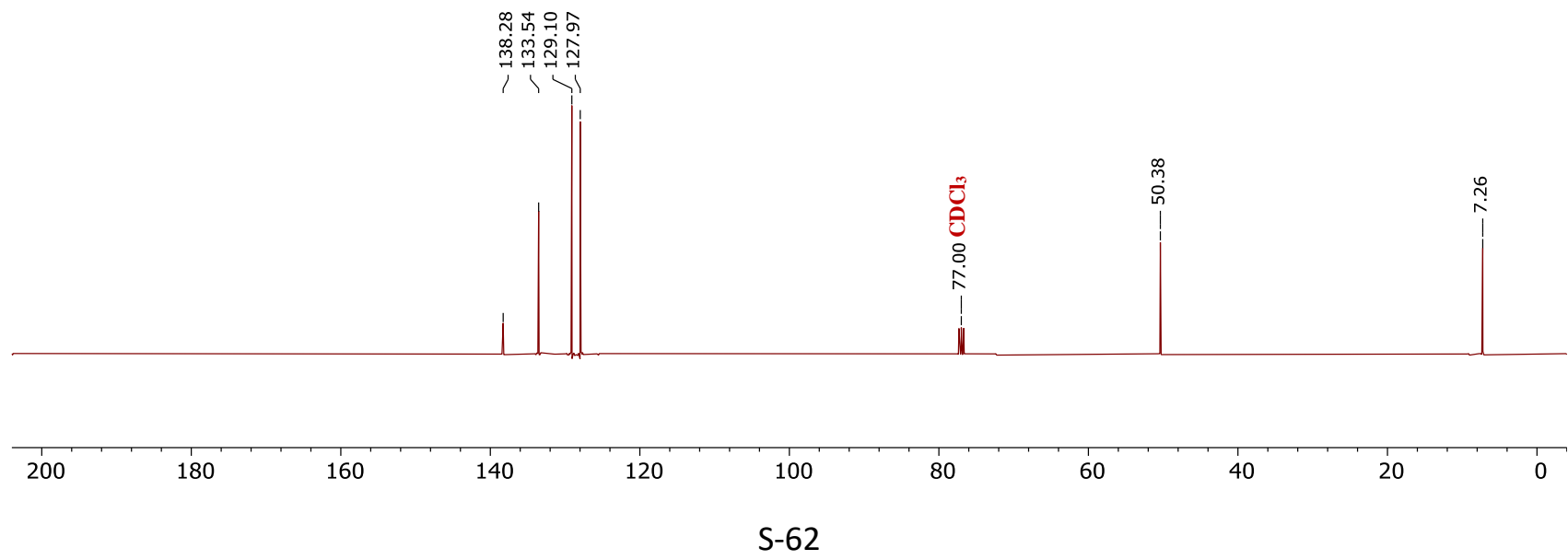




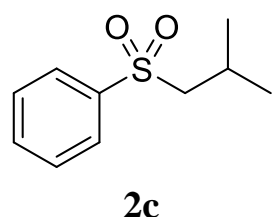

${ }^{1} \mathrm{H}$ NMR (400 MHz, $\left.\mathrm{CDCl}_{3}\right)$

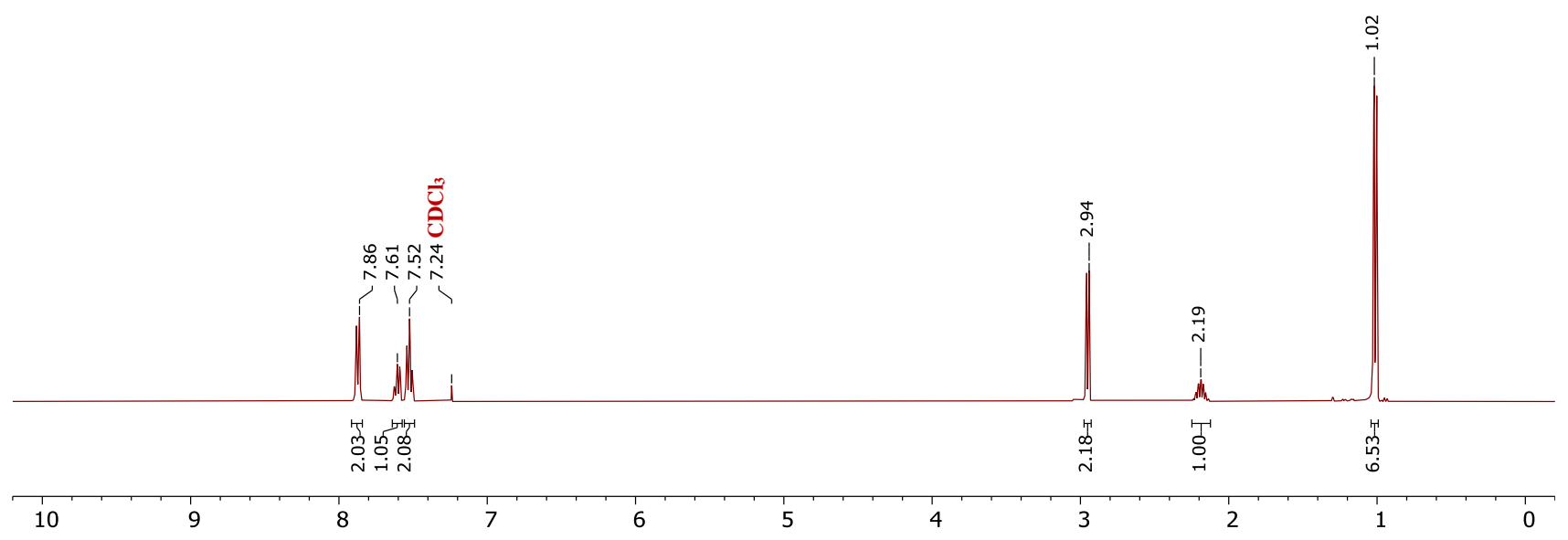

${ }^{13} \mathrm{C}$ NMR (100 MHz, $\left.\mathrm{CDCl}_{3}\right)$

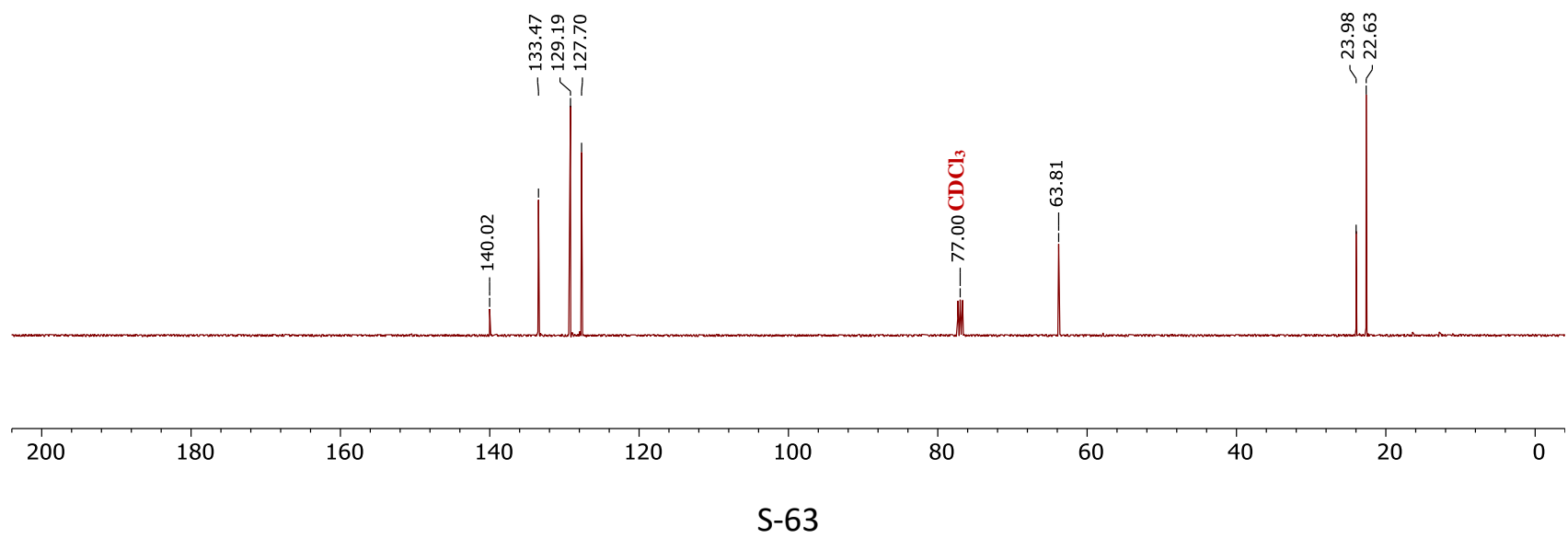


<smiles>CC(C)(C)CS(=O)(=O)c1ccccc1</smiles>

2d

\section{${ }^{1} \mathrm{H}$ NMR (400 MHz, $\mathrm{CDCl}_{3}$ )}

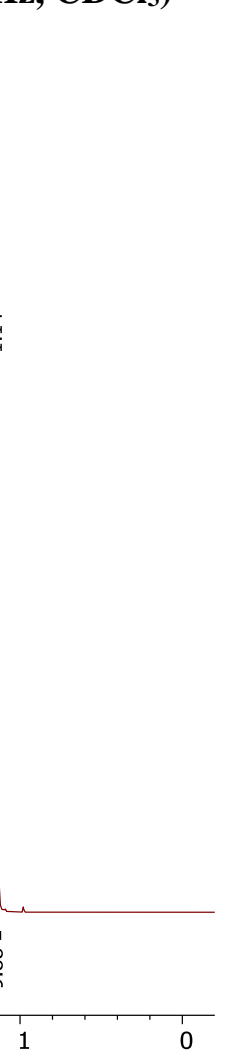

${ }^{13} \mathrm{C}$ NMR (100 MHz, $\left.\mathrm{CDCl}_{3}\right)$

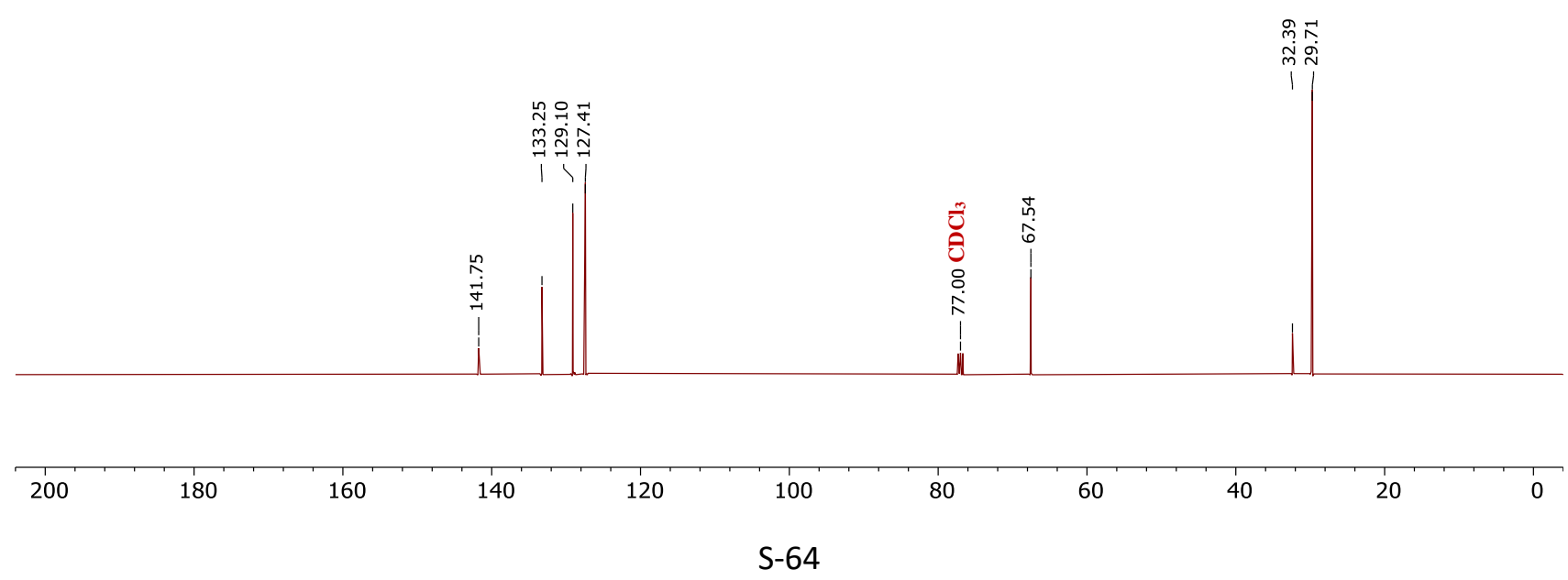




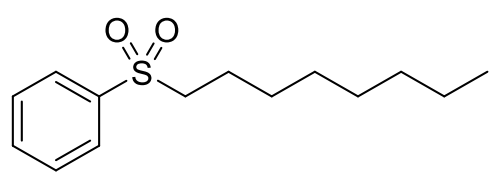

${ }^{1} \mathrm{H}$ NMR (400 MHz, $\mathrm{CDCl}_{3}$ )

$2 e$

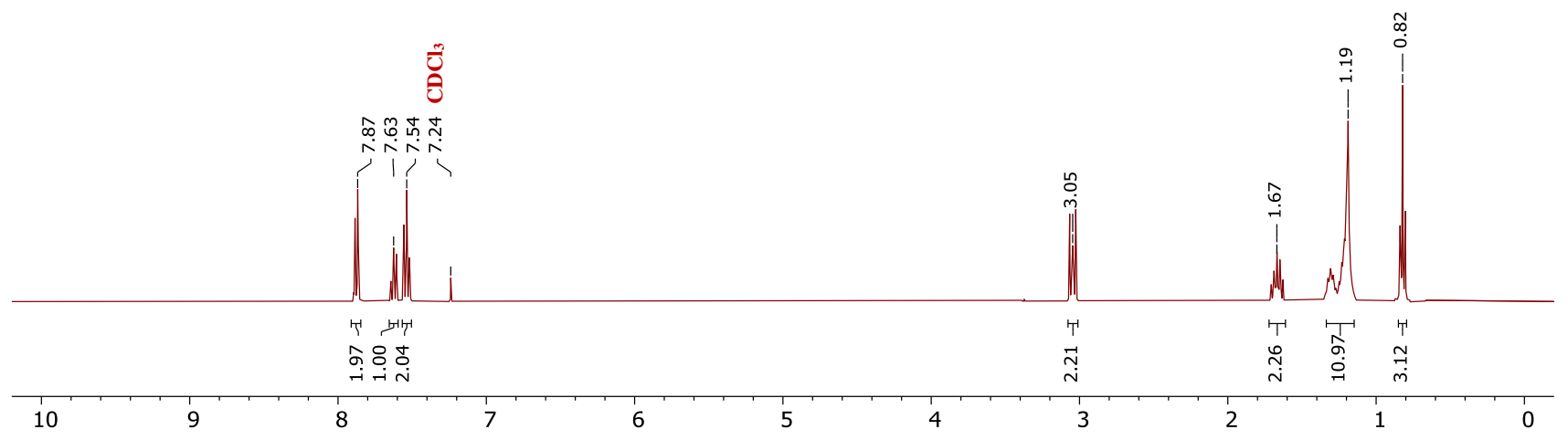

${ }^{13} \mathrm{C}$ NMR (100 MHz, $\left.\mathrm{CDCl}_{3}\right)$

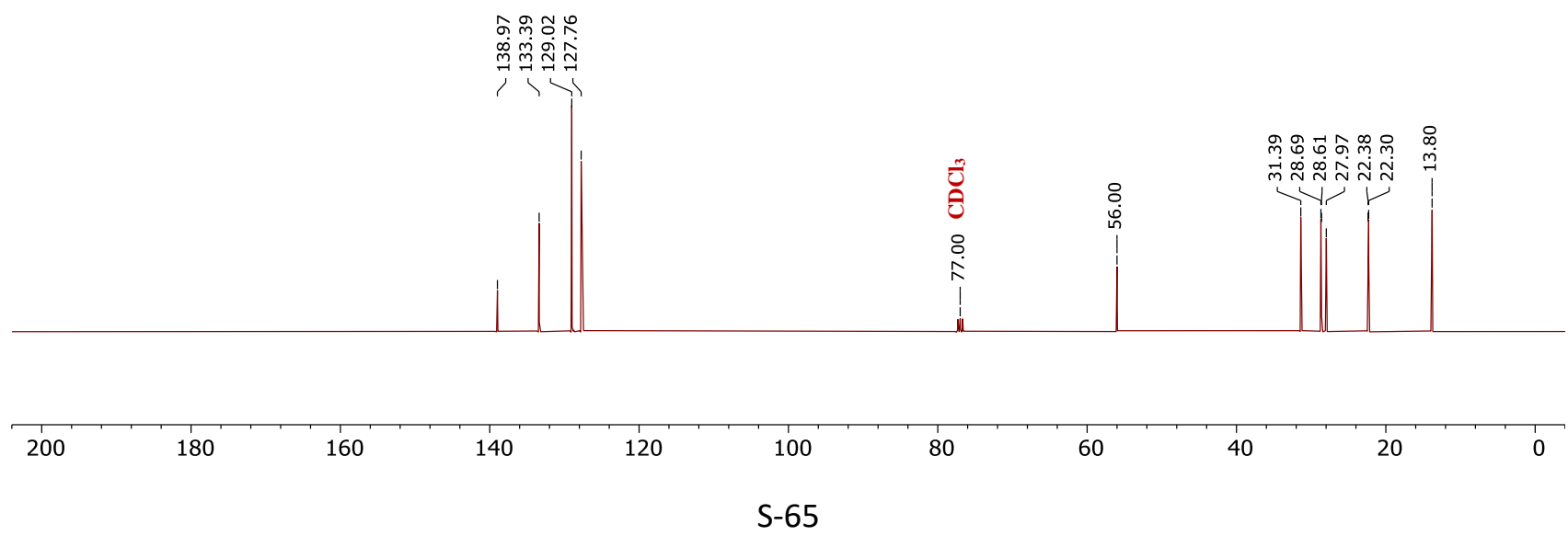




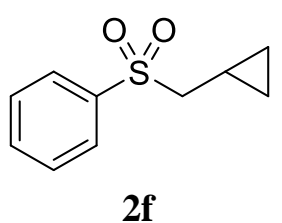

${ }^{1} \mathrm{H}$ NMR (400 MHz, $\mathrm{CDCl}_{3}$ )
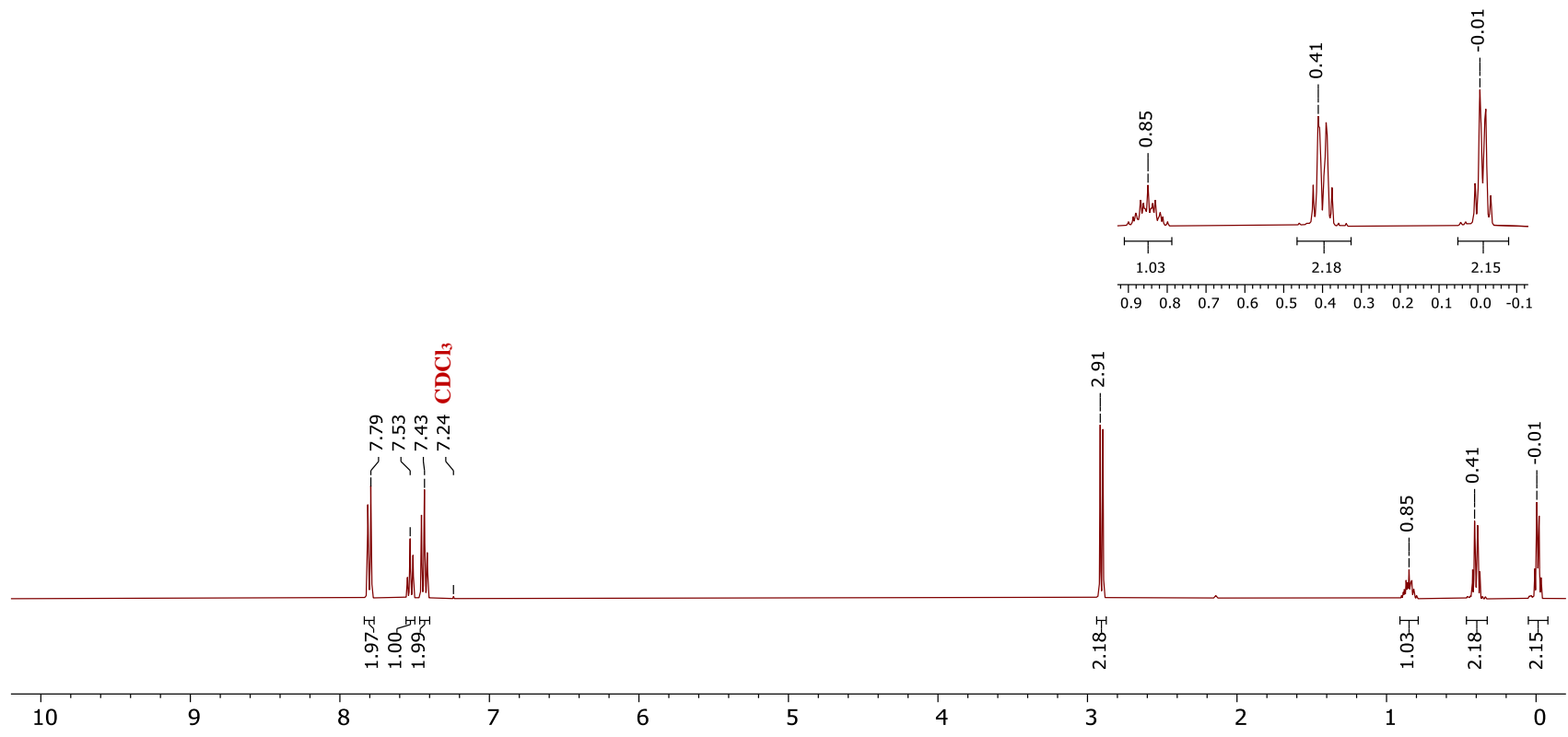

${ }^{13} \mathrm{C}$ NMR (100 MHz, $\left.\mathrm{CDCl}_{3}\right)$

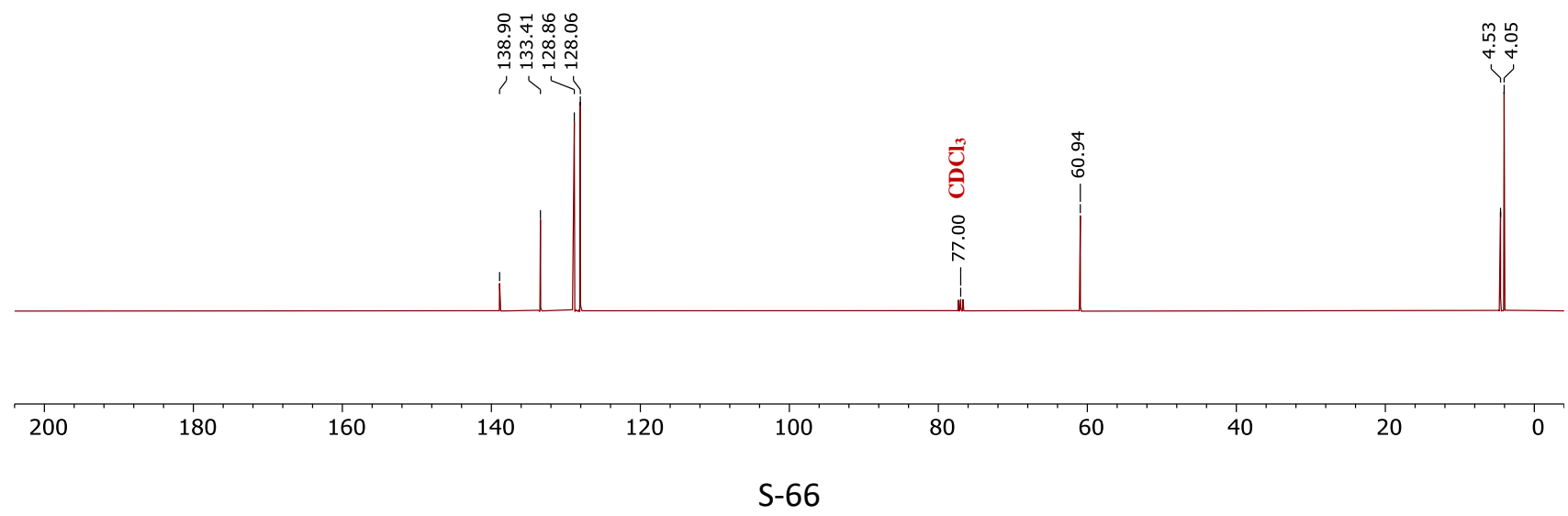




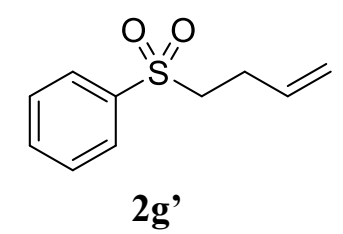

${ }^{1} \mathrm{H}$ NMR (400 MHz, $\mathrm{CDCl}_{3}$ )

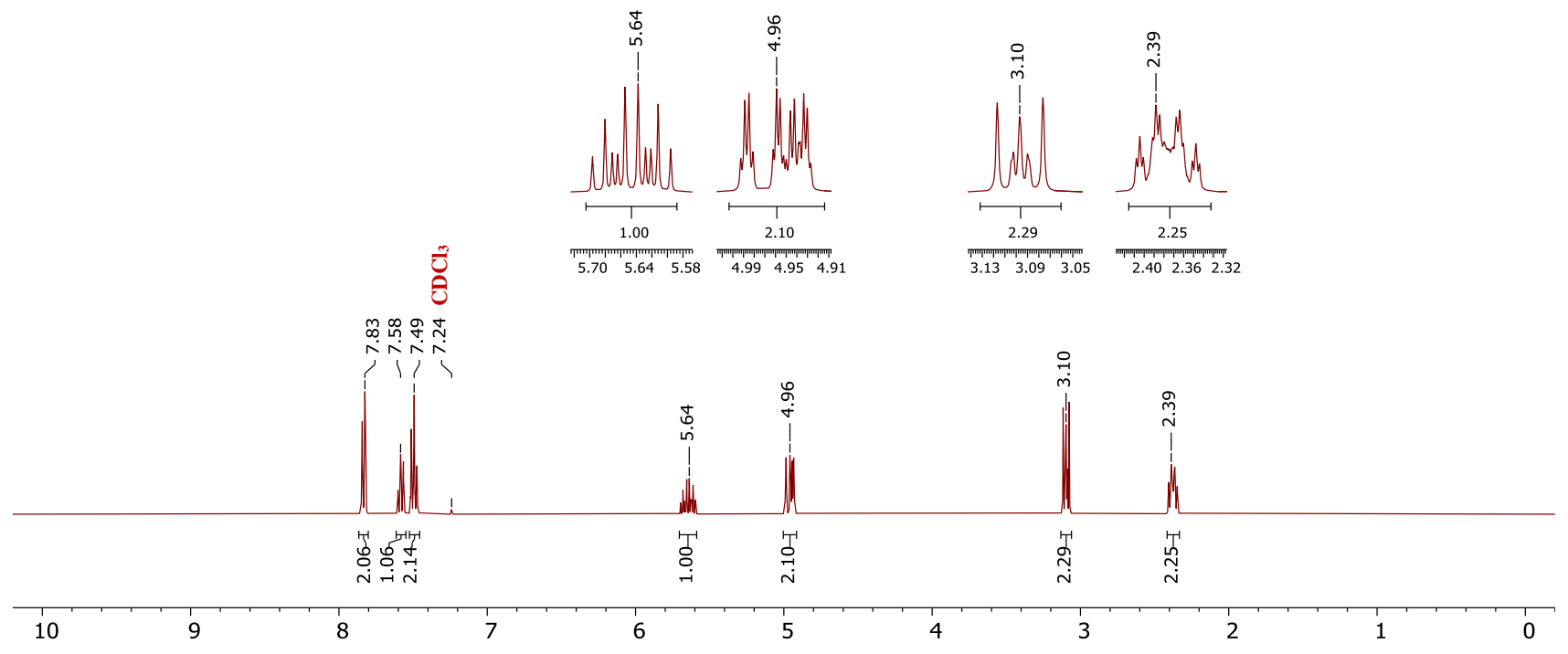

${ }^{13} \mathrm{C}$ NMR (100 MHz, $\left.\mathrm{CDCl}_{3}\right)$

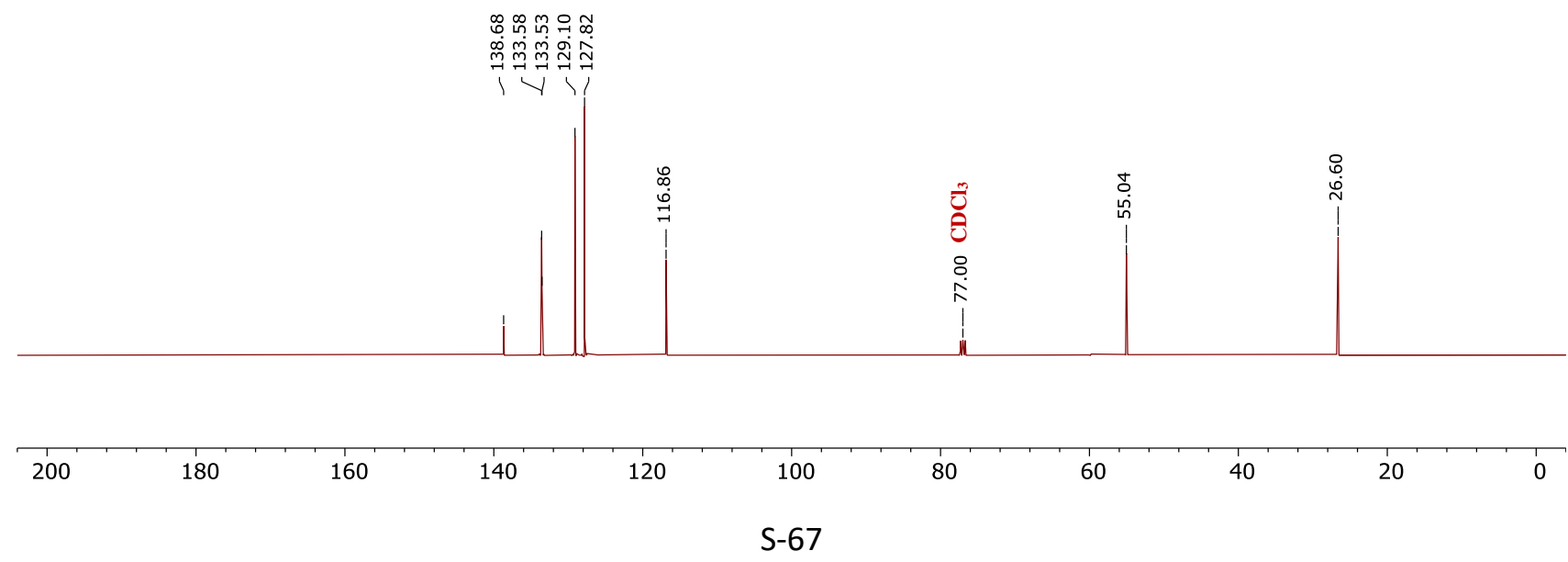


<smiles>O=S(=O)(CCC1CC1(Cl)Cl)c1ccccc1</smiles>

$2 \mathrm{~g}$
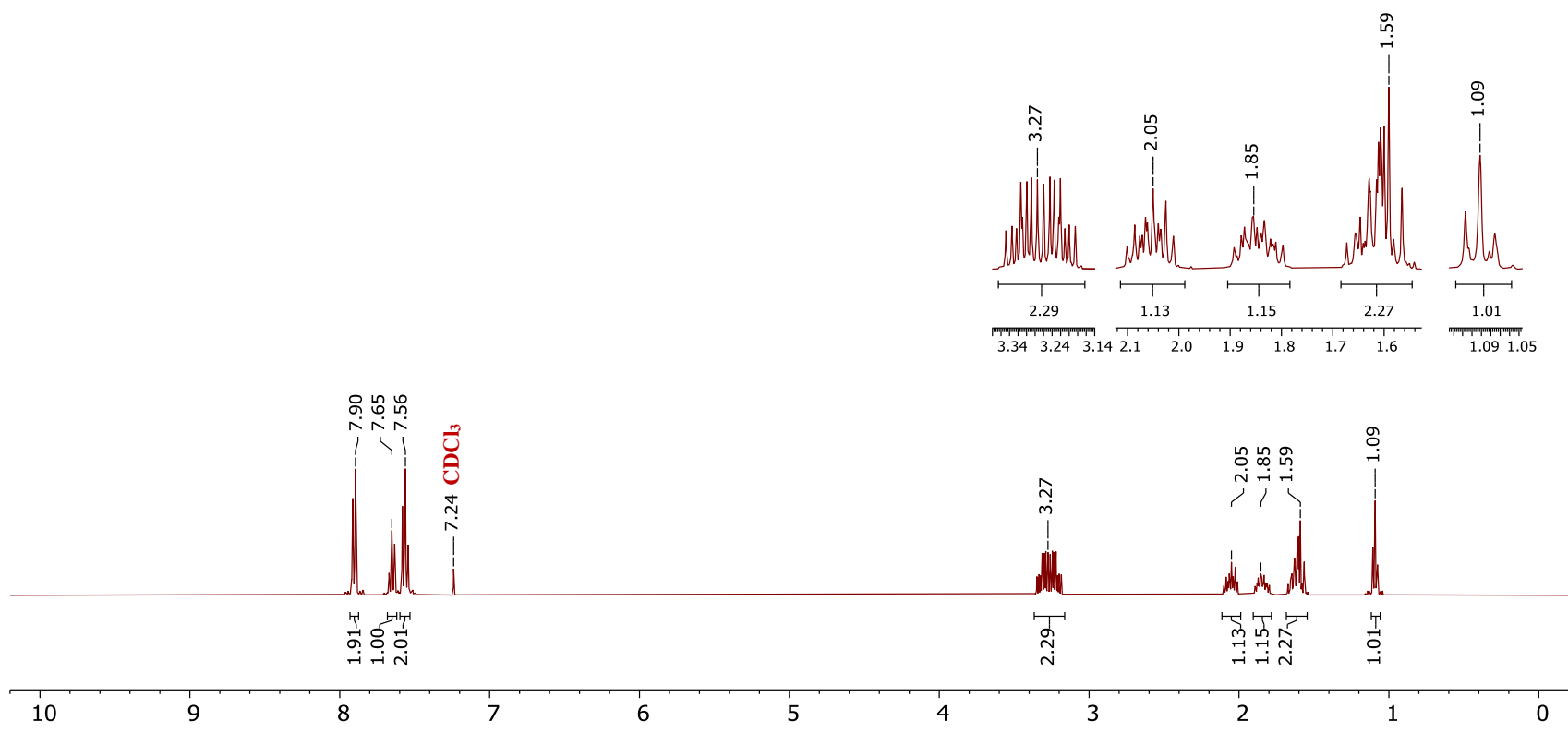

${ }^{13} \mathrm{C}$ NMR (100 $\left.\mathrm{MHz}, \mathrm{CDCl}_{3}\right)$

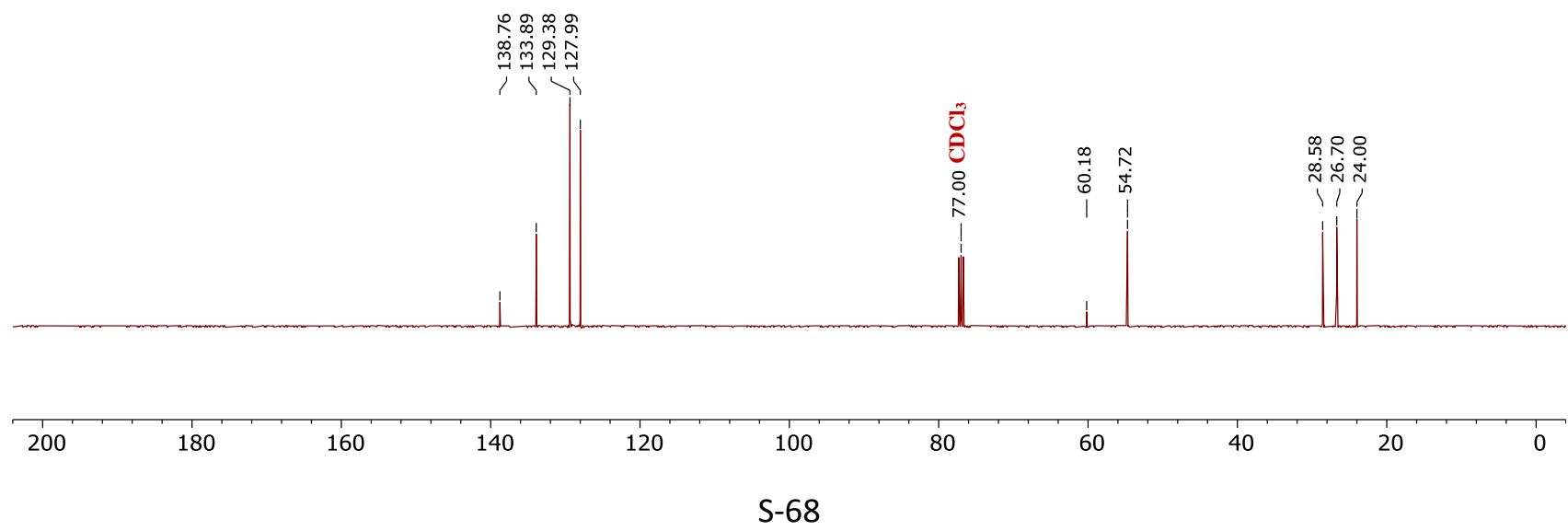


<smiles>CN1CCC(CS(=O)(=O)c2ccccc2)CC1</smiles>

$2 h$

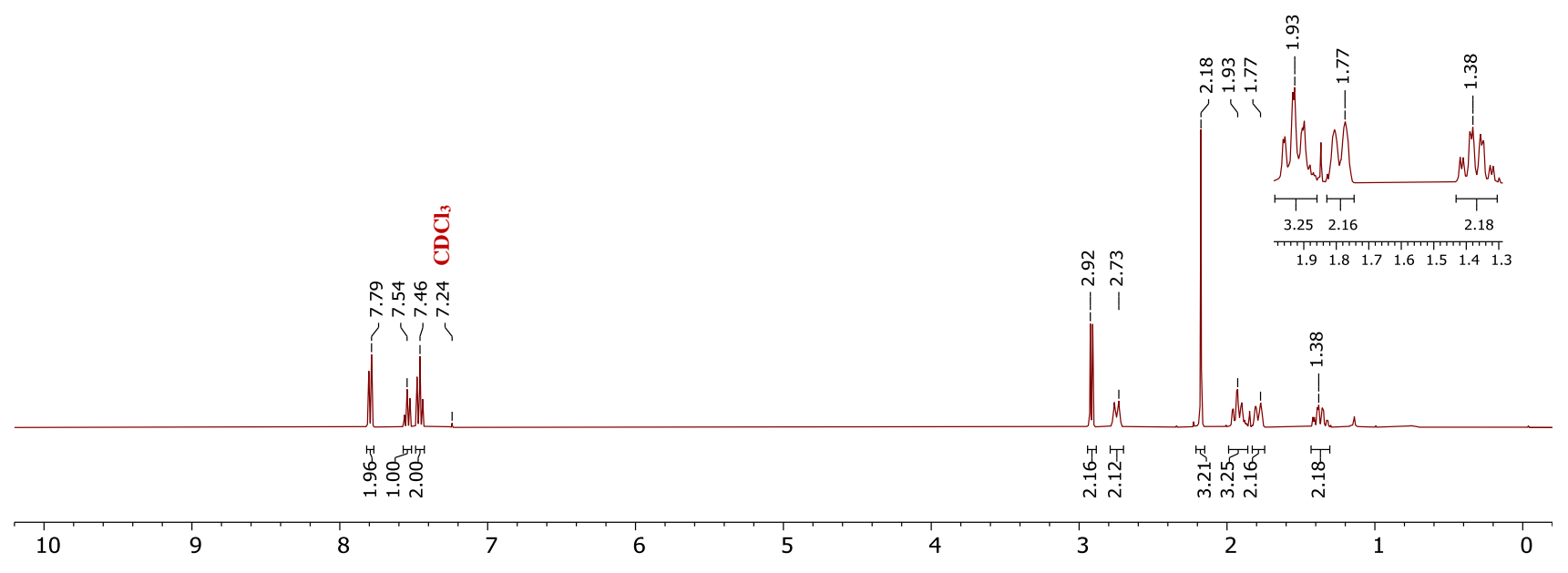

${ }^{13} \mathrm{C}$ NMR (100 MHz, $\left.\mathrm{CDCl}_{3}\right)$

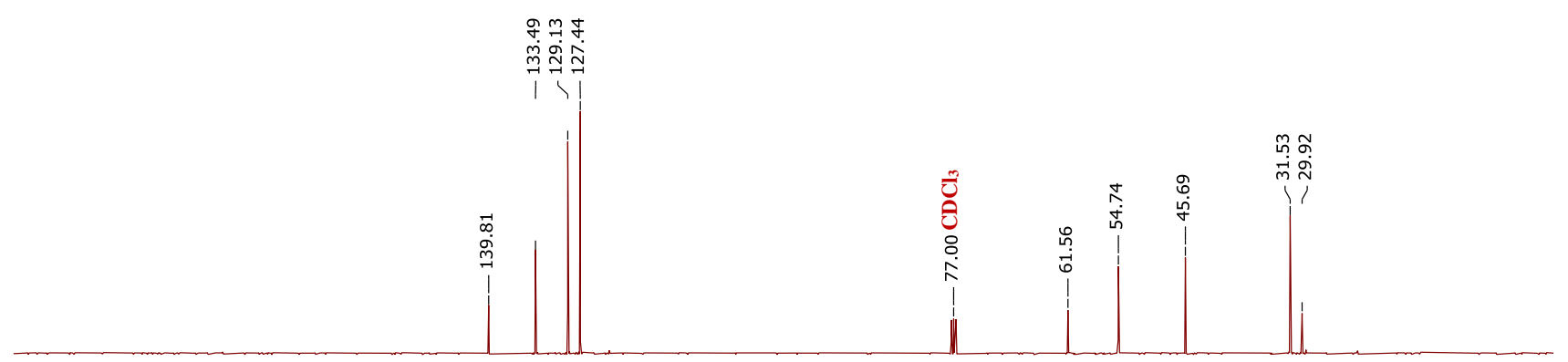




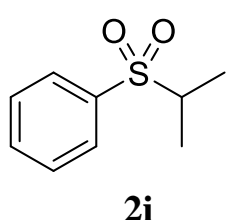

${ }^{1} \mathrm{H}$ NMR (400 MHz, $\mathrm{CDCl}_{3}$ )

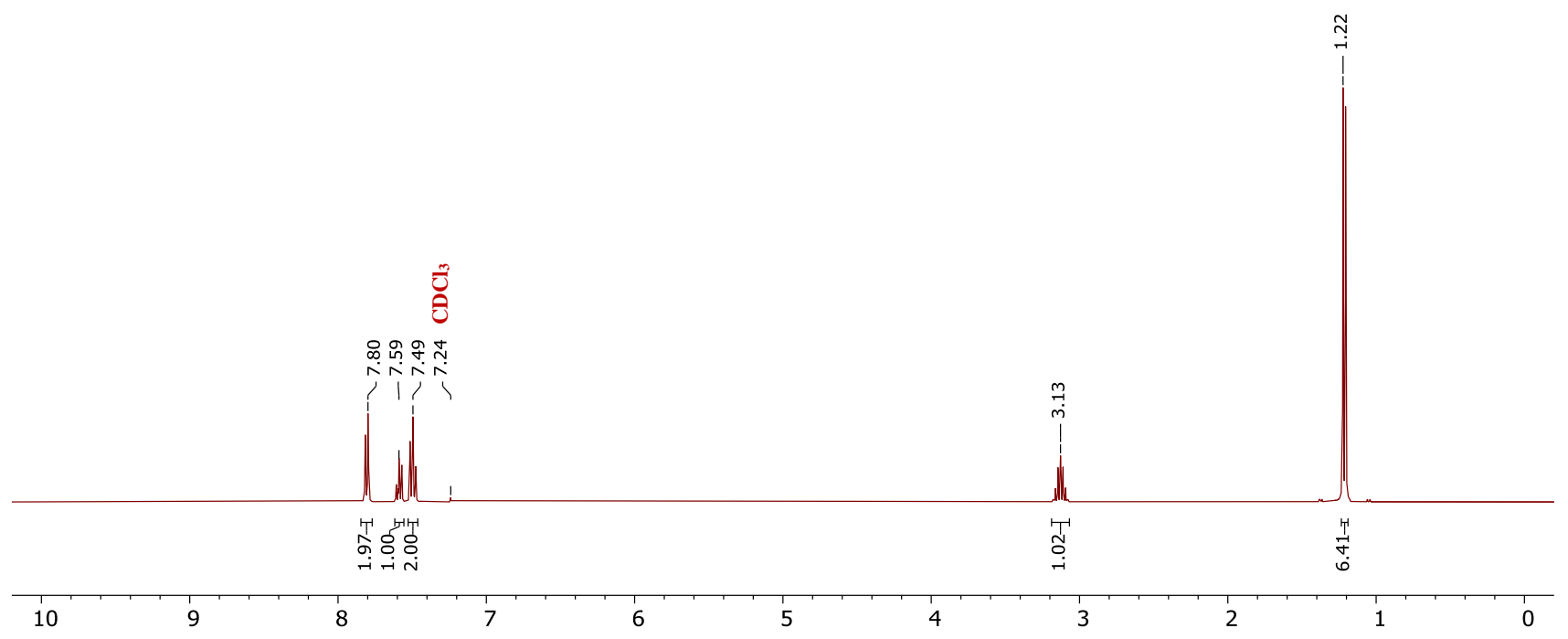

${ }^{13} \mathrm{C}$ NMR (100 MHz, $\left.\mathrm{CDCl}_{3}\right)$

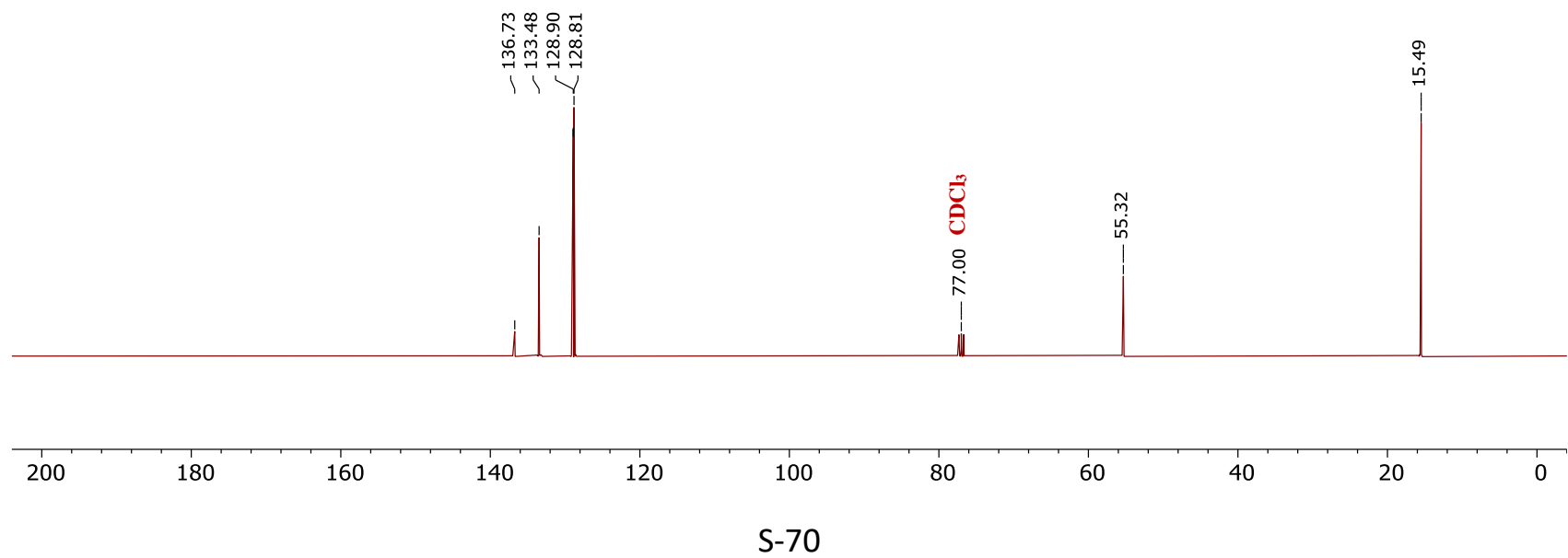




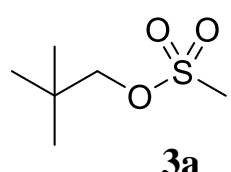

${ }^{1} \mathrm{H}$ NMR (400 MHz, $\left.\mathrm{CDCl}_{3}\right)$

3a

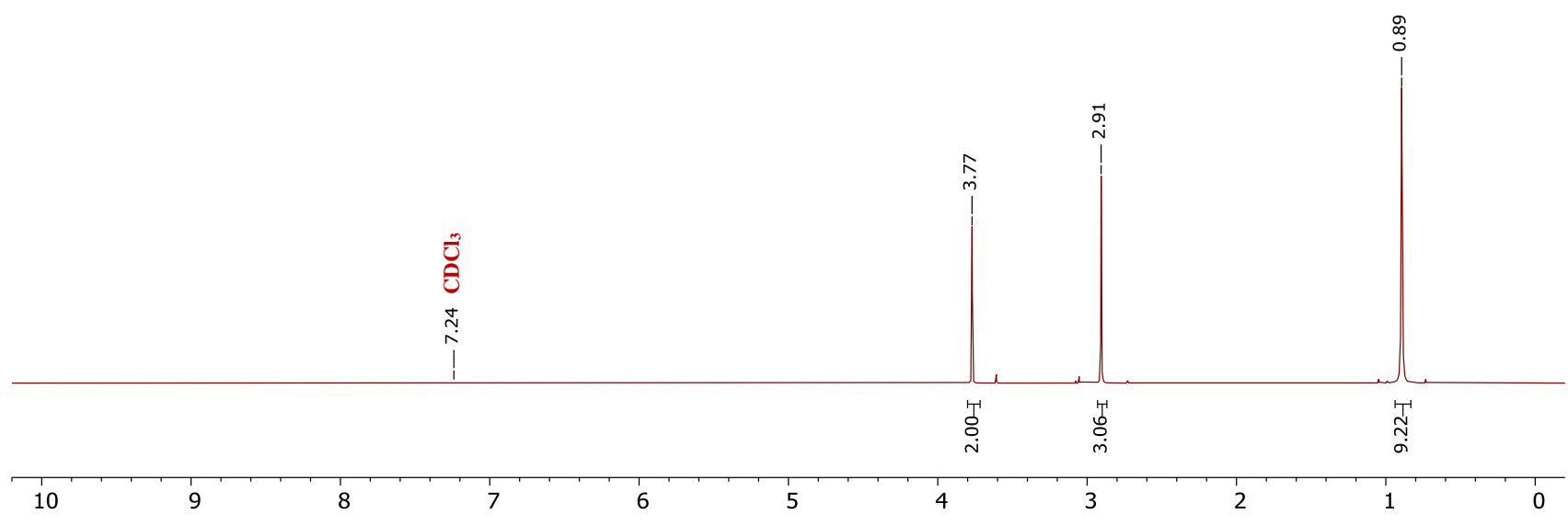

${ }^{13} \mathrm{C}$ NMR (100 MHz, $\left.\mathrm{CDCl}_{3}\right)$

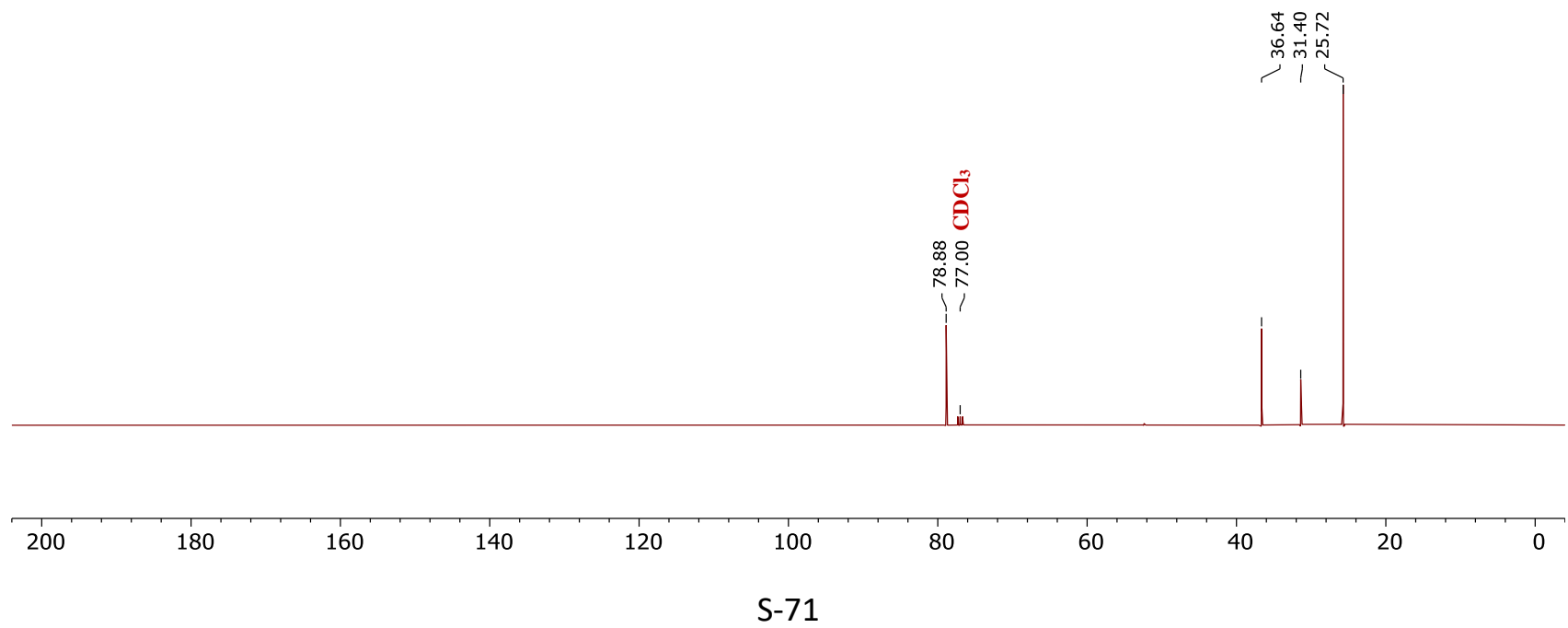




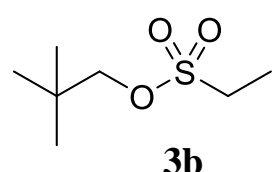

${ }^{1} \mathrm{H}$ NMR (400 MHz, $\left.\mathrm{CDCl}_{3}\right)$

3b

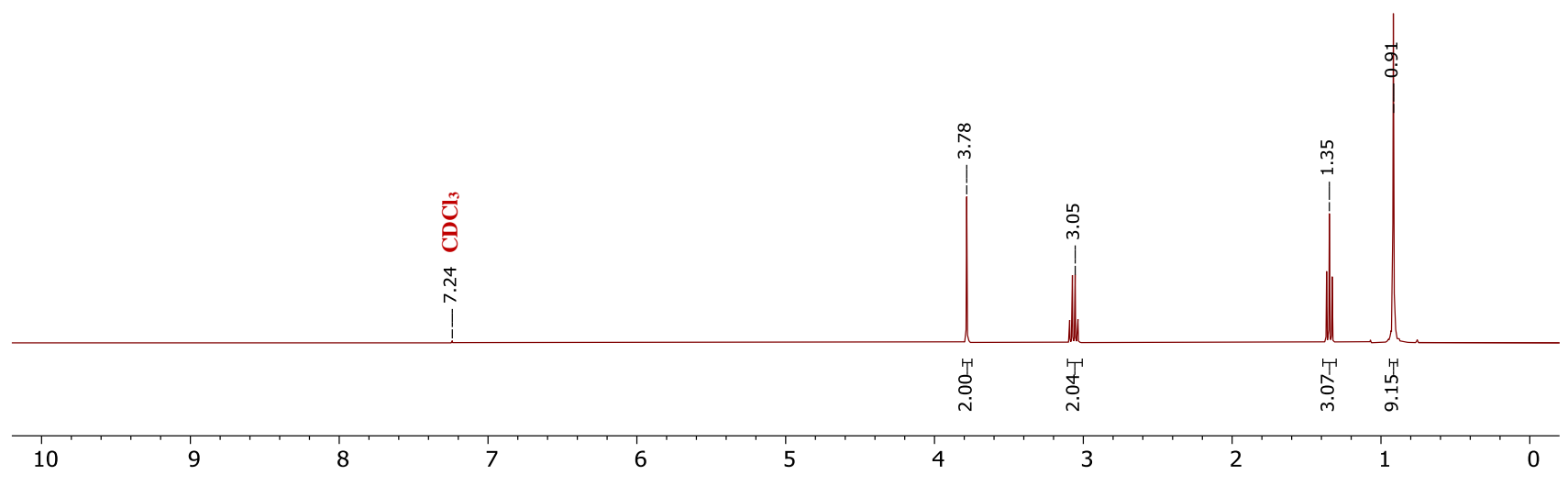

${ }^{13} \mathrm{C}$ NMR (100 MHz, $\left.\mathrm{CDCl}_{3}\right)$

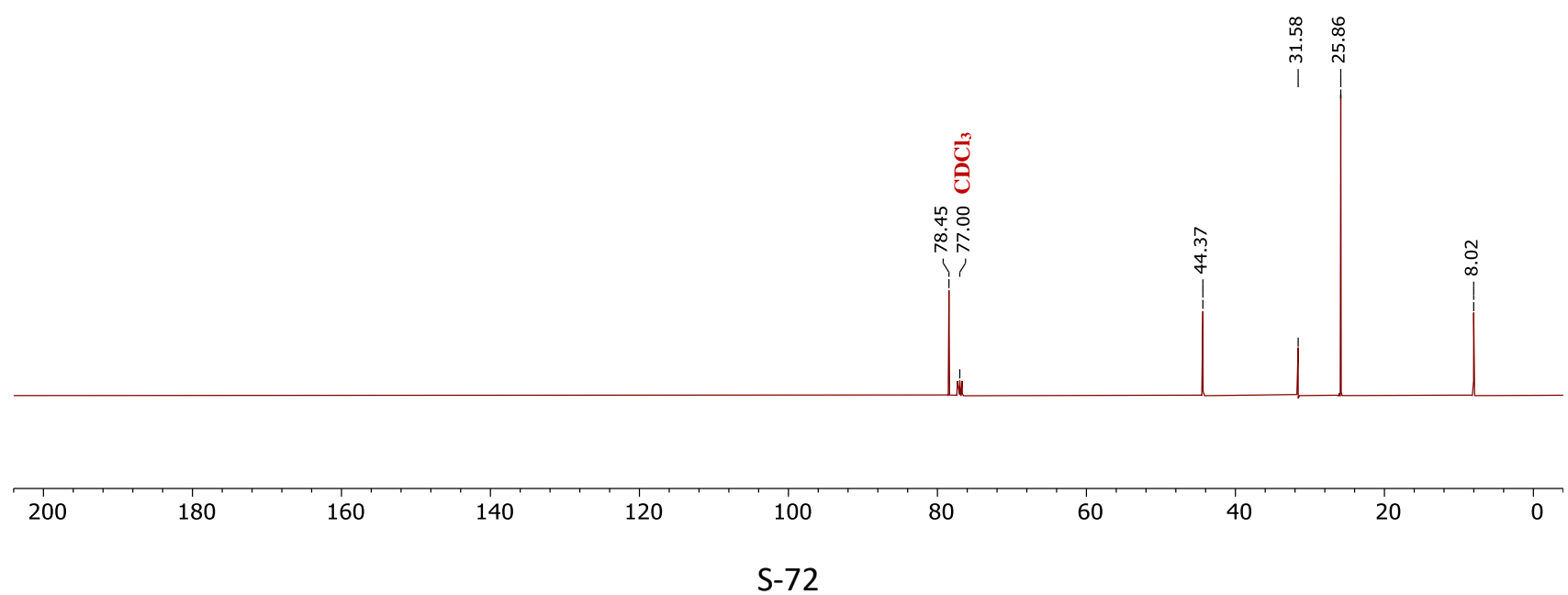




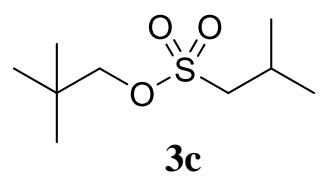

${ }^{1} \mathrm{H}$ NMR (400 MHz, $\left.\mathrm{CDCl}_{3}\right)$

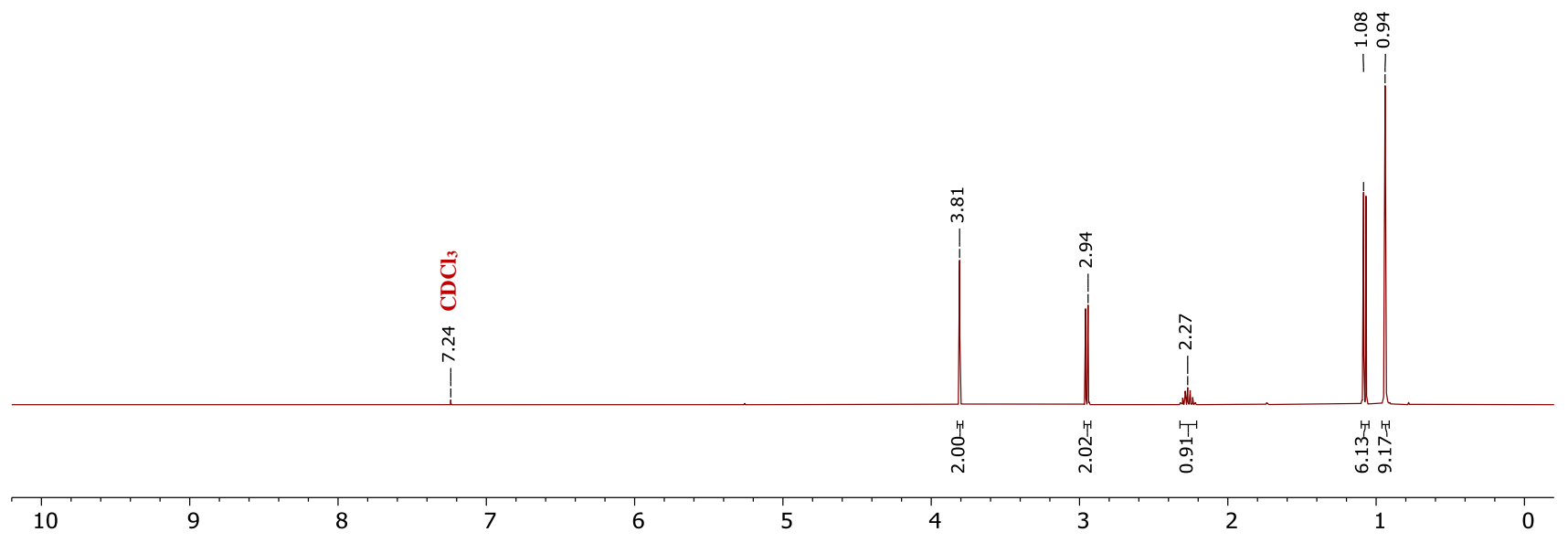

${ }^{13} \mathrm{C}$ NMR (100 MHz, $\left.\mathrm{CDCl}_{3}\right)$

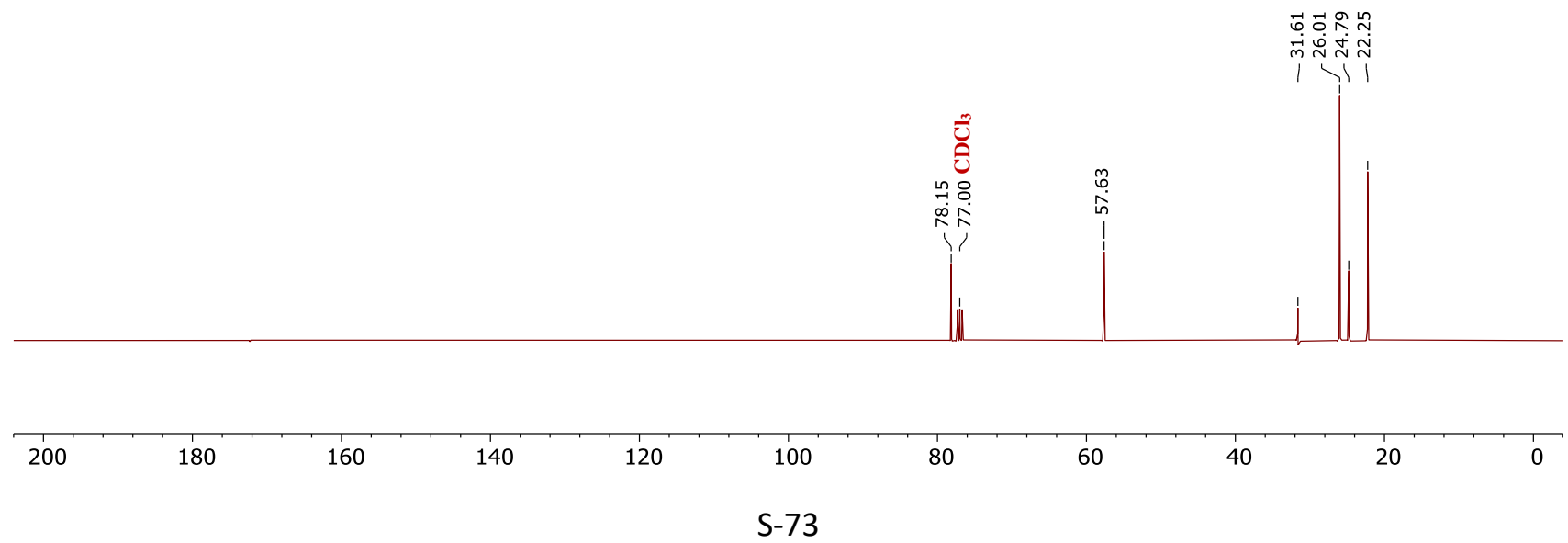




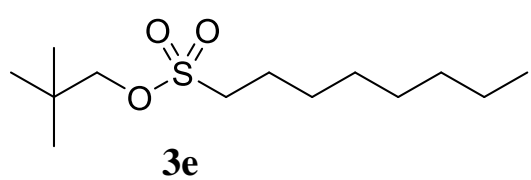

${ }^{1} \mathrm{H}$ NMR (400 MHz, $\left.\mathrm{CDCl}_{3}\right)$

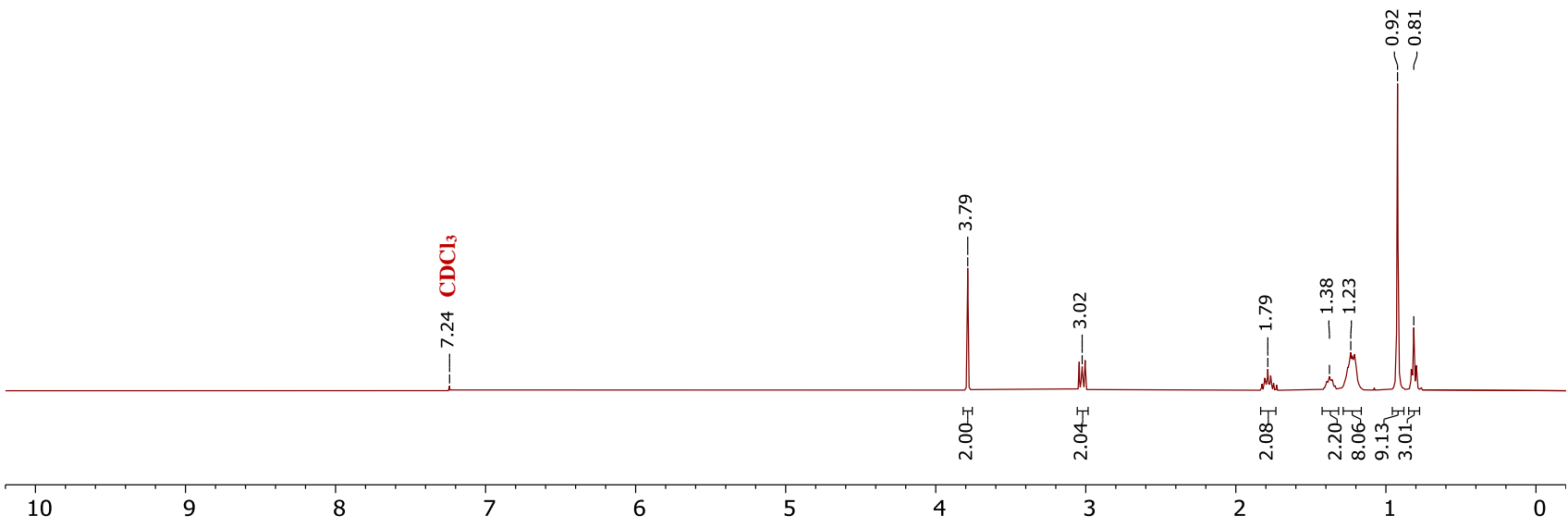

${ }^{13} \mathrm{C}$ NMR (100 MHz, $\left.\mathrm{CDCl}_{3}\right)$

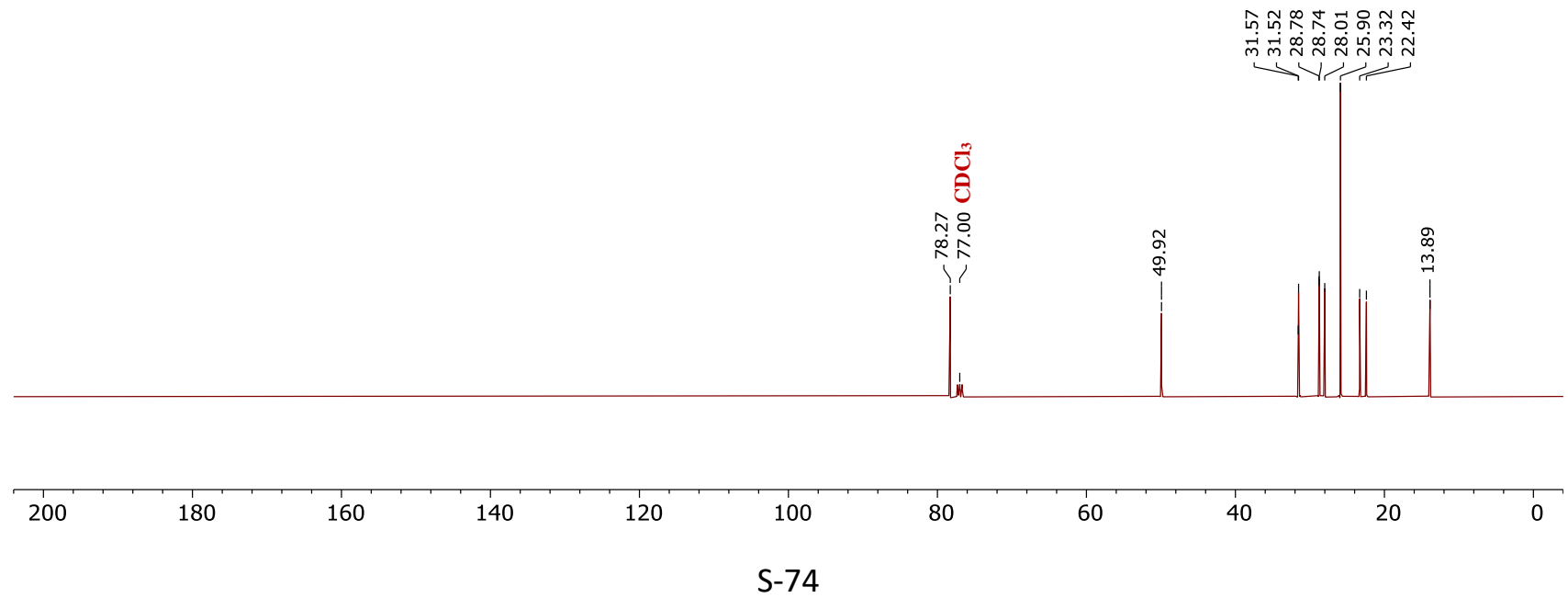


<smiles>O=[N+]([O-])c1ccc([Sb]c2ccccc2)nc1</smiles>
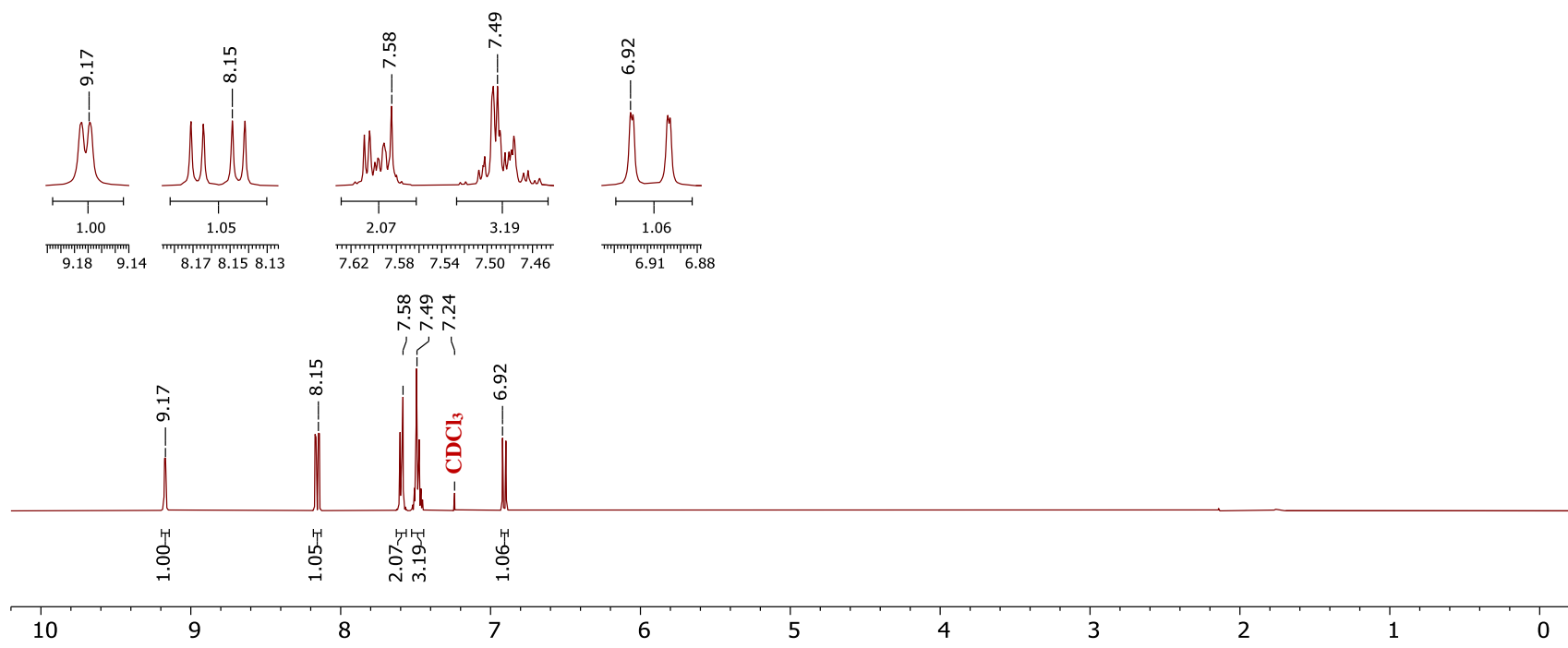

${ }^{13} \mathrm{C}$ NMR (100 MHz, $\left.\mathrm{CDCl}_{3}\right)$

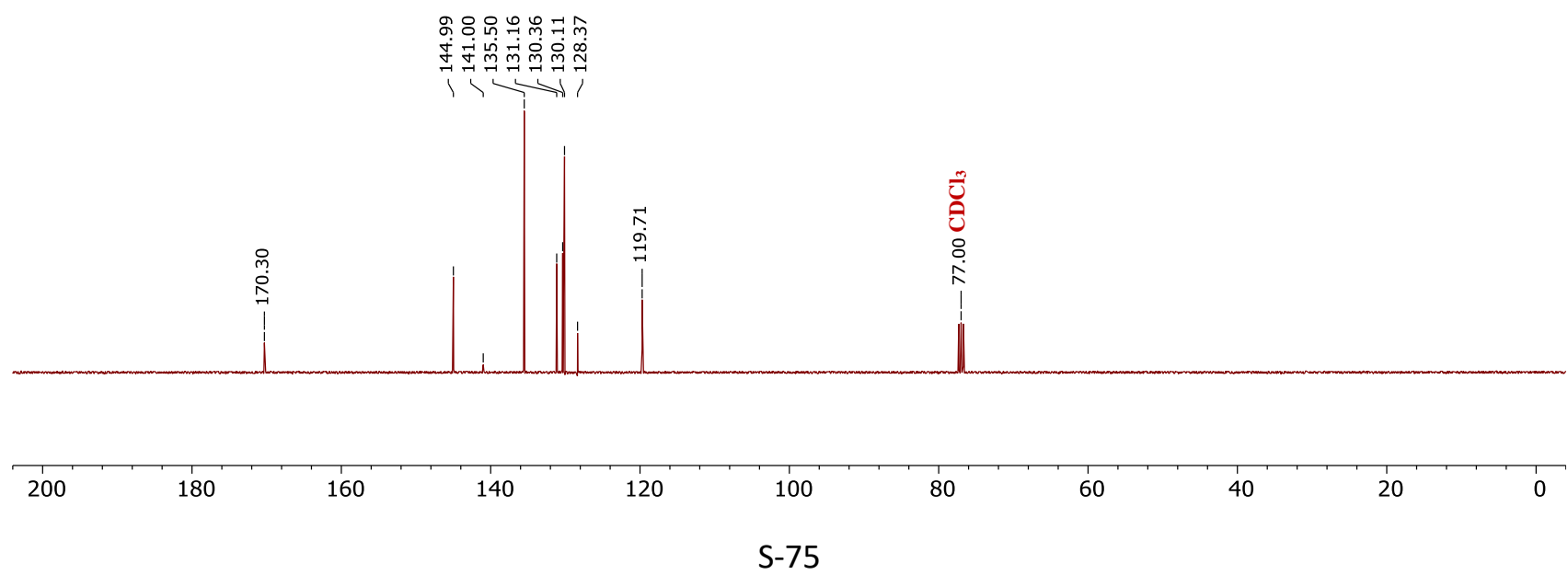




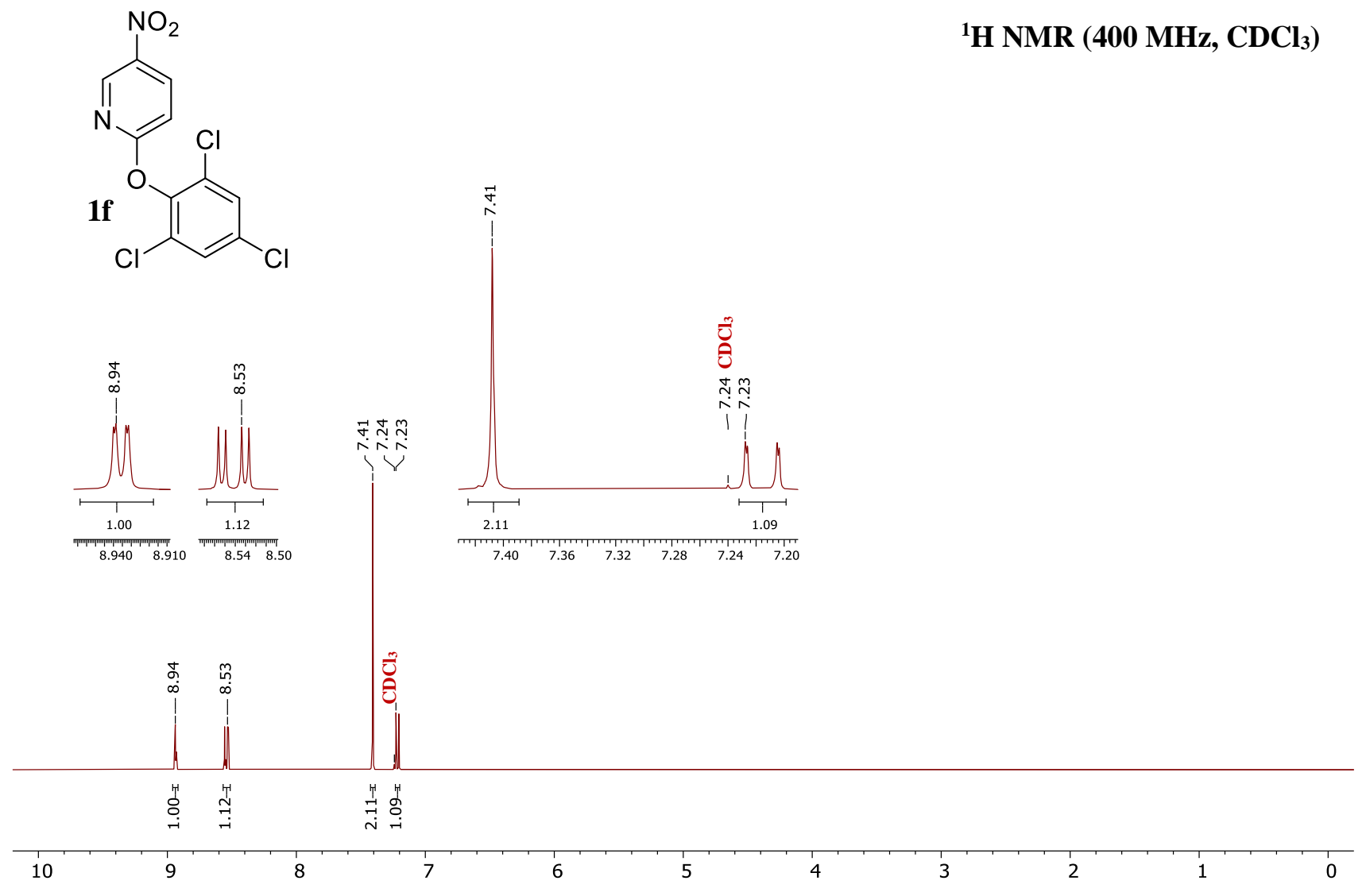

${ }^{13} \mathrm{C}$ NMR (100 MHz, $\left.\mathrm{CDCl}_{3}\right)$

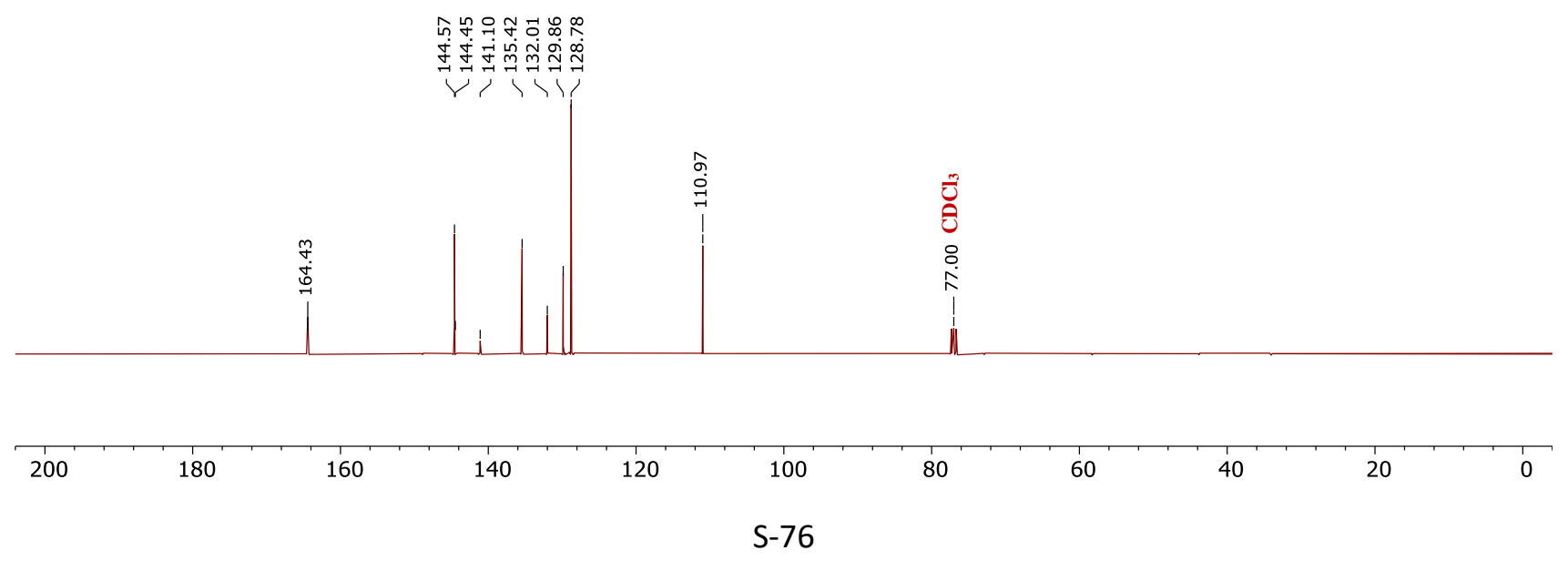


<smiles>O=[N+]([O-])c1cnc2ccccc2c1</smiles>
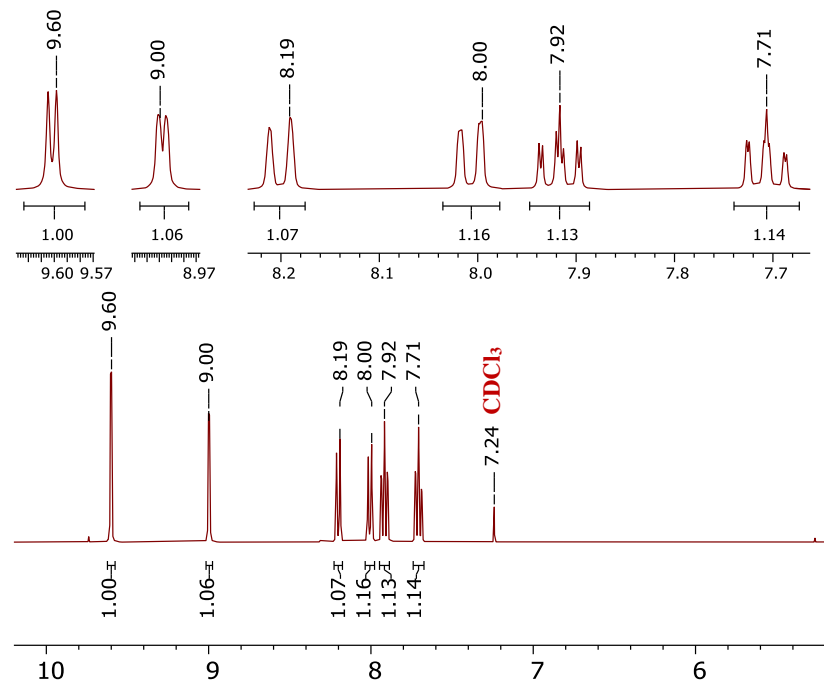

6

3

${ }^{13} \mathrm{C}$ NMR (100 MHz, $\left.\mathrm{CDCl}_{3}\right)$

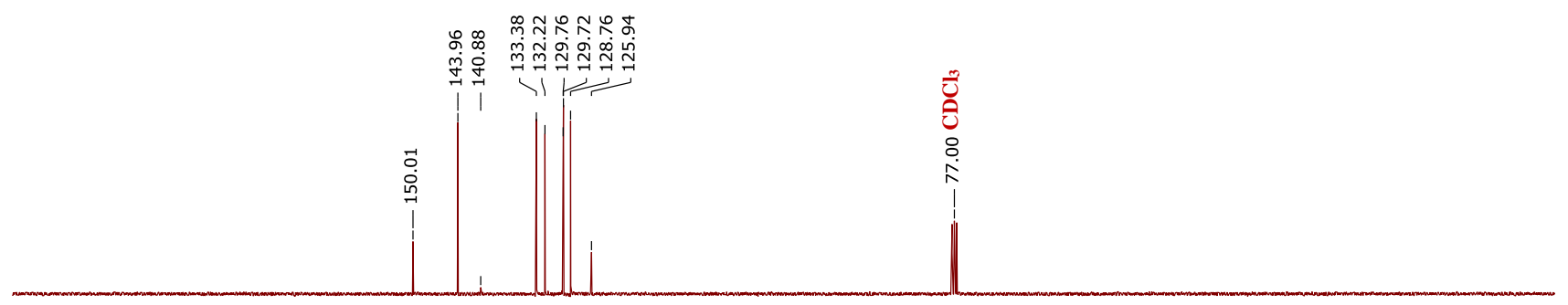


<smiles>Cc1ccncc1[N+](=O)[O-]</smiles>

$4 a$

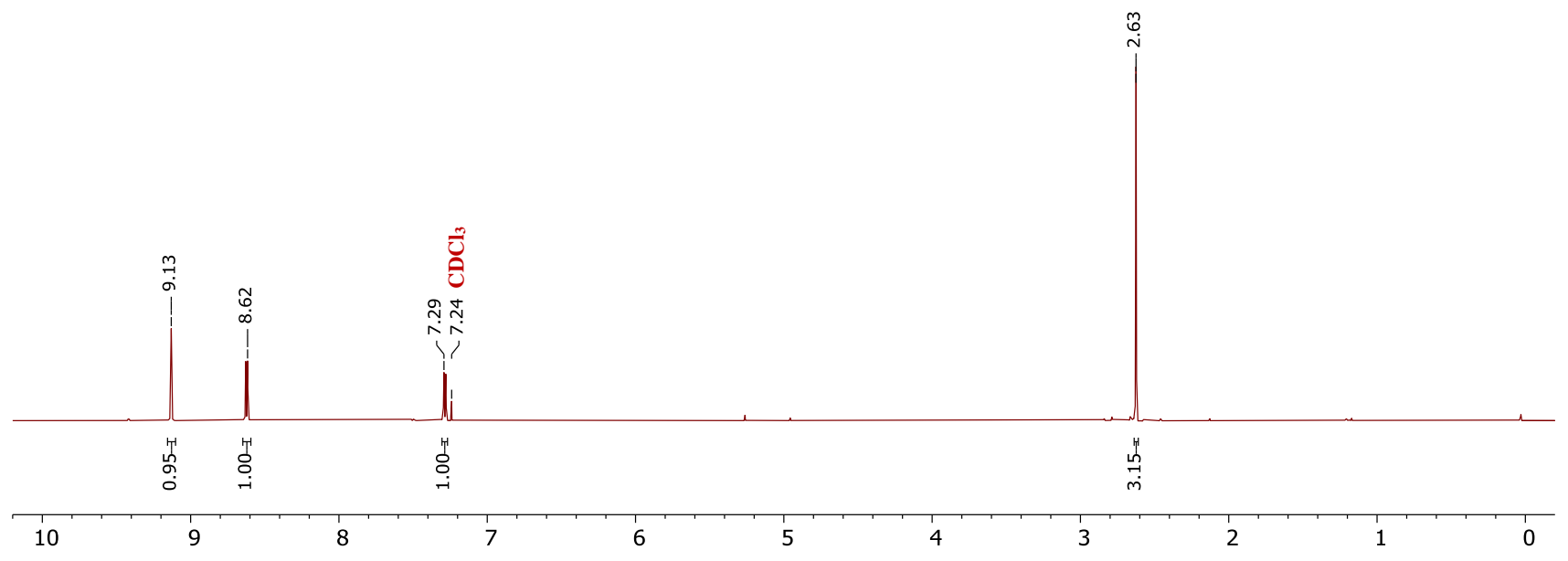

${ }^{13} \mathrm{C}$ NMR (100 MHz, $\left.\mathrm{CDCl}_{3}\right)$

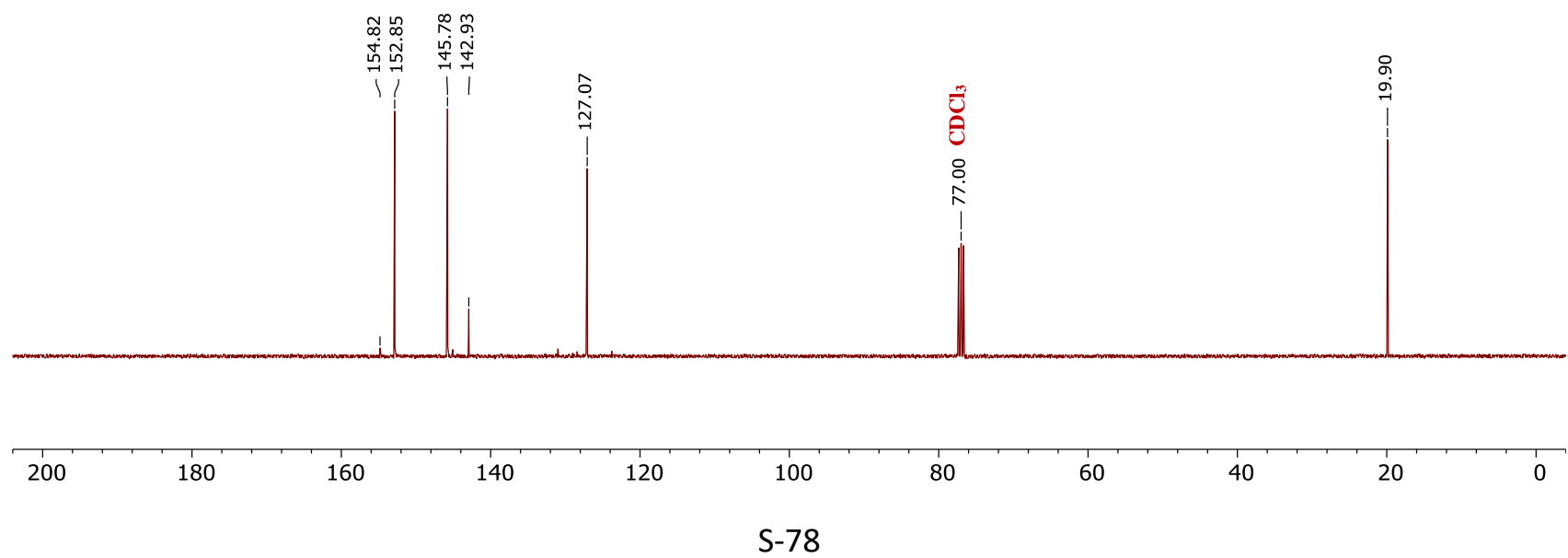


<smiles>CCc1ccncc1[N+](=O)[O-]</smiles>

4b

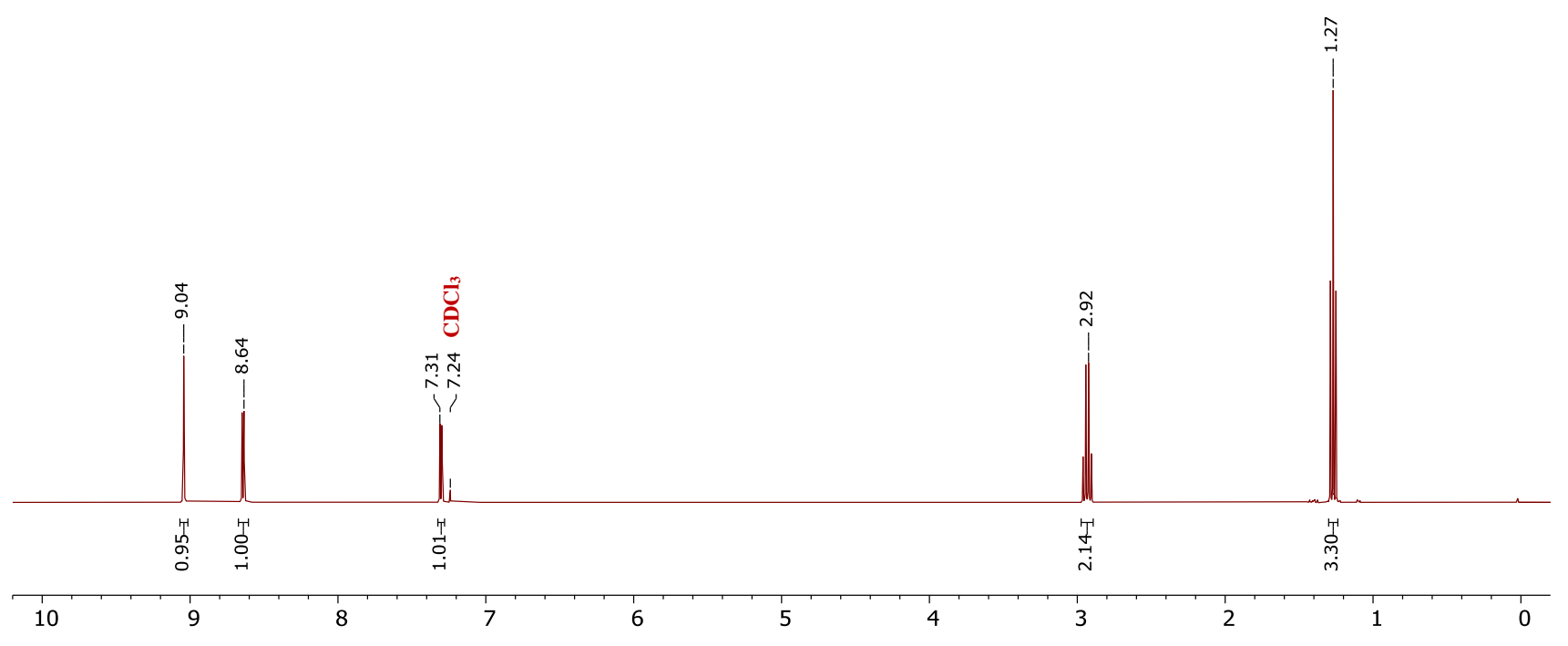

${ }^{13} \mathrm{C}$ NMR (100 MHz, $\left.\mathrm{CDCl}_{3}\right)$

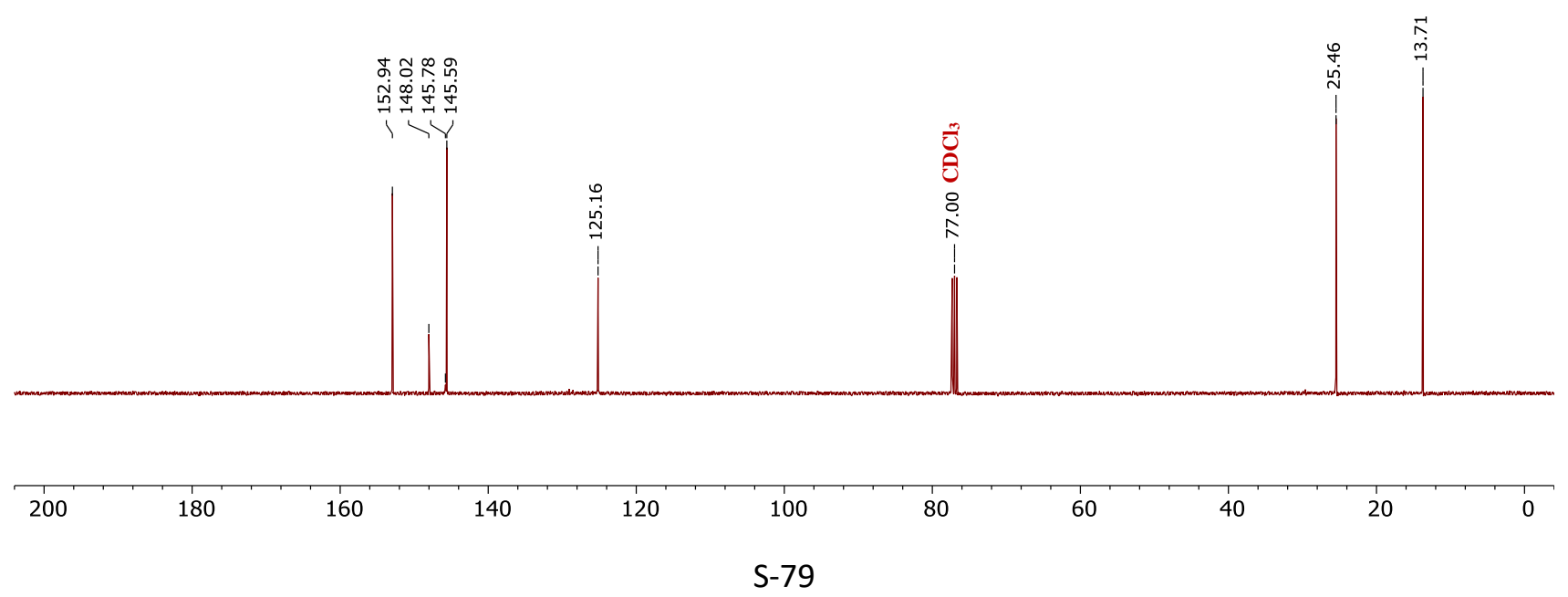


<smiles>CC(C)Cc1ccncc1[N+](=O)[O-]</smiles>

$4 c$

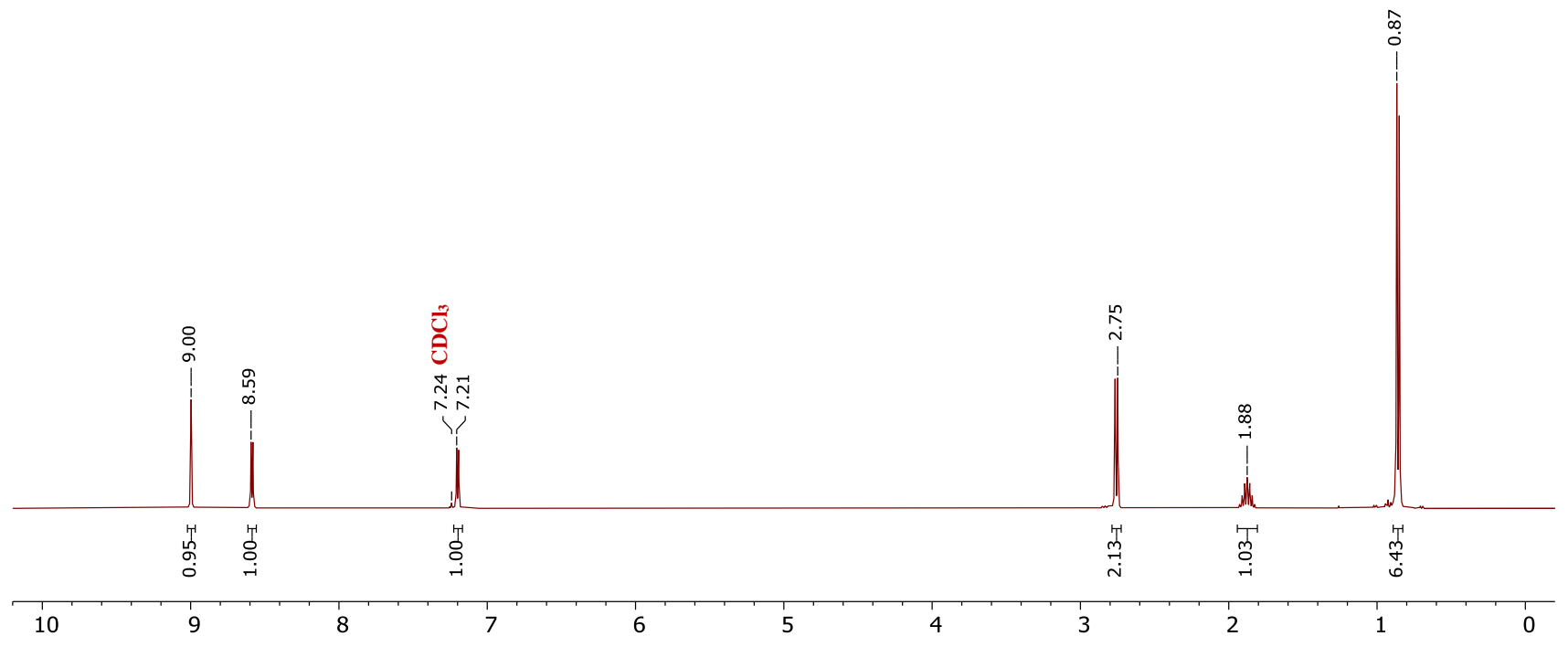

${ }^{13} \mathrm{C}$ NMR (100 MHz, $\left.\mathrm{CDCl}_{3}\right)$

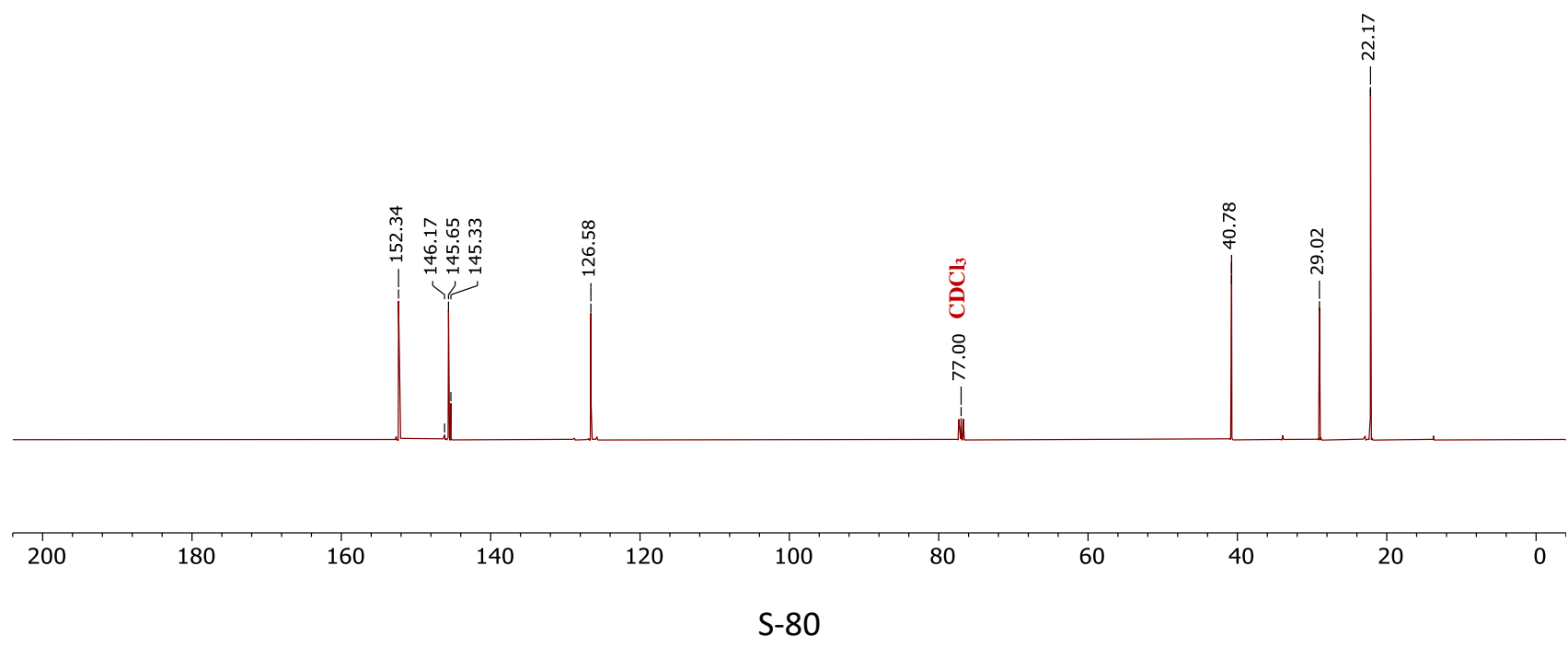


<smiles>CC(C)Cc1ccc([N+](=O)[O-])cn1</smiles>

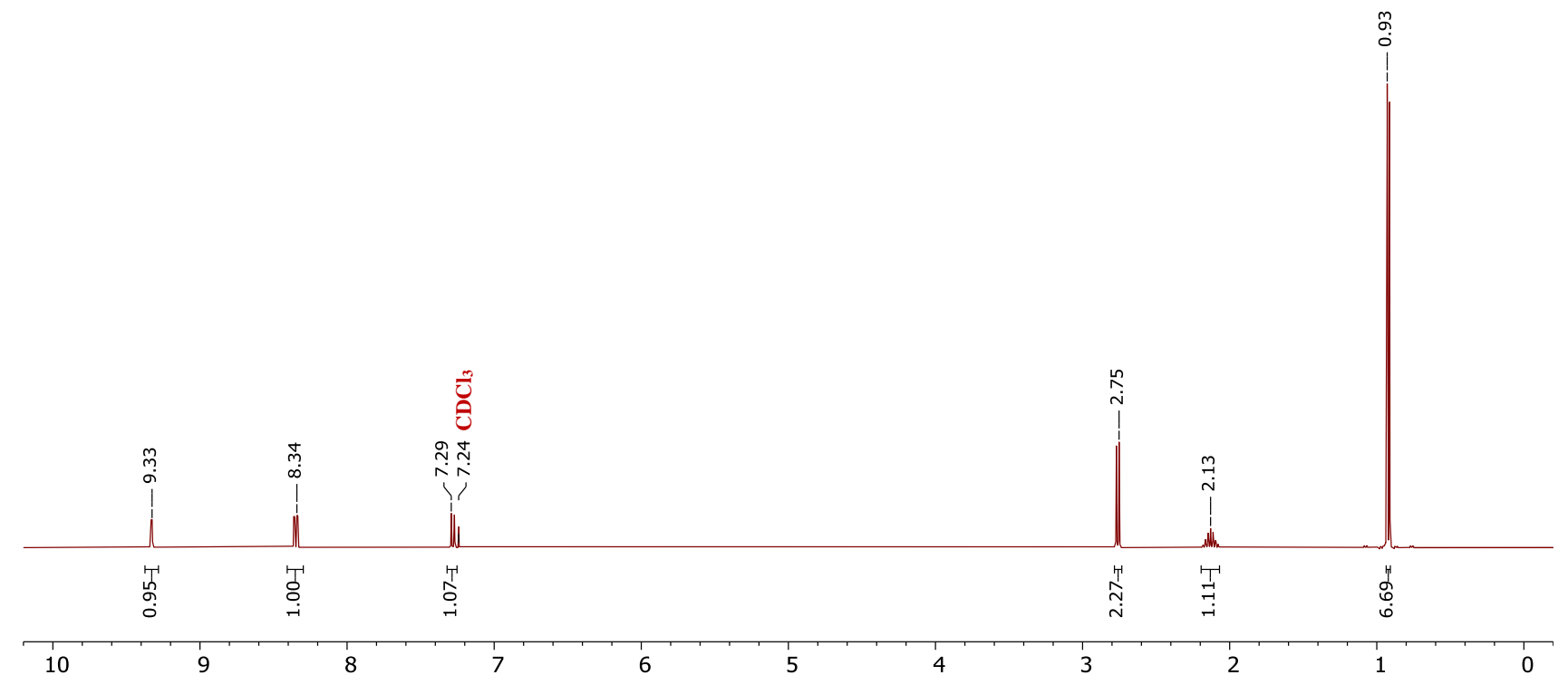

${ }^{13} \mathrm{C}$ NMR (100 MHz, $\left.\mathrm{CDCl}_{3}\right)$

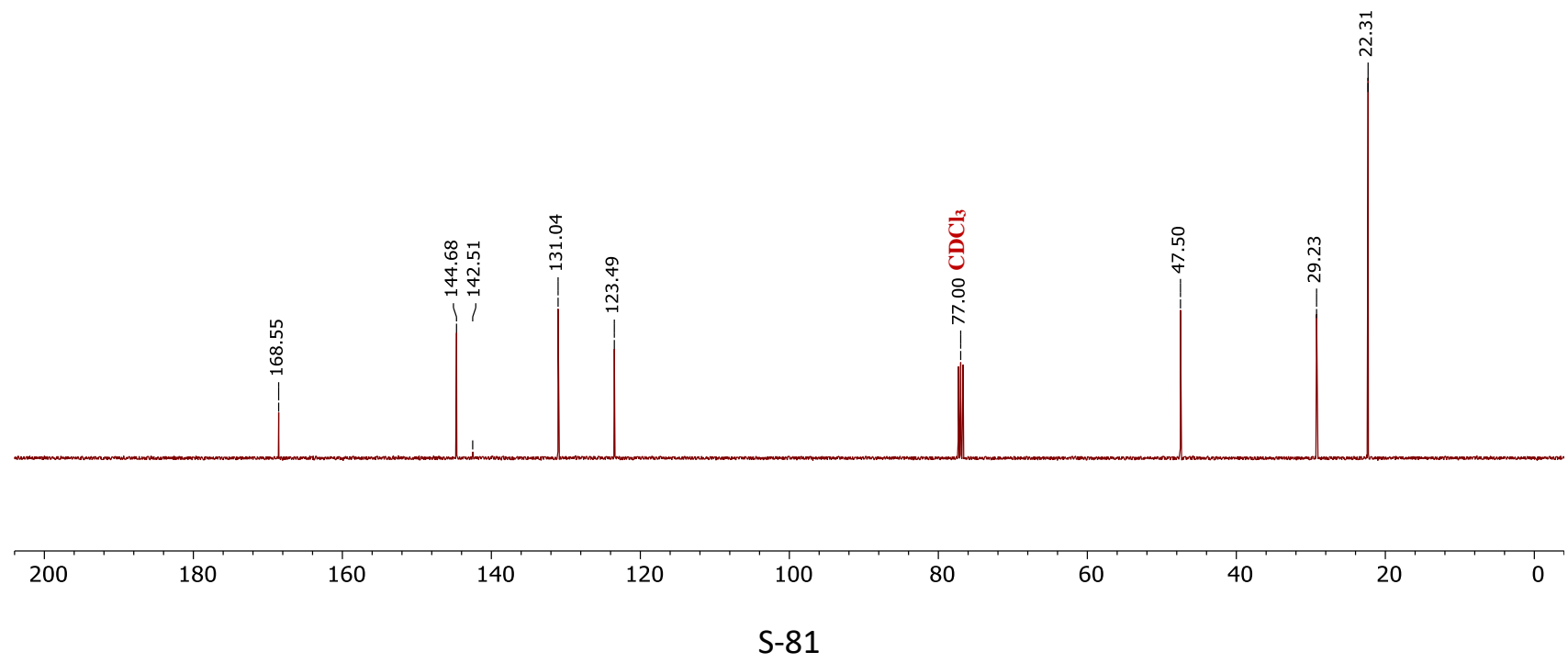


<smiles>CC(C)(C)Cc1ccncc1[N+](=O)[O-]</smiles>

4d

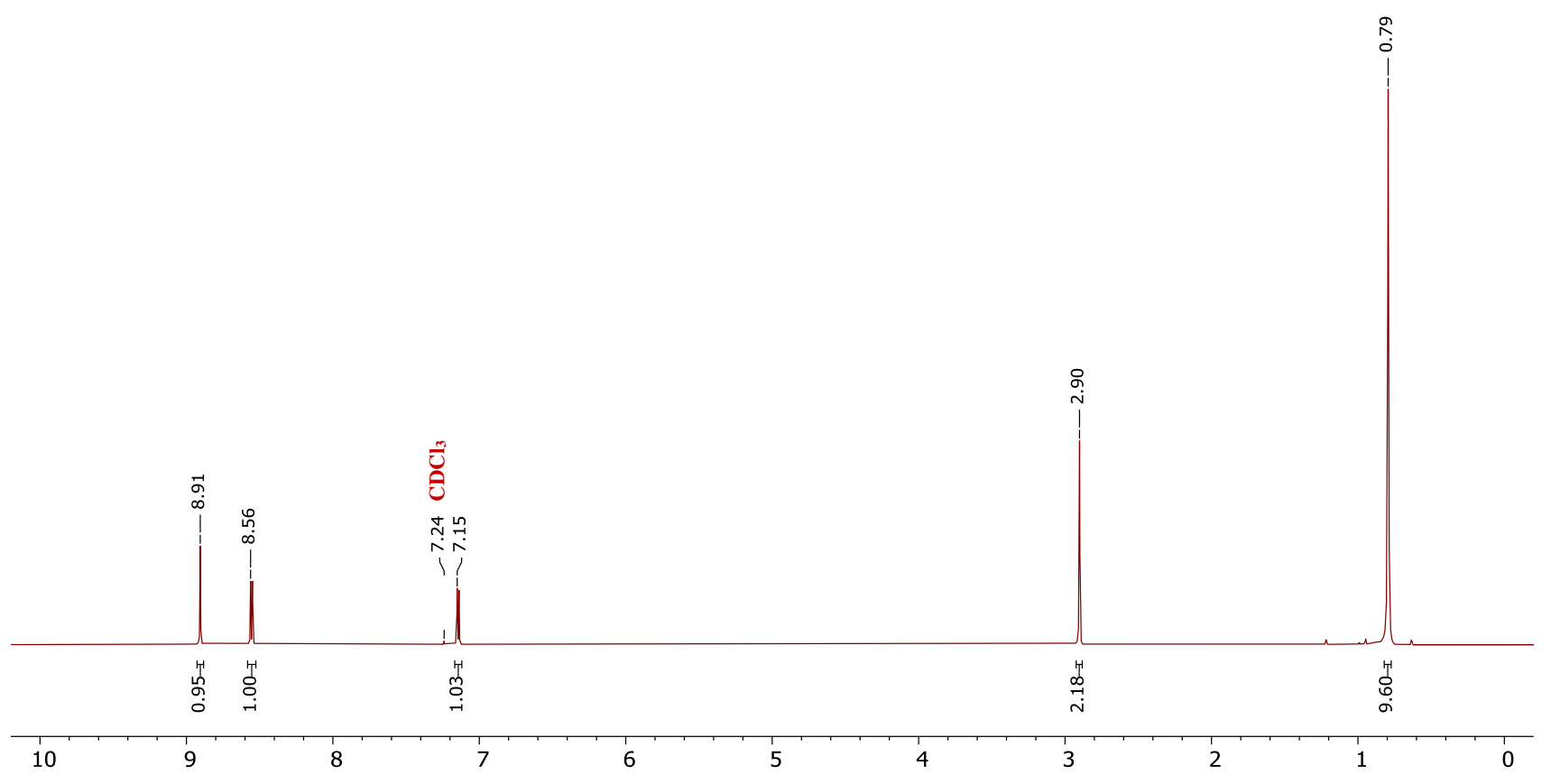

${ }^{13} \mathrm{C}$ NMR (100 MHz, $\left.\mathrm{CDCl}_{3}\right)$

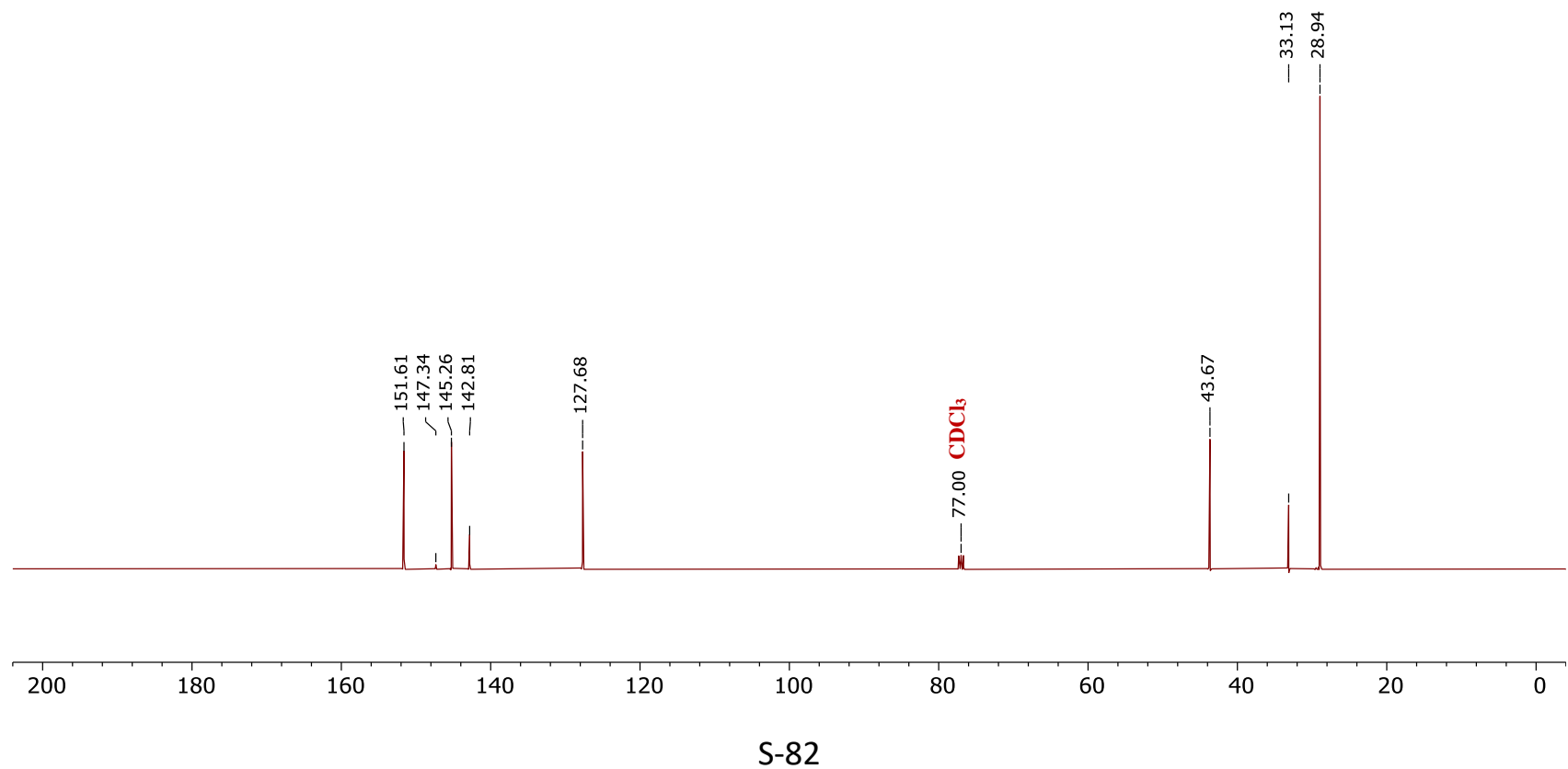


<smiles>CCCCCCCc1ccncc1[N+](=O)[O-]</smiles>

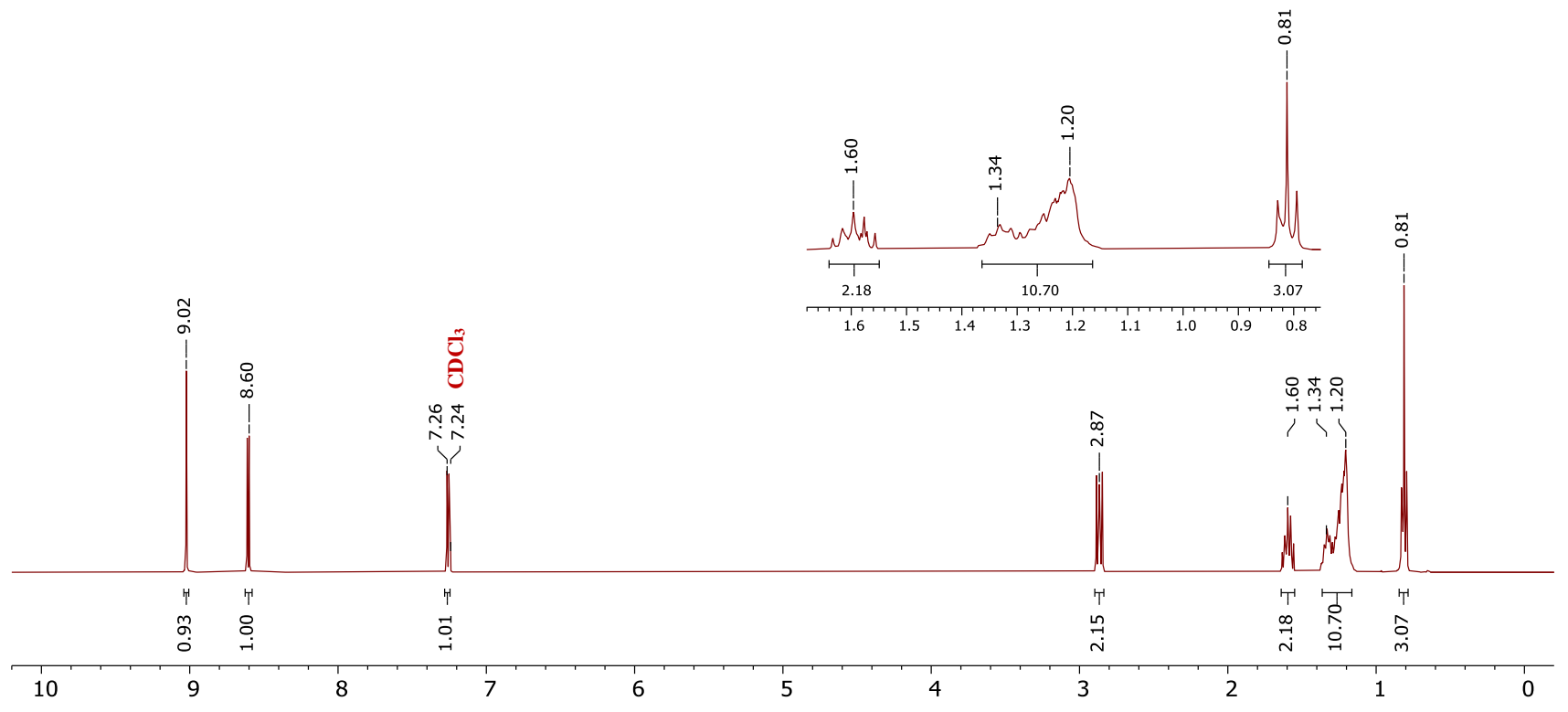

${ }^{13} \mathrm{C}$ NMR (100 MHz, $\left.\mathrm{CDCl}_{3}\right)$

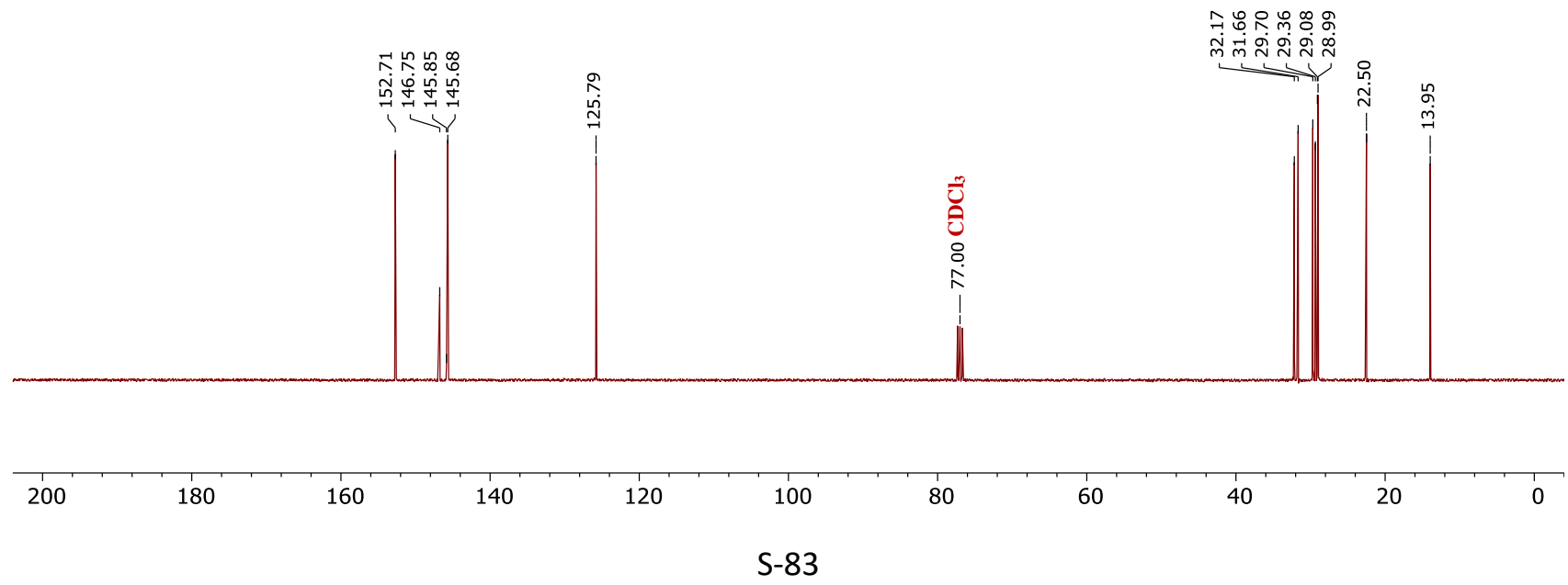


<smiles>CCCCCCCCc1ccc([N+](=O)[O-])cn1</smiles>

${ }^{1} \mathrm{H}$ NMR (400 MHz, $\left.\mathrm{CDCl}_{3}\right)$

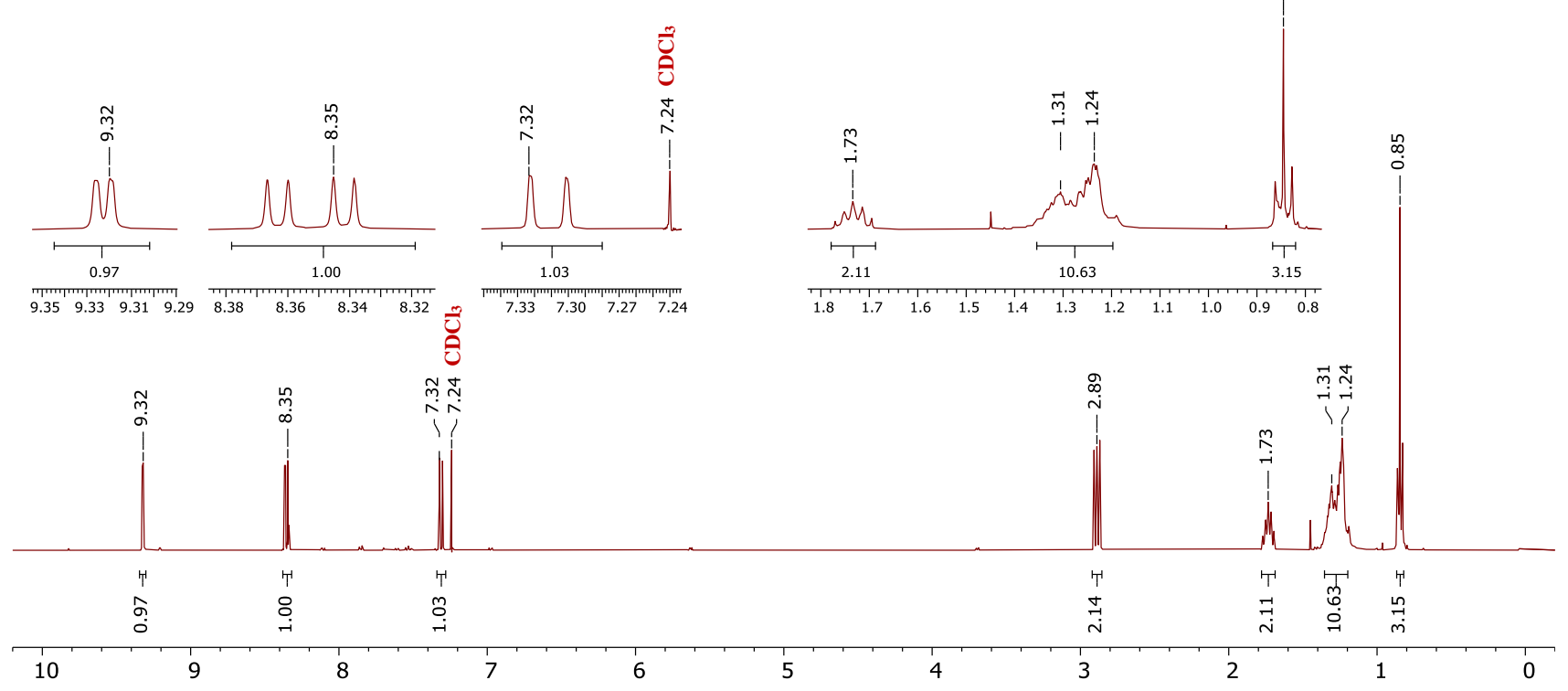

${ }^{13} \mathrm{C}$ NMR (100 MHz, $\left.\mathrm{CDCl}_{3}\right)$

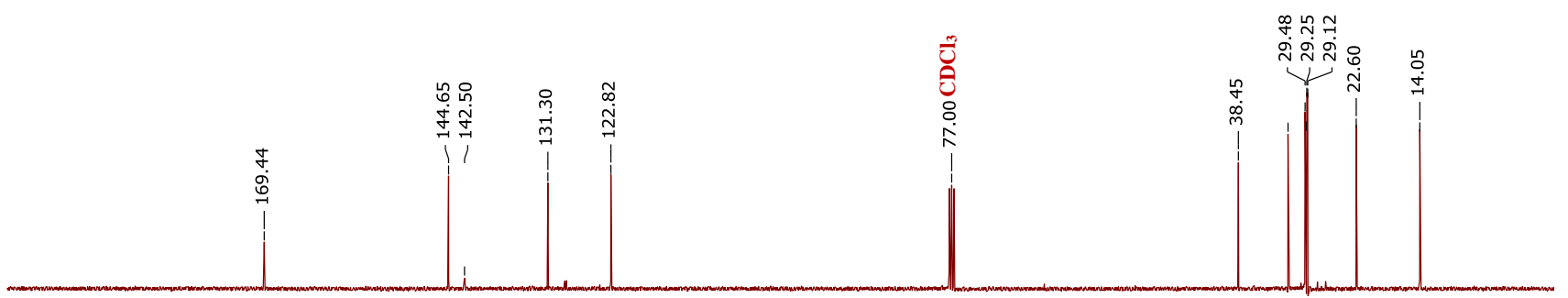


(N)

4f
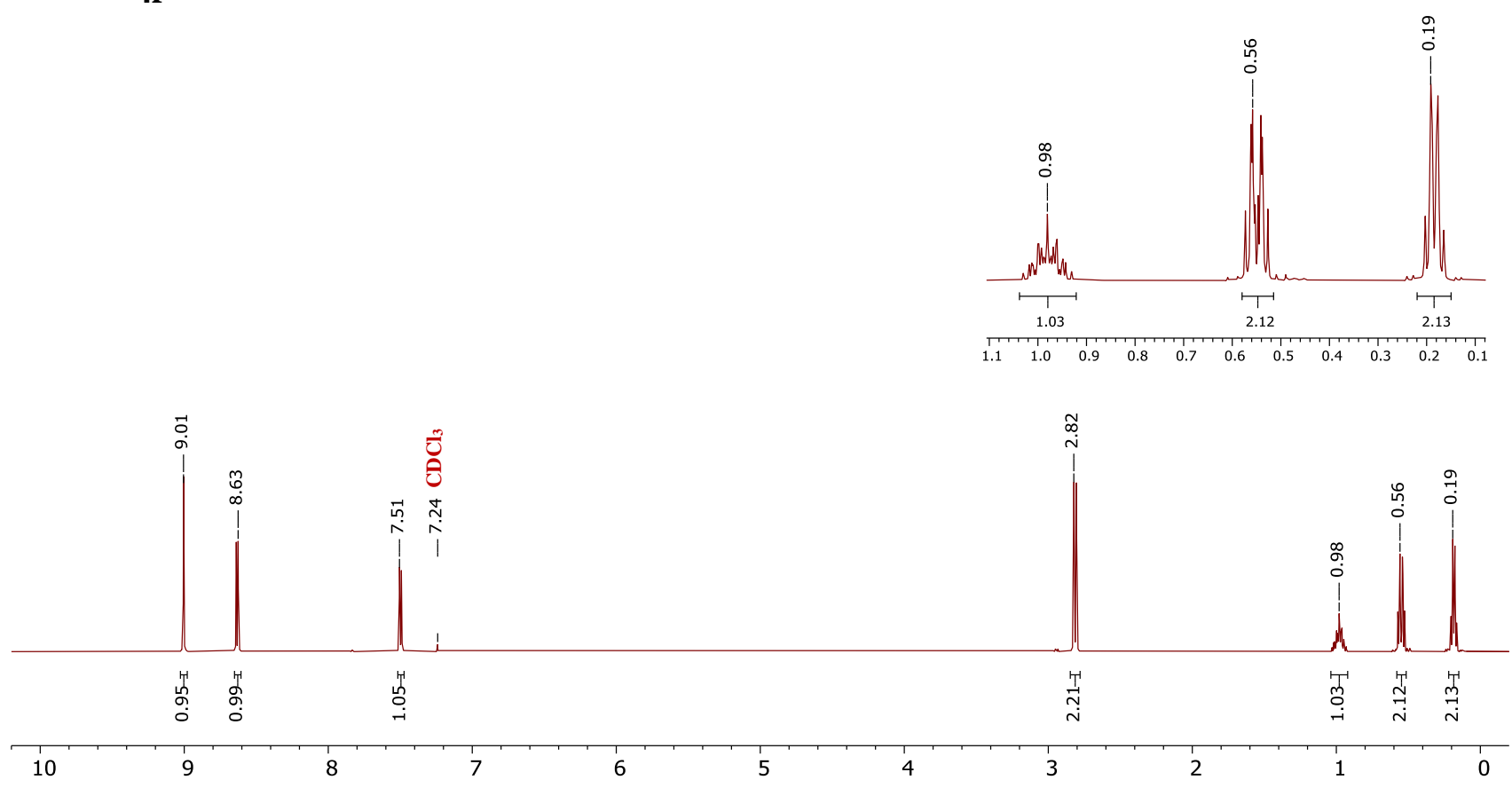

${ }^{13} \mathrm{C}$ NMR (100 MHz, $\left.\mathrm{CDCl}_{3}\right)$

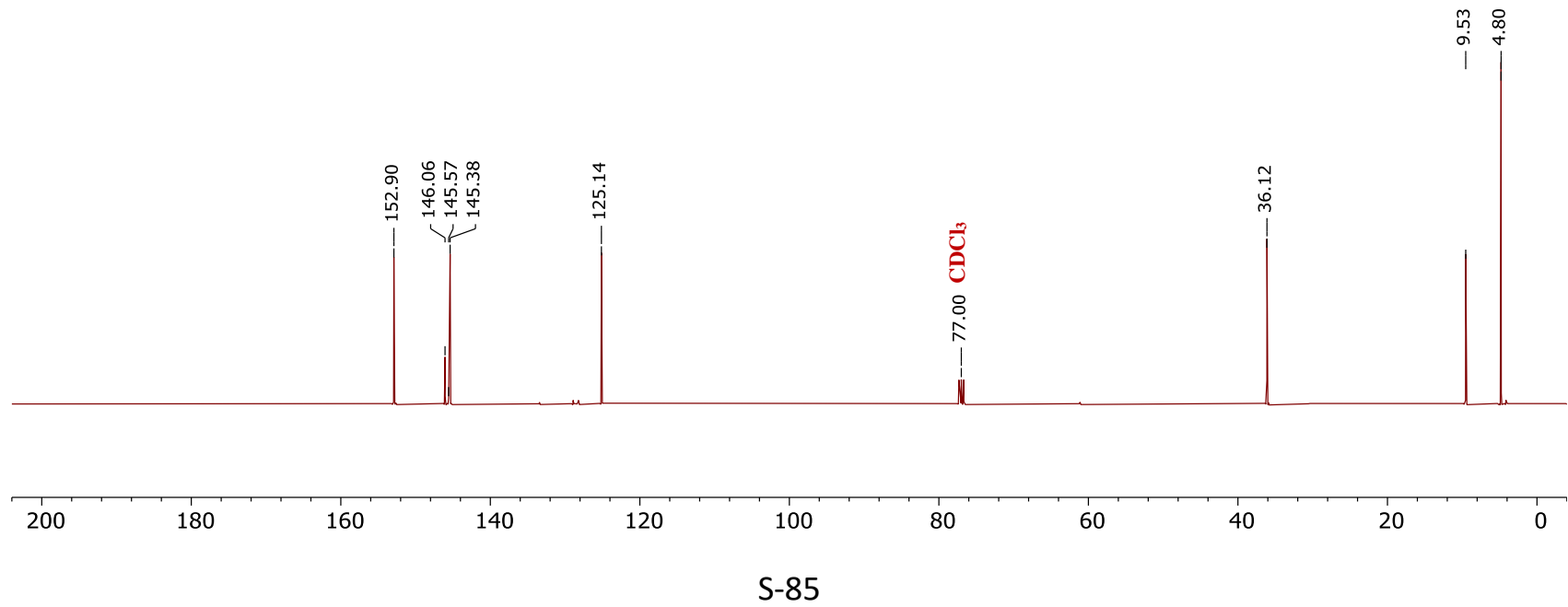


<smiles>O=[N+]([O-])c1ccc(CC2CC2)nc1</smiles>

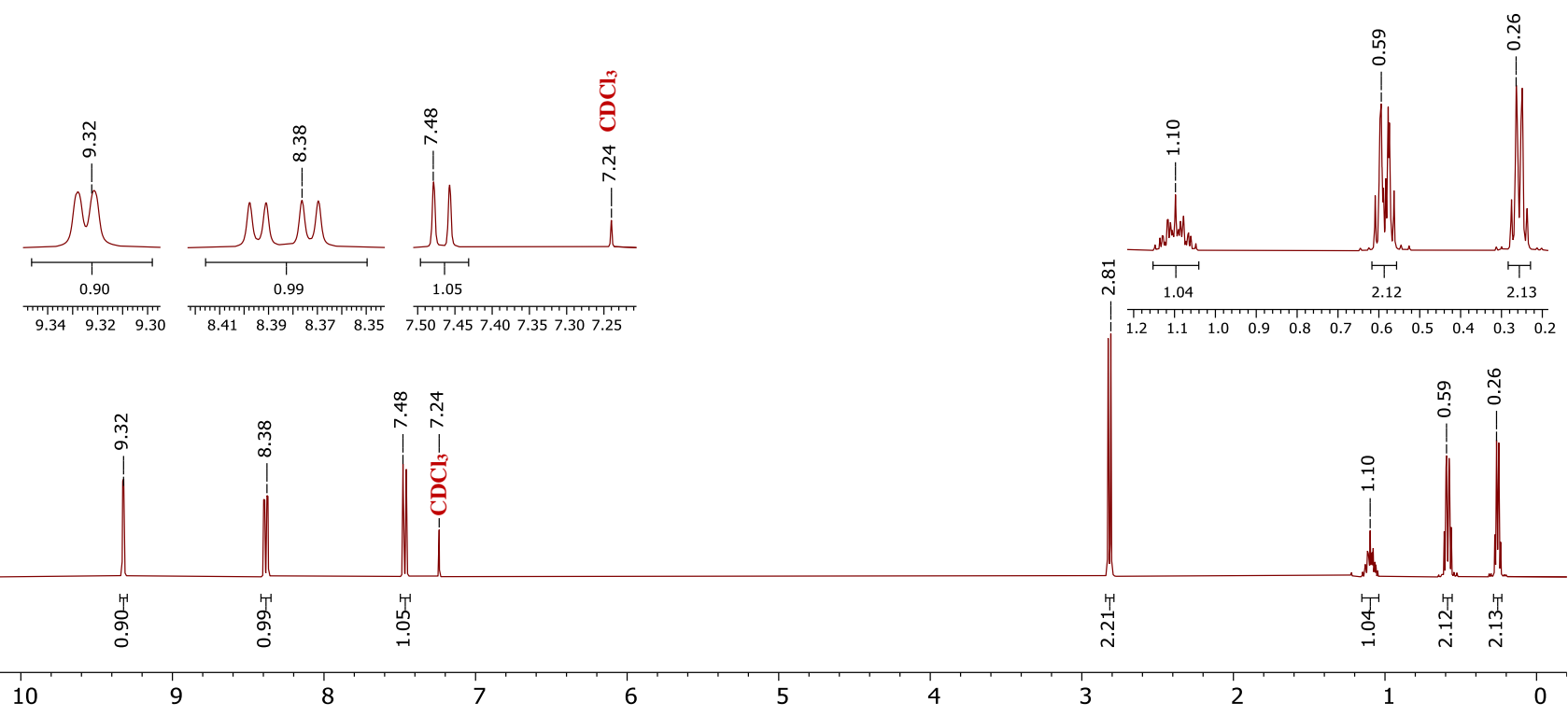

${ }^{13} \mathrm{C}$ NMR (100 MHz, $\left.\mathrm{CDCl}_{3}\right)$

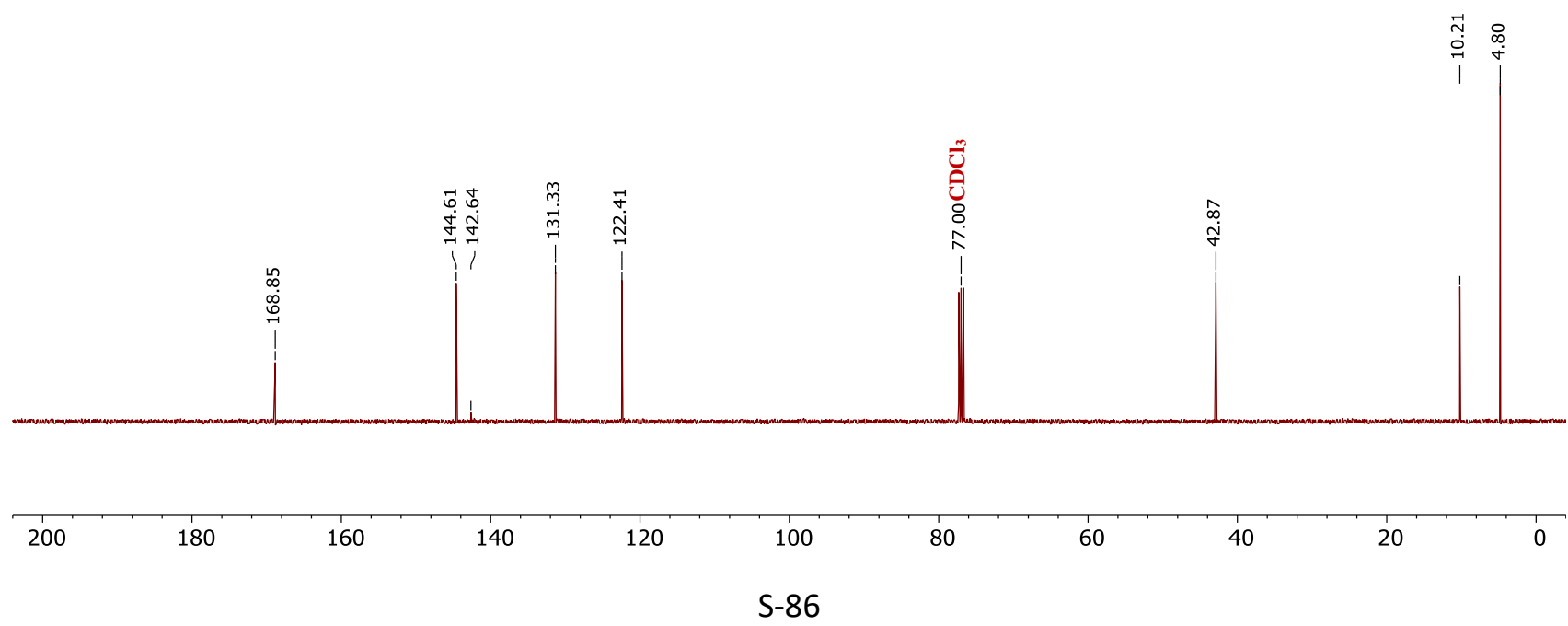




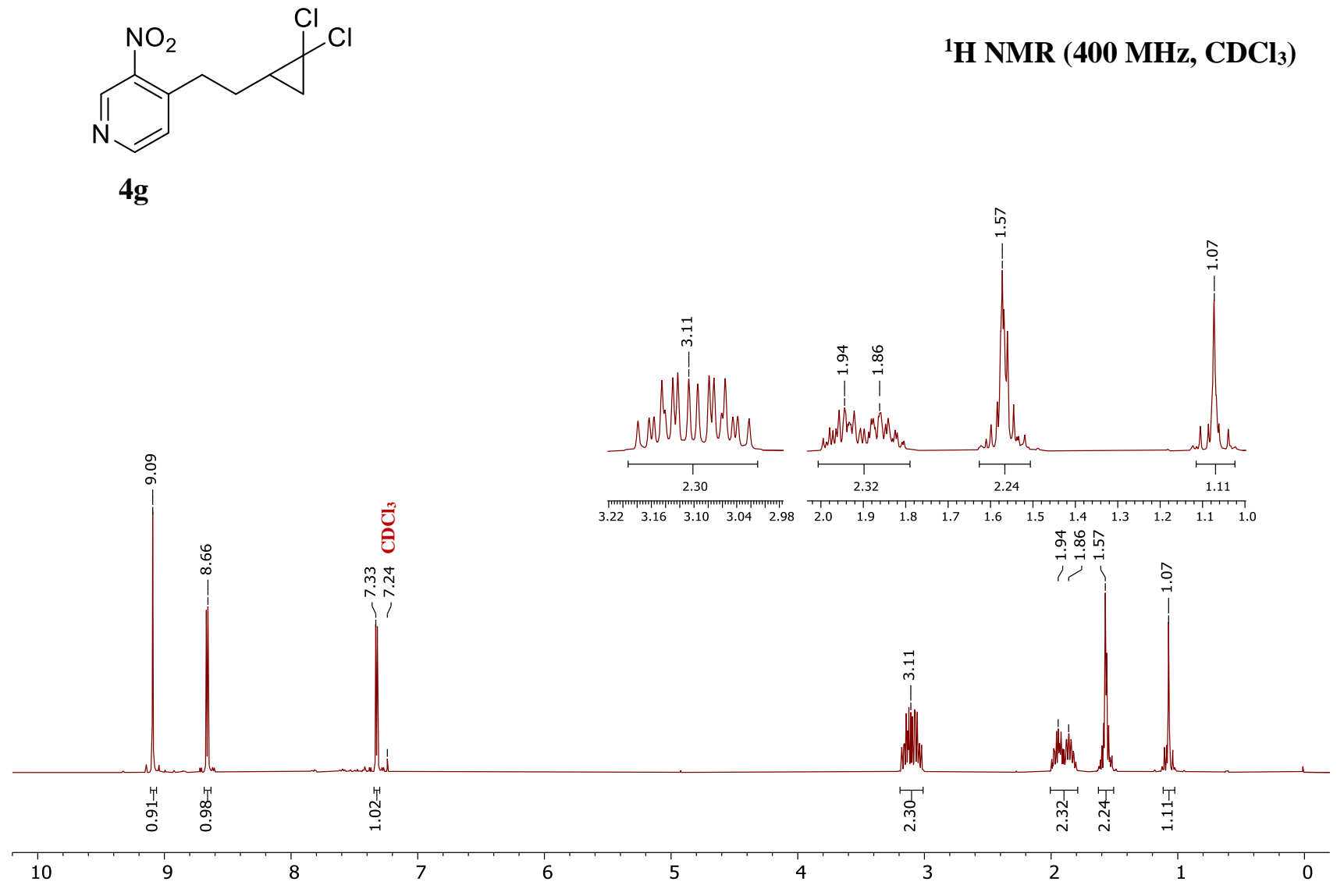

${ }^{13} \mathrm{C}$ NMR (100 MHz, $\left.\mathrm{CDCl}_{3}\right)$

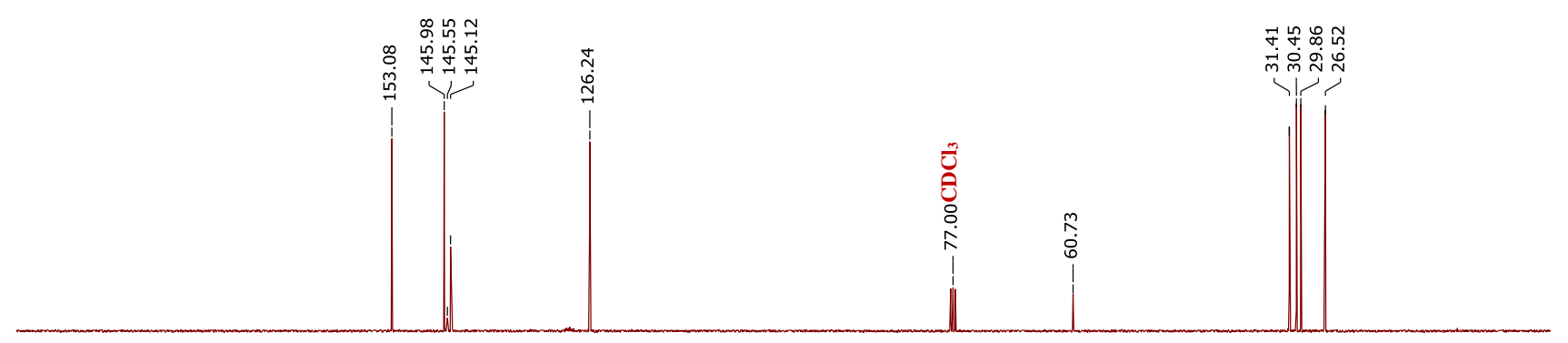


<smiles>CN1CCC(Cc2ccncc2[N+](=O)[O-])CC1</smiles>

4h

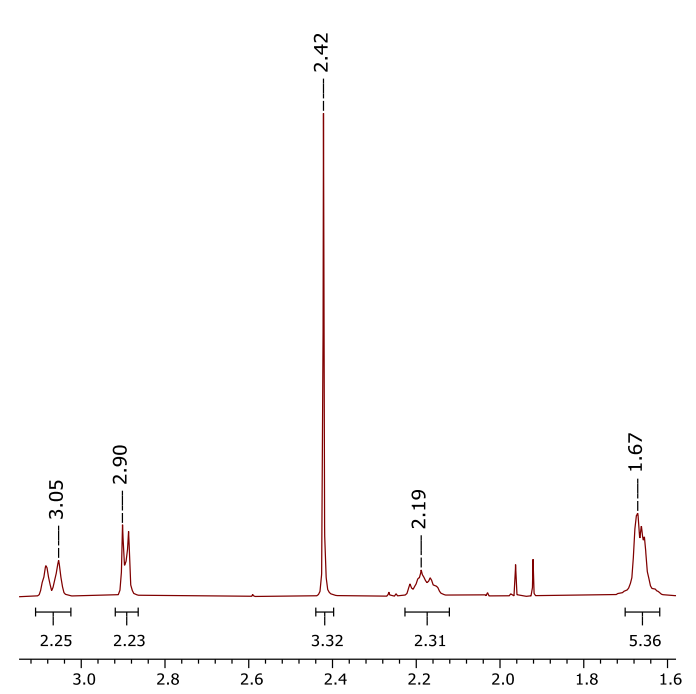

${ }^{1} \mathrm{H}$ NMR (400 MHz, $\left.\mathrm{CDCl}_{3}\right)$

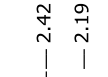

i
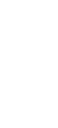


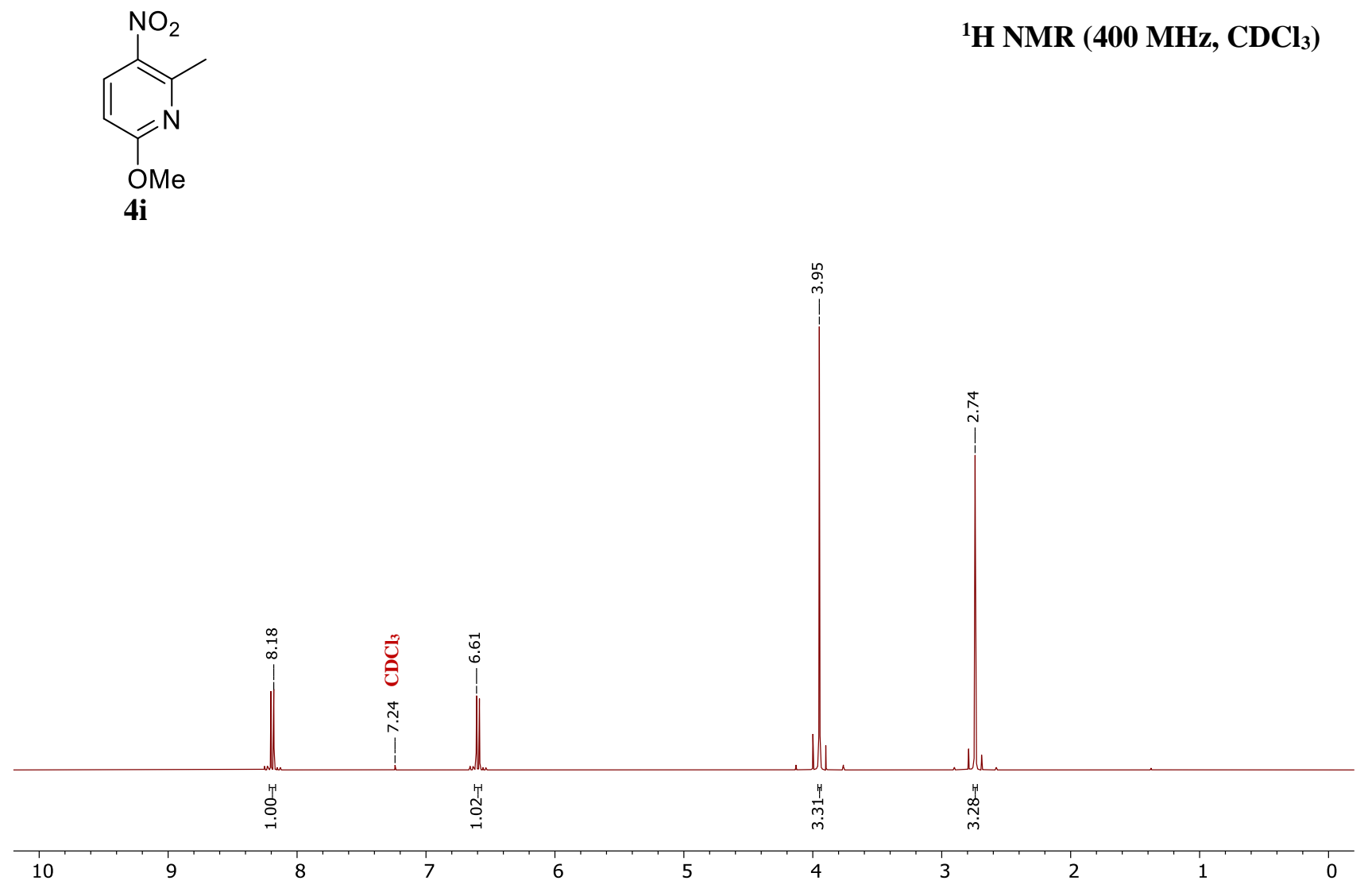

${ }^{13} \mathrm{C}$ NMR (100 MHz, $\left.\mathrm{CDCl}_{3}\right)$

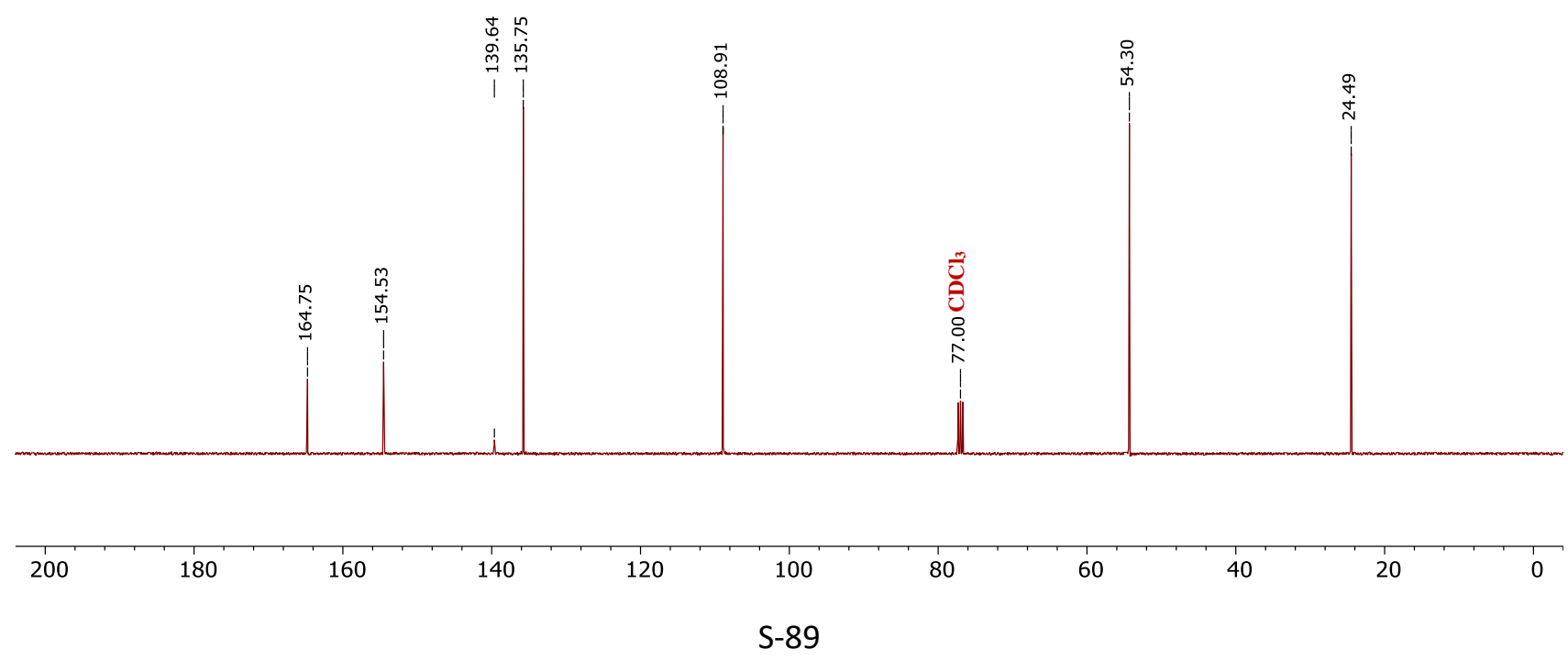




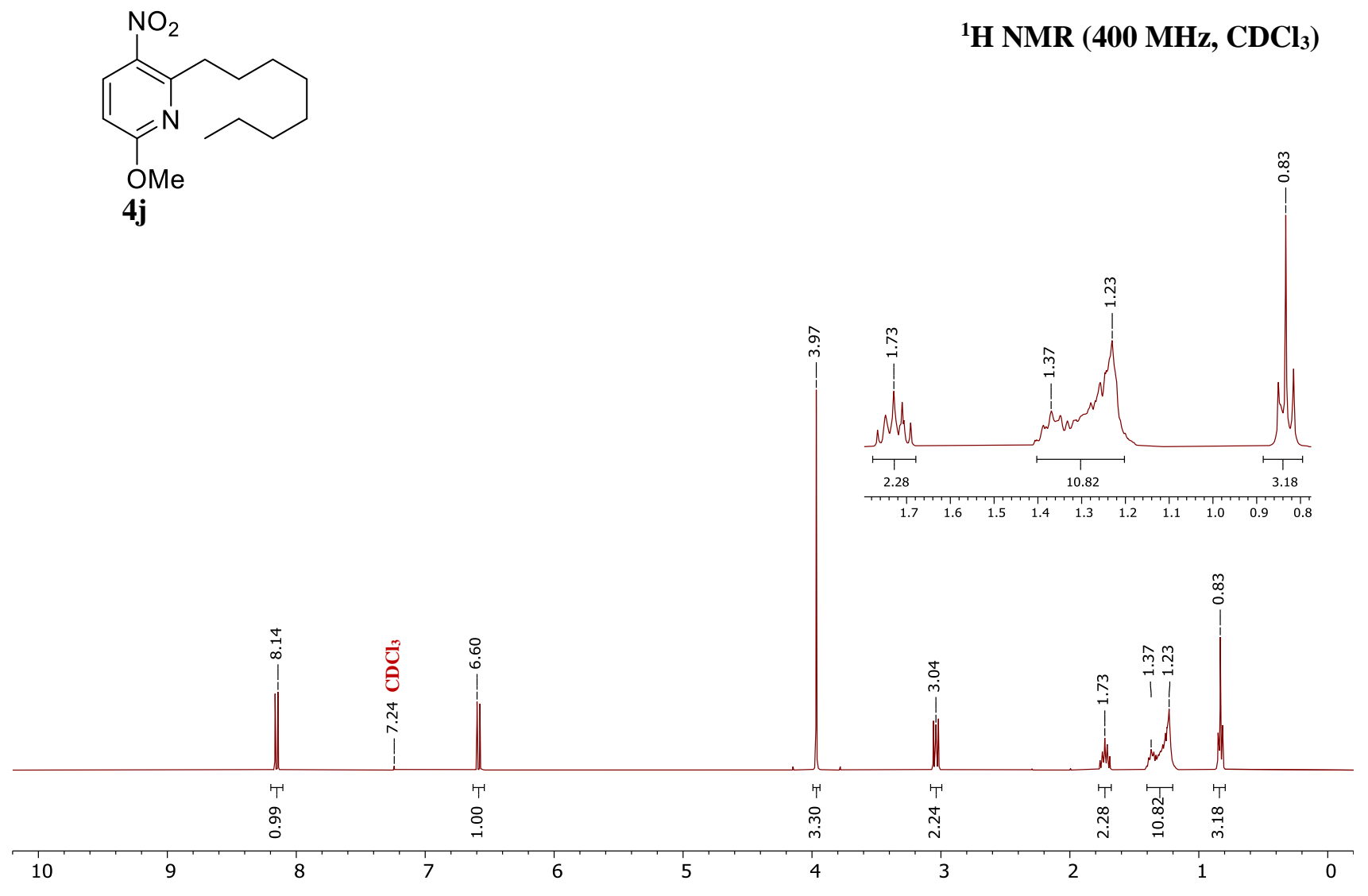

${ }^{13} \mathrm{C}$ NMR (100 MHz, $\left.\mathrm{CDCl}_{3}\right)$

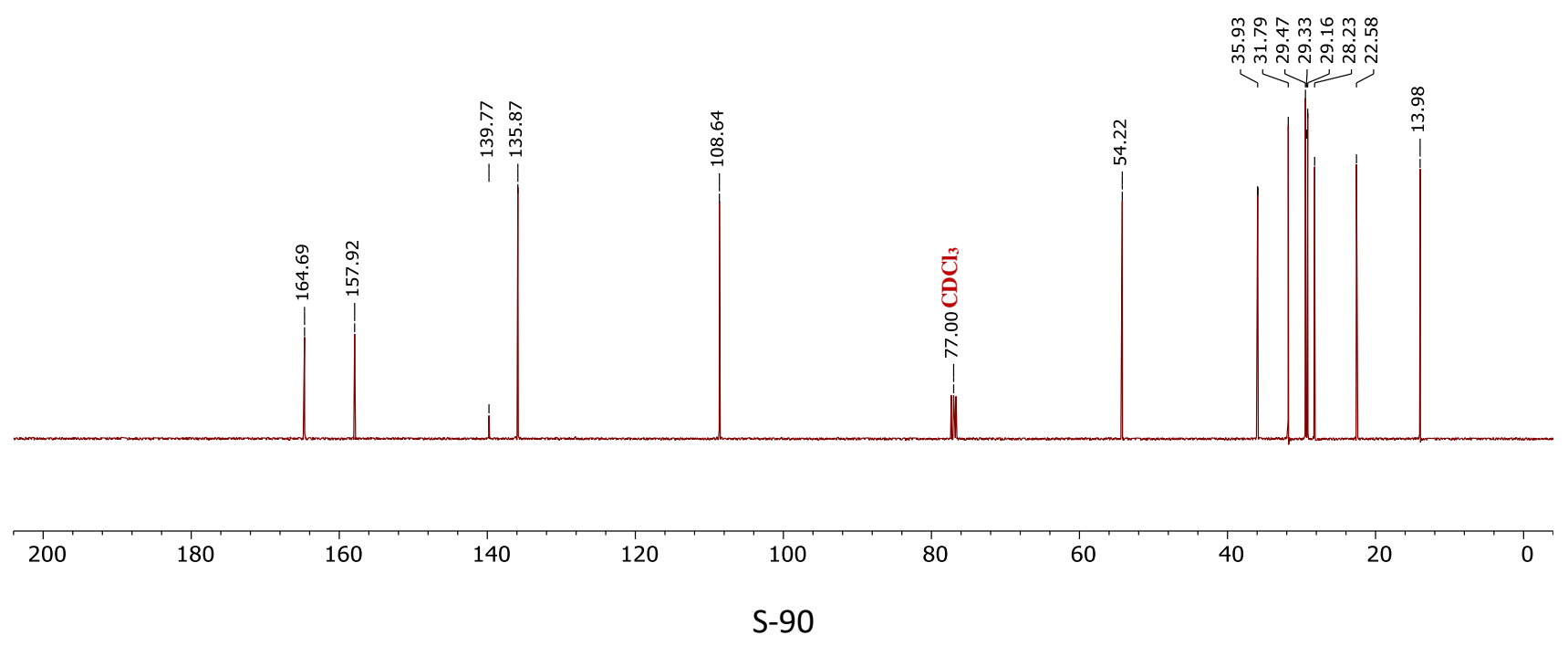


<smiles>CCCCCCCCc1cc(OC)c([N+](=O)[O-])cn1</smiles>

$4 k$

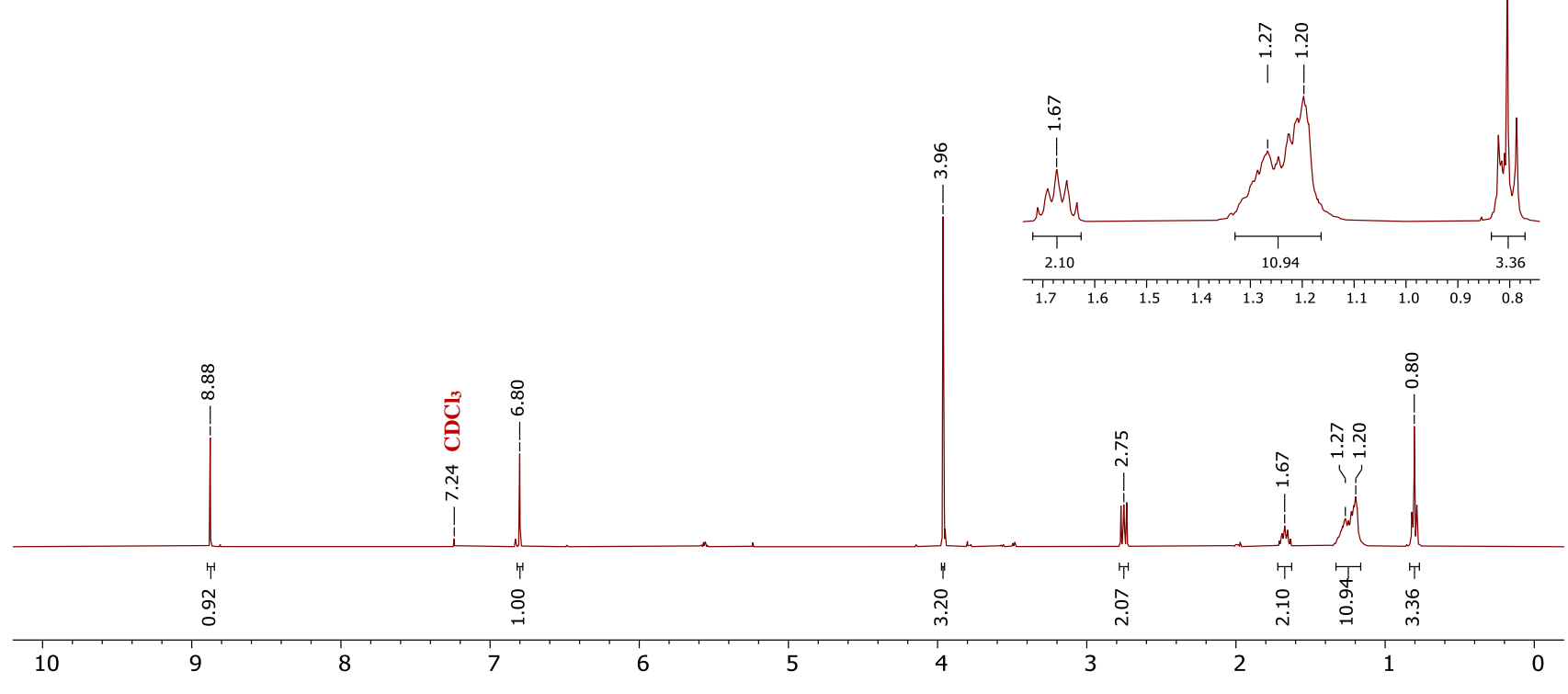

${ }^{13} \mathrm{C}$ NMR (100 MHz, $\left.\mathrm{CDCl}_{3}\right)$
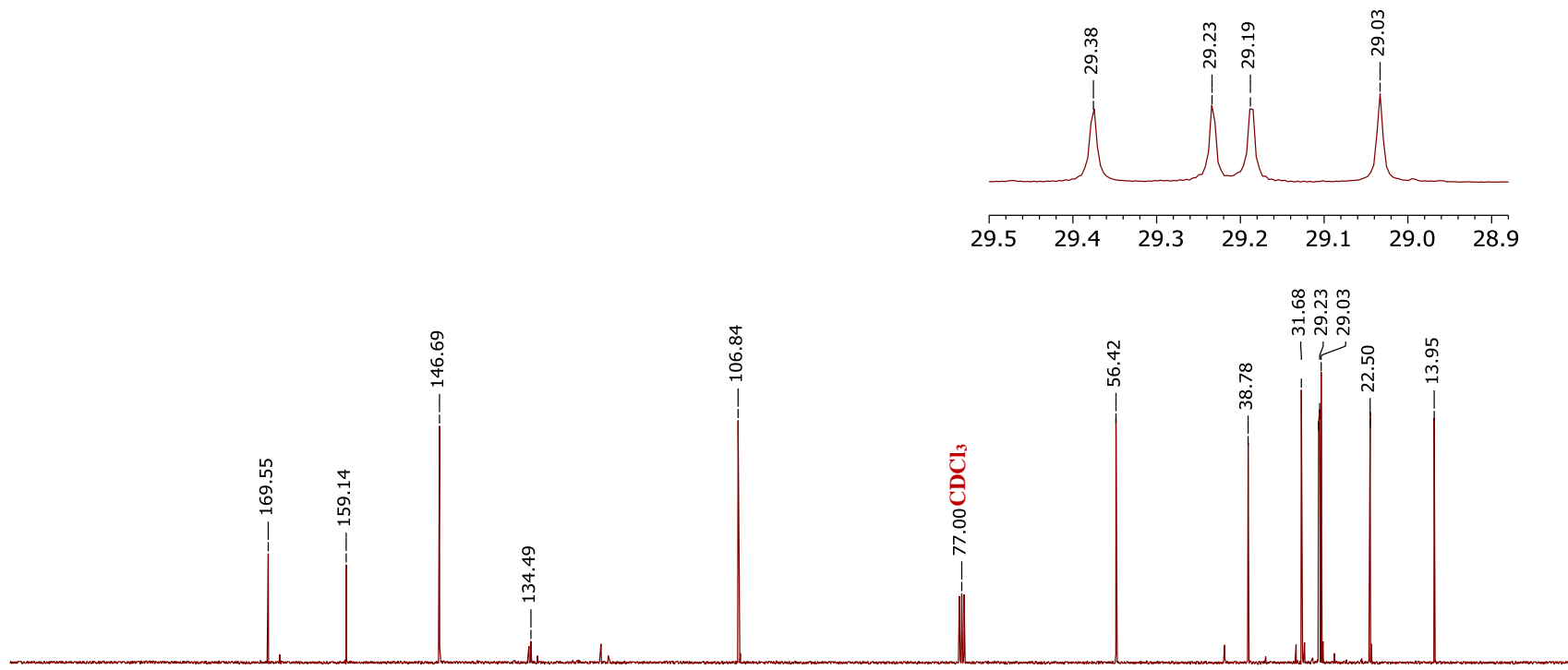


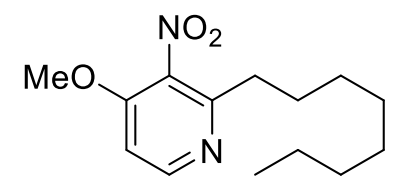

$4 k^{\prime}$

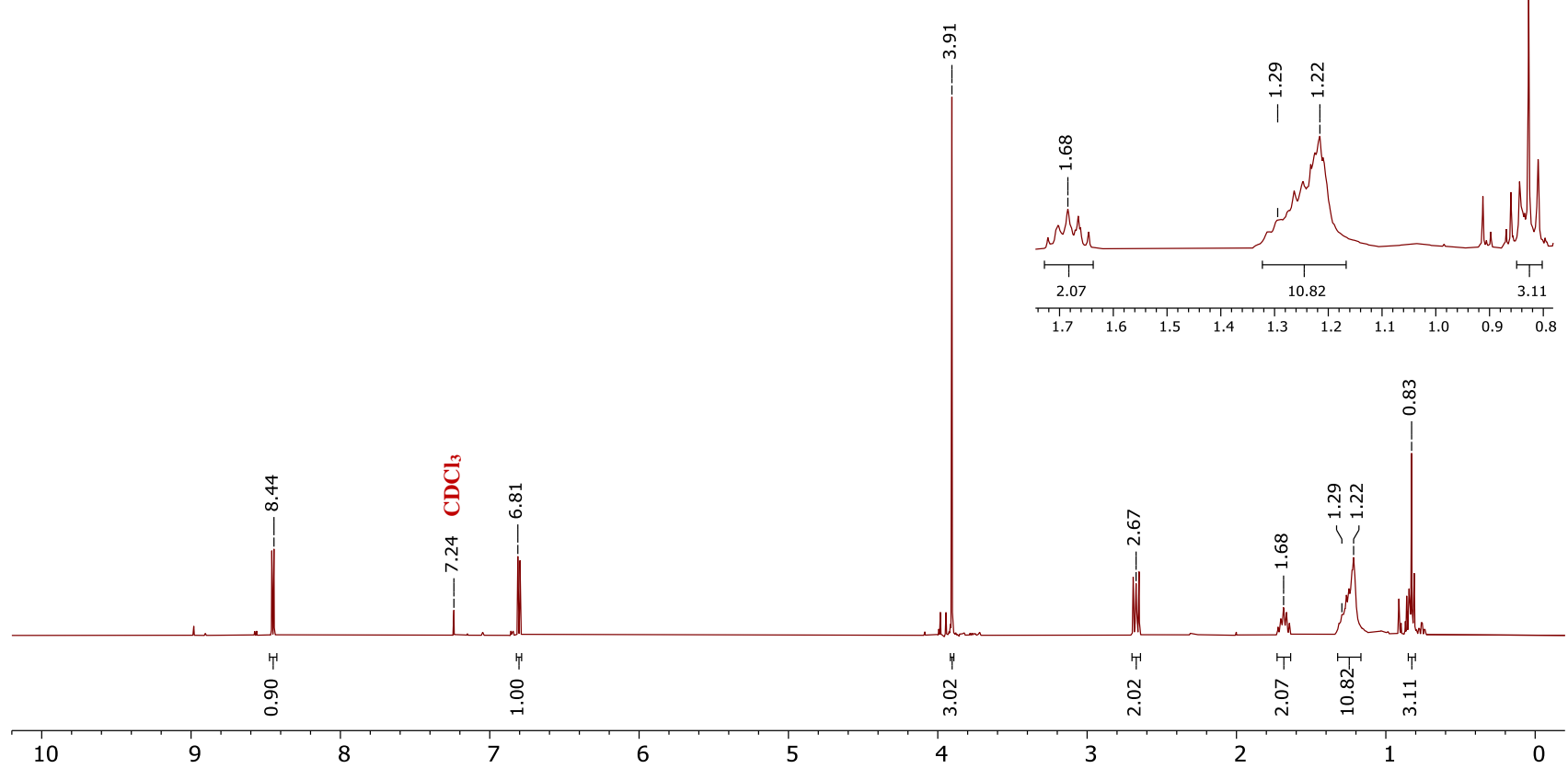

${ }^{13} \mathrm{C}$ NMR (100 MHz, $\left.\mathrm{CDCl}_{3}\right)$

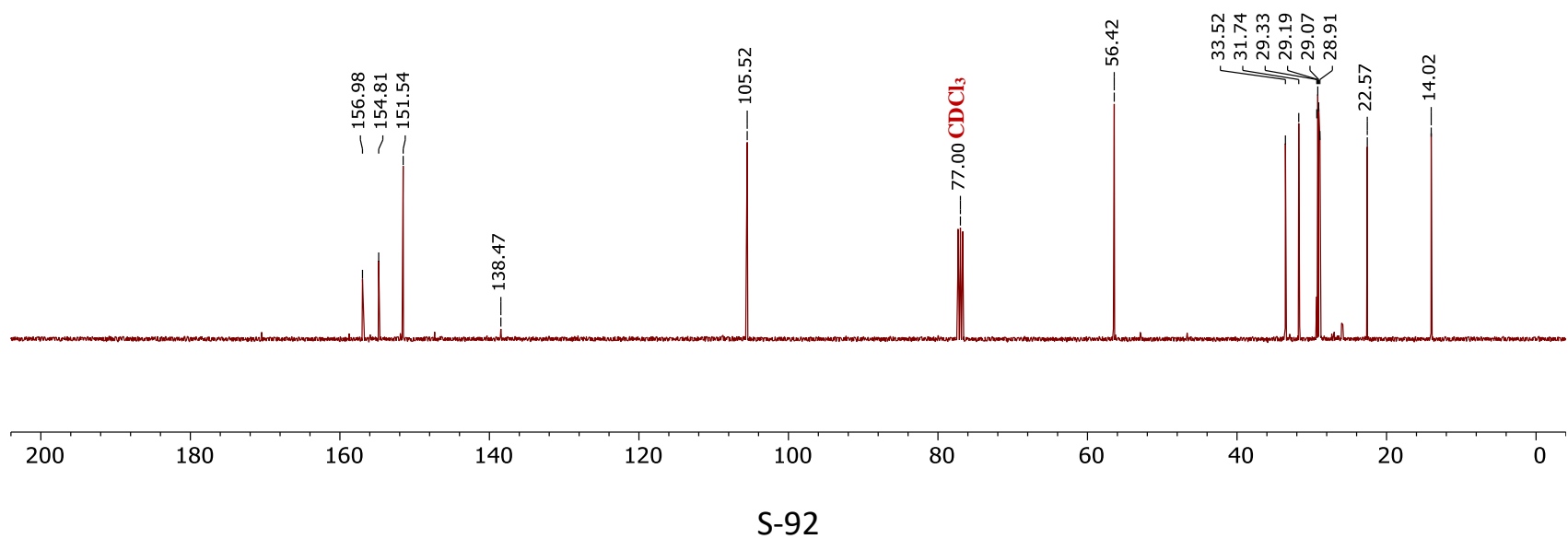




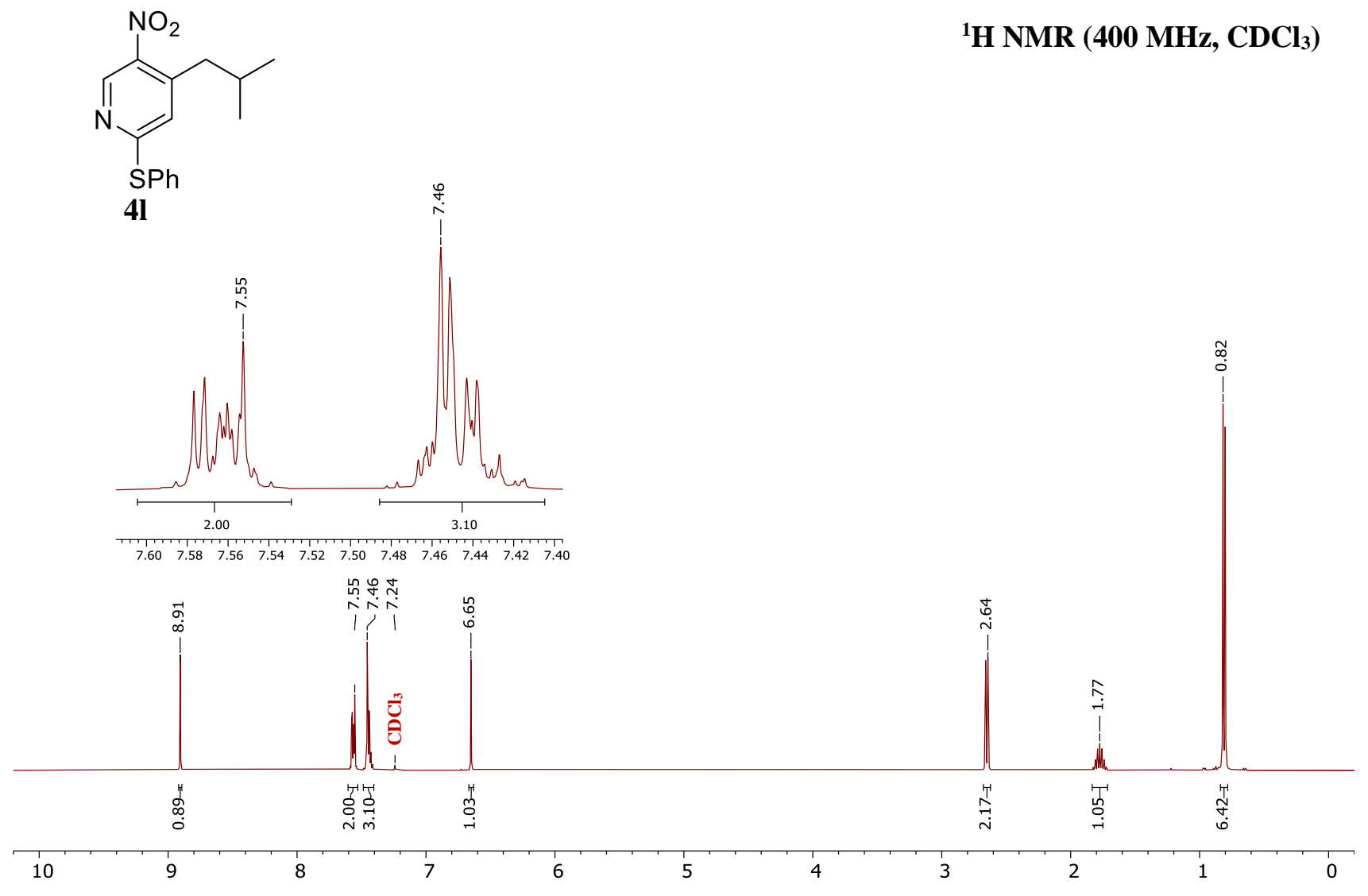

${ }^{13} \mathrm{C}$ NMR (100 MHz, $\left.\mathrm{CDCl}_{3}\right)$

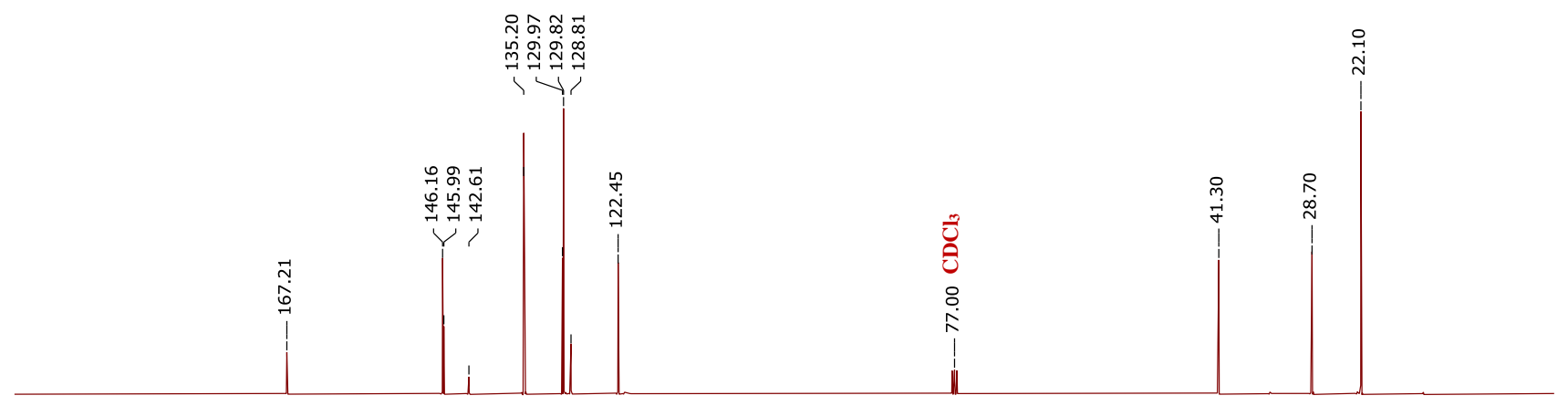




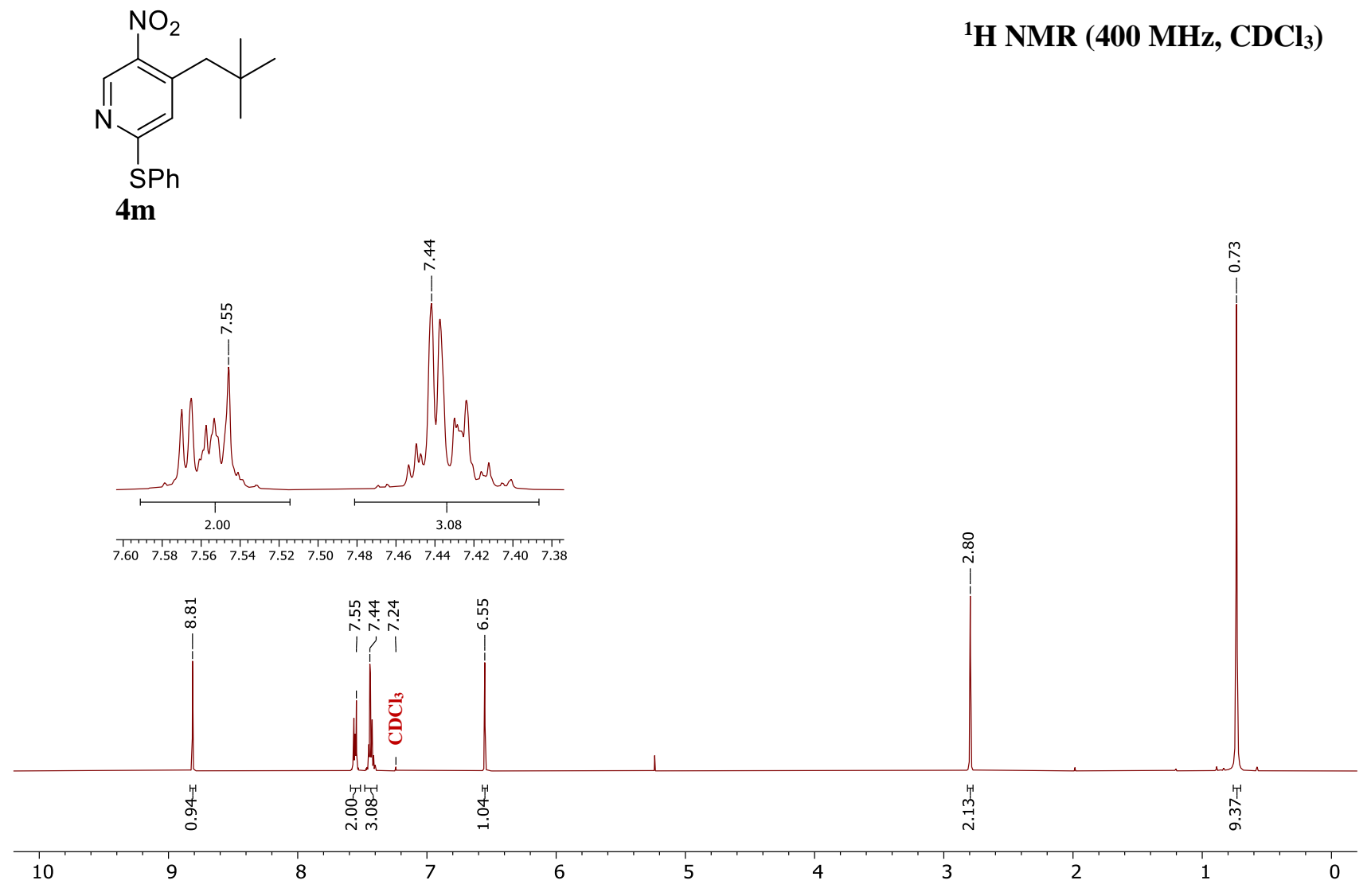

${ }^{13} \mathrm{C}$ NMR (100 MHz, $\left.\mathrm{CDCl}_{3}\right)$

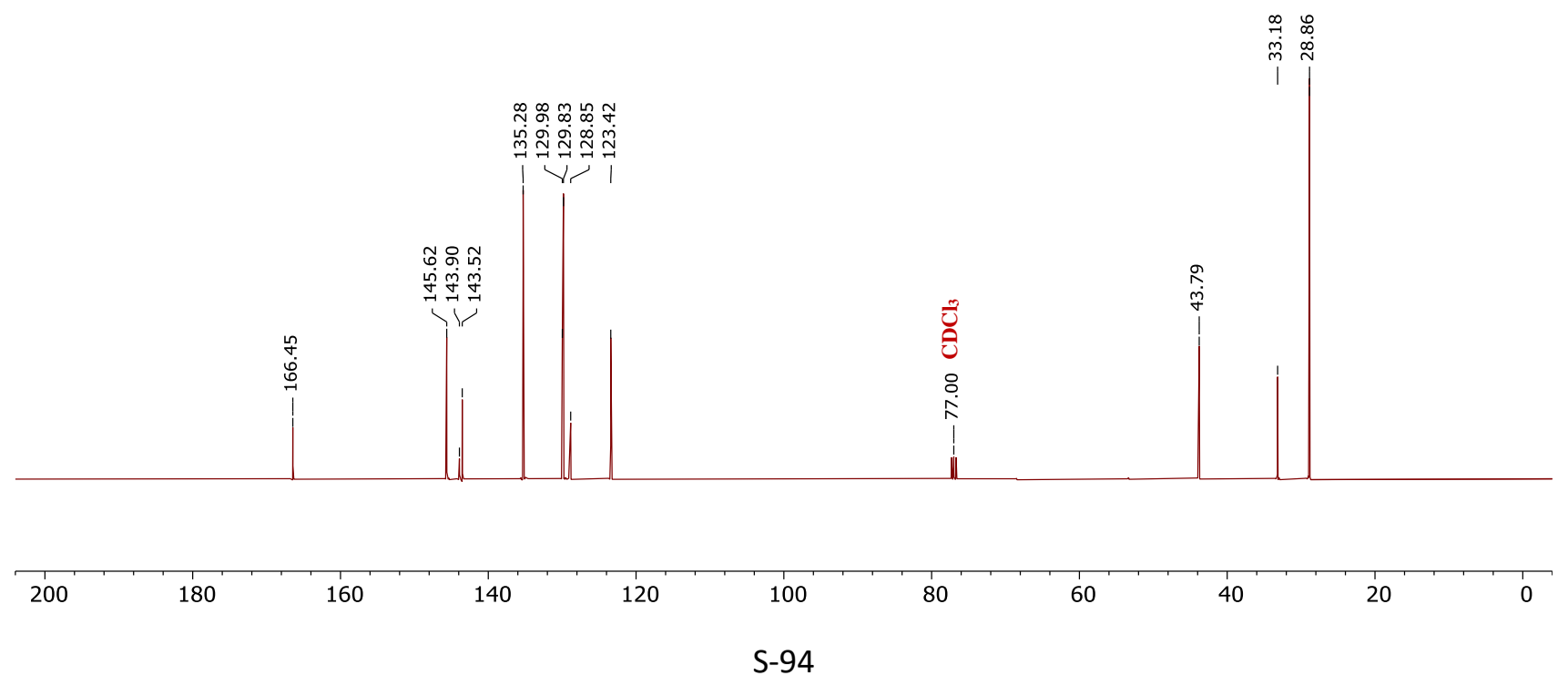


<smiles>CC(C)(C)Cc1nc([Sb]2[CH][CH]2)ccc1[N+](=O)[O-]</smiles>

${ }^{1} \mathrm{H}$ NMR (400 MHz, $\mathrm{CDCl}_{3}$ )
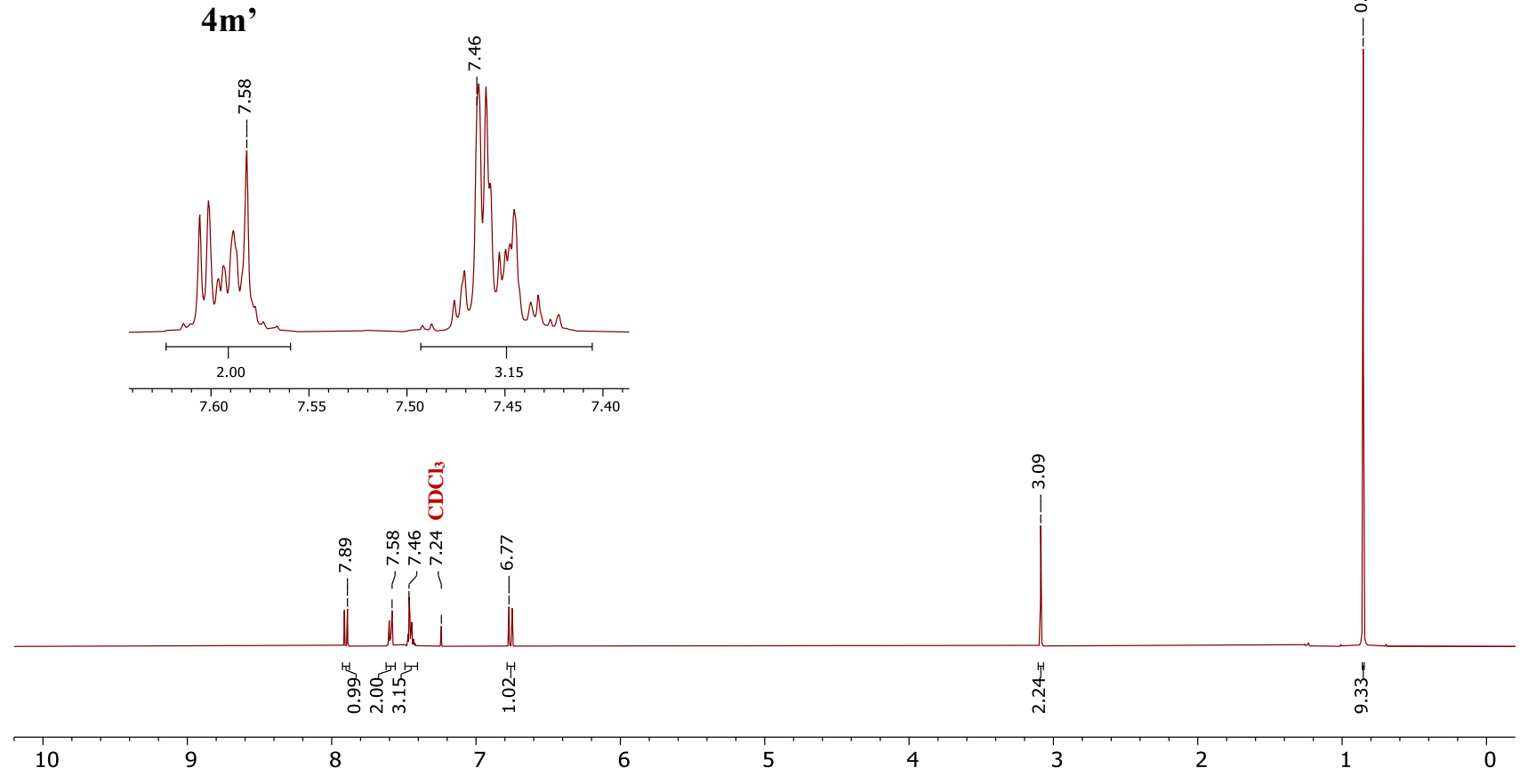

${ }^{13} \mathrm{C}$ NMR (100 MHz, $\left.\mathrm{CDCl}_{3}\right)$

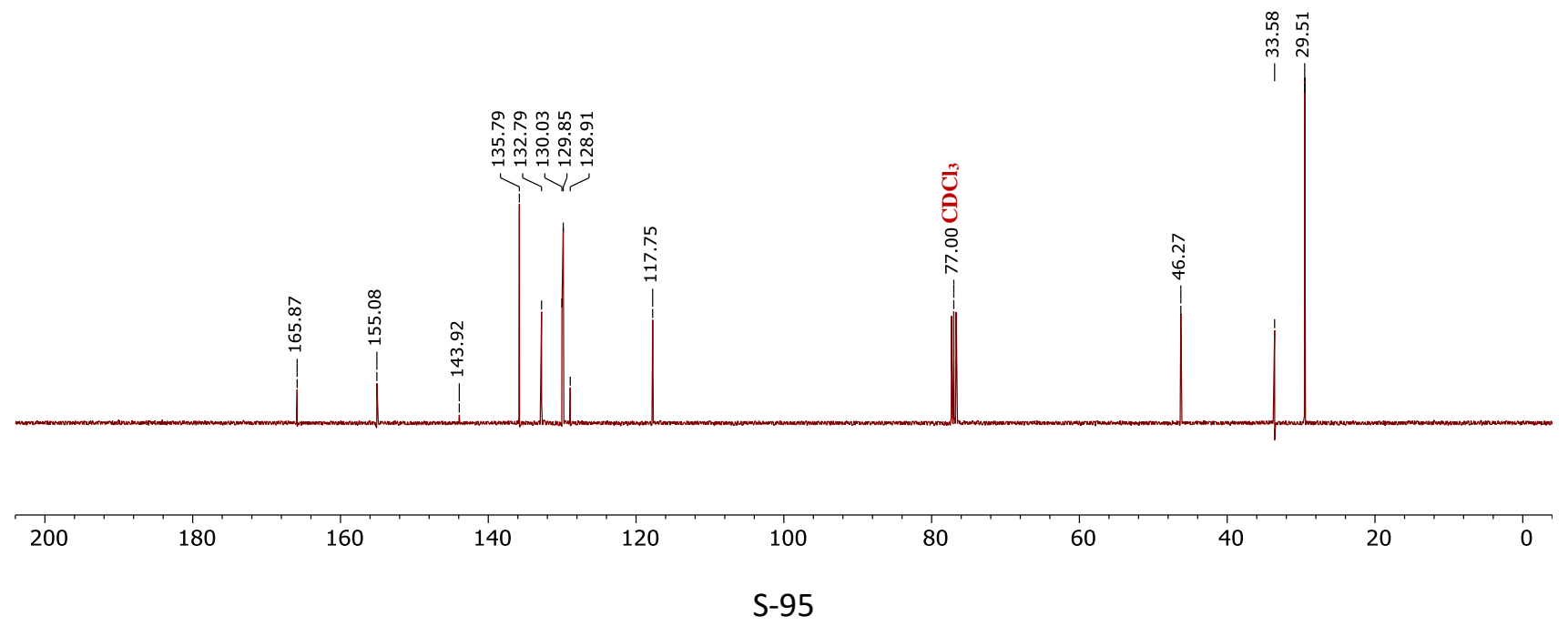




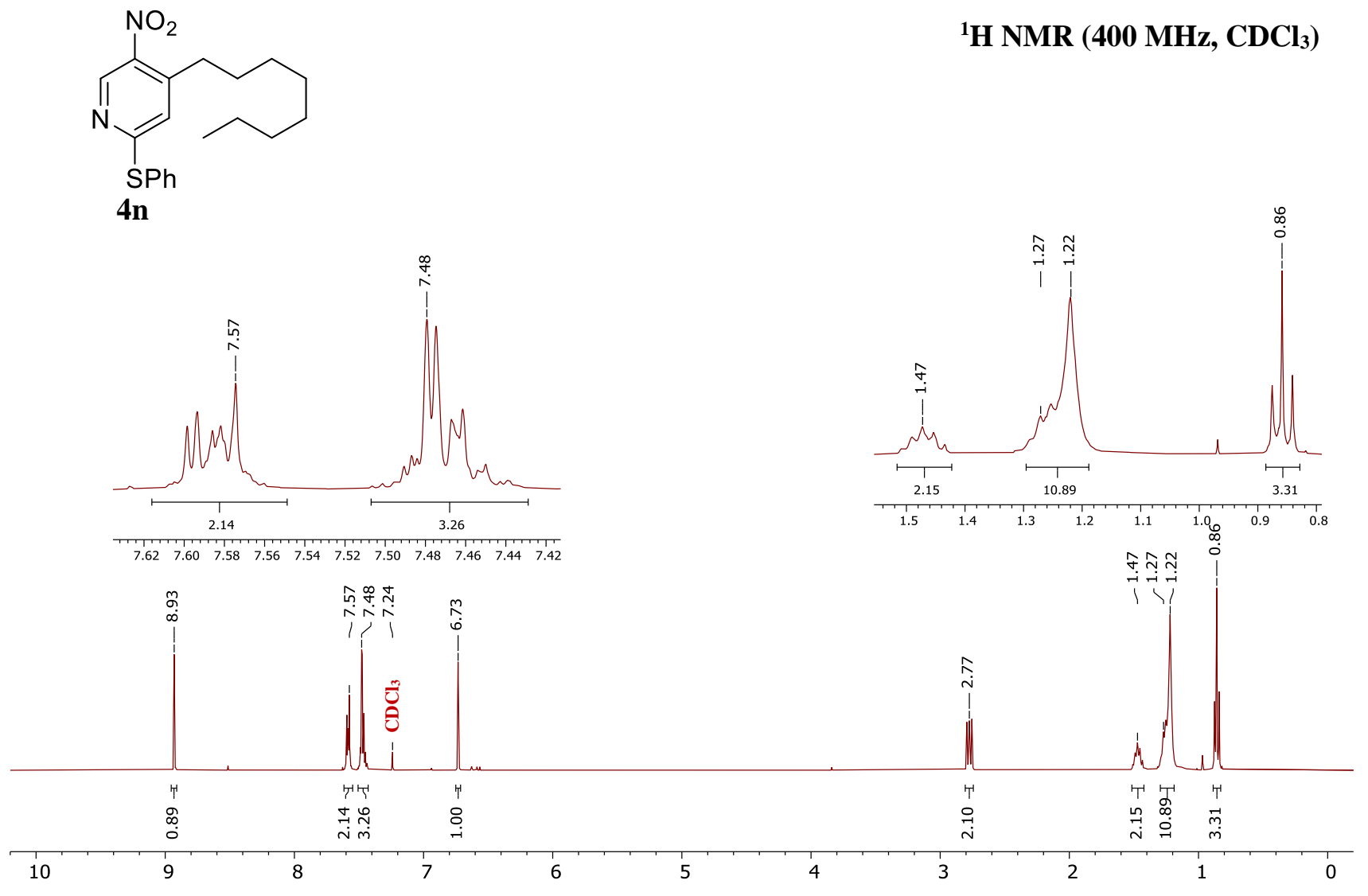

${ }^{13} \mathrm{C}$ NMR (100 MHz, $\left.\mathrm{CDCl}_{3}\right)$

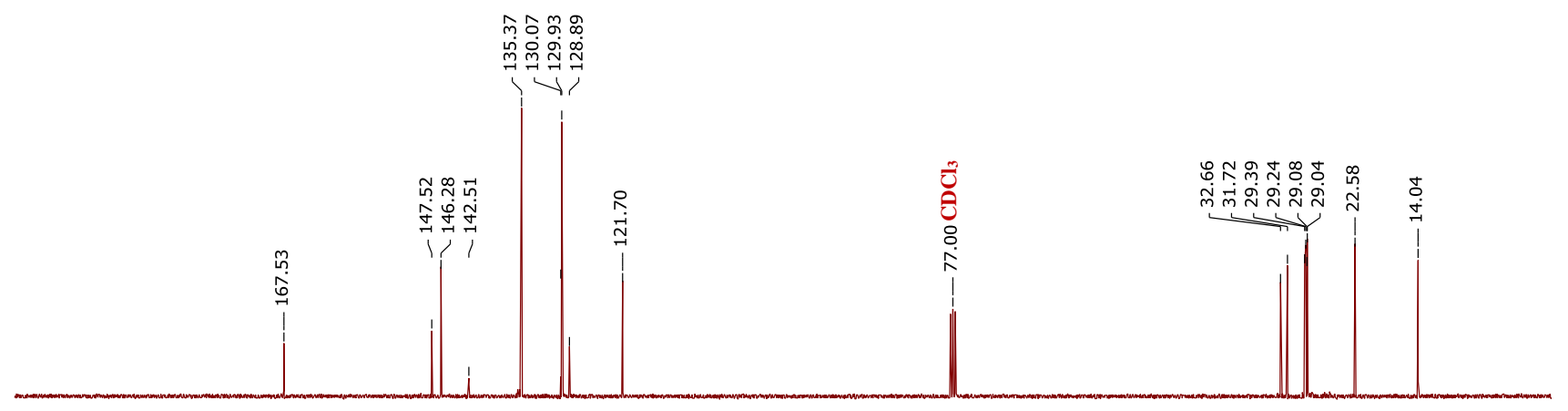




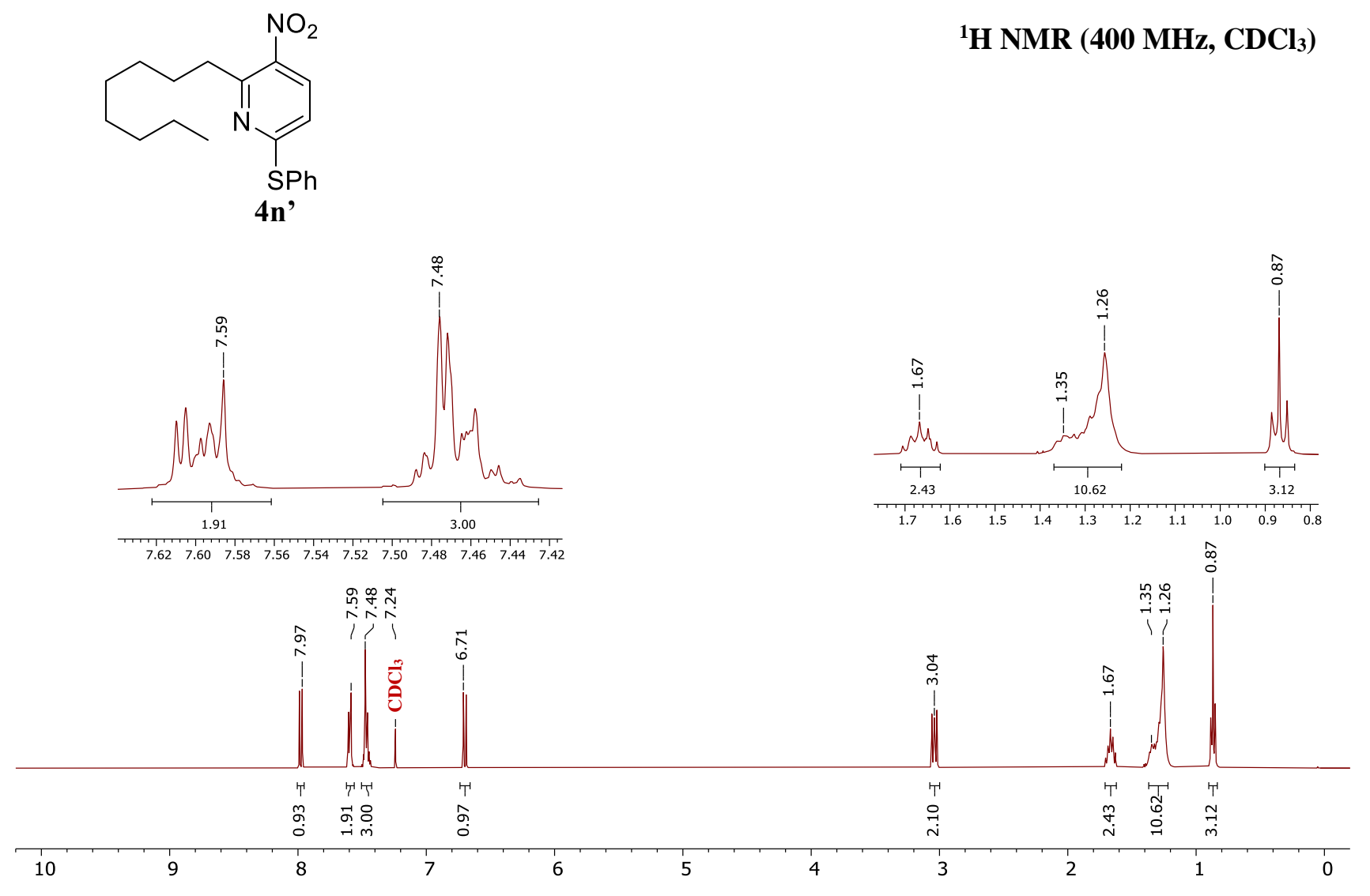

${ }^{13} \mathrm{C}$ NMR (100 MHz, $\left.\mathrm{CDCl}_{3}\right)$

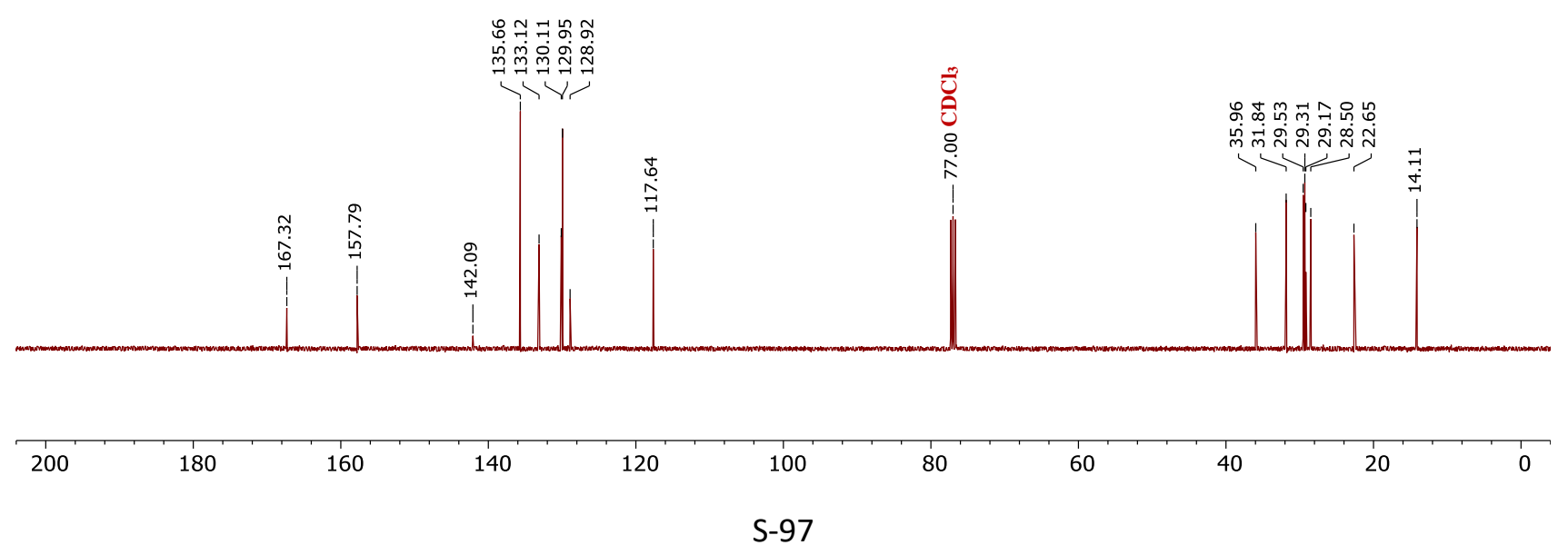




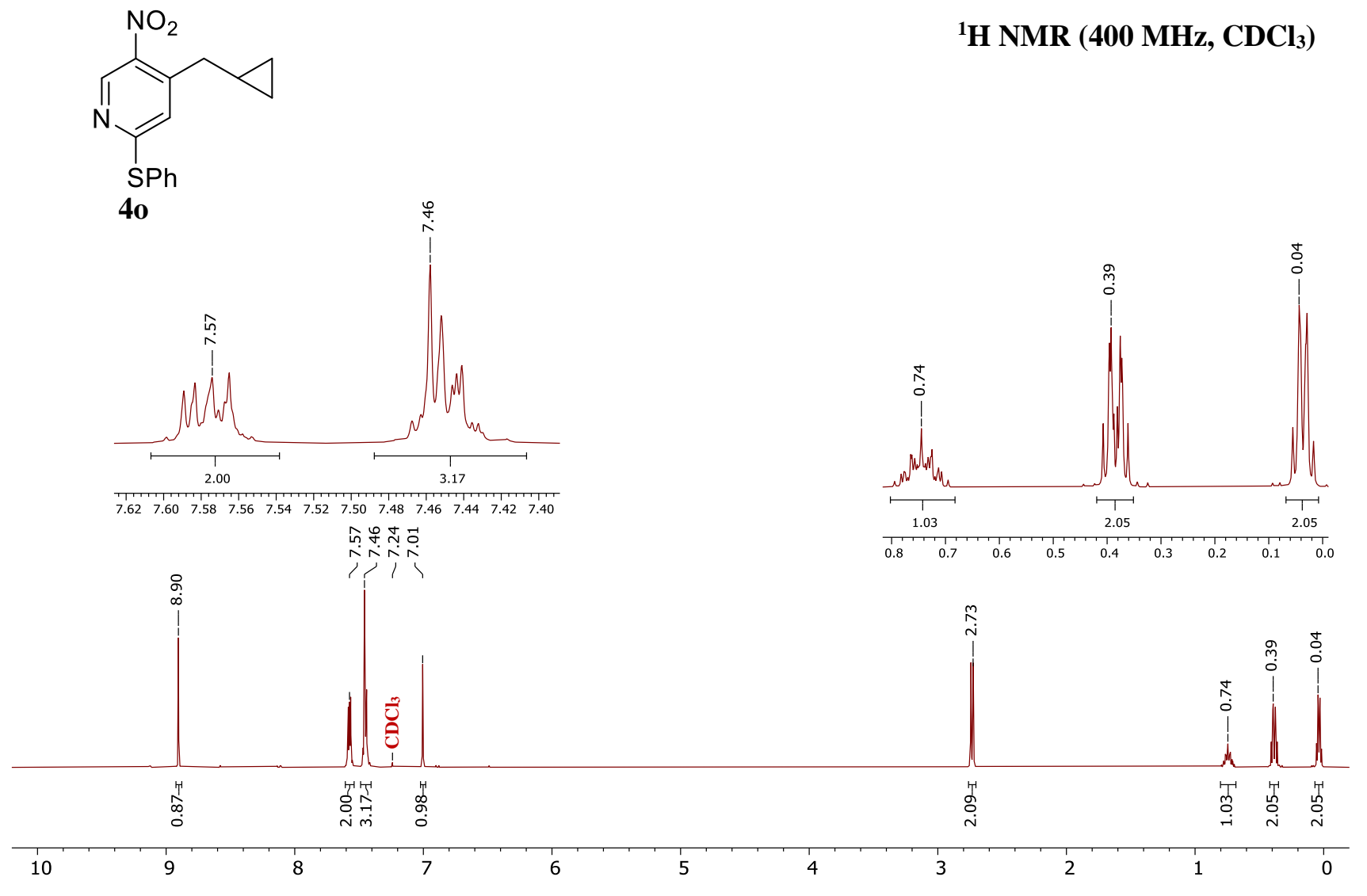

${ }^{13} \mathrm{C}$ NMR (100 MHz, $\left.\mathrm{CDCl}_{3}\right)$

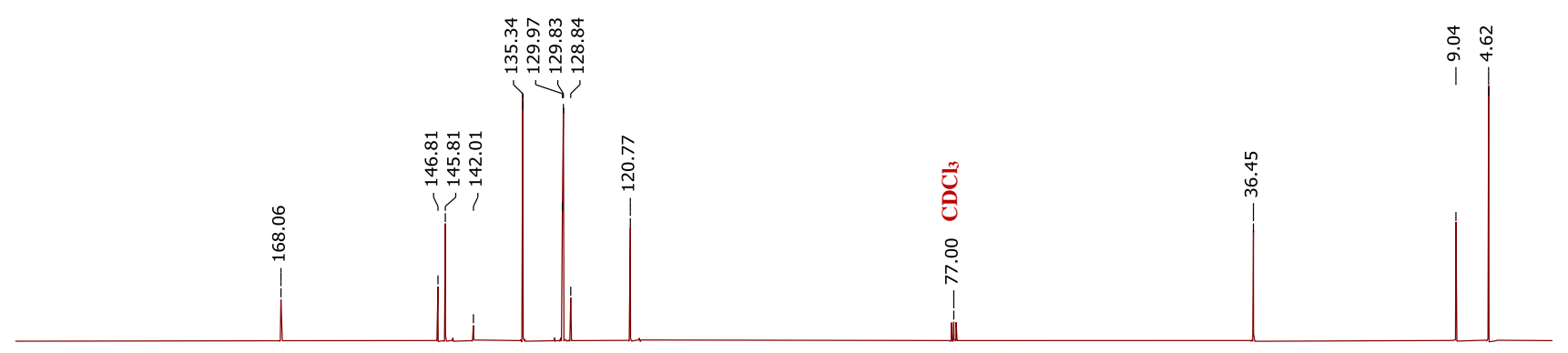

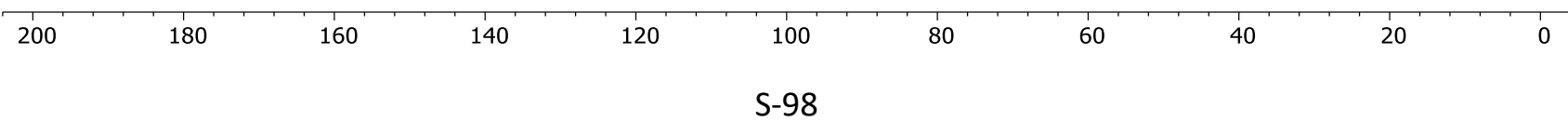




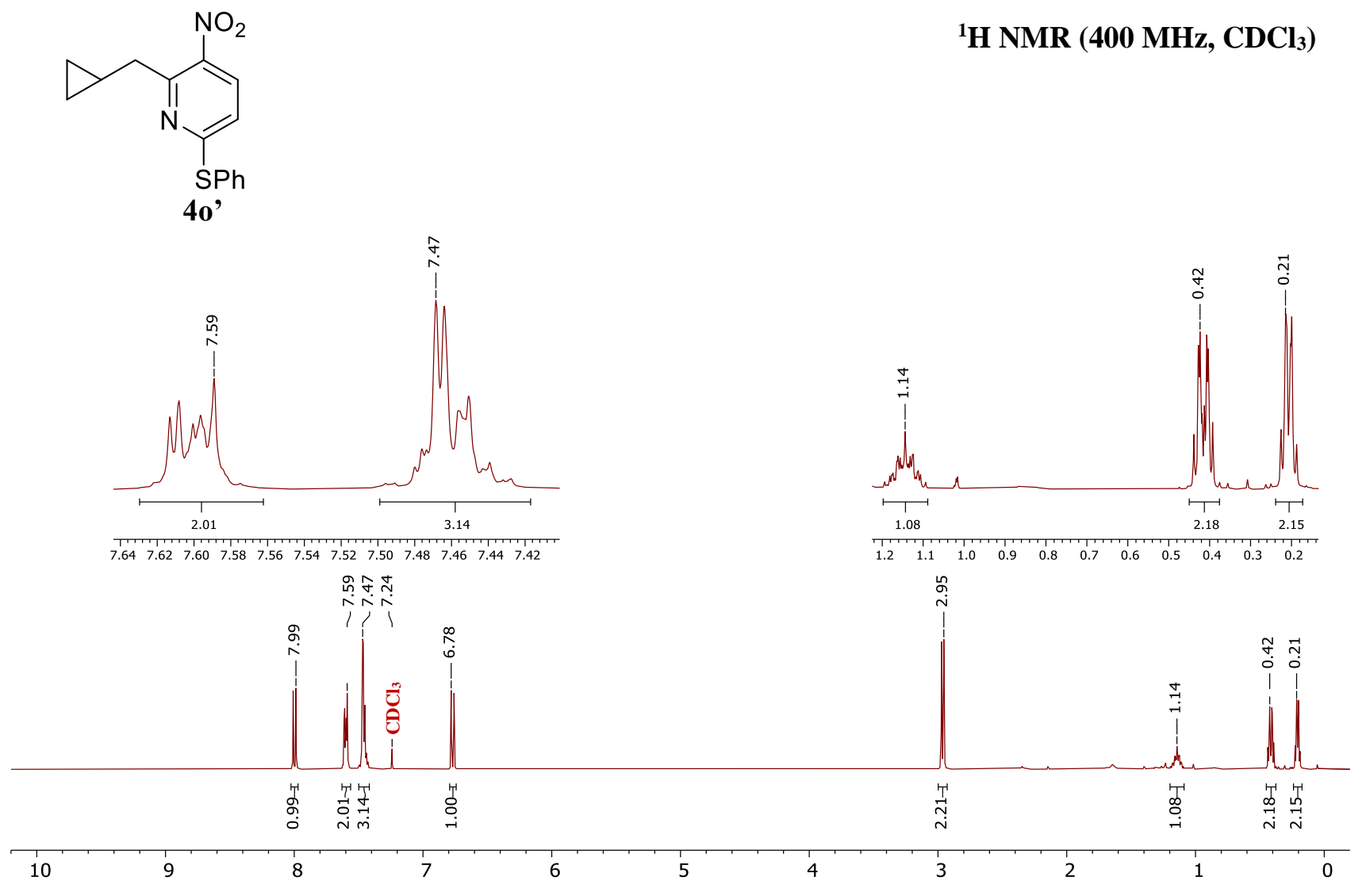

${ }^{13} \mathrm{C}$ NMR (100 MHz, $\left.\mathrm{CDCl}_{3}\right)$

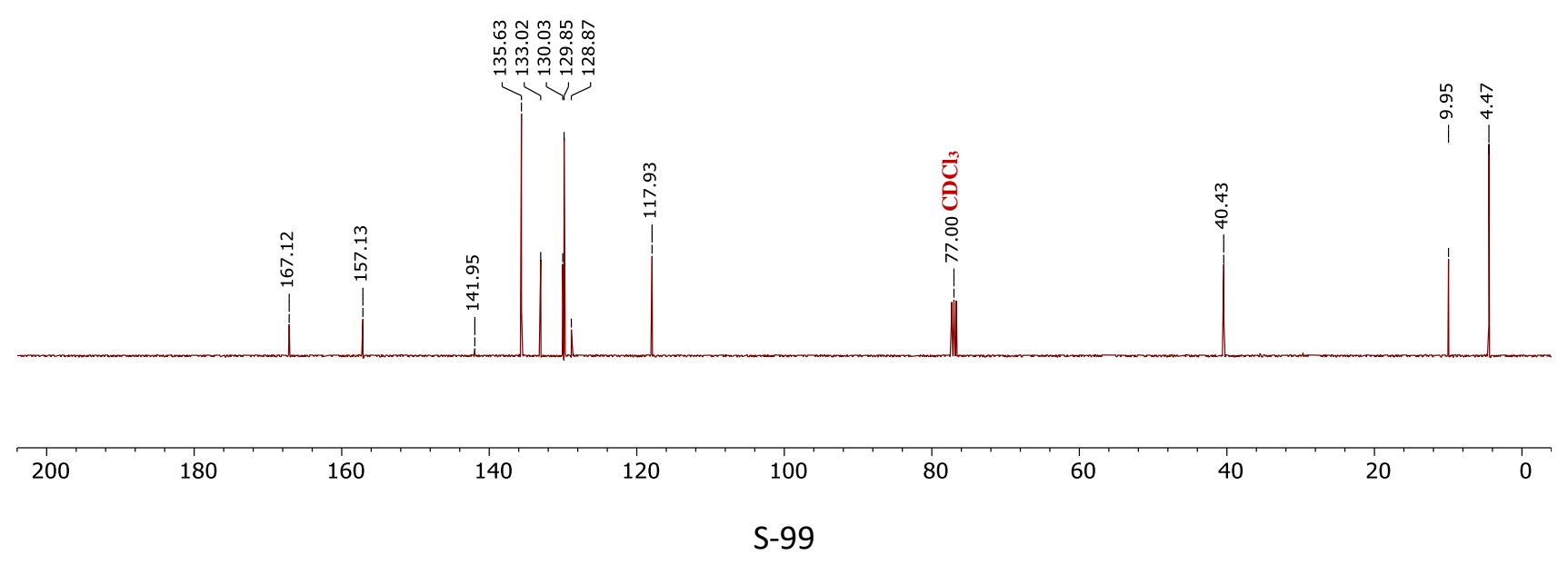




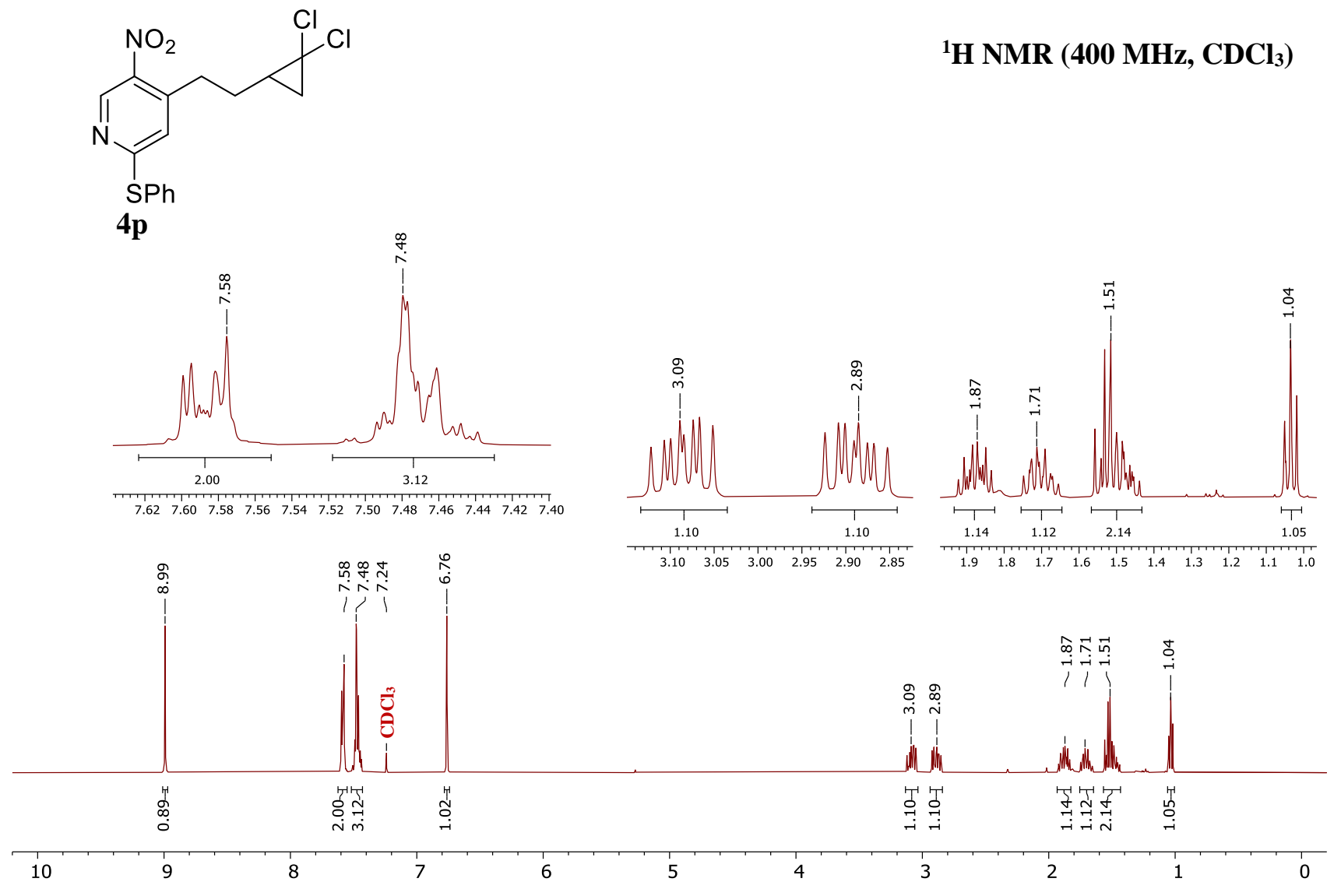

${ }^{13} \mathrm{C}$ NMR (100 MHz, $\left.\mathrm{CDCl}_{3}\right)$

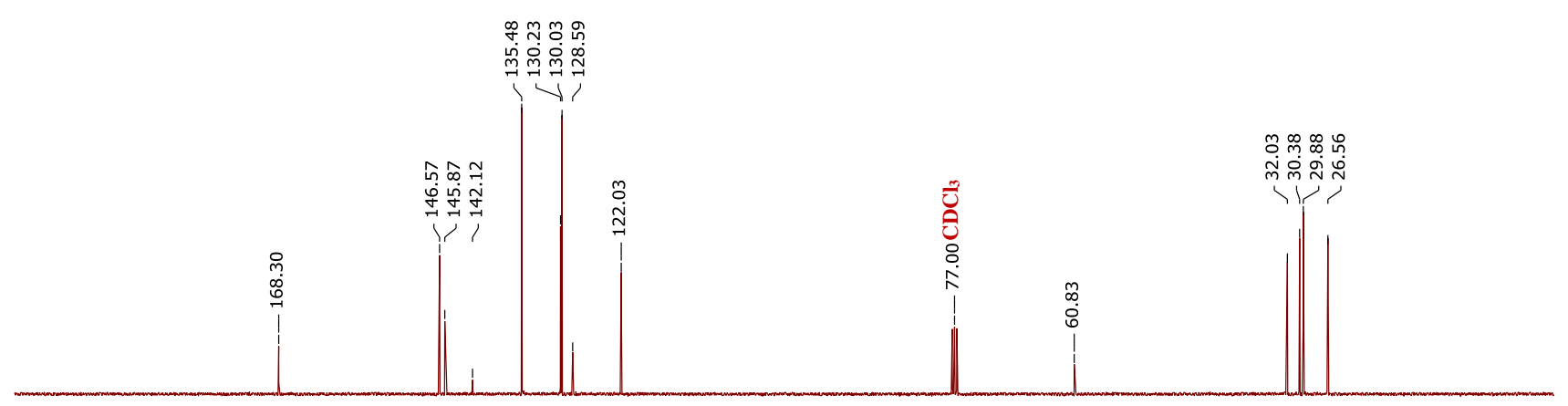




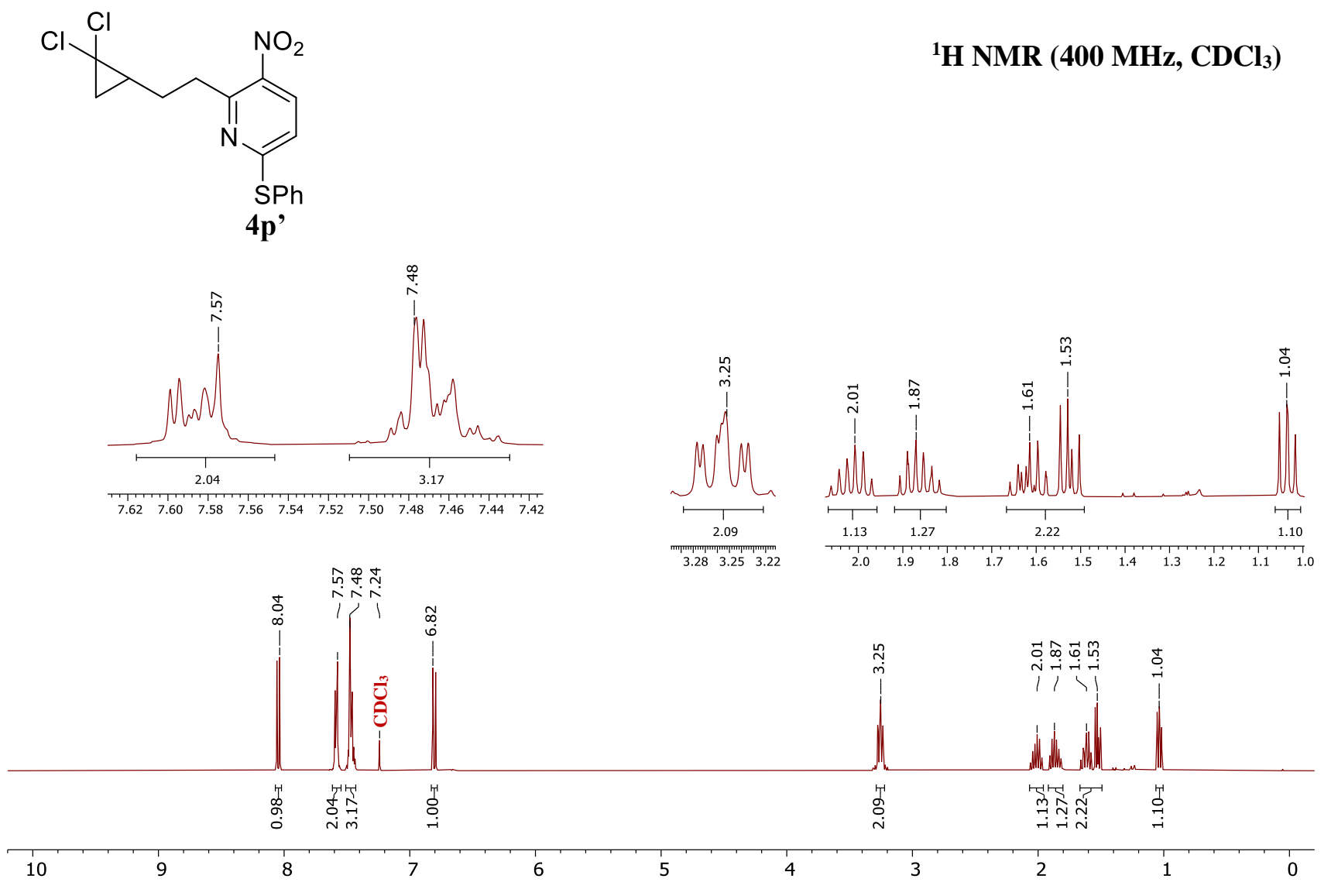

${ }^{13} \mathrm{C}$ NMR (100 $\left.\mathrm{MHz}, \mathrm{CDCl}_{3}\right)$

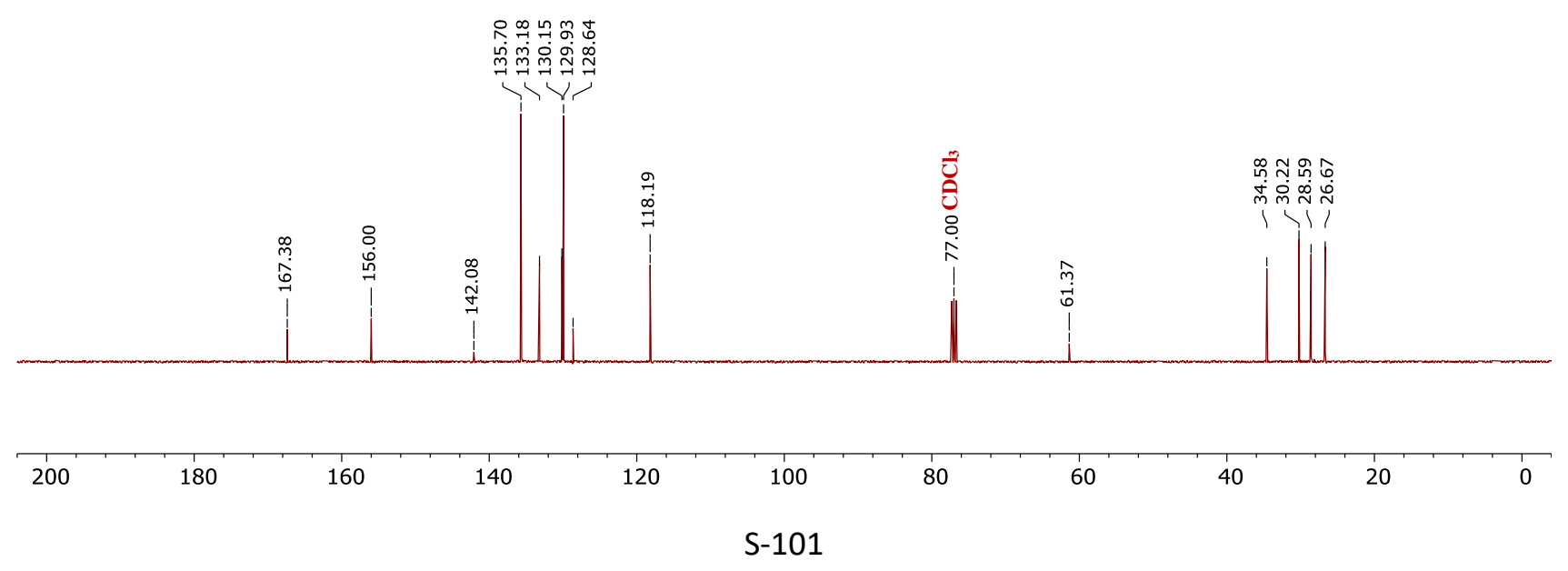




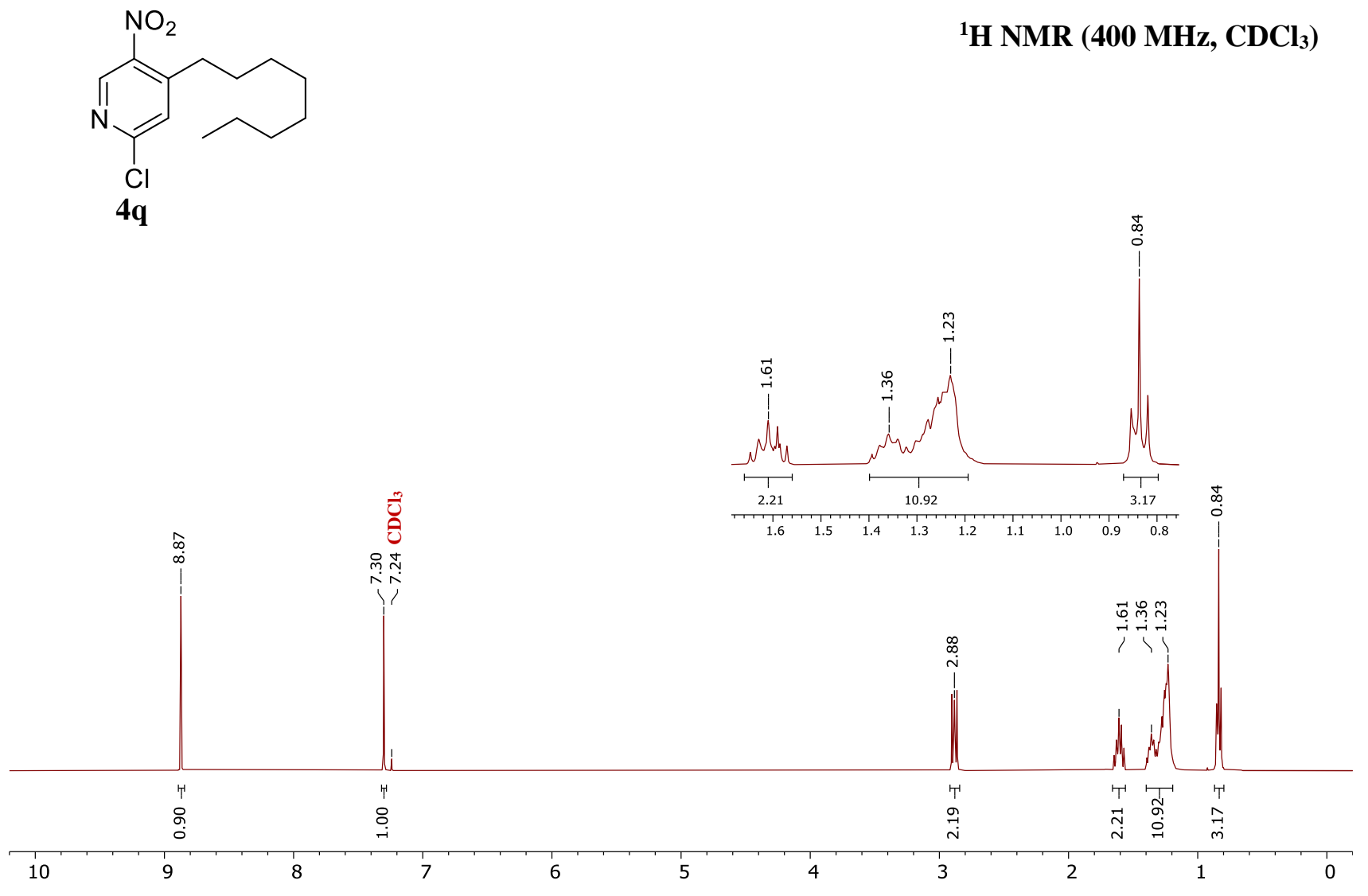

${ }^{13} \mathrm{C}$ NMR (100 MHz, $\left.\mathrm{CDCl}_{3}\right)$

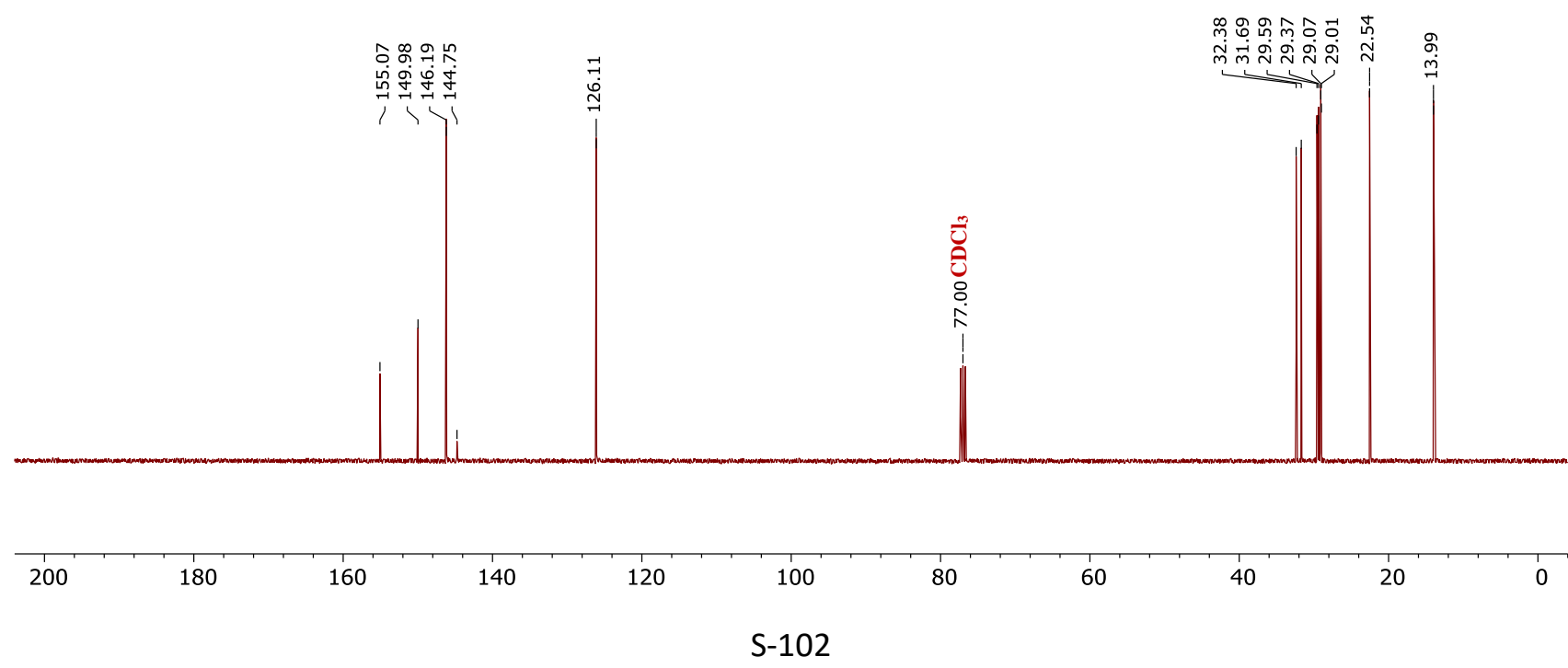


<smiles>CCCCCCCc1nc(Oc2c([18F])cc(Cl)cc2Cl)ccc1[N+](=O)[O-]</smiles>

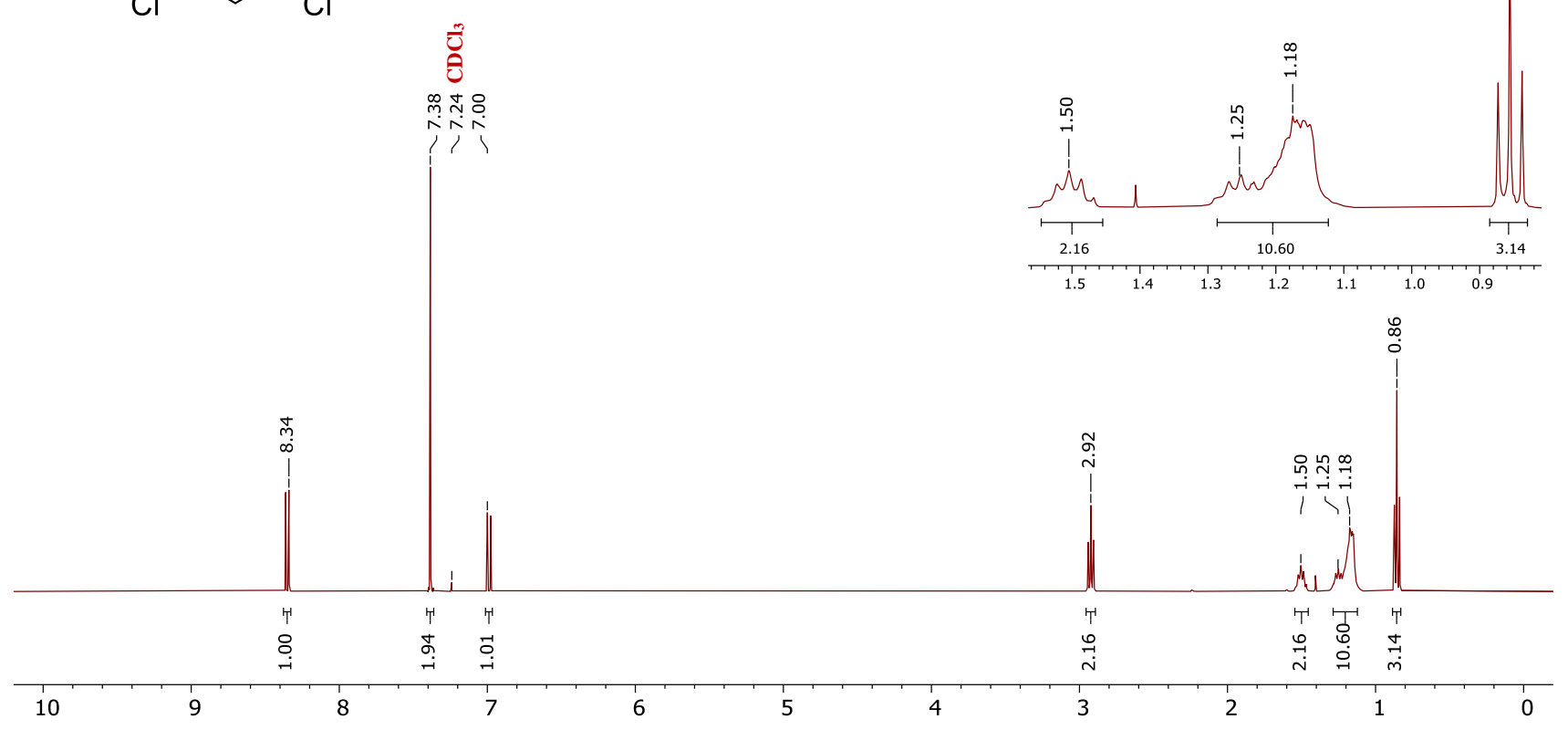

${ }^{13} \mathrm{C}$ NMR (100 MHz, $\left.\mathrm{CDCl}_{3}\right)$

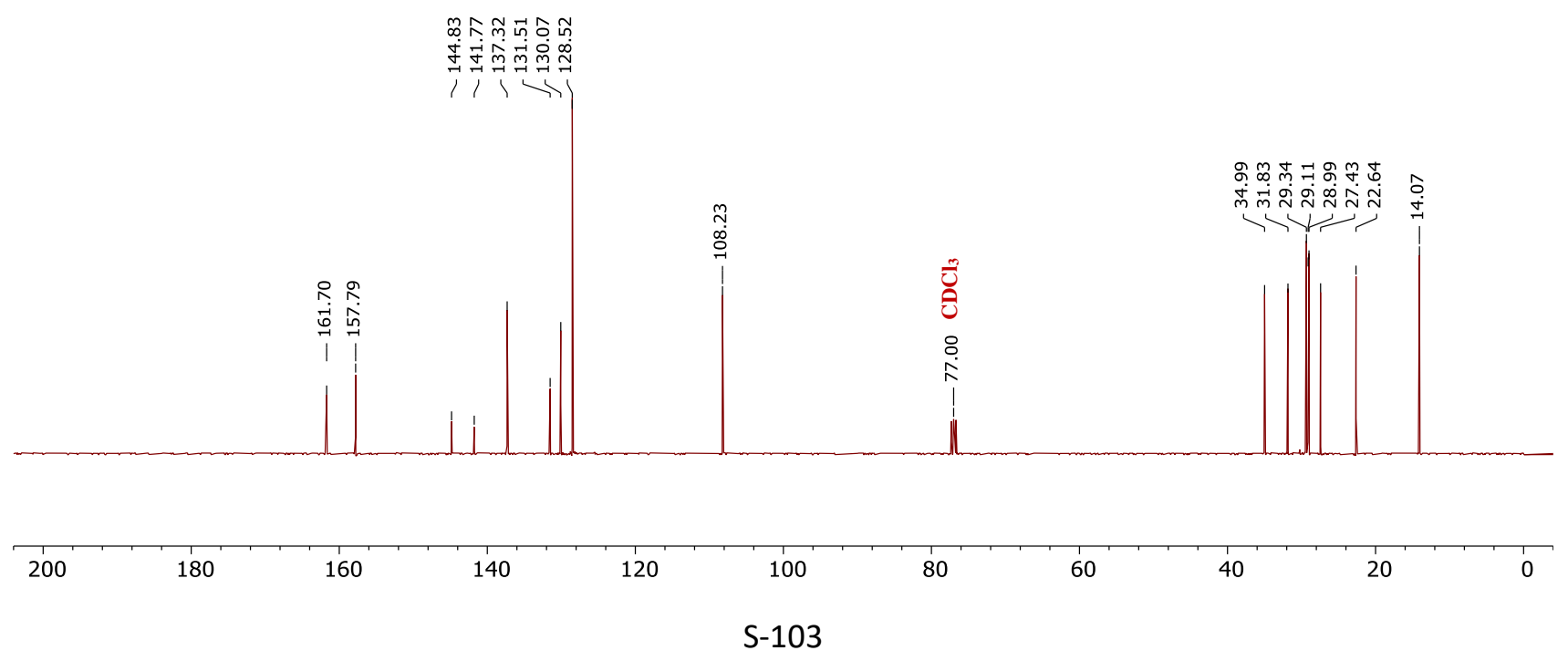


<smiles>CCCCCCCc1cc(Oc2c(Cl)cc(Cl)cc2Cl)ncc1[N+](=O)[O-]</smiles>

${ }^{1} \mathrm{H}$ NMR (400 MHz, $\mathrm{CDCl}_{3}$ )

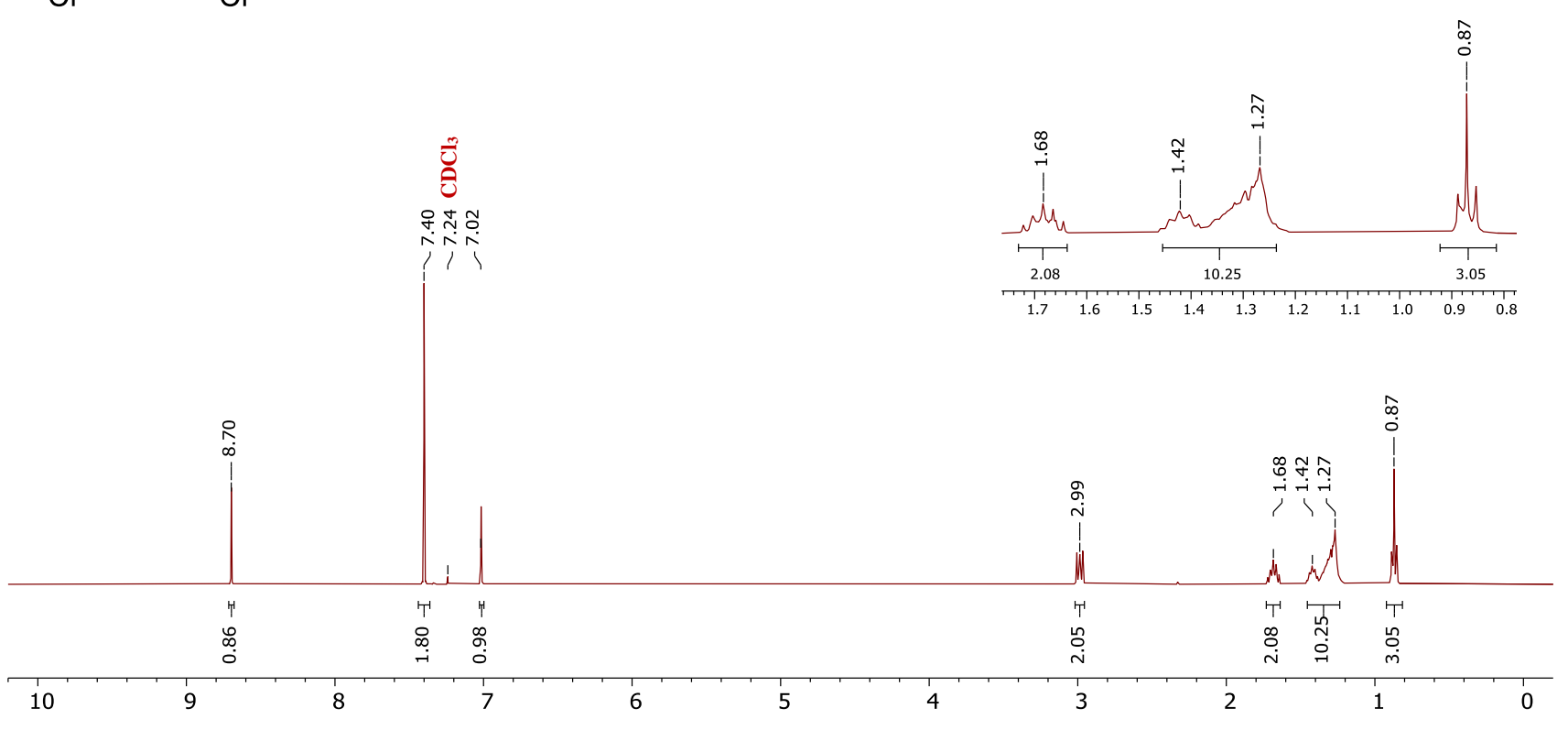

${ }^{13} \mathrm{C}$ NMR (100 MHz, $\left.\mathrm{CDCl}_{3}\right)$

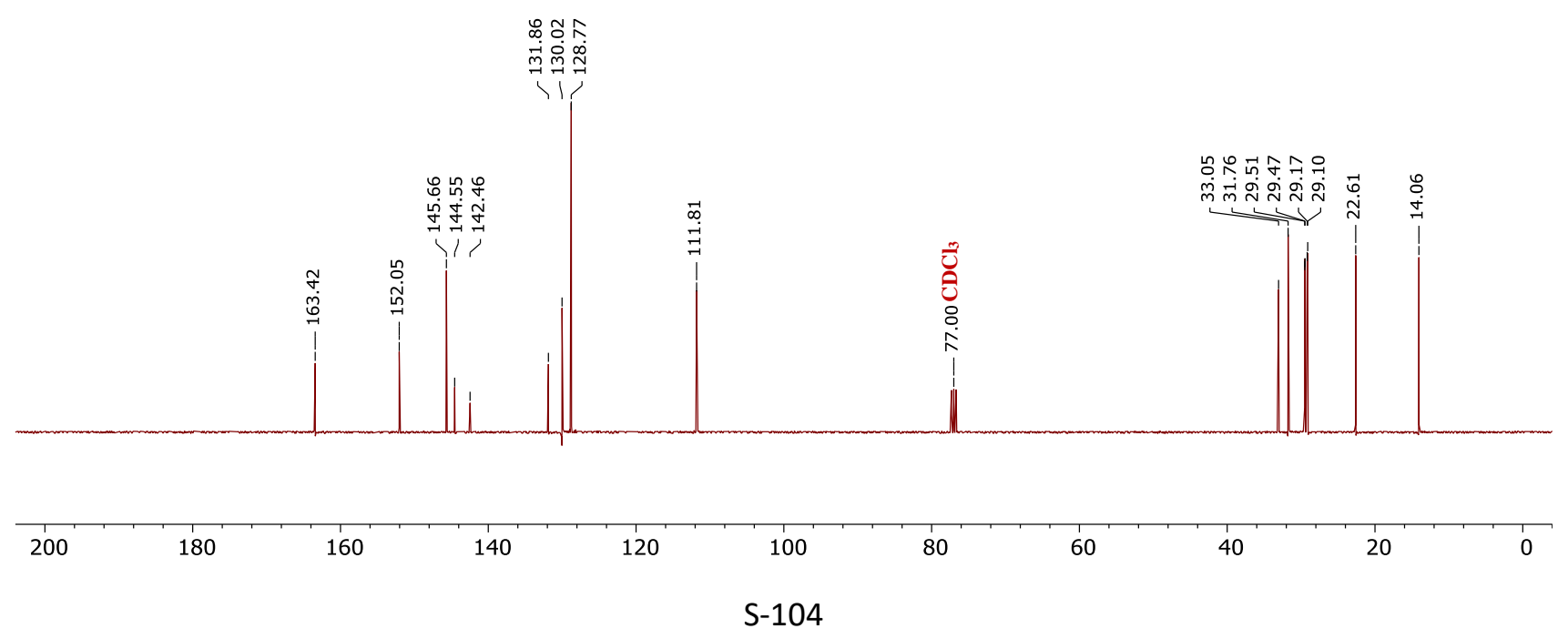




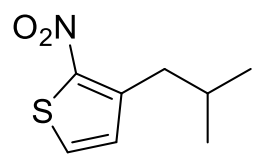

${ }^{1} \mathrm{H}$ NMR (400 MHz, $\left.\mathrm{CDCl}_{3}\right)$

4s

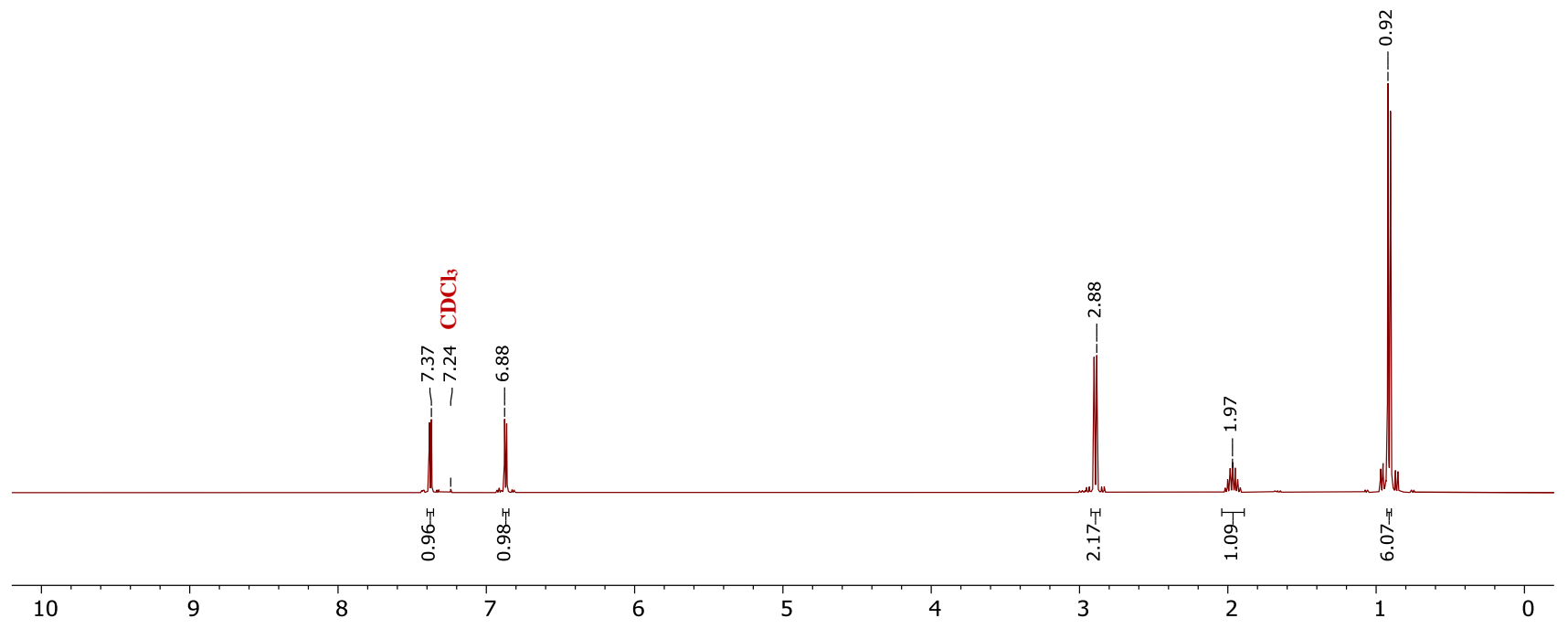

${ }^{13} \mathrm{C}$ NMR (100 MHz, $\left.\mathrm{CDCl}_{3}\right)$

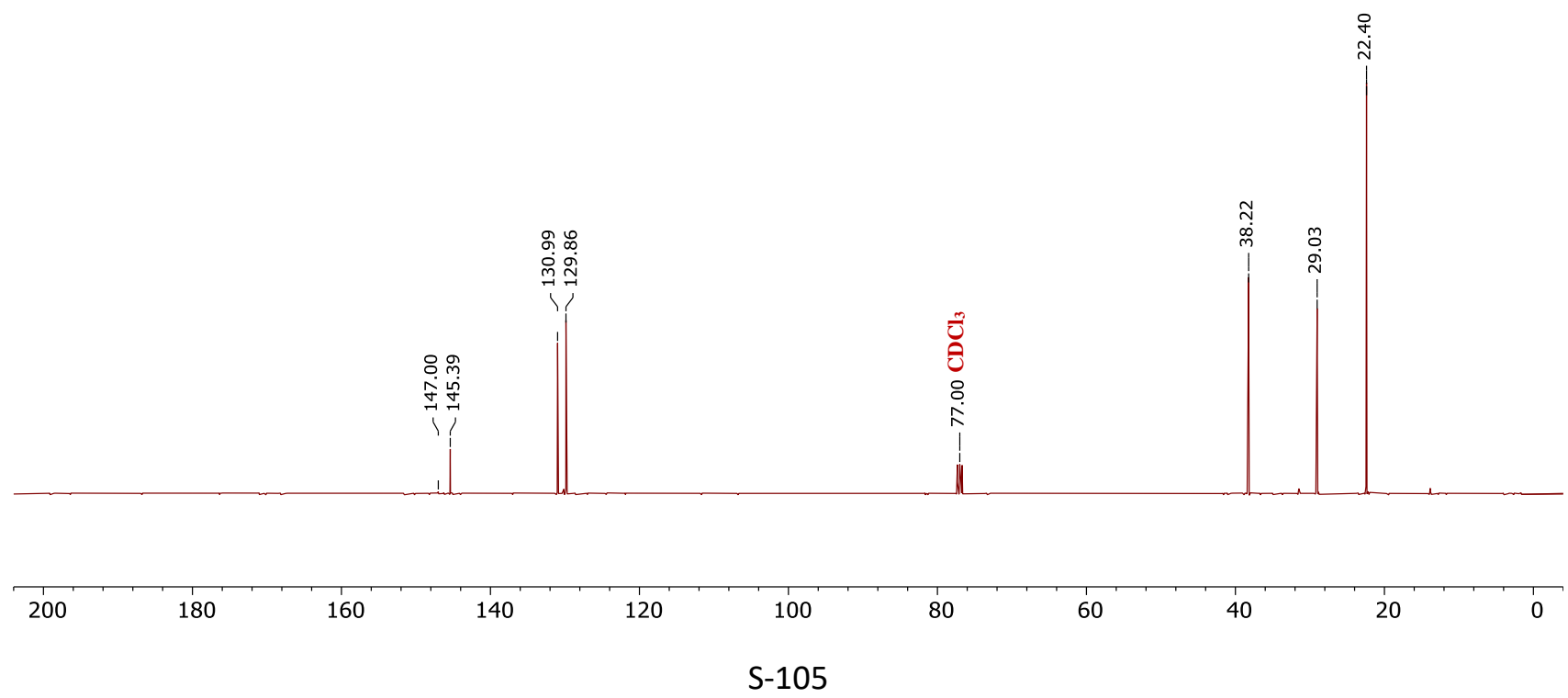




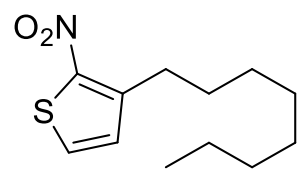

$4 t$

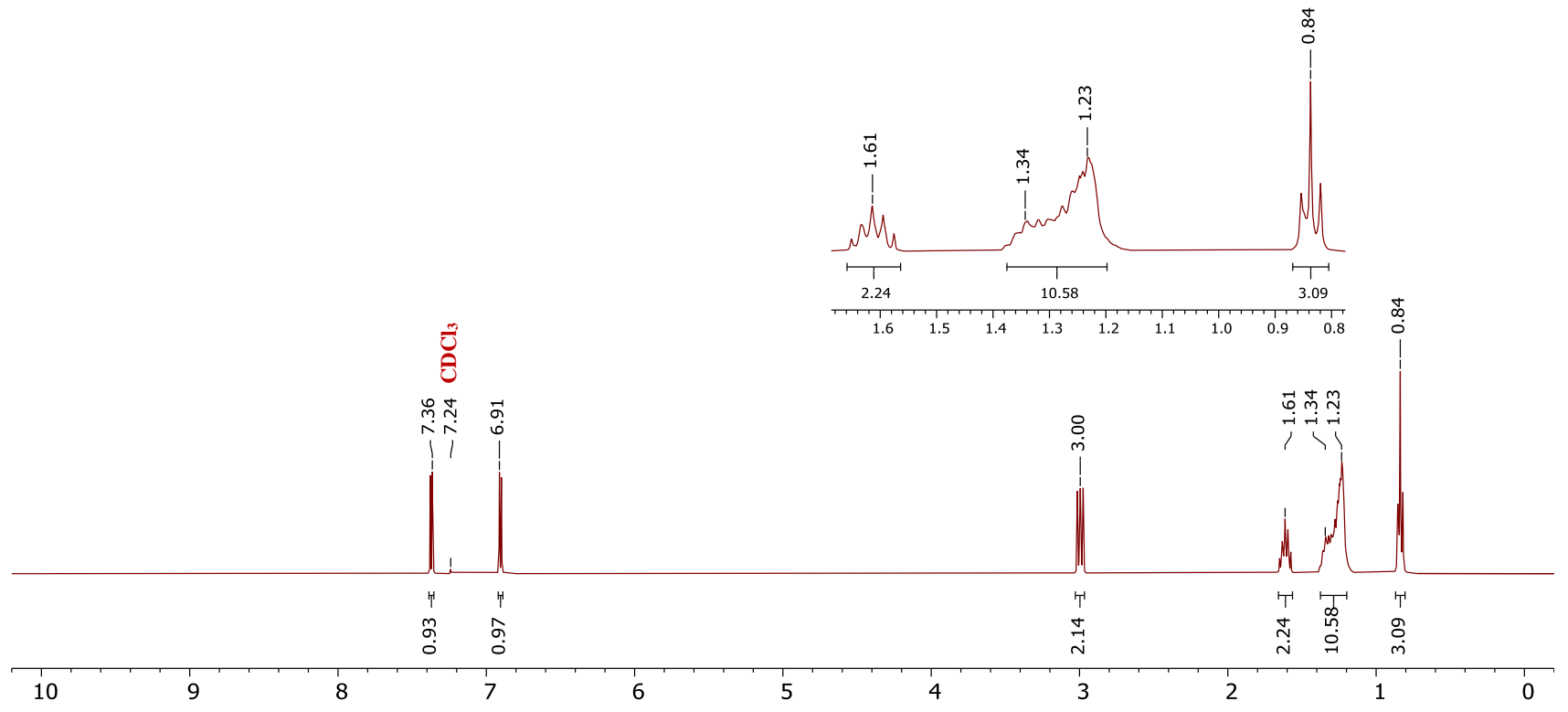

${ }^{13} \mathrm{C}$ NMR (100 MHz, $\left.\mathrm{CDCl}_{3}\right)$

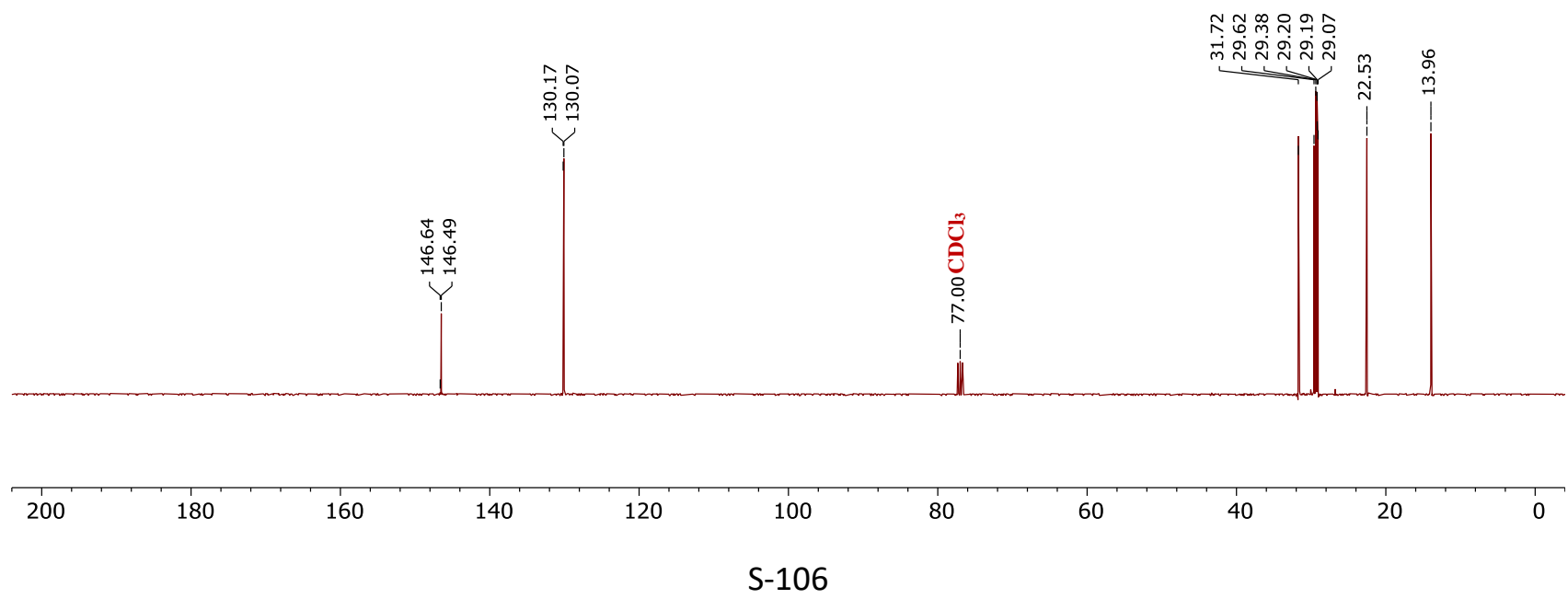


<smiles>Cc1c([N+](=O)[O-])c[n+]([Al])c2ccccc12</smiles>
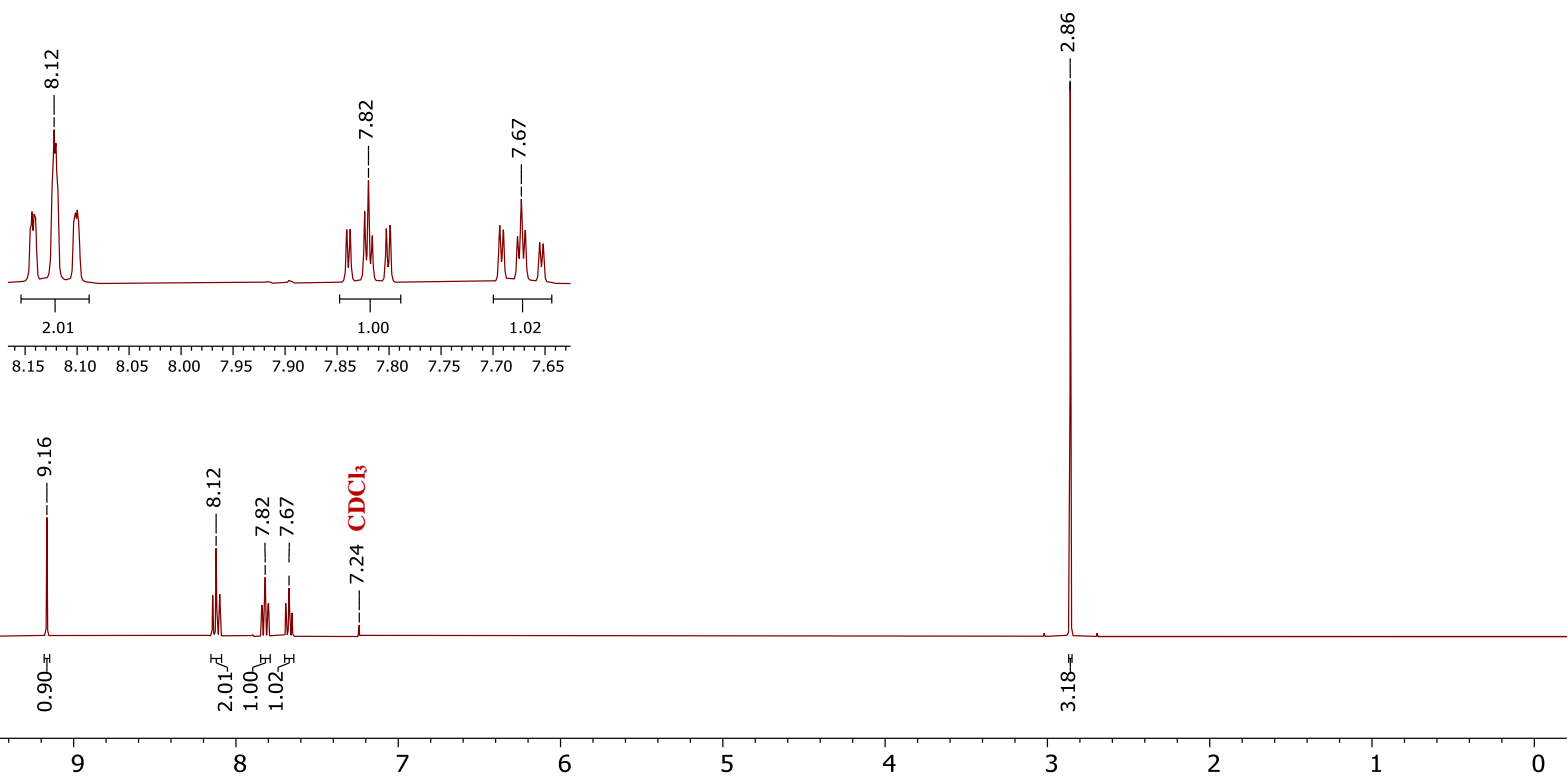

${ }^{13} \mathrm{C}$ NMR (100 MHz, $\left.\mathrm{CDCl}_{3}\right)$

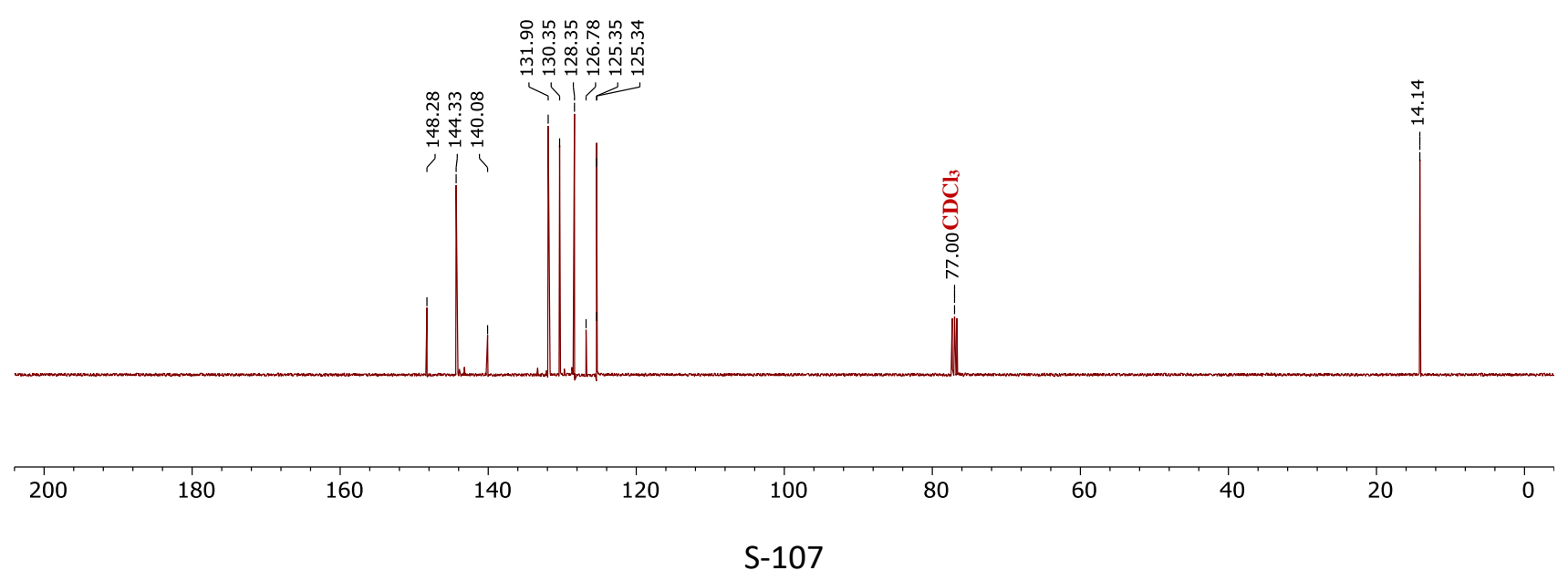


<smiles>CCCCCCCCc1cc(Cl)ccc1[N+](=O)[O-]</smiles>
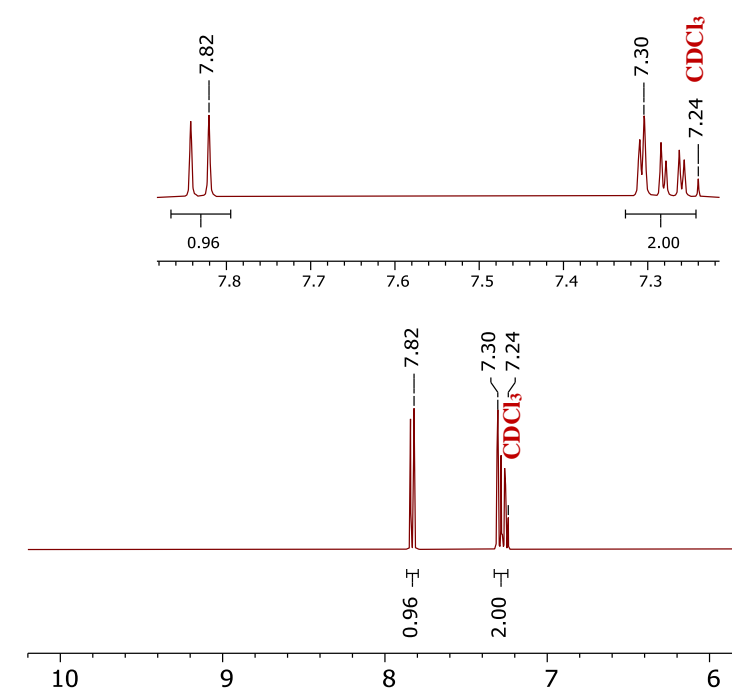

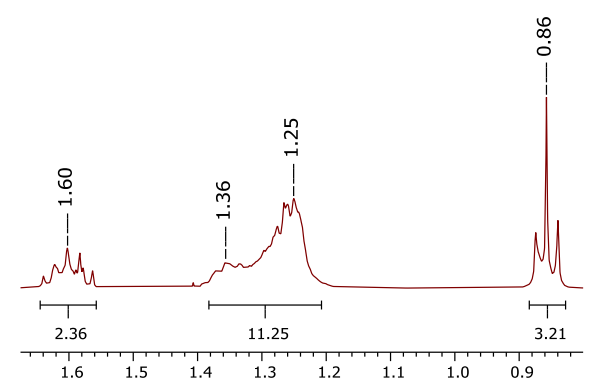

ํㅜㄹ

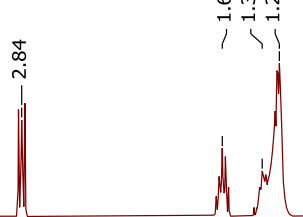

$\stackrel{\leftrightarrow}{\stackrel{T}{\sim}}$

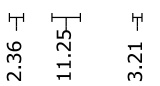

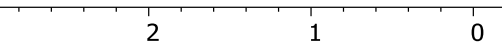

${ }^{13} \mathrm{C}$ NMR (100 MHz, $\left.\mathrm{CDCl}_{3}\right)$

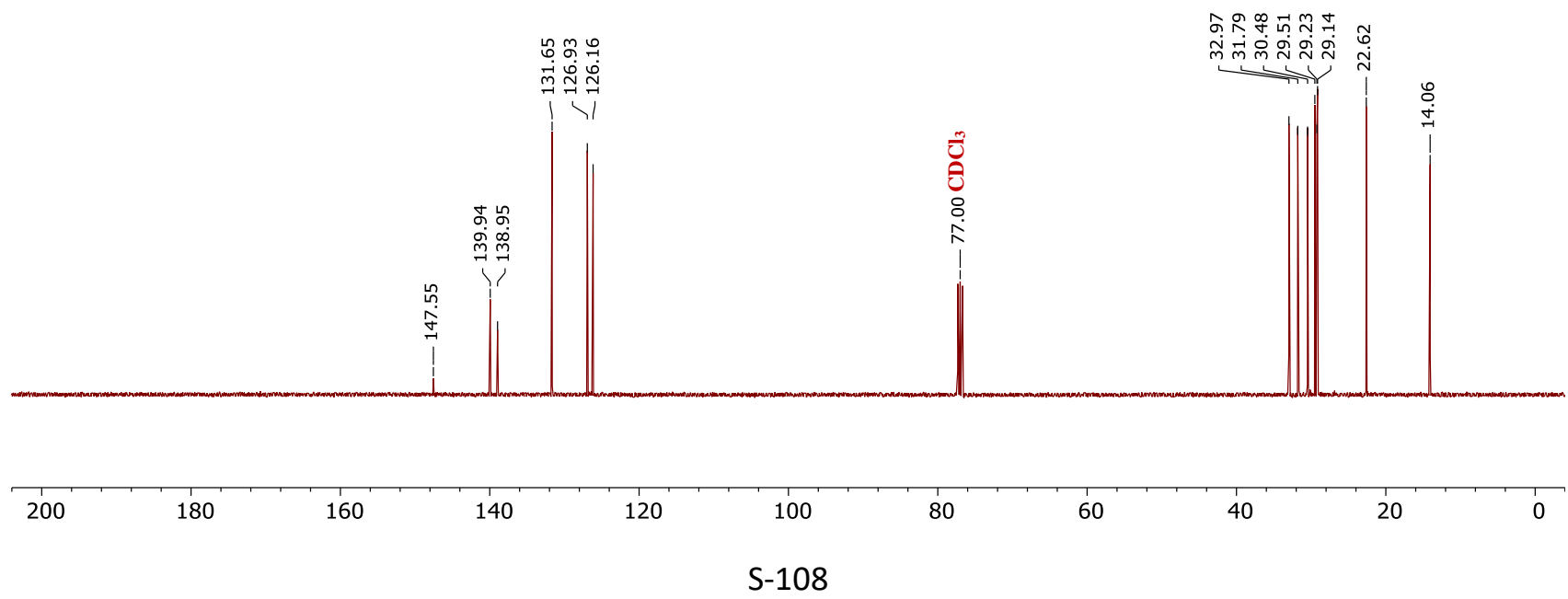


<smiles>CC(C)(SOc1ccccc1)[C@H]1C=CNC=C1[N+](=O)[O-]</smiles>

$5 a$

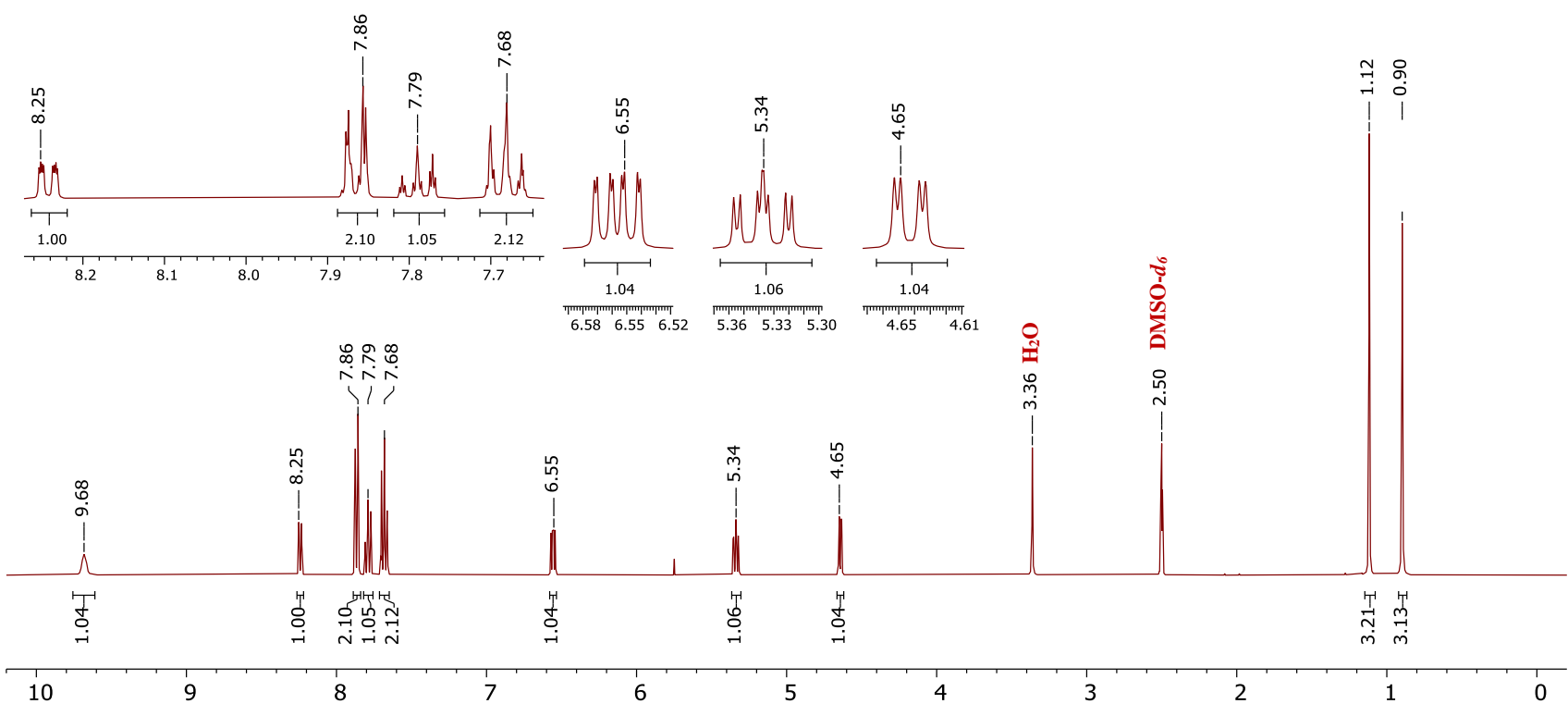

${ }^{13}$ C NMR (100 MHz, DMSO-d $\left.{ }_{6}\right)$

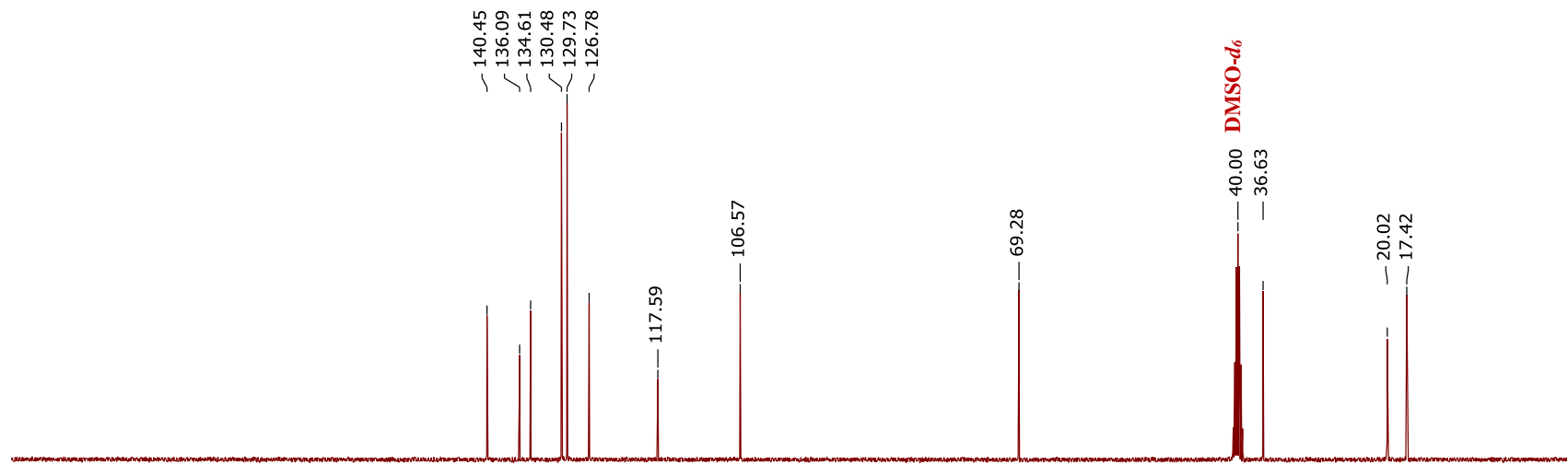


<smiles>CC(Oc1ccccc1)C1C([N+](=O)[O-])=CNc2ccccc21</smiles>

$\mathbf{5 b}$
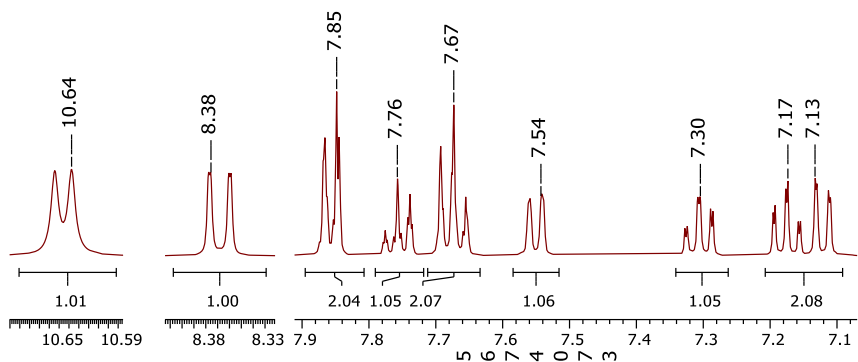

${ }^{1} \mathrm{H}$ NMR (400 MHz, DMSO- $\left.d_{6}\right)$

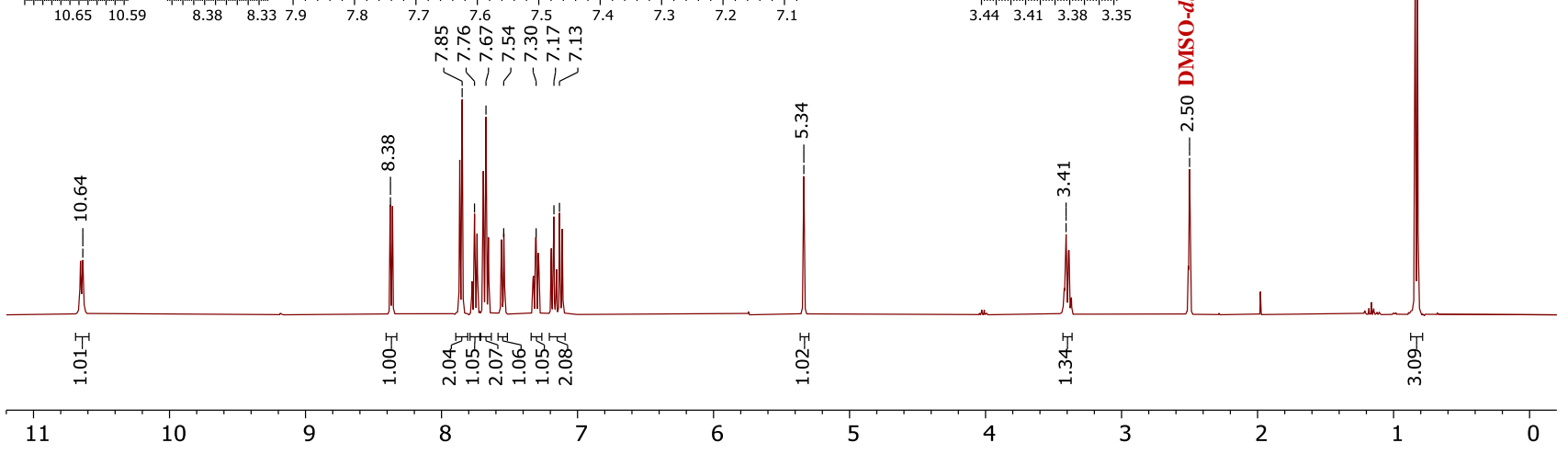

${ }^{13}$ C NMR (100 MHz, DMSO- $\left.d_{6}\right)$

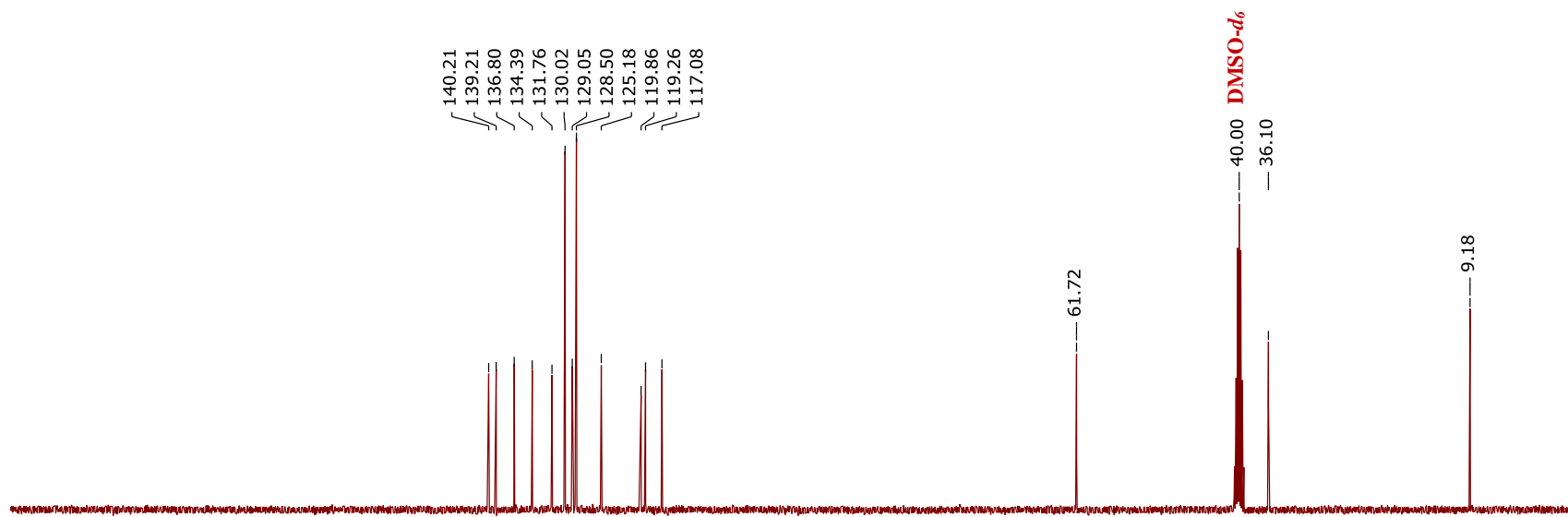


<smiles>CC(C)(OSc1ccccc1)[C@H]1C([N+](=O)[O-])=CNc2ccccc21</smiles>

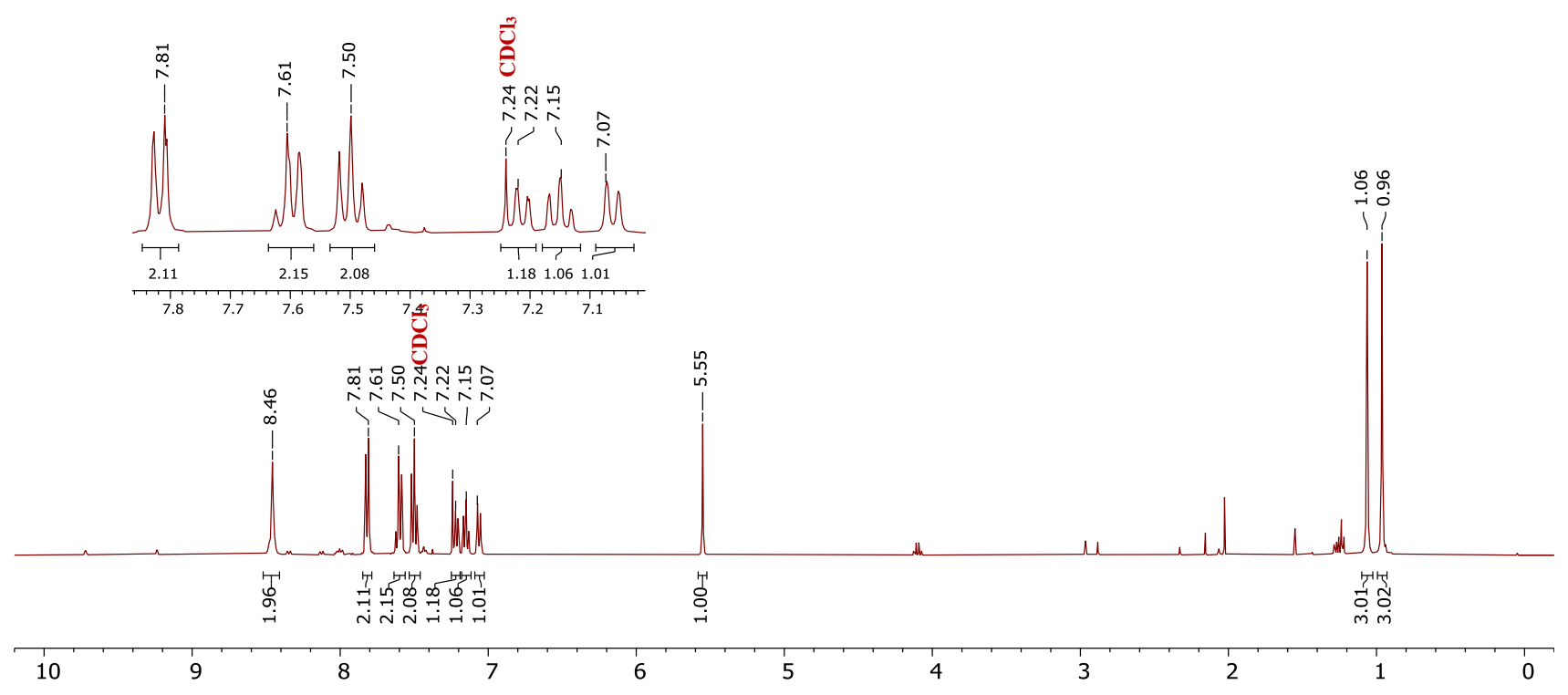

${ }^{13} \mathrm{C}$ NMR (100 MHz, $\left.\mathrm{CDCl}_{3}\right)$

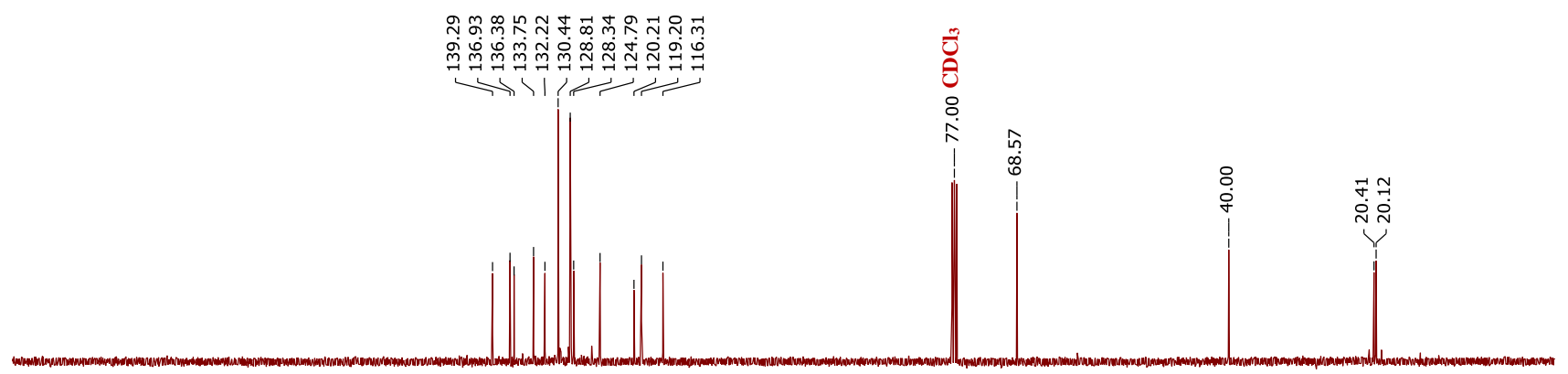


<smiles>CCCCCCC[C@H](c1cc(Cl)ccc1[N+](=O)[O-])[C@H](CCCCCC)c1cc(Cl)ccc1[N+](=O)[O-]</smiles>
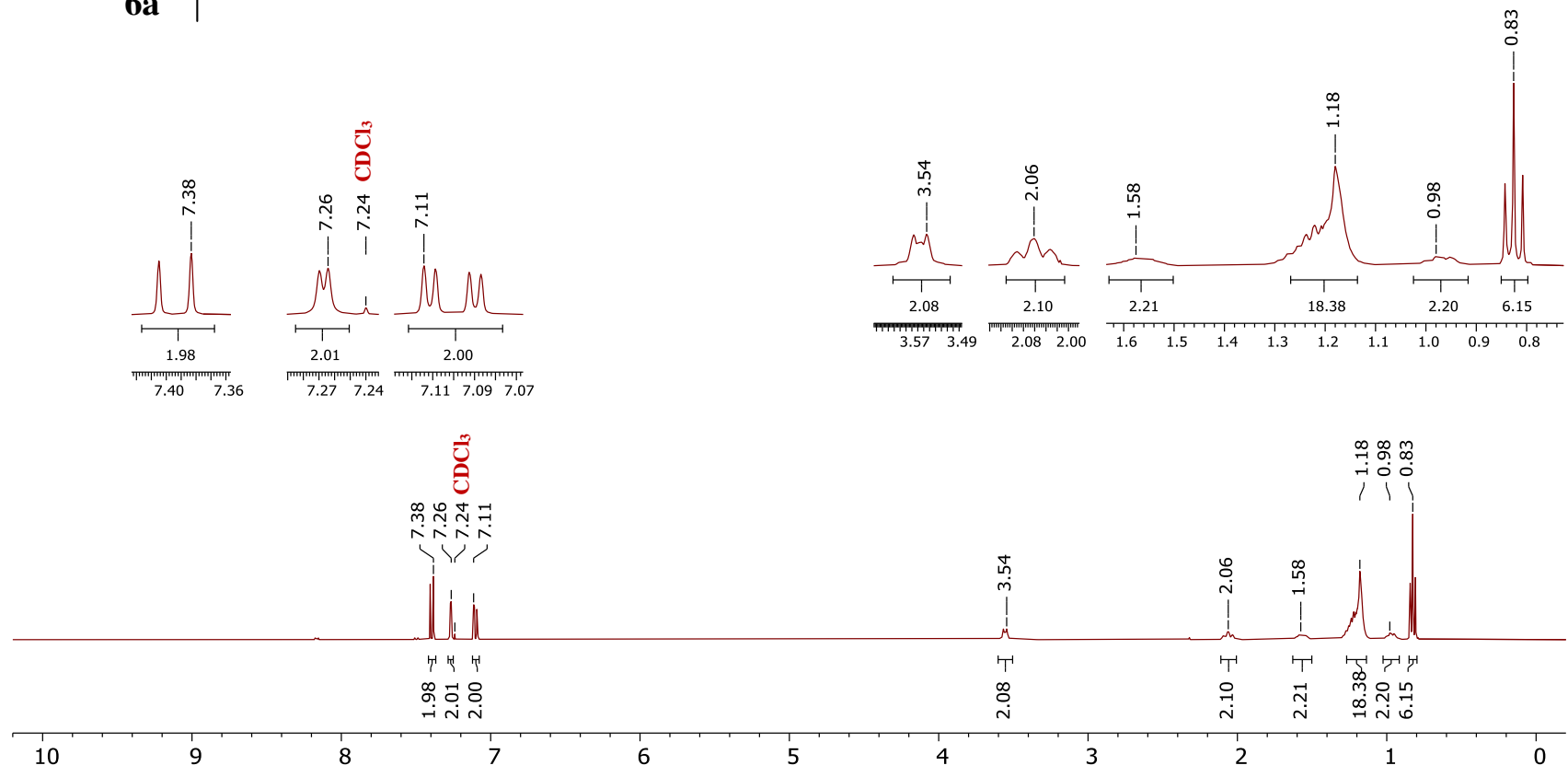

${ }^{13} \mathrm{C}$ NMR (100 MHz, $\left.\mathrm{CDCl}_{3}\right)$

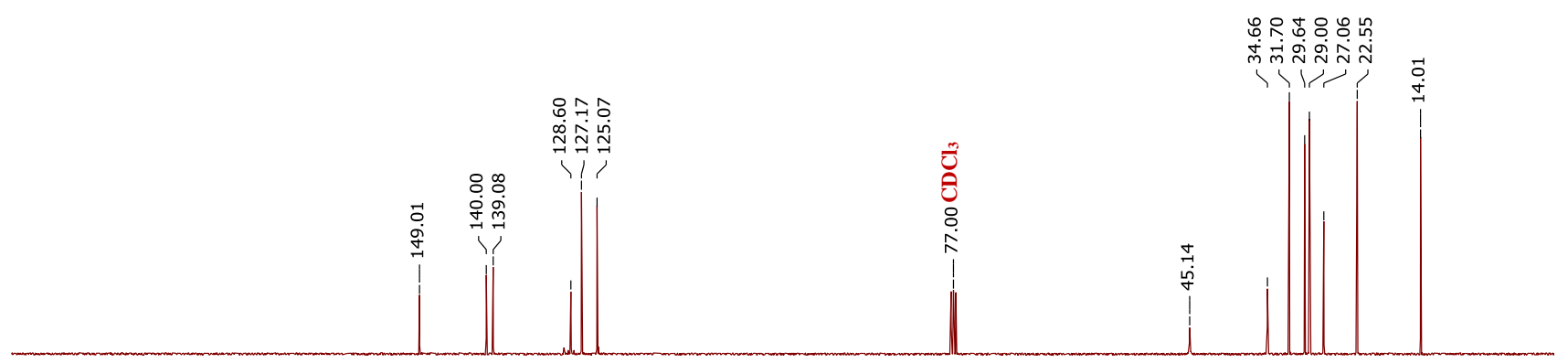


<smiles>CCCCCCCC(c1cc(Cl)ccc1[N+](=O)[O-])S(=O)(=O)CC(C)(C)C</smiles>

10

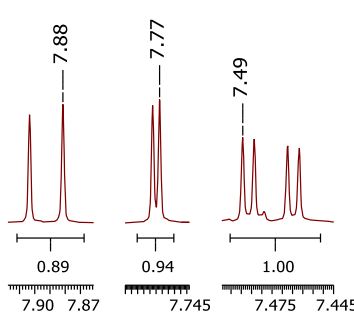

\section{${ }^{1} \mathrm{H}$ NMR (400 MHz, $\mathrm{CDCl}_{3}$ )}

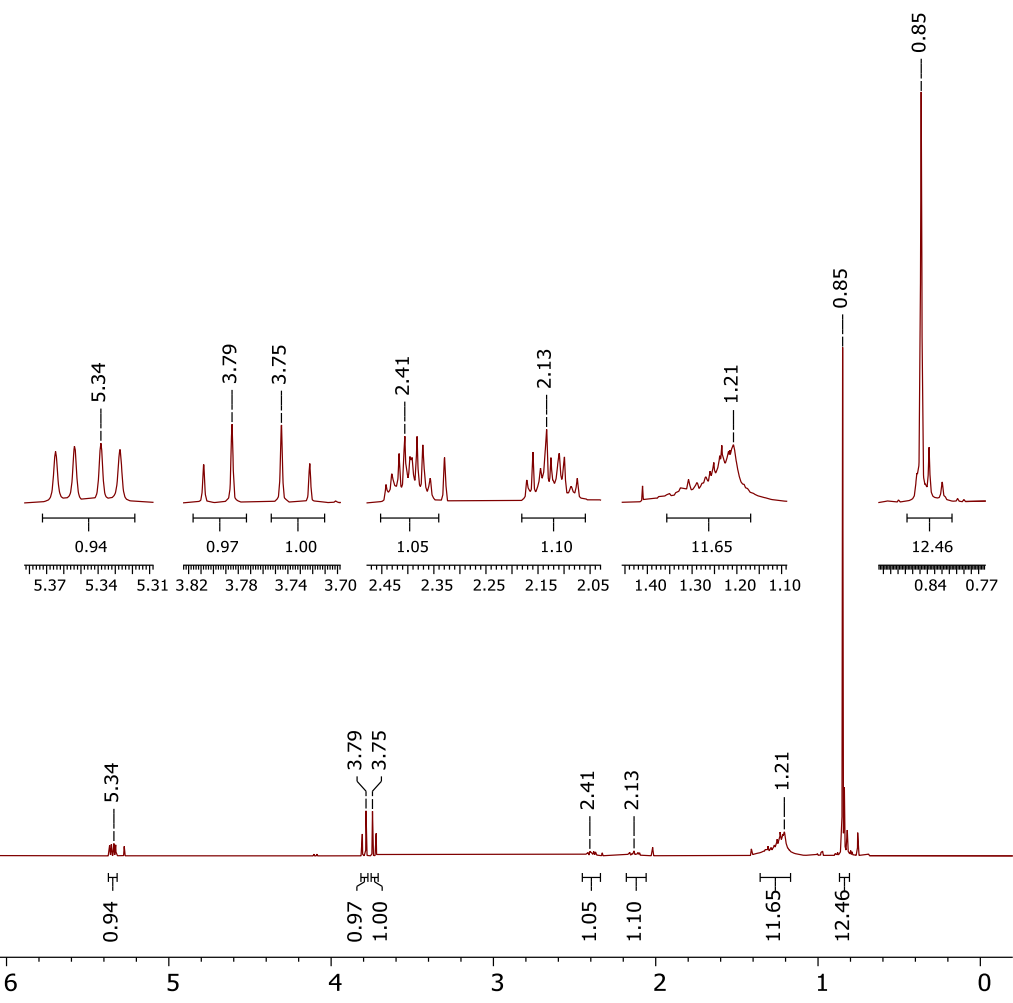

${ }^{13} \mathrm{C}$ NMR (100 MHz, $\left.\mathrm{CDCl}_{3}\right)$

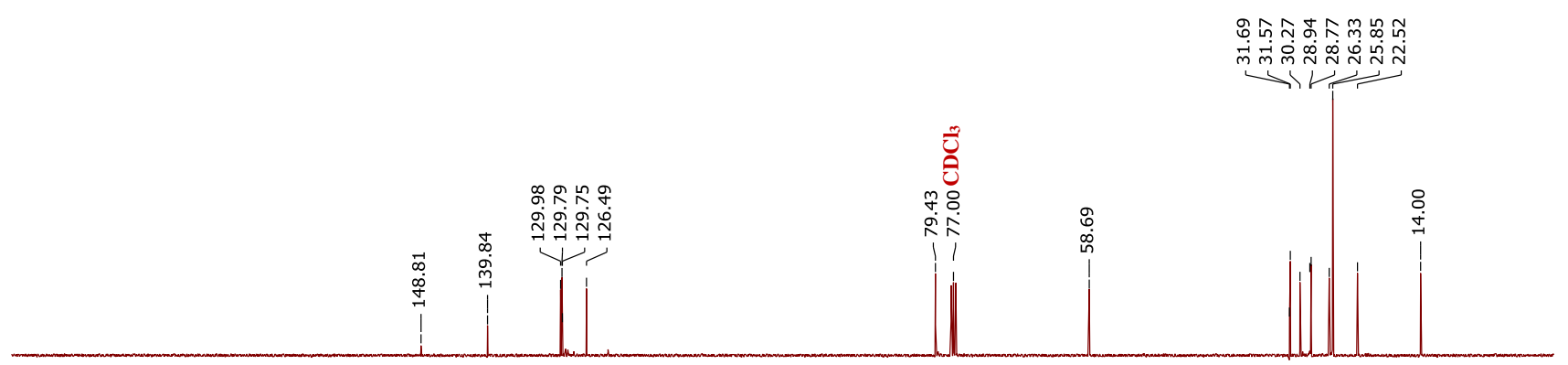


<smiles>CC(c1cc(Cl)ccc1[N+](=O)[O-])[C@H](C)c1cc(Cl)ccc1[N+](=O)[O-]</smiles>

6b

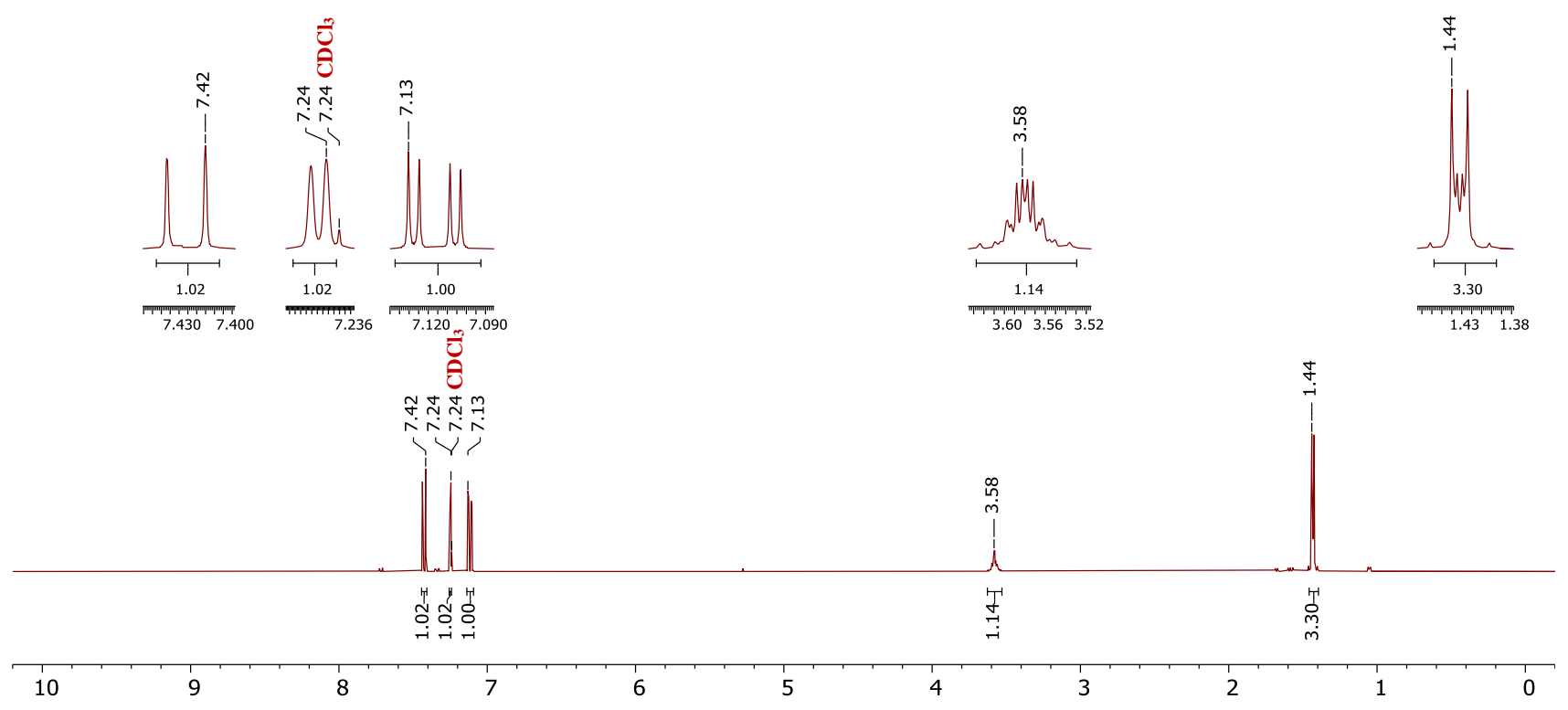

${ }^{13} \mathrm{C}$ NMR (100 MHz, $\left.\mathrm{CDCl}_{3}\right)$

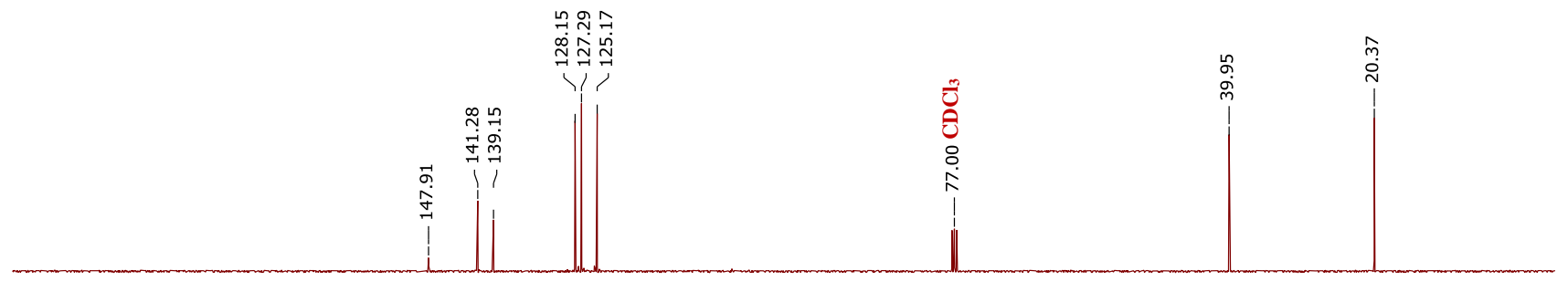


<smiles>O=[N+]([O-])c1cnccc1CCc1ccncc1[N+](=O)[O-]</smiles>
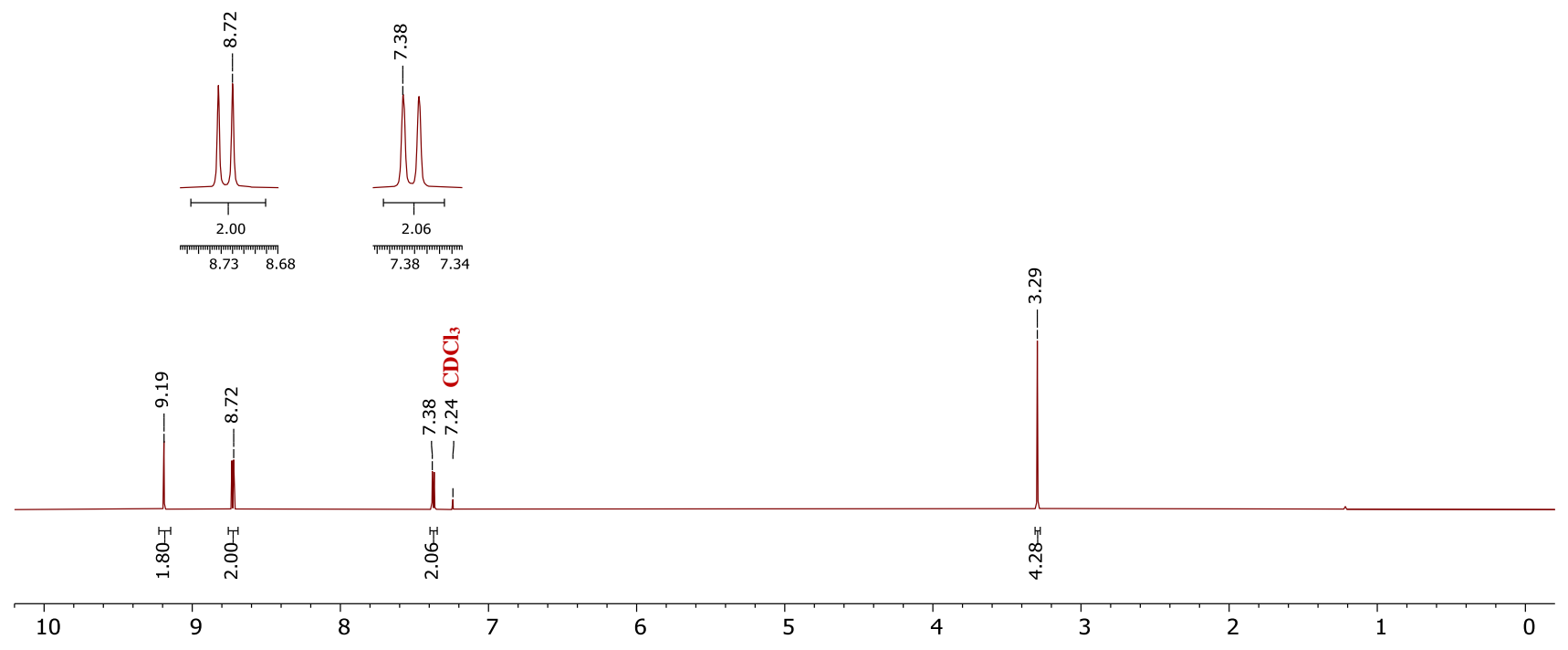

${ }^{13} \mathrm{C}$ NMR (100 $\left.\mathrm{MHz}, \mathrm{CDCl}_{3}\right)$

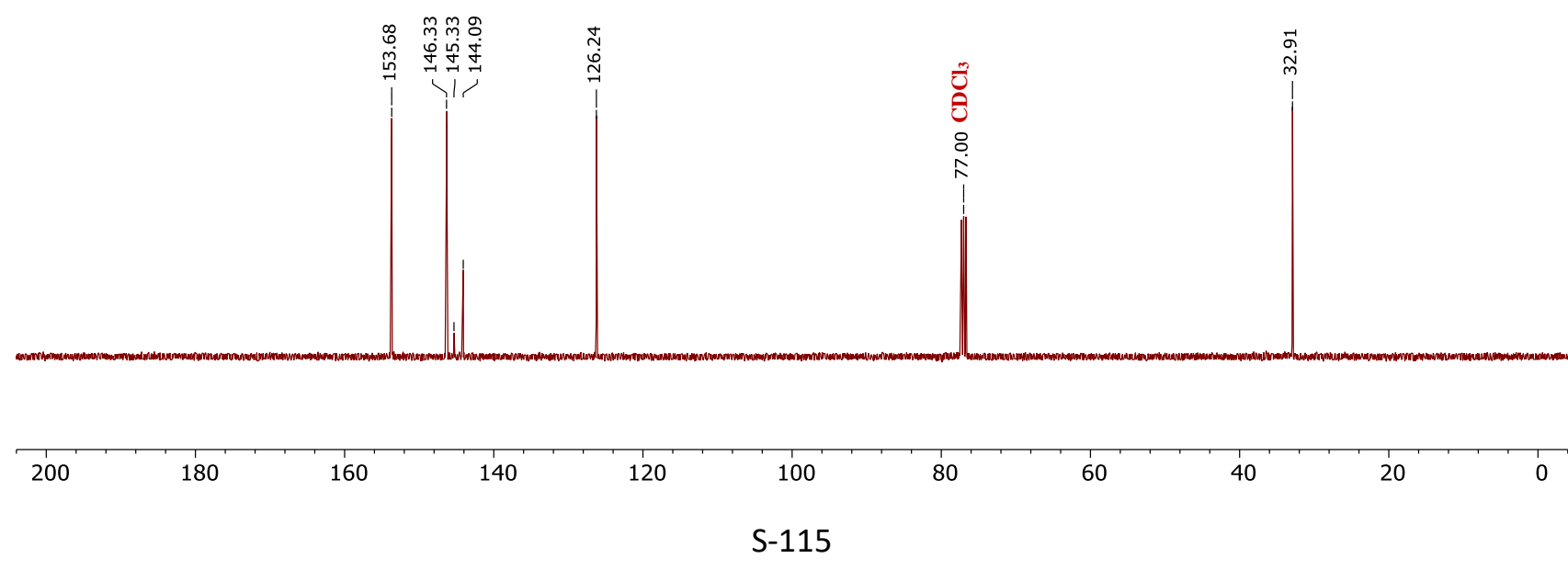


<smiles>CCCCCCC[C@H](c1ccncc1[N+](=O)[O-])[C@H](CCCCCC)c1ccncc1[N+](=O)[O-]</smiles>
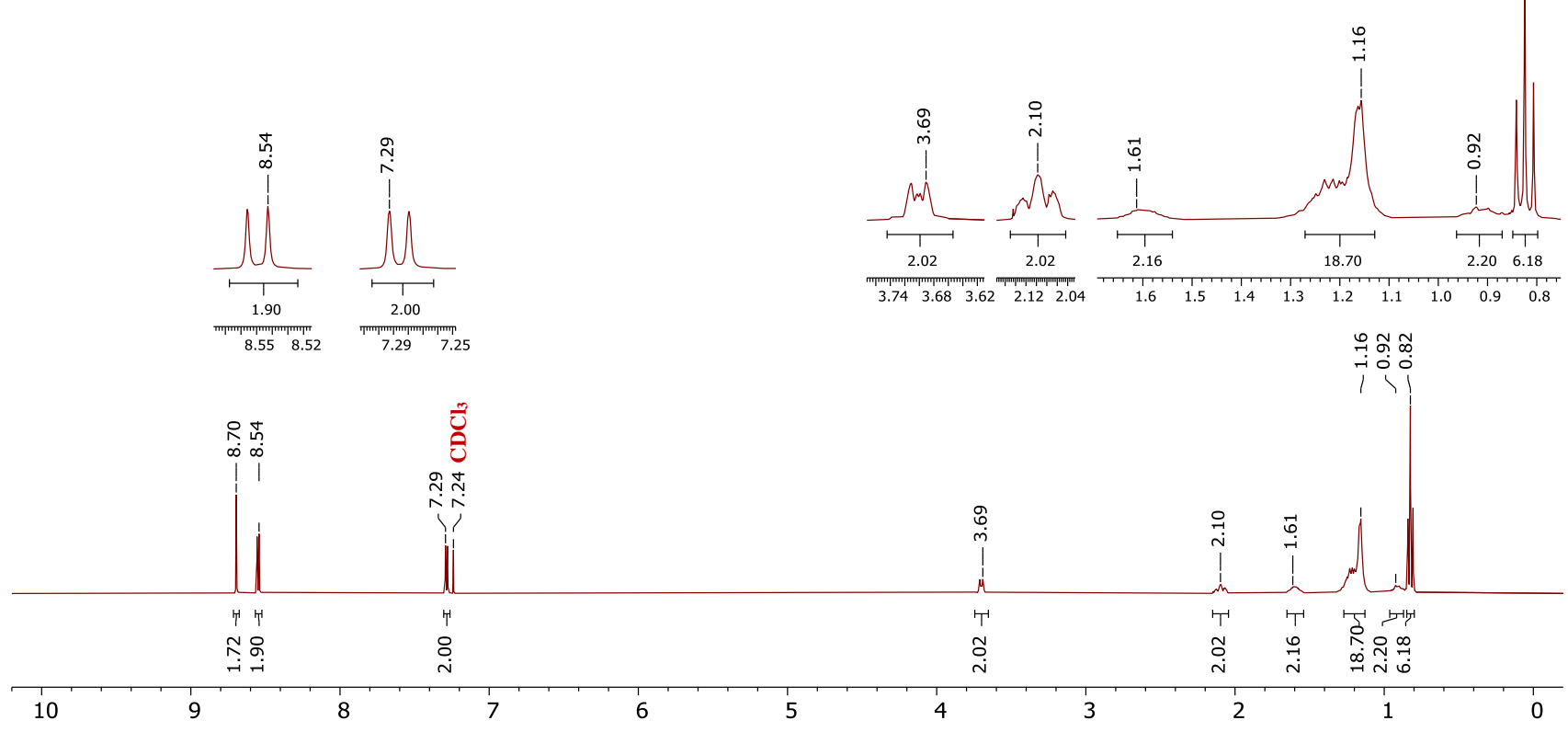

${ }^{13} \mathrm{C}$ NMR (100 MHz, $\left.\mathrm{CDCl}_{3}\right)$

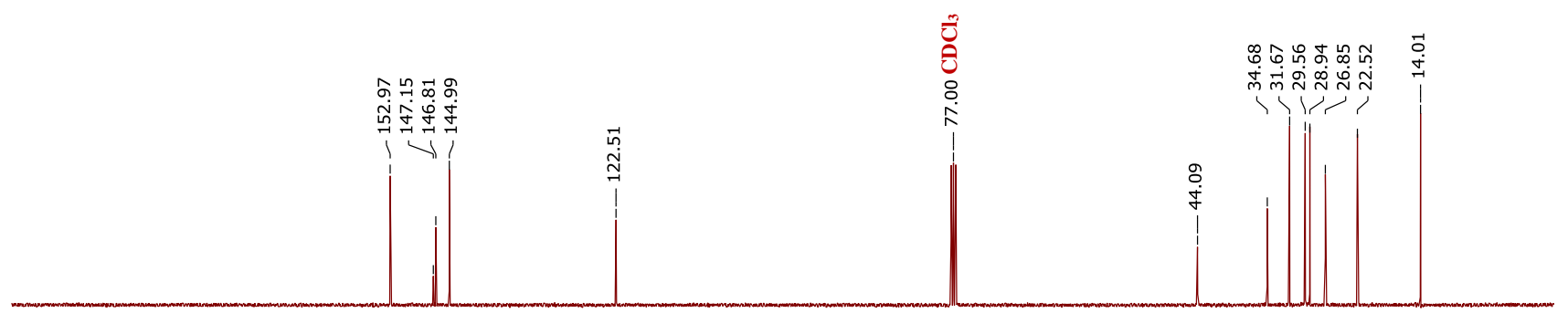


<smiles>CC1(C)CCCC(C)(C)N1OC(c1ccc([N+](=O)[O-])cn1)C1CC1</smiles>
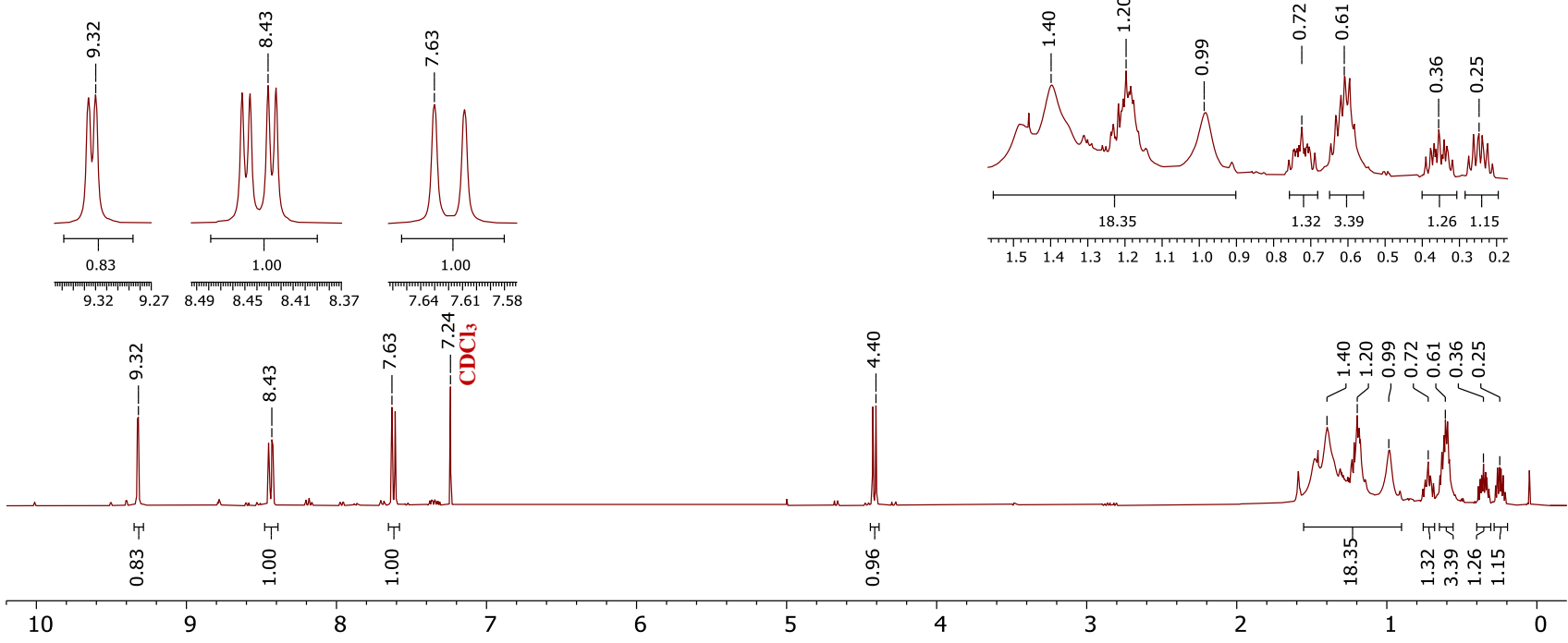

${ }^{13} \mathrm{C}$ NMR (100 MHz, $\left.\mathrm{CDCl}_{3}\right)$

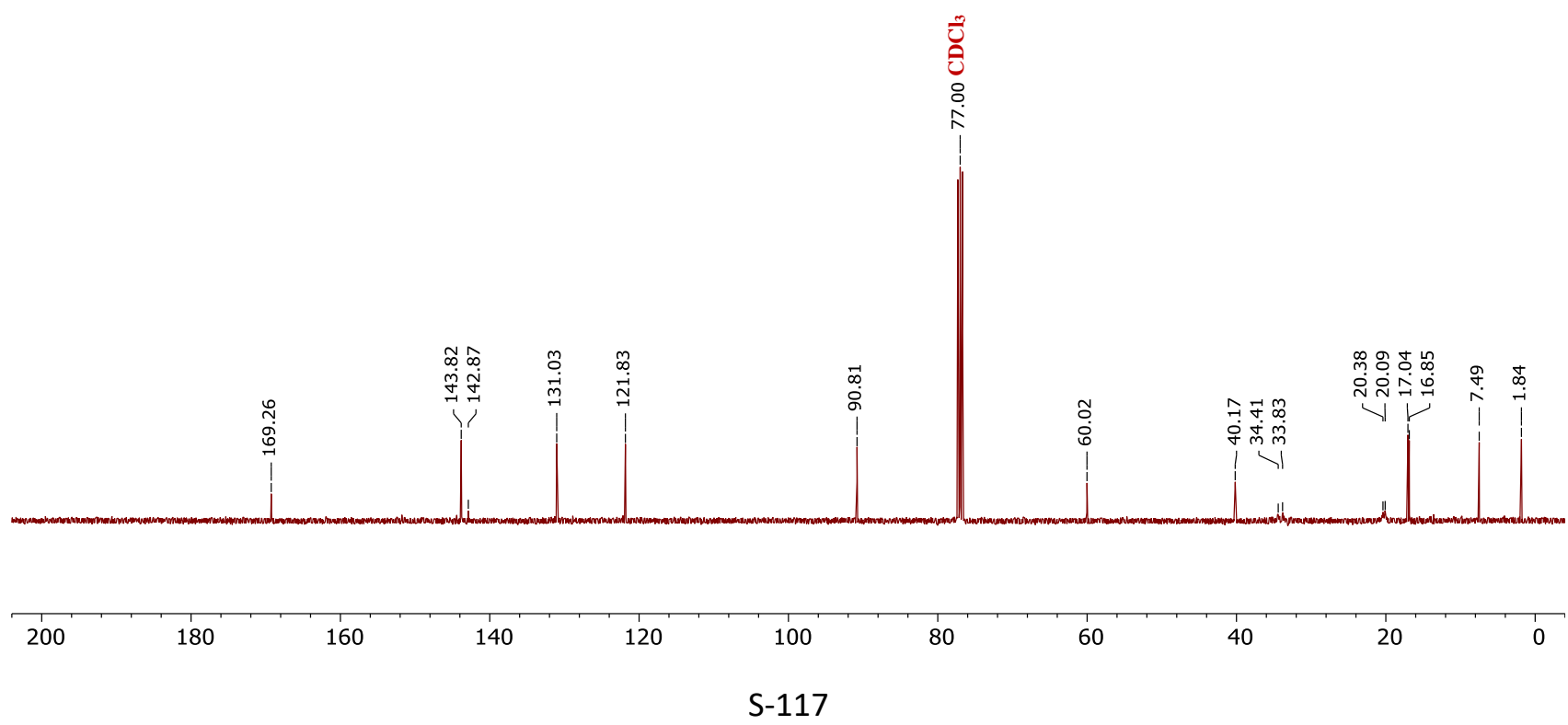


<smiles>CC(C)C(F)c1ccncc1[N+](=O)[O-]</smiles>
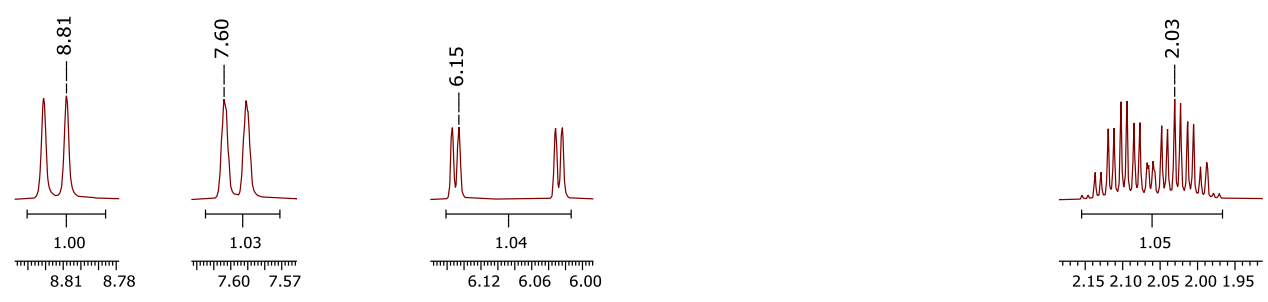

$\stackrel{7}{7}$

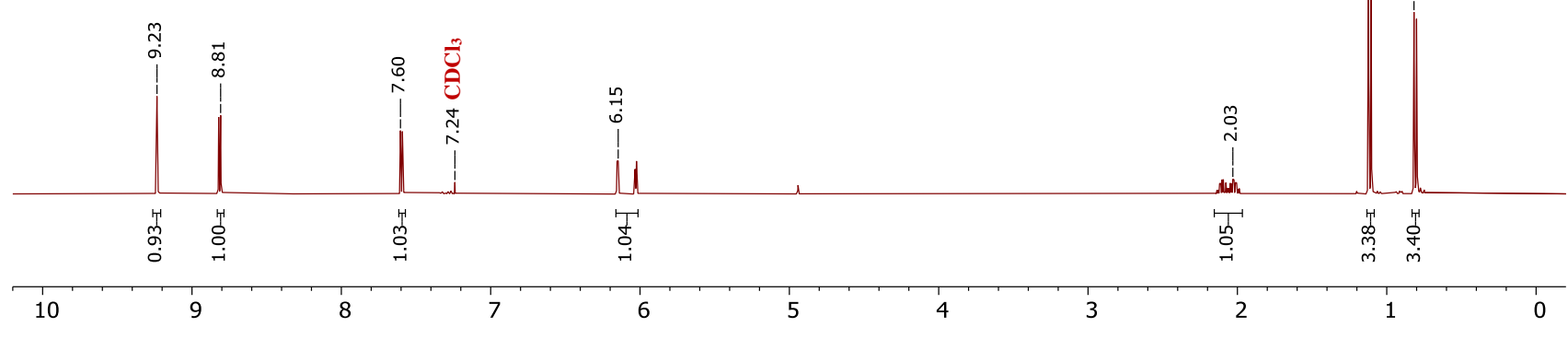

${ }^{13} \mathrm{C}$ NMR (100 MHz, $\left.\mathrm{CDCl}_{3}\right)$
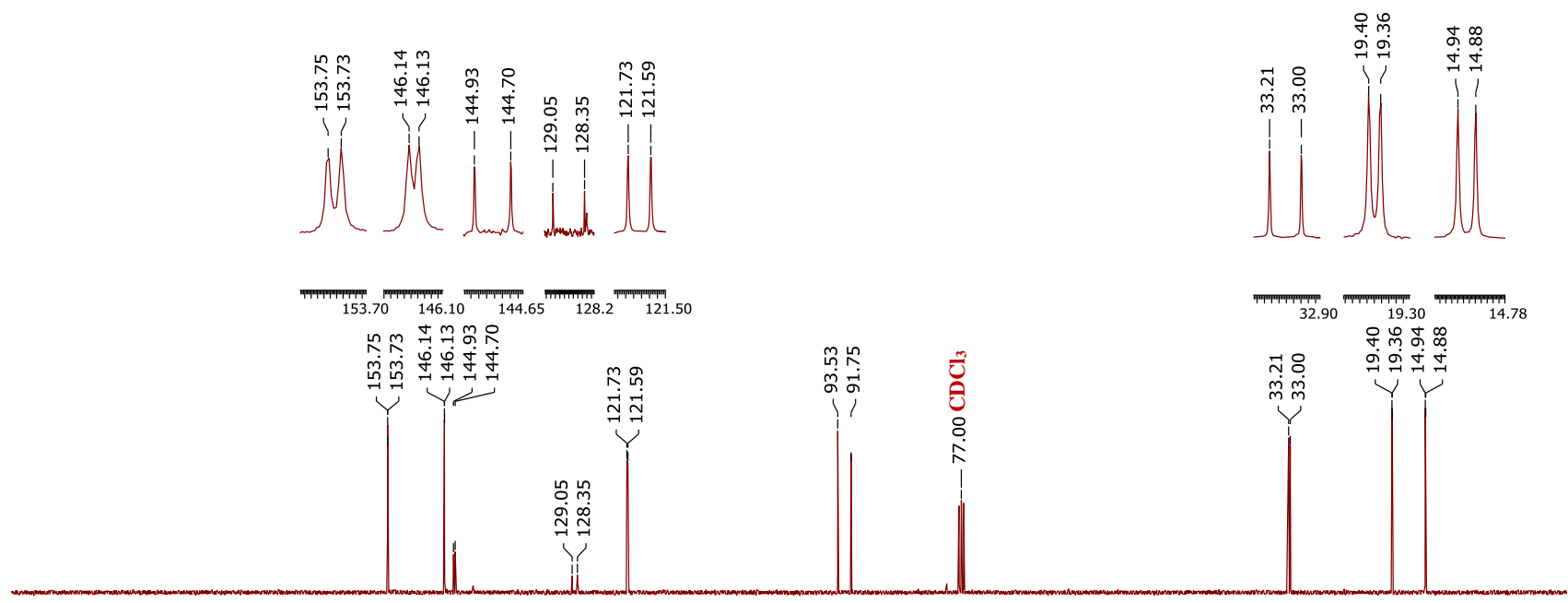
${ }^{19}$ F NMR (376 MHz, $\left.\mathrm{CDCl}_{3}\right)$
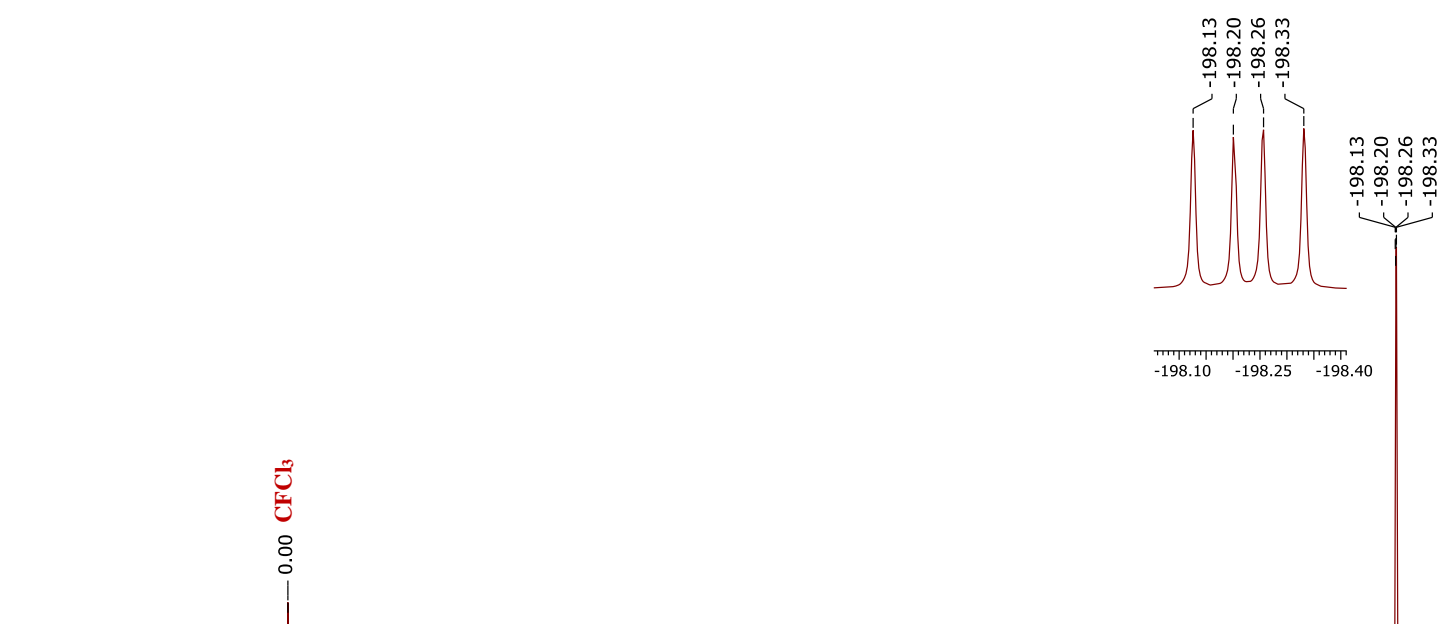

己ृ

$\begin{array}{lll}-198.10 & -198.25 & -198.40\end{array}$

0

$-50$

$-100$

$-150$

$-200$

-2 ! 
<smiles></smiles>

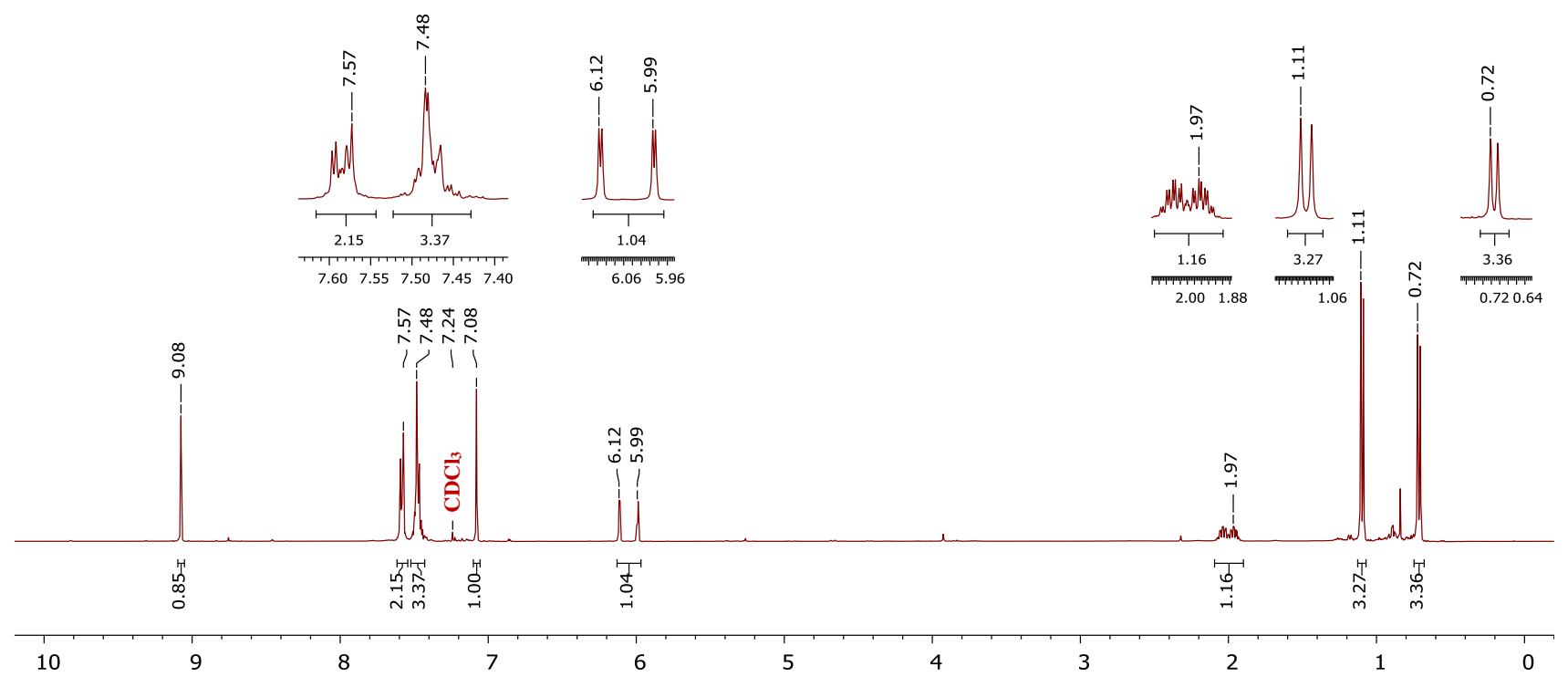

${ }^{13} \mathrm{C}$ NMR (100 MHz, $\left.\mathrm{CDCl}_{3}\right)$

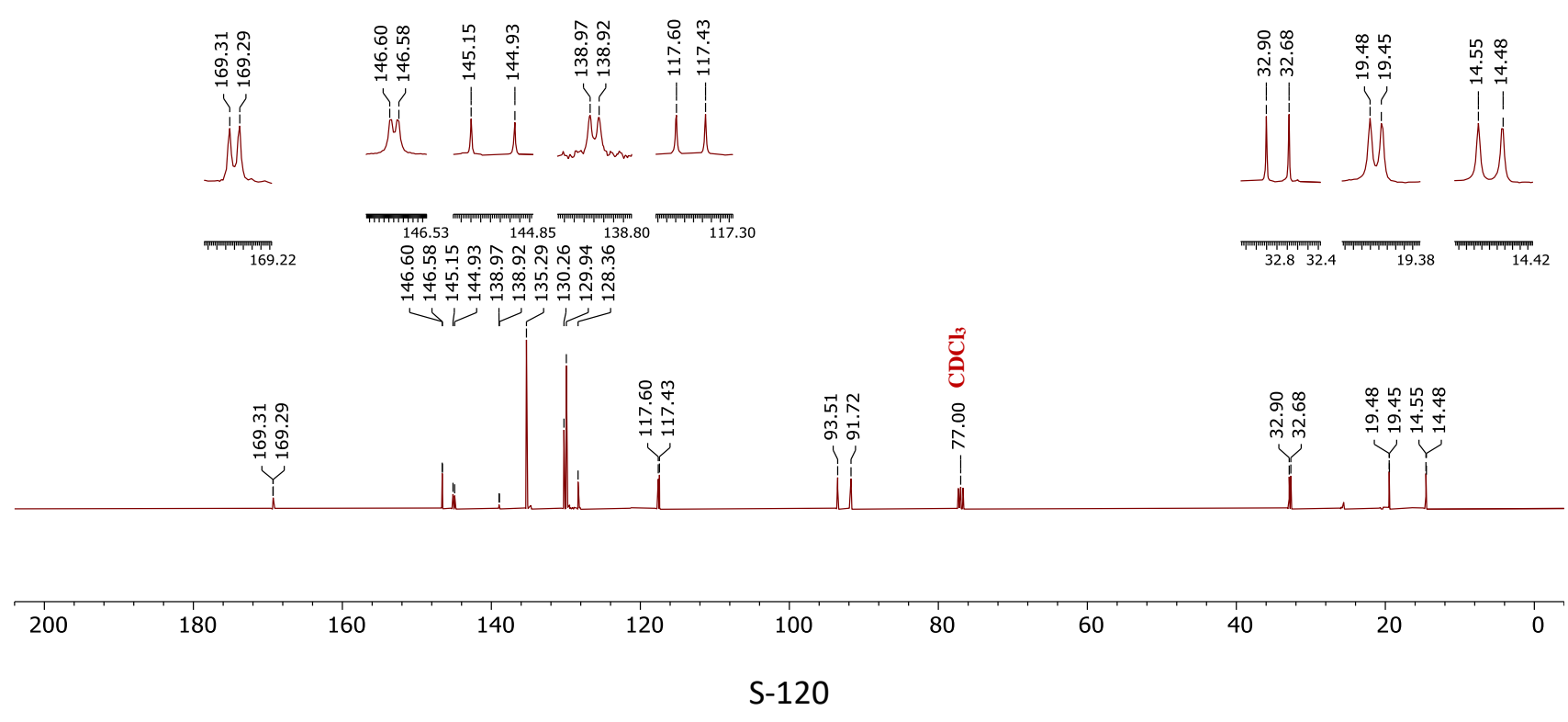


${ }^{19}$ F NMR (376 MHz, $\left.\mathrm{CDCl}_{3}\right)$

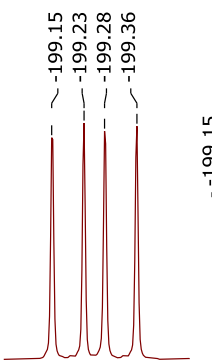

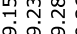

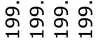

政

$-199.15-199.30-199.45$

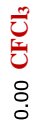

50

$-50$

$-100$

$-150$

$-200$

-2 ! 
<smiles>CC(C)(C)C(F)c1ccncc1[N+](=O)[O-]</smiles>
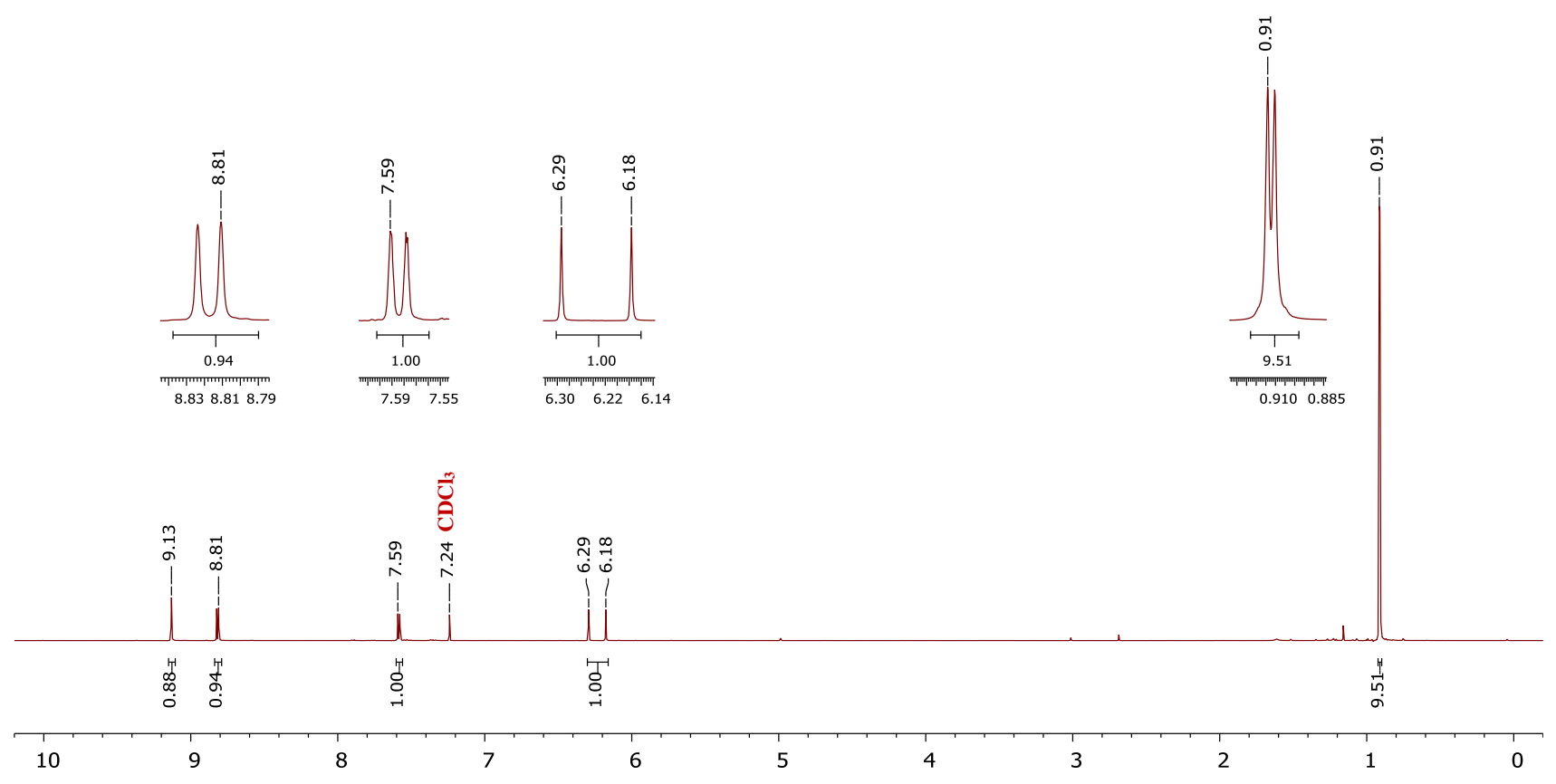

${ }^{13} \mathrm{C}$ NMR (100 MHz, $\left.\mathrm{CDCl}_{3}\right)$

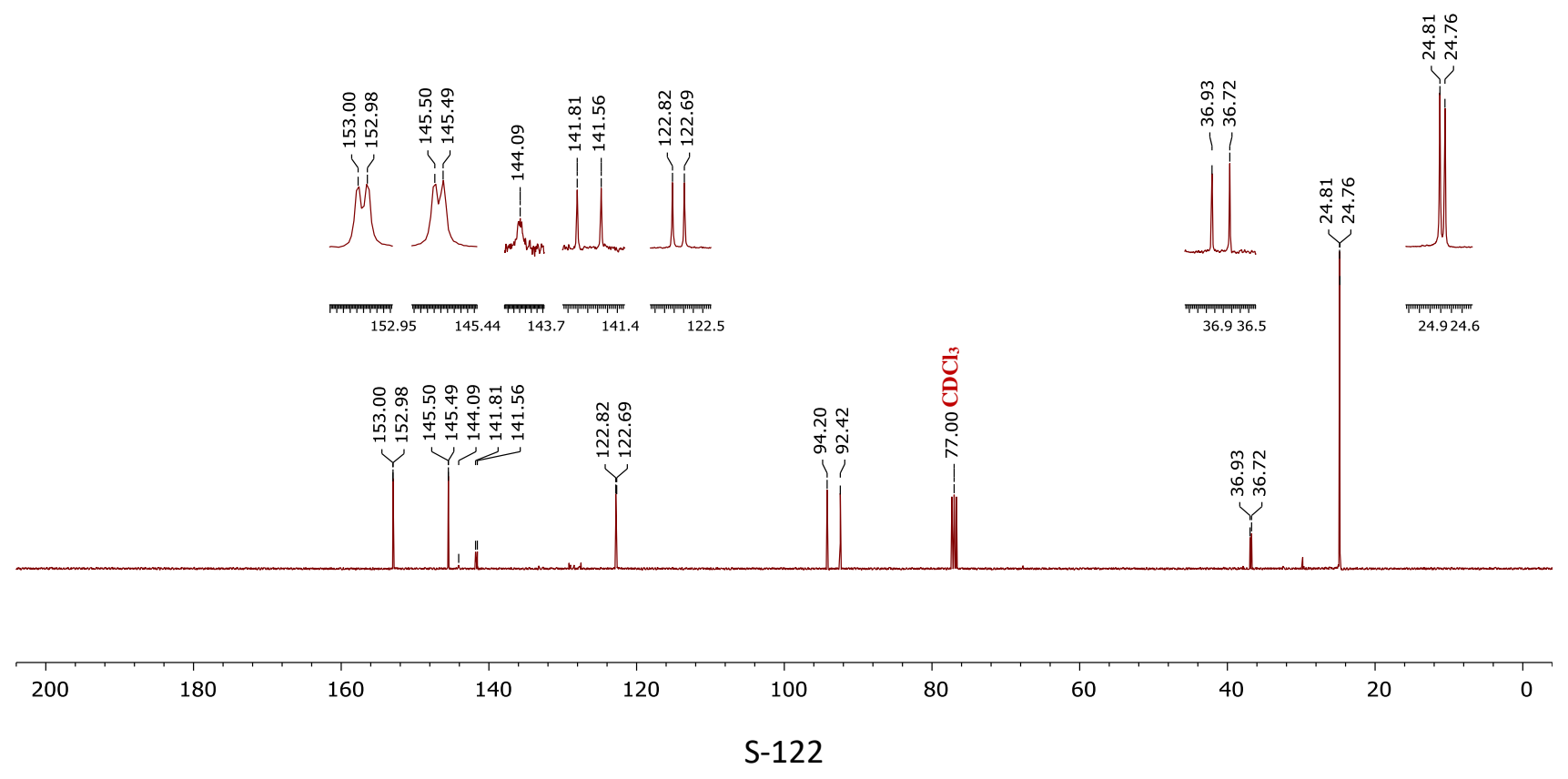


${ }^{19}$ F NMR (376 MHz, $\left.\mathrm{CDCl}_{3}\right)$

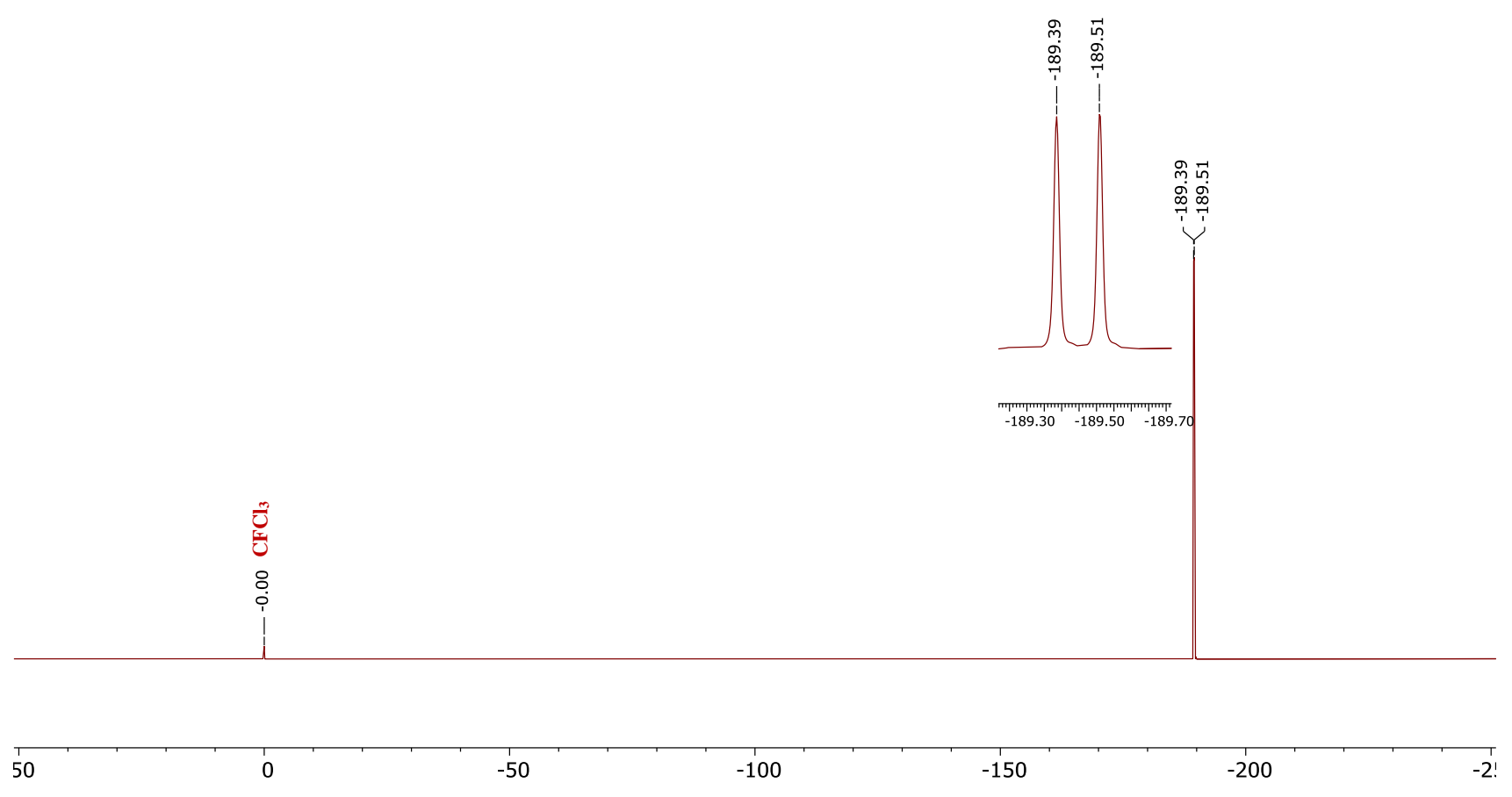


<smiles>CC(C)(C)C(F)c1cc([SbH2]c2ccccc2)ncc1[N+](=O)[O-]</smiles>
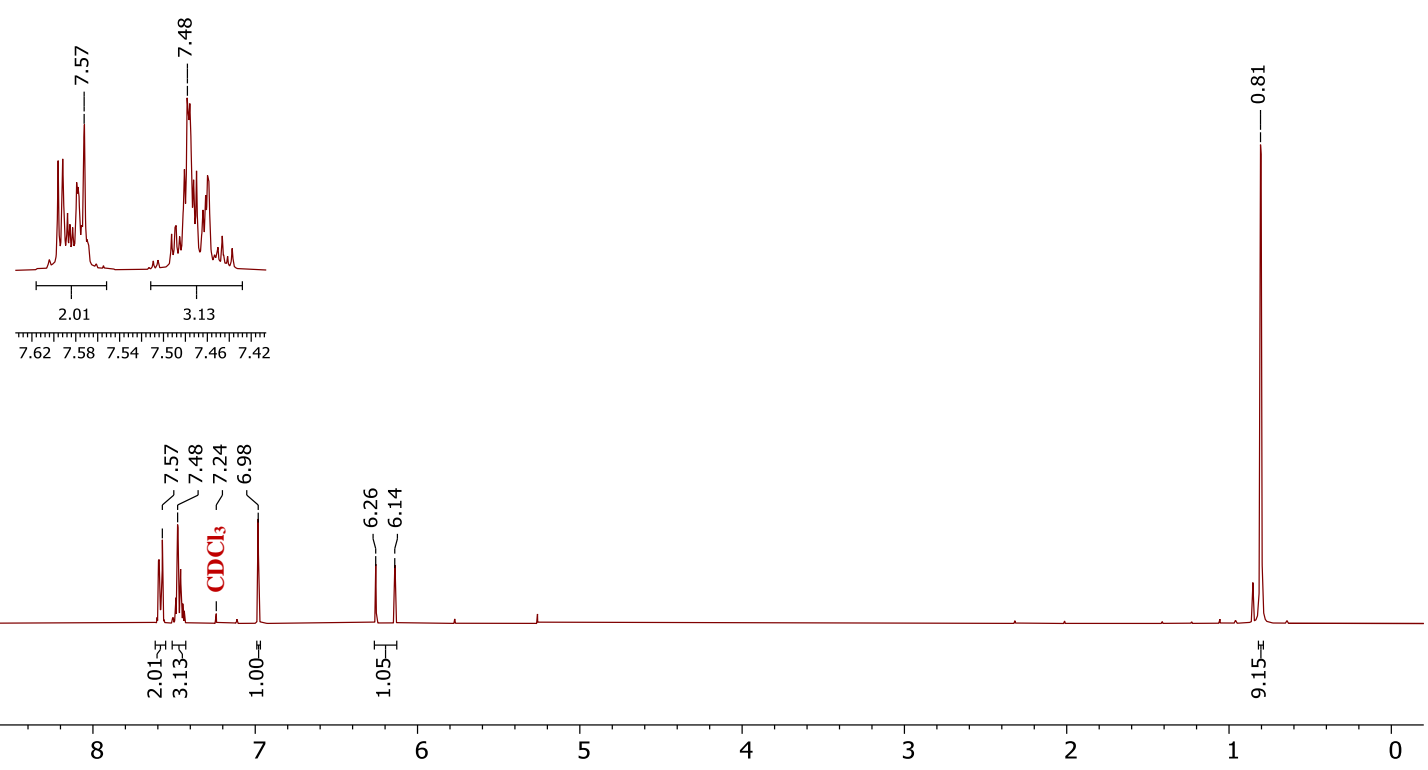

${ }^{13} \mathrm{C}$ NMR (100 MHz, $\left.\mathrm{CDCl}_{3}\right)$
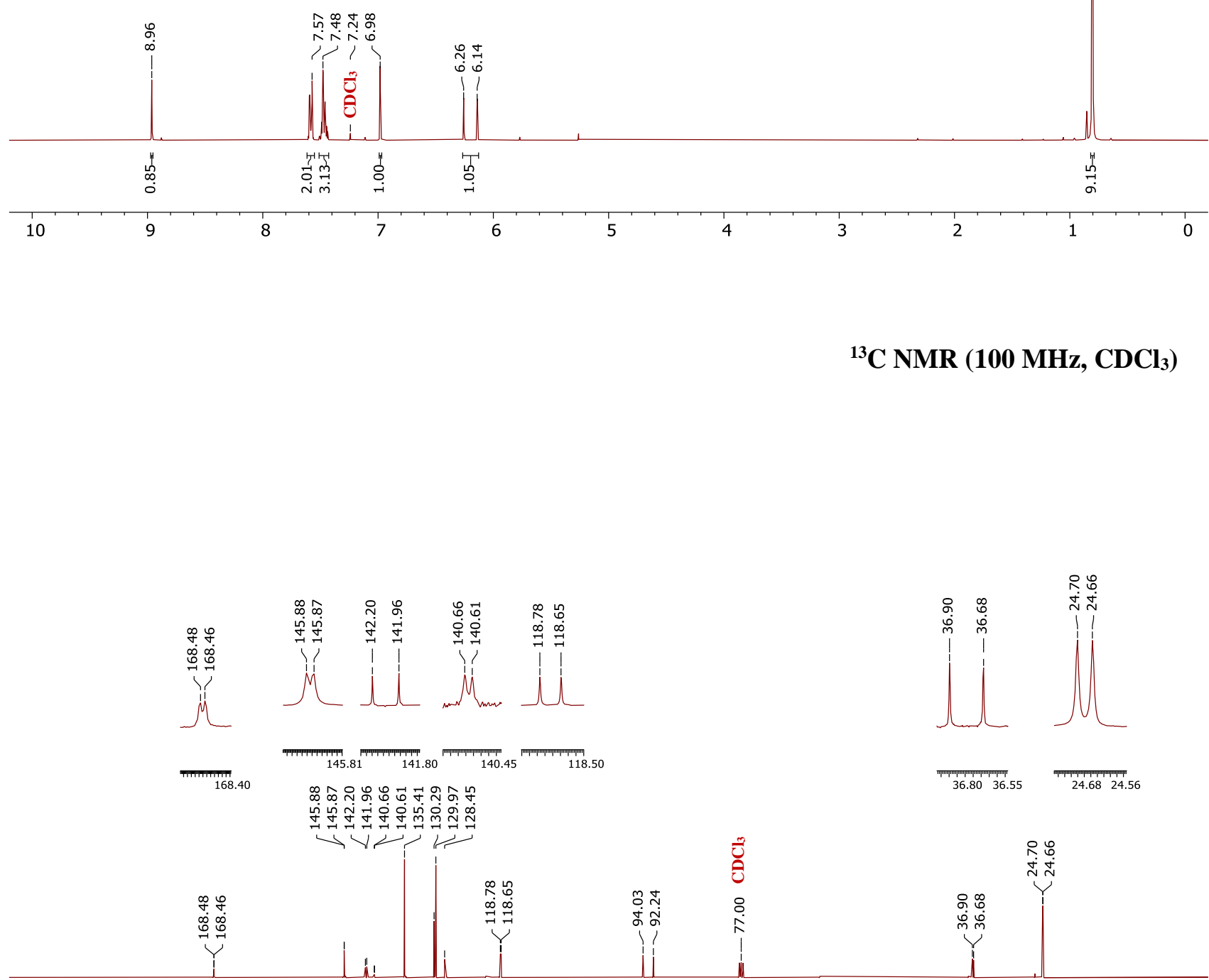
${ }^{19}$ F NMR (376 MHz, $\left.\mathrm{CDCl}_{3}\right)$
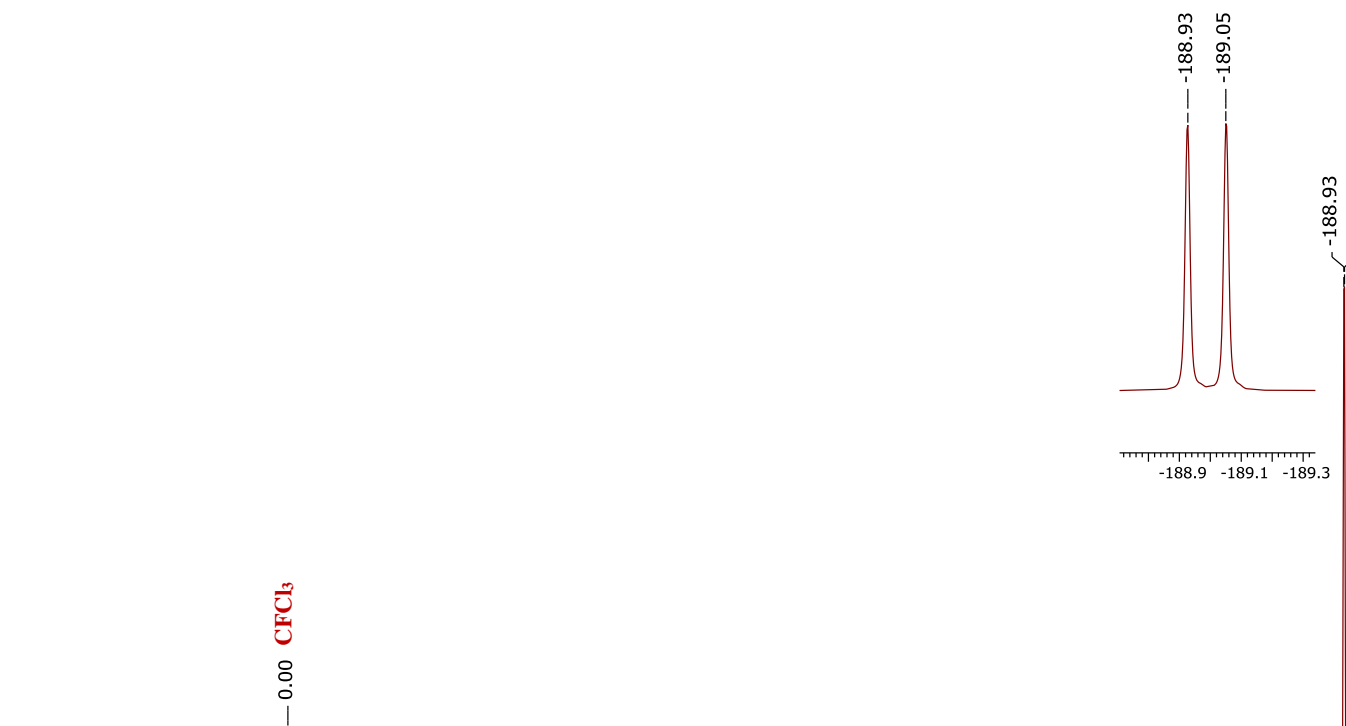

룬 
<smiles>CC(=O)Nc1cnccc1CC(C)C</smiles>

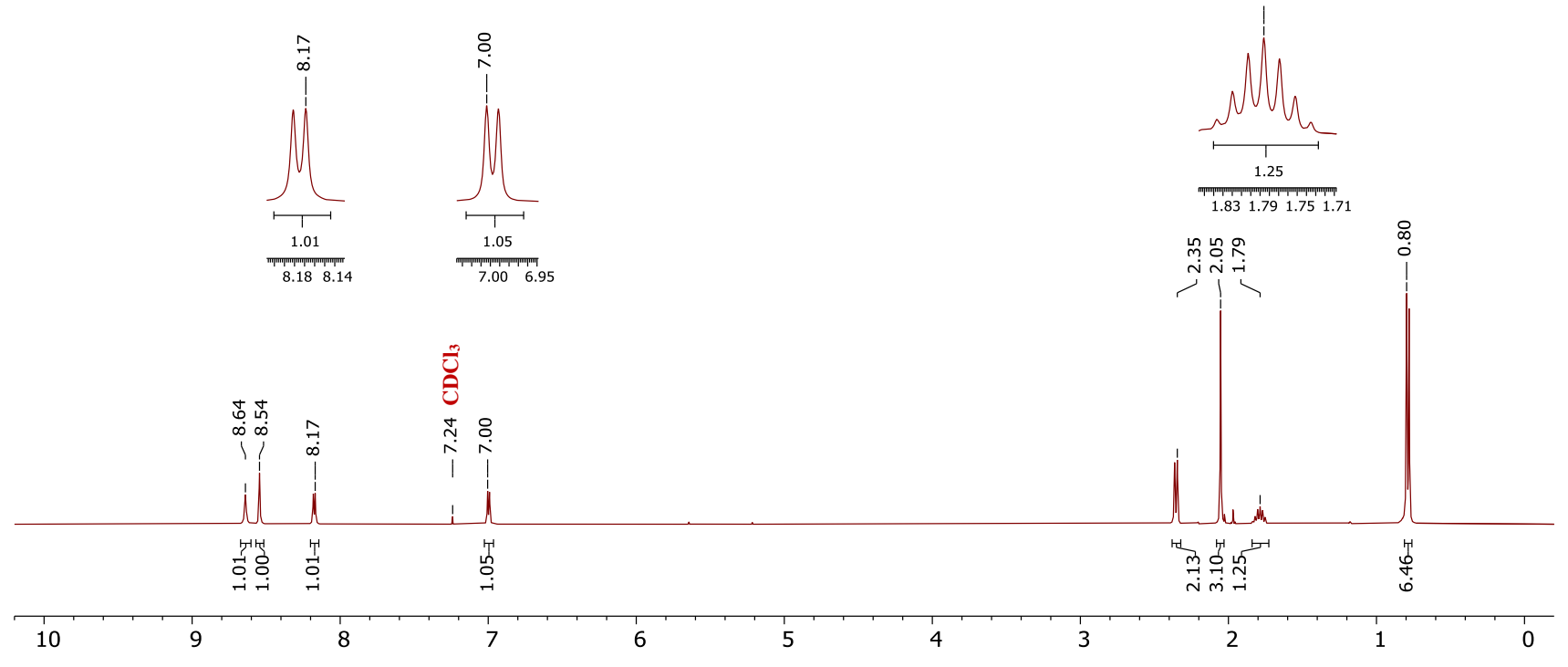

${ }^{13} \mathrm{C}$ NMR (100 MHz, $\left.\mathrm{CDCl}_{3}\right)$

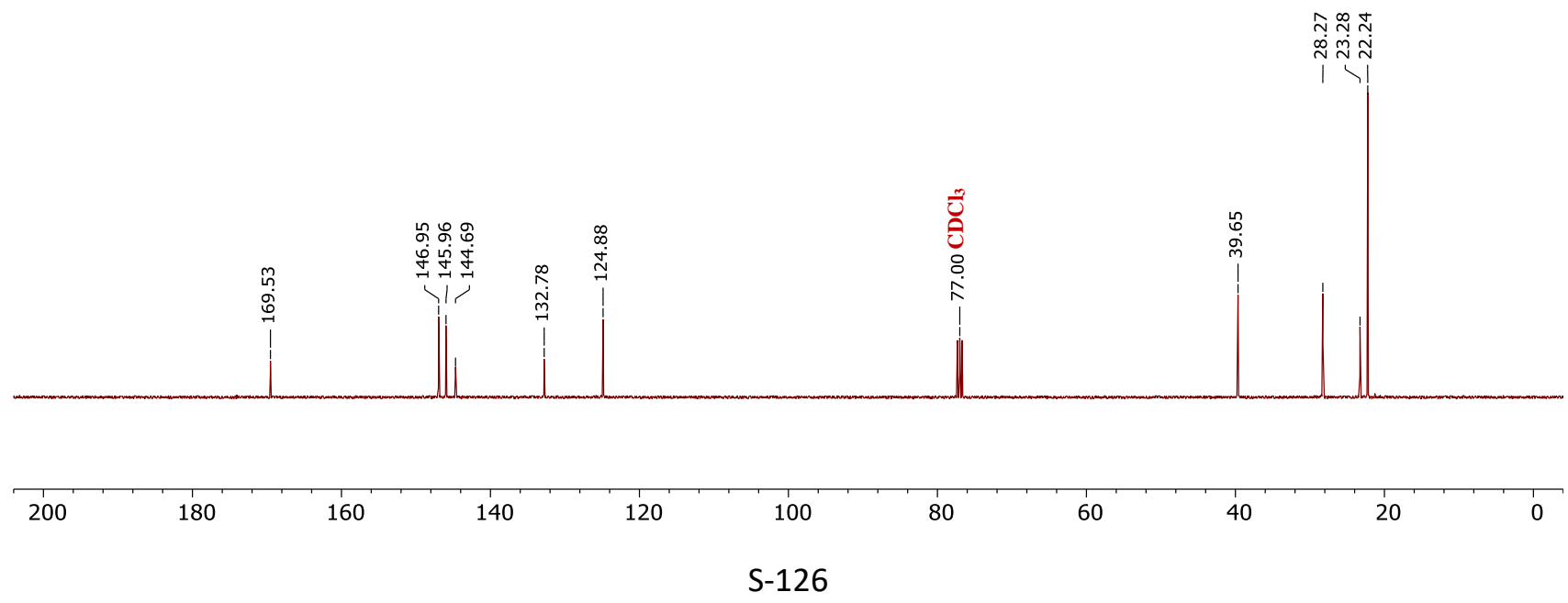


<smiles>CCCCCCCCc1cc(Cl)nc2cc[nH]c12</smiles>

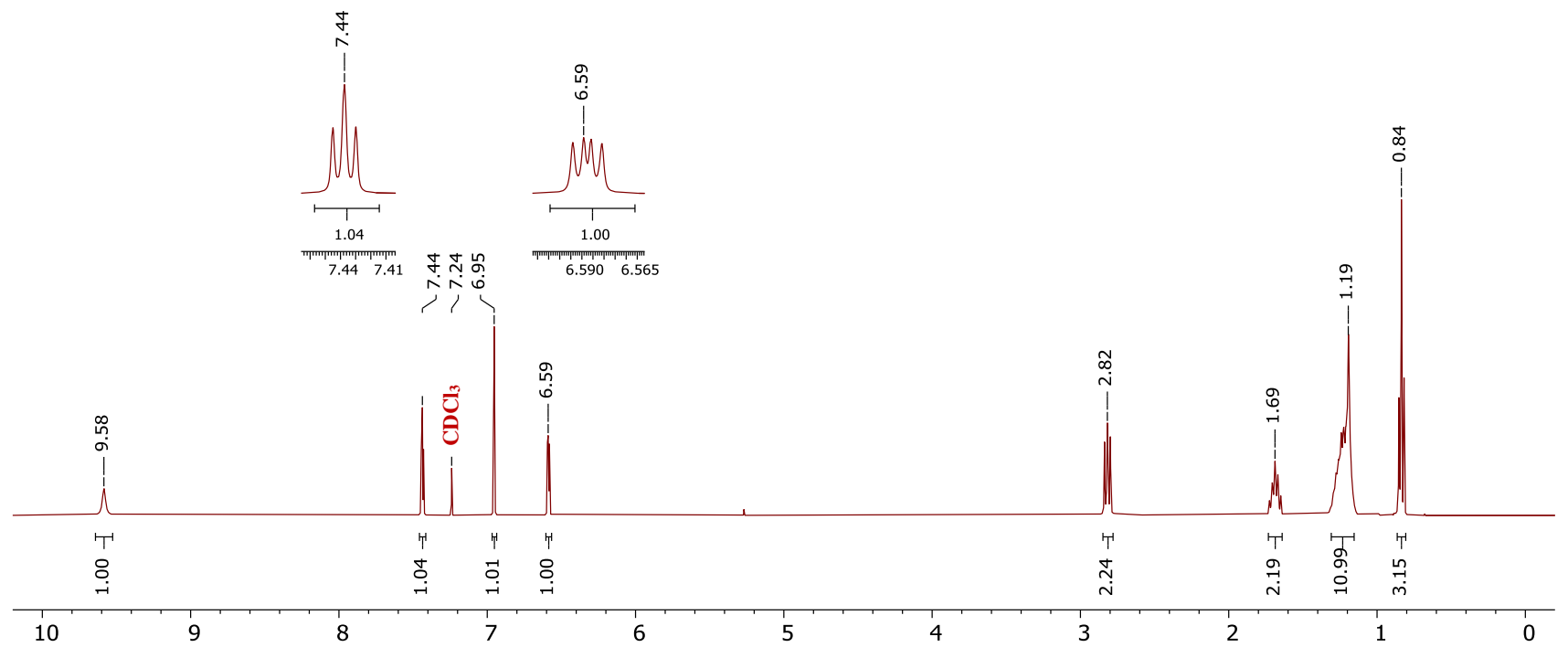

${ }^{13} \mathrm{C}$ NMR (100 MHz, $\left.\mathrm{CDCl}_{3}\right)$

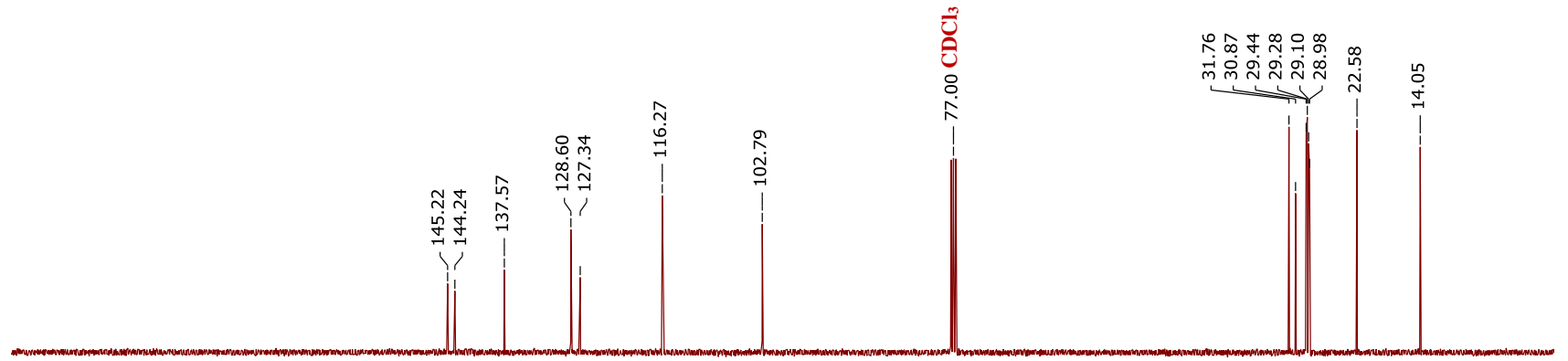

